\title{
Asymmetric Retro-Claisen Reaction by Chiral Primary Amine Catalysis
}

\author{
Yunbo Zhu, Long Zhang, and Sanzhong Luo* \\ Beijing National Laboratory for Molecule Sciences (BNLMS), CAS Key Laboratory for Molecular \\ Recognition and Function, Institute of Chemistry, Chinese Academy of Sciences, Beijing, 10019o, \\ China \\ Collaborative Innovation Center of Chemical Science and Engineering, Tianjin, 300o71, China
}

Supporting Information 


\section{Table of Contents}

General information and Materials...................................................S2

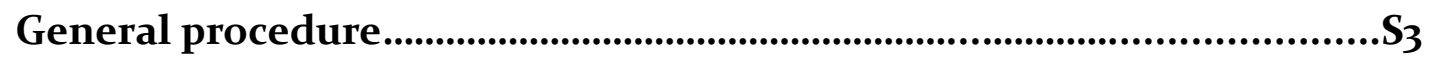

Mechanisitic studies................................................................................

Computational Studies................................................................S25

NMR Spectra..........................................................................................................

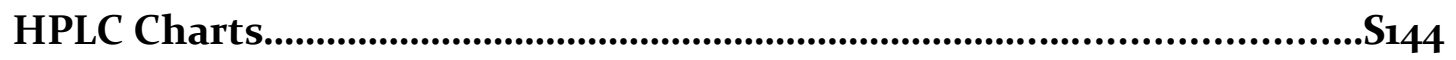

General information: Commercial reagents were purified prior to use following the guidelines of Perrin and Armarego. ${ }^{1}$ All solvents were purified according to the method of Grubbs. ${ }^{2}$ Nuclear magnetic resonance (NMR) spectra were recorded using Bruker AV-400 and AV-500 spectrometers. ${ }^{1} \mathrm{H}$ and ${ }^{13} \mathrm{CNMR}$ spectra were measured on a NMR instrument (400 and $500 \mathrm{MHz}$ for ${ }^{1} \mathrm{H}$ NMR, 100 and $125 \mathrm{MHz}$ for ${ }^{13} \mathrm{C}$ NMR). Tetramethylsilane (TMS) served as the internal standard for ${ }^{1} \mathrm{H} \mathrm{NMR}$, and $\mathrm{CDCl}_{3}$ and $\mathrm{CD}_{3} \mathrm{OD}$ served as the internal standard for ${ }^{13} \mathrm{C}$ NMR. The enantiomeric excesses were determined by HPLC analysis on Chiral Daicel Chiralpak AD-H, OD-H, AS-H, OJ-H columns. Optical rotation were measured on a commercial polarimeter and reported as follows: $[\alpha]_{\mathrm{D}}^{22}(\mathrm{c}=\mathrm{g} / 100 \mathrm{~mL}$, solvent). HRMS was recorded on a commercial instrument (ESI and APCI Source).

Materials: The corresponding ortho-quinone methide precursors 3 were prepared following known procedures ${ }^{3,4} \mathbf{2} \mathbf{b}$ and $\mathbf{2 c}$ was prepared according to literature precedent. ${ }^{5} \mathbf{2 a}, \mathbf{2 d - i}$ and $\mathbf{2 m}$ were prepared by alkylation of the corresponding $\alpha$-unsubstituted $\beta$-diketons with alkyl iodide or alkyl bromine. ${ }^{6}$ $\mathbf{2 j}$ was prepared according to literature procedure. ${ }^{7} \mathbf{2} \mathbf{k}$ was prepared according to literature procedure. ${ }^{8} \mathbf{2 l}$ was prepared according to literature procedure. ${ }^{9}$ Cyclic $\beta$-diketons 2n-p were prepared according to literature precedent. ${ }^{10}$ 


\section{General Procedure of the catalytic reactions}

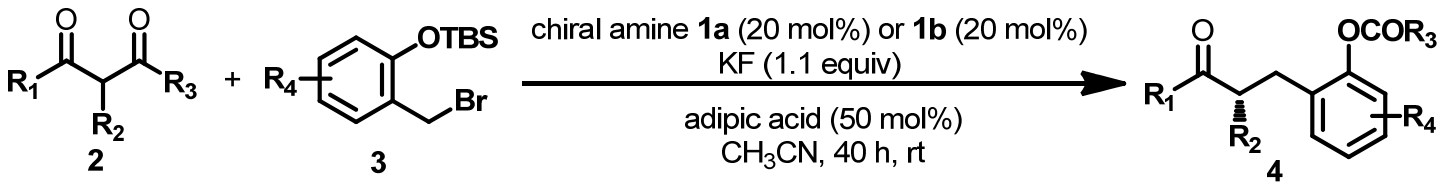

General procedure: To a mixture of 1,3-diketons 2 (o.1 mmol), chiral amine 1 (20 mol\%), adipic acid (50 mol\%) and KF (o.11 mmol) in a standard glass tube were added the corresponding bromide 3 ( $0.11 \mathrm{mmol})$, followed by $0.7 \mathrm{~mL}$ dry $\mathrm{MeCN}$ via syringe. The resultant mixture was stirred at room temperature until completion as indicated by TLC (<40 h). Solvent was removed and purification by silica gel column to give the desired product.

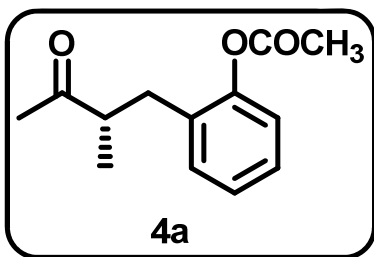

4a: Prepared according to the general procedure and obtained as colorless oil after purification by flash chromatography (20\% $\mathrm{Et}_{2} \mathrm{O} /$ petroleum ether). $86 \%$ yield, $95 \%$ ee. $[\alpha]_{\mathrm{D}}^{22}=+22.4\left(\mathrm{c}=1.0, \mathrm{CHCl}_{3}\right)$. HPLC analysis: Daicel Chiralpak AS-H, $2 \%$ iso-propanol/hexane, flow rate $=1.0 \mathrm{~mL} / \mathrm{min}, \lambda=205 \mathrm{~nm}$, retention time: $14.26 \min$ (major), $19.48 \mathrm{~min}$ (minor). ${ }^{1} \mathrm{H}$ NMR (40o $\left.\mathrm{MHz}, \mathrm{CDCl}_{3}\right) \delta 7.26-$ $7.13(\mathrm{~m}, 3 \mathrm{H}), 7.04(\mathrm{~d}, \mathrm{~J}=8.0 \mathrm{~Hz}, 1 \mathrm{H}), 2.94(\mathrm{dd}, \mathrm{J}=13.7,6.7 \mathrm{~Hz}, 1 \mathrm{H}), 2.86-2.73(\mathrm{~m}$ $1 \mathrm{H}), 2.48(\mathrm{dd}, \mathrm{J}=13.7,7.6 \mathrm{~Hz}, 1 \mathrm{H}), 2.33(\mathrm{~s}, 3 \mathrm{H}), 2.09(\mathrm{~s}, 3 \mathrm{H}), 1.09(\mathrm{~d}, \mathrm{~J}=7.0 \mathrm{~Hz}$, $3 \mathrm{H}) .{ }^{13} \mathrm{C}$ NMR $\left(100 \mathrm{MHz}, \mathrm{CDCl}_{3}\right) \delta 211.9,169.5,149.2,131.7,131.1,127.7,126.2,122.7$, 47.5, 33.3, 29.0, 21.1, 16.6. IR (thin film, $\mathrm{cm}^{-1}$ ) 2967, 2934, 1766, 1713, 1489, 1454, 1370, 1208, 1173, 1115, 1012, 916, 802, 749. HRMS (ESI) calcd for $\mathrm{C}_{13} \mathrm{H}_{16} \mathrm{O}_{3} \mathrm{Na}^{+}$: 243.0992, found 243.0993 .

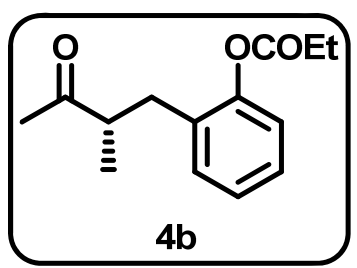

4b: Prepared according to the general procedure and obtained as colorless oil after purification by flash chromatography $\left(20 \% \mathrm{Et}_{2} \mathrm{O} /\right.$ petroleum ether). $74 \%$ 
yield, $95 \%$ ee. $[\alpha]_{\mathrm{D}}^{22}=+25.6\left(\mathrm{c}=1.0, \mathrm{CHCl}_{3}\right)$. HPLC analysis: Daicel Chiralpak AS- $\mathrm{H}, 5 \%$ iso-propanol $/$ hexane, flow rate $=1.0 \mathrm{~mL} / \mathrm{min}, \lambda=205 \mathrm{~nm}$, retention time: $7.58 \mathrm{~min}$ (major), $9.16 \mathrm{~min}$ (minor). ${ }^{1} \mathrm{H} \mathrm{NMR}\left(400 \mathrm{MHz}, \mathrm{CDCl}_{3}\right) \delta 7.26-$ $7.12(\mathrm{~m}, 3 \mathrm{H}), 7.05-7.01(\mathrm{~m}, 1 \mathrm{H}), 2.92(\mathrm{dd}, \mathrm{J}=13.6,6.7 \mathrm{~Hz}, 1 \mathrm{H}), 2.85-2.74(\mathrm{~m}, 1 \mathrm{H})$, $2.62(\mathrm{q}, \mathrm{J}=7.6 \mathrm{~Hz}, 2 \mathrm{H}), 2.47(\mathrm{dd}, \mathrm{J}=13.6,7.6 \mathrm{~Hz}, 1 \mathrm{H}), 2.08(\mathrm{~s}, 3 \mathrm{H}), 1.29(\mathrm{t}, \mathrm{J}=7.6$ $\mathrm{Hz}, 3 \mathrm{H}), 1.08(\mathrm{~d}, \mathrm{~J}=7.0 \mathrm{~Hz}, 3 \mathrm{H}) .{ }^{13} \mathrm{C} \mathrm{NMR}\left(100 \mathrm{MHz}, \mathrm{CDCl}_{3}\right) \delta$ 211.9, 173.0, 149.3, $131.7,131.1,127.7,126.1,122.7,47.5,33.4,29.0,27.9,16.6,9.3$. IR (thin film, $\mathrm{cm}^{-1}$ ) 2974, 2935, 1760, 1713, 1490, 1453, 1358, 1263, 1218, 1172, 1143, 1115, 803, 752. HRMS (ESI) calcd for $\mathrm{C}_{14} \mathrm{H}_{18} \mathrm{O}_{3} \mathrm{Na}^{+}$: 257.1148, found 257.1147.

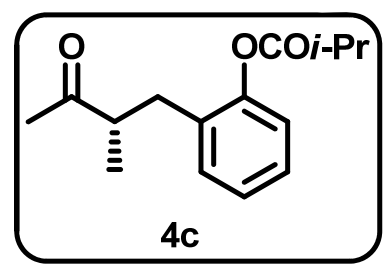

4c: Prepared according to the general procedure and obtained as colorless oil after purification by flash chromatography ( $25 \% \mathrm{Et}_{2} \mathrm{O} /$ petroleum ether). $55 \%$ yield, $96 \%$ ee. $[\alpha]_{\mathrm{D}}^{22}=21.2\left(\mathrm{c}=+1.0, \mathrm{CHCl}_{3}\right)$. HPLC analysis: Daicel Chiralpak $\mathrm{OJ}-\mathrm{H}, 3 \%$ iso-propanol $/$ hexane, flow rate $=1.0 \mathrm{~mL} / \mathrm{min}, \lambda=205 \mathrm{~nm}$, retention time: 8.94 min (major), 9.89 min (minor). ${ }^{1} \mathrm{H}$ NMR (40o $\left.\mathrm{MHz}, \mathrm{CDCl}_{3}\right) \delta 7.25-$ $7.12(\mathrm{~m}, 3 \mathrm{H}), 7.00(\mathrm{~d}, \mathrm{~J}=7.9 \mathrm{~Hz}, 1 \mathrm{H}), 2.93-2.77\left(\mathrm{~m},{ }_{3} \mathrm{H}\right), 2.49(\mathrm{dd}, \mathrm{J}=13.5,7.5 \mathrm{~Hz}$, $1 \mathrm{H}), 2.07(\mathrm{~s}, 3 \mathrm{H}), 1.34$ (d, J = 7.0 Hz, 6H), $1.08(\mathrm{~d}, \mathrm{~J}=7.0 \mathrm{~Hz}, 3 \mathrm{H}) .{ }^{13} \mathrm{C}$ NMR (100 $\left.\mathrm{MHz}, \mathrm{CDCl}_{3}\right) \delta$ 212.0, 175.6, 149.3, 131.8, 131.2, 127.7, 126.0, 122.6, 47.4, 34.4, 33.3, 29.1, 19.2, 16.6. IR (thin film, $\mathrm{cm}^{-1}$ ) 2976, 2937, 1755, 1715, 1489, 1456, 1363, 1218, 1172, 1116, 1096, 1047, 802, 752. HRMS (ESI) calcd for $\mathrm{C}_{15} \mathrm{H}_{20} \mathrm{O}_{3} \mathrm{Na}^{+}: 271.1305$, found 271.1305.

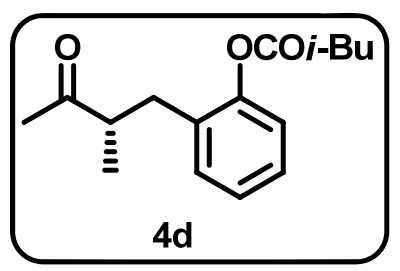

4d: Prepared according to the general procedure and obtained as colorless oil after purification by flash chromatography $\left(20 \% \mathrm{Et}_{2} \mathrm{O} /\right.$ petroleum ether). $70 \%$ yield, $95 \%$ ee. $[\alpha]_{\mathrm{D}}^{22}=+20.2\left(\mathrm{c}=1.01, \mathrm{CHCl}_{3}\right)$. HPLC analysis: Daicel Chiralpak 
AS-H, $5 \%$ iso-propanol/hexane, flow rate $=1.0 \mathrm{~mL} / \mathrm{min}, \lambda=205 \mathrm{~nm}$, retention time: $5.57 \mathrm{~min}$ (major), $6.13 \mathrm{~min}$ (minor). ${ }^{1} \mathrm{H}$ NMR (40o $\left.\mathrm{MHz}, \mathrm{CDCl}_{3}\right) \delta 7.25-$ $7.13(\mathrm{~m}, 3 \mathrm{H}), 7.02(\mathrm{~d}, \mathrm{~J}=7.8 \mathrm{~Hz}, 1 \mathrm{H}), 2.92(\mathrm{dd}, \mathrm{J}=13.6,6.8 \mathrm{~Hz}, 1 \mathrm{H}), 2.86-2.75(\mathrm{~m}$, $\left.{ }_{1} \mathrm{H}\right), 2.52-2.46(\mathrm{~m}, 3 \mathrm{H}), 2.26\left(\mathrm{dd}, \mathrm{J}=13.6,6.8 \mathrm{~Hz},{ }_{1} \mathrm{H}\right), 2.08(\mathrm{~s}, 3 \mathrm{H}), 1.08(\mathrm{~d}, \mathrm{~J}=$ $6.6 \mathrm{~Hz}, 9 \mathrm{H}) .{ }^{13} \mathrm{C}$ NMR (100 $\left.\mathrm{MHz}, \mathrm{CDCl}_{3}\right) \delta 211.9,171.6,149.3,131.7,131.2,127.7$, 126.1, 122.7, 47.4, 43.4, 33.4, 29.0, 25.9, 22.6, 16.6. IR (thin film, $\mathrm{cm}^{-1}$ ) 2963, 2933, 2876, 1758, 1715, 1490, 1454, 1362, 1292, 1218, 1172, 1152, 1114, 1101, 802, 750. HRMS (ESI) calcd for $\mathrm{C}_{16} \mathrm{H}_{22} \mathrm{O}_{3} \mathrm{Na}^{+}$: 285.1461, found 285.1463 .

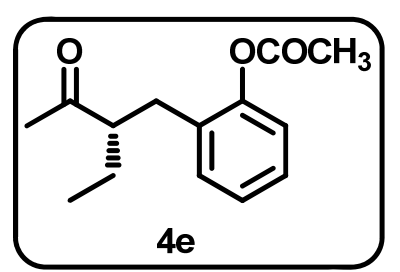

4e: Prepared according to the general procedure and obtained as colorless oil after purification by flash chromatography (15\% Et ${ }_{2} \mathrm{O} /$ petroleum ether). $79 \%$ yield, $97 \%$ ee. $[\alpha]_{\mathrm{D}}^{22}=+29.6\left(\mathrm{c}=1.0, \mathrm{CHCl}_{3}\right)$. HPLC analysis: Daicel Chiralpak AS-H, $5 \%$ iso-propanol/hexane, flow rate $=1.0 \mathrm{~mL} / \mathrm{min}, \lambda=205 \mathrm{~nm}$, retention time: $7.56 \mathrm{~min}$ (major), $8.54 \mathrm{~min}$ (minor). ${ }^{1} \mathrm{H}$ NMR (40o $\mathrm{MHz}, \mathrm{CDCl}_{3}$ ) $\delta 7.26-$ $7.12(\mathrm{~m}, 3 \mathrm{H}), 7.03(\mathrm{dd}, \mathrm{J}=8.0,0.8 \mathrm{~Hz}, 1 \mathrm{H}), 2.81(\mathrm{dd}, \mathrm{J}=13.2,8.1 \mathrm{~Hz}, 1 \mathrm{H}), 2.75-$ $2.69(\mathrm{~m}, 1 \mathrm{H}), 2.60(\mathrm{dd}, \mathrm{J}=13.2,5.7 \mathrm{~Hz}, 1 \mathrm{H}), 2.33\left(\mathrm{~s},{ }_{3} \mathrm{H}\right), 2.01(\mathrm{~s}, 3 \mathrm{H}), 1.70-1.58$ $(\mathrm{m}, 1 \mathrm{H}), 1.56-1.44(\mathrm{~m}, 1 \mathrm{H}), 0.90(\mathrm{t}, \mathrm{J}=7.4 \mathrm{~Hz}, 3 \mathrm{H}) .{ }^{13} \mathrm{C} \mathrm{NMR}\left(100 \mathrm{MHz}, \mathrm{CDCl}_{3}\right) \delta$ 212.1, 169.6, 149.1, 131.9, 131.1, 127.7, 126.3, 122.7, 54.7, 31.8, 30.4, 24.9, 21.1, 11.8. IR (thin film, $\mathrm{cm}^{-1}$ ) 2964, 2934, 1766, 1712, 1489, 1454, 1369, 1210, 1172, 1117, 1103, 1012, 916, 8oo, 752. HRMS (ESI) calcd for $\mathrm{C}_{14} \mathrm{H}_{18} \mathrm{O}_{3} \mathrm{Na}^{+}$: 257.1148, found 257.1148.

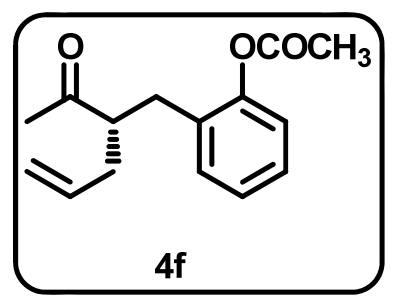

4f: Prepared according to the general procedure and obtained as colorless oil after purification by flash chromatography (20\% $\mathrm{Et}_{2} \mathrm{O} /$ petroleum ether). $84 \%$ yield, $96 \%$ ee. $[\alpha]_{\mathrm{D}}{ }^{22}=+12.0\left(\mathrm{c}=1.0, \mathrm{CHCl}_{3}\right)$. HPLC analysis: Daicel Chiralpak 
AS-H, $5 \%$ iso-propanol/hexane, flow rate $=1.0 \mathrm{~mL} / \mathrm{min}, \lambda=205 \mathrm{~nm}$, retention time: $7.75 \mathrm{~min}$ (major), $8.47 \mathrm{~min}$ (minor). ${ }^{1} \mathrm{H}$ NMR (400 $\left.\mathrm{MHz}, \mathrm{CDCl}_{3}\right) \delta 7.25-$ $7.11\left(\mathrm{~m},{ }_{3} \mathrm{H}\right), 7.03\left(\mathrm{~d}, \mathrm{~J}=7.9 \mathrm{~Hz},{ }_{1} \mathrm{H}\right), 5.78-5.67\left(\mathrm{~m},{ }_{1} \mathrm{H}\right), 5.13-5.02(\mathrm{~m}, 2 \mathrm{H}), 2.90$ - $2.77(\mathrm{~m}, 2 \mathrm{H}), 2.68-2.60(\mathrm{~m}, 1 \mathrm{H}), 2.41-2.30(\mathrm{~m}, 4 \mathrm{H}), 2.23-2.12(\mathrm{~m}, 1 \mathrm{H}), 1.99(\mathrm{~s}$, $3 \mathrm{H}) .{ }^{13} \mathrm{C} \mathrm{NMR}\left(100 \mathrm{MHz}, \mathrm{CDCl}_{3}\right) \delta 211.3,169.5,149.1,135.2,131.6,131.2,127.8,126.3$, 122.7, 117.6, 52.8, 36.1, 31.8, 30.7, 21.1. IR (thin film, $\mathrm{cm}^{-1}$ ) 2962, 2926, 1766, 1713, 1489, 1369, 1209, 1173, 1098, 1012, 915, 800, 750. HRMS (ESI) calcd for $\mathrm{C}_{15} \mathrm{H}_{18} \mathrm{O}_{3} \mathrm{Na}^{+}$: 269.1148, found 269.1150 .

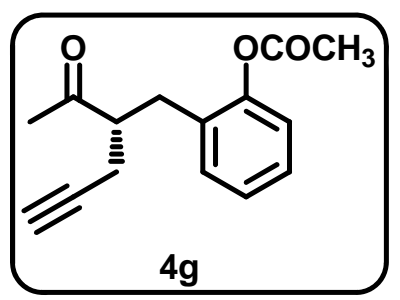

4g: Prepared according to the general procedure and obtained as colorless oil after purification by flash chromatography (15\% $\mathrm{Et}_{2} \mathrm{O} /$ petroleum ether). $80 \%$ yield, $95 \%$ ee. $[\alpha]_{\mathrm{D}}{ }^{22}=-9.1\left(\mathrm{c}=1.0, \mathrm{CHCl}_{3}\right)$. HPLC analysis: Daicel Chiralpak AS-H, $5 \%$ iso-propanol/hexane, flow rate $=1.0 \mathrm{~mL} / \mathrm{min}, \lambda=205 \mathrm{~nm}$, retention time: $12.75 \mathrm{~min}$ (major), $14.67 \mathrm{~min}$ (minor). ${ }^{1} \mathrm{H} \mathrm{NMR}\left(400 \mathrm{MHz}, \mathrm{CDCl}_{3}\right) \delta_{7.29}-$ $7.23(\mathrm{~m}, 1 \mathrm{H}), 7.23-7.14(\mathrm{~m}, 2 \mathrm{H}), 7.05(\mathrm{dd}, \mathrm{J}=8.0,0.8 \mathrm{~Hz}, 1 \mathrm{H}), 3.00-2.90(\mathrm{~m}$, $\left.{ }_{1 H}\right), 2.83(\mathrm{dd}, \mathrm{J}=7.3,1.8 \mathrm{~Hz}, 2 \mathrm{H}), 2.38(\mathrm{td}, \mathrm{J}=6.7,2.6 \mathrm{~Hz}, 2 \mathrm{H}), 2.34(\mathrm{~s}, 3 \mathrm{H}), 2.06$ $(\mathrm{s}, 3 \mathrm{H}), 2.03(\mathrm{t}, \mathrm{J}=2.6 \mathrm{~Hz}, 1 \mathrm{H}) .{ }^{13} \mathrm{C} \mathrm{NMR}\left(100 \mathrm{MHz}, \mathrm{CDCl}_{3}\right) \delta$ 209.9, 169.6, 149.2, $131.2,130.9,128.1,126.4,122.9,81.5,70.5,51.6,31.6,30.7,21.1,20.6$. IR (thin film, $\mathrm{cm}^{-1}$ ) 3286, 2962, 2929, 1761, 1714, 1490, 1452, 1428, 1369, 1208, 1174, 1101, 1012, 914, 797, 751, 661. HRMS (ESI) calcd for $\mathrm{C}_{15} \mathrm{H}_{16} \mathrm{O}_{3} \mathrm{Na}^{+}$: 267.0992, found 2467.0993.

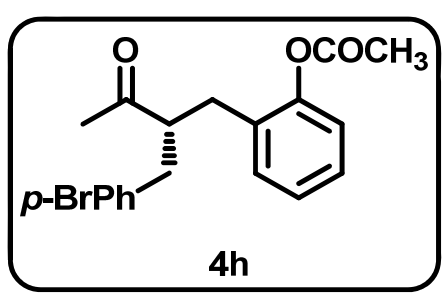

4h: Prepared according to the general procedure and obtained as colorless oil after purification by flash chromatography (25\% Et ${ }_{2} \mathrm{O} /$ petroleum ether). $40 \%$ 
yield, $97 \%$ ee. $[\alpha]_{\mathrm{D}}{ }^{22}=-5.6\left(\mathrm{c}=1.0, \mathrm{CHCl}_{3}\right)$. HPLC analysis: Daicel Chiralpak $\mathrm{OJ}-\mathrm{H}, 20 \%$ iso-propanol $/$ hexane, flow rate $=1.0 \mathrm{~mL} / \mathrm{min}, \lambda=205 \mathrm{~nm}$, retention time: $16.97 \mathrm{~min}$ (major), $13.05 \mathrm{~min}$ (minor). ${ }^{1} \mathrm{H}$ NMR (400 MHz, $\left.\mathrm{CDCl}_{3}\right) \delta 7.40(\mathrm{~d}$, $\mathrm{J}=8.3 \mathrm{~Hz}, 2 \mathrm{H}), 7.25-7.21(\mathrm{~m}, 1 \mathrm{H}), 7.18-7.13(\mathrm{~m}, 2 \mathrm{H}), 7.02\left(\mathrm{~d}, \mathrm{~J}=8.3 \mathrm{~Hz},{ }_{3} \mathrm{H}\right)$, $3.08-3.01(\mathrm{~m}, 1 \mathrm{H}), 2.89-2.79(\mathrm{~m}, 2 \mathrm{H}), 2.64-2.58(\mathrm{~m}, 2 \mathrm{H}), 2.19(\mathrm{~s}, 3 \mathrm{H}), 1.83(\mathrm{~s}$, $3 \mathrm{H}) .{ }^{13} \mathrm{C}$ NMR (10o MHz, $\mathrm{CDCl}_{3}$ ) $\delta$ 211.6, 169.6, 149.1, 138.4, 131.8, 131.2, 131.2, 130.9, 128.0, 126.4, 122.8, 120.5, 54.8, 37.6, 32.5, 31.7, 20.9. IR (thin film, $\mathrm{cm}^{-1}$ ) 2962, 2926, 1764, 1713, 1488, 1449, 1368, 1261, 1208, 1172, 1096, 1071, 1011, 915, 798, 753. HRMS (ESI) calcd for $\mathrm{C}_{19} \mathrm{H}_{19} \mathrm{O}_{3} \mathrm{BrNa}^{+}$: 397.0410, found 397.0409.

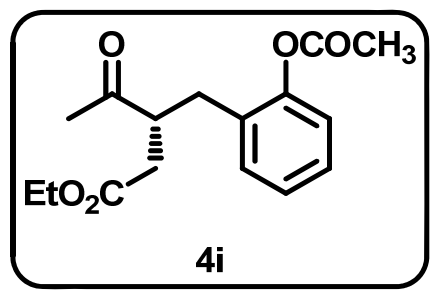

4i: Prepared according to the general procedure and obtained as colorless oil after purification by flash chromatography $\left(30 \% \mathrm{Et}_{2} \mathrm{O}\right.$ /petroleum ether). $68 \%$ yield, $92 \%$ ee. $[\alpha]_{\mathrm{D}}^{22}=+69.7\left(\mathrm{c}=1.01, \mathrm{CHCl}_{3}\right)$. HPLC analysis: Daicel Chiralpak OD-H, $5 \%$ iso-propanol/hexane, flow rate $=1.0 \mathrm{~mL} / \mathrm{min}, \lambda=205 \mathrm{~nm}$, retention time: $16.68 \mathrm{~min}$ (major), $14.04 \mathrm{~min}$ (minor). ${ }^{1} \mathrm{H}$ NMR (40o MHz, $\left.\mathrm{CDCl}_{3}\right) \delta 7.29-$ $7.25(\mathrm{~m}, 1 \mathrm{H}), 7.22-7.15(\mathrm{~m}, 2 \mathrm{H}), 7.07(\mathrm{~d}, \mathrm{~J}=8.0 \mathrm{~Hz}, 1 \mathrm{H}), 4.07(\mathrm{q}, \mathrm{J}=7.1 \mathrm{~Hz}, 2 \mathrm{H})$, $3.31-3.17(\mathrm{~m}, 1 \mathrm{H}), 2.83(\mathrm{dd}, \mathrm{J}=13.7,7.2 \mathrm{~Hz}, 1 \mathrm{H}), 2.73(\mathrm{dd}, \mathrm{J}=17.1,9.5 \mathrm{~Hz}, 1 \mathrm{H})$, $2.55(\mathrm{dd}, \mathrm{J}=13.7,8.0 \mathrm{~Hz}, 1 \mathrm{H}), 2.36(\mathrm{~s}, 3 \mathrm{H}), 2.31(\mathrm{dd}, \mathrm{J}=17.7,13.0 \mathrm{~Hz}, 1 \mathrm{H}), 2.11(\mathrm{~s}$, $3 \mathrm{H}), 1.22(\mathrm{t}, \mathrm{J}=7.1 \mathrm{~Hz}, 3 \mathrm{H}) .{ }^{13} \mathrm{C}$ NMR $\left(100 \mathrm{MHz}, \mathrm{CDCl}_{3}\right) \delta$ 210.6, 172.2, 169.5, 149.2, 131.1, 130.4, 128.2, 126.4, 122.9, 6o.8, 48.2, 35.6, 32.4, 30.5, 21.1, 14.2. IR (thin film, $\mathrm{cm}^{-1}$ ) 2968, 2929, 1766, 1732, 1716, 1491, 1370, 1207, 1173, 1100, 1014, 916, 800, 753. HRMS (ESI) calcd for $\mathrm{C}_{16} \mathrm{H}_{20} \mathrm{O}_{5} \mathrm{Na}^{+}: 315.1203$, found 315.1203 .

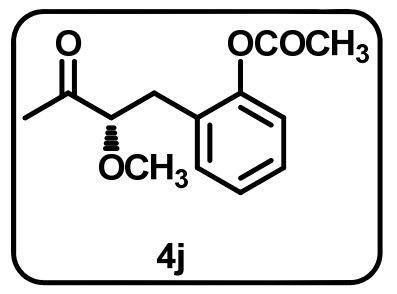

4j: Prepared according to the general procedure and obtained as colorless oil 
after purification by flash chromatography ( $20 \% \mathrm{Et}_{2} \mathrm{O} /$ petroleum ether). $60 \%$ yield, $96 \% e e .[\alpha]_{\mathrm{D}}^{22}=-36.0\left(\mathrm{c}=1.0, \mathrm{CHCl}_{3}\right)$. HPLC analysis: Daicel Chiralpak AS- $\mathrm{H}^{*} 2,3 \%$ iso-propanol/hexane, flow rate $=0.6 \mathrm{~mL} / \mathrm{min}, \lambda=207 \mathrm{~nm}$, retention time: 33.80 min (major), $35.04 \mathrm{~min}$ (minor). ${ }^{1} \mathrm{H}$ NMR $\left(500 \mathrm{MHz}, \mathrm{CDCl}_{3}\right) \delta 7.29$ $(\mathrm{td}, \mathrm{J}=7.4,1.6 \mathrm{~Hz}, 2 \mathrm{H}), 7.18(\mathrm{td}, \mathrm{J}=7.5,1.1 \mathrm{~Hz}, 1 \mathrm{H}), 7.09-7.04(\mathrm{~m}, 1 \mathrm{H}), 3.74(\mathrm{dd}$, $\mathrm{J}=8.3,4.5 \mathrm{~Hz}, 1 \mathrm{H}), 3.27(\mathrm{~s}, 3 \mathrm{H}), 2.89(\mathrm{dd}, \mathrm{J}=14.1,4.4 \mathrm{~Hz}, 1 \mathrm{H}), 2.8 \mathrm{o}(\mathrm{dd}, \mathrm{J}=14.1$, $8.3 \mathrm{~Hz}, 1 \mathrm{H}), 2.34(\mathrm{~s}, 3 \mathrm{H}), 2.14(\mathrm{~s}, 3 \mathrm{H}) .{ }^{13} \mathrm{C}$ NMR $\left(100 \mathrm{MHz}, \mathrm{CDCl}_{3}\right) \delta$ 210.9, 169.6, $149.3,131.7,129.7,128.1,126.2,122.7,87.4,58.8,33.1,25.8,21.1$. IR (thin film, $\mathrm{cm}^{-1}$ ) 2970, 2932, 1766, 1715, 1490, 1455, 1369, 126o, 1208, 1174, 1104, 1041, 1012, 916, 801, 752. HRMS (ESI) calcd for $\mathrm{C}_{13} \mathrm{H}_{16} \mathrm{O}_{4} \mathrm{Na}^{+}$: 259.0941, found 259.0941.

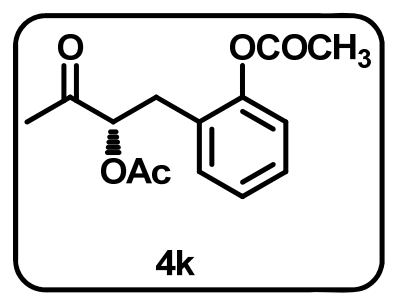

4k: Prepared according to the general procedure and obtained as colorless oil after purification by flash chromatography ( $50 \% \mathrm{Et}_{2} \mathrm{O} /$ petroleum ether). $98 \%$ yield, $94 \%$ ee. $[\alpha]_{D}^{22}=+4.0\left(\mathrm{c}=1.0, \mathrm{CHCl}_{3}\right)$. HPLC analysis: Daicel Chiralpak $\mathrm{OJ}-\mathrm{H}, 20 \%$ iso-propanol $/$ hexane, flow rate $=1.0 \mathrm{~mL} / \mathrm{min}, \lambda=206 \mathrm{~nm}$, retention time: $12.79 \mathrm{~min}$ (major), $14.71 \mathrm{~min}$ (minor). ${ }^{1} \mathrm{H}$ NMR (40o $\left.\mathrm{MHz}, \mathrm{CDCl}_{3}\right) \delta 7.31-$ $7.25(\mathrm{~m}, 2 \mathrm{H}), 7.22-7.15(\mathrm{~m}, 1 \mathrm{H}), 7.08(\mathrm{~d}, \mathrm{~J}=8.0 \mathrm{~Hz}, 1 \mathrm{H}), 5.18(\mathrm{dd}, \mathrm{J}=8.3,5.1 \mathrm{~Hz}$, 1H), $3.05(\mathrm{dd}, \mathrm{J}=14.3,5.1 \mathrm{~Hz}, 1 \mathrm{H}), 2.94(\mathrm{dd}, \mathrm{J}=14.3,8.3 \mathrm{~Hz}, 1 \mathrm{H}), 2.35(\mathrm{~s}, 3 \mathrm{H}), 2.10$ (s, $3 \mathrm{H}), 2.07(\mathrm{~s}, 3 \mathrm{H}) .{ }^{13} \mathrm{C}$ NMR (100 MHz, $\mathrm{CDCl}_{3}$ ) $\delta$ 205.3, 170.5, 169.4, 149.4, 131.4, 128.5, 128.1, 126.3, 122.8, 78.2, 31.3, 26.9, 21.1, 20.7. IR (thin film, $\mathrm{cm}^{-1}$ ) 2968, 2929, 1766, 1744, 1731, 1490, 1455, 1372, 1239, 1206, 1172, 1105, 1074, 1045, 1012, 914, 799, 752. HRMS (ESI) calcd for $\mathrm{C}_{14} \mathrm{H}_{16} \mathrm{O}_{5} \mathrm{Na}^{+}: 287.0890$, found 287.0891 .

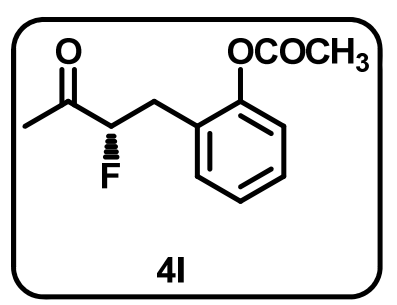

41: Prepared according to the general procedure and obtained as colorless oil 
after purification by flash chromatography ( $20 \% \mathrm{Et}_{2} \mathrm{O} /$ petroleum ether). $42 \%$ yield, $60 \%$ ee. $[\alpha]_{\mathrm{D}}{ }^{22}=-36.0\left(\mathrm{c}=0.3, \mathrm{CHCl}_{3}\right)$. HPLC analysis: Daicel Chiralpak AS- $\mathrm{H}, 5 \%$ iso-propanol/hexane, flow rate $=1.0 \mathrm{~mL} / \mathrm{min}, \lambda=205 \mathrm{~nm}$, retention time: $10.17 \mathrm{~min}$ (major), $9.27 \mathrm{~min}$ (minor). ${ }^{1} \mathrm{H} \mathrm{NMR}\left(400 \mathrm{MHz}, \mathrm{CDCl}_{3}\right) \delta 7.33-$ $7.31(\mathrm{~m}, 2 \mathrm{H}), 7.24-7.16(\mathrm{~m}, 1 \mathrm{H}), 7.09(\mathrm{~d}, \mathrm{~J}=8.1 \mathrm{~Hz}, 1 \mathrm{H}), 4.89$ (ddd, J = 49.9, 8.o, $3.6 \mathrm{~Hz}, 1 \mathrm{H}), 3.17(\mathrm{ddd}, \mathrm{J}=29.9,14.9,3.6 \mathrm{~Hz}, 1 \mathrm{H}), 2.95(\mathrm{ddd}, \mathrm{J}=23.1,14.9,8.0 \mathrm{~Hz}$, $1 \mathrm{H}), 2.34(\mathrm{~s}, 3 \mathrm{H}), 2.19\left(\mathrm{~d}, \mathrm{~J}=4.9 \mathrm{~Hz},{ }_{3} \mathrm{H}\right) .{ }^{13} \mathrm{C} \mathrm{NMR}\left(100 \mathrm{MHz}, \mathrm{CDCl}_{3}\right) \delta 208.3$, 207.7, 169.5, 149.3, 131.7, 128.6, 127.8, 126.3, 122.9, 96.2, 94.4, 32.9, 32.7, 26.4, 21.1. IR (thin film, $\mathrm{cm}^{-1}$ ) 2965, 2923, 176o, 1723, 1491, 1369, 1260, 1207, 1172, 1103, 1077, 1015, 915, 80o, 750. HRMS (ESI) calcd for $\mathrm{C}_{12} \mathrm{H}_{13} \mathrm{O}_{3} \mathrm{FNa}^{+}$: 247.0741, found 247.0742.

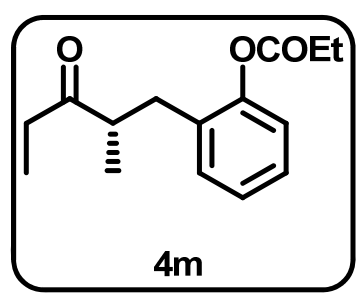

4m: Prepared according to the general procedure and obtained as colorless oil after purification by flash chromatography ( $20 \% \mathrm{Et}_{2} \mathrm{O} /$ petroleum ether). $30 \%$ yield, $96 \%$ ee. $[\alpha]_{\mathrm{D}}^{22}=+33.9\left(\mathrm{c}=0.66, \mathrm{CHCl}_{3}\right)$. HPLC analysis: Daicel Chiralpak AS- $\mathrm{H}, 5 \%$ iso-propanol $/$ hexane, flow rate $=1.0 \mathrm{~mL} / \mathrm{min}, \lambda=205 \mathrm{~nm}$, retention time: $5.43 \mathrm{~min}$ (major), $6.34 \mathrm{~min}$ (minor). ${ }^{1} \mathrm{H} \mathrm{NMR}\left(400 \mathrm{MHz}, \mathrm{CDCl}_{3}\right) \delta 7.25-$ $7.11(\mathrm{~m}, 3 \mathrm{H}), 7.02(\mathrm{~d}, \mathrm{~J}=8.0 \mathrm{~Hz}, 1 \mathrm{H}), 2.89(\mathrm{dd}, \mathrm{J}=13.3,7.1 \mathrm{~Hz}, 1 \mathrm{H}), 2.81(\mathrm{dd}, \mathrm{J}=$ 13.9, 7.o Hz, $1 \mathrm{H}), 2.62$ (q, J = 7.5 Hz, 2H), $2.54-2.36(\mathrm{~m}, 2 \mathrm{H}), 2.29-2.20(\mathrm{~m}, 1 \mathrm{H})$, $1.30\left(\mathrm{t}, \mathrm{J}=7.6 \mathrm{~Hz},{ }_{3} \mathrm{H}\right), 1.07\left(\mathrm{~d}, \mathrm{~J}=6.8 \mathrm{~Hz},{ }_{3} \mathrm{H}\right), 0.97\left(\mathrm{t}, \mathrm{J}=7.3 \mathrm{~Hz},{ }_{3} \mathrm{H}\right) .{ }^{13} \mathrm{C} \mathrm{NMR}$ $\left(100 \mathrm{MHz}, \mathrm{CDCl}_{3}\right) \delta 214.7,173.0,149.3,131.9,131.2,127.7,126.1,122.7,46.5,35.4$, 33.8, 27.9, 17.0, 9.4, 7.7. IR (thin film, $\mathrm{cm}^{-1}$ ) 2970, 2935, 1760, 1714, 1489, 1455, 1383, 1358, 1262, 1218, 1172, 1142, 1109, 1076, 983, 803, 753. HRMS (ESI) calcd for $\mathrm{C}_{15} \mathrm{H}_{20} \mathrm{O}_{3} \mathrm{Na}^{+}$: 271.1305, found 271.1305. 


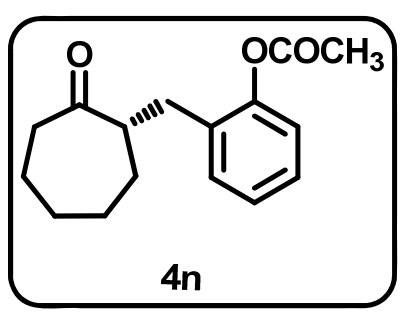

4n: Prepared according to the general procedure and obtained as colorless oil after purification by flash chromatography (15\% Et ${ }_{2} \mathrm{O} /$ petroleum ether). $45 \%$ yield, $95 \%$ ee. $[\alpha]_{\mathrm{D}}^{22}=+48.0\left(\mathrm{c}=0.6, \mathrm{CHCl}_{3}\right)$. HPLC analysis: Daicel Chiralpak OD-H, $5 \%$ iso-propanol/hexane, flow rate $=1.0 \mathrm{~mL} / \mathrm{min}, \lambda=206 \mathrm{~nm}$, retention time: $9.11 \mathrm{~min}$ (major), $8.37 \mathrm{~min}$ (minor). ${ }^{1} \mathrm{H} \mathrm{NMR}\left(400 \mathrm{MHz}, \mathrm{CDCl}_{3}\right) \delta 7.25-$ $7.13(\mathrm{~m}, 3 \mathrm{H}), 7.05-7.00(\mathrm{~m}, 1 \mathrm{H}), 3.02(\mathrm{dd}, \mathrm{J}=14.0,5.6 \mathrm{~Hz}, 1 \mathrm{H}), 2.79-2.77(\mathrm{~m}$, $\left.{ }_{1} \mathrm{H}\right), 2.53-2.43\left(\mathrm{~m},{ }_{3} \mathrm{H}\right), 2.33\left(\mathrm{~s},{ }_{3} \mathrm{H}\right), 1.90-1.71(\mathrm{~m}, 4 \mathrm{H}), 1.68-1.61(\mathrm{~m}, 1 \mathrm{H}), 1.41-$ $1.28(\mathrm{~m}, 3 \mathrm{H}) .{ }^{13} \mathrm{C}$ NMR (100 $\left.\mathrm{MHz}, \mathrm{CDCl}_{3}\right) \delta 215.3,169.7,149.4,132.1,131.3,127.6$, 126.1, 122.6, 52.4, 43.2, 32.3, 30.6, 29.5, 28.7, 24.4, 21.1. IR (thin film, $\mathrm{cm}^{-1}$ ) 2928, 2854, 1764, 1699, 1489, 1453, 1369, 1261, 1209, 1172, 1129, 1092, 1040, 1012, 917, 802, 754. HRMS (ESI) calcd for $\mathrm{C}_{16} \mathrm{H}_{20} \mathrm{O}_{3} \mathrm{Na}^{+}: 283.1305$, found 283.1307 .

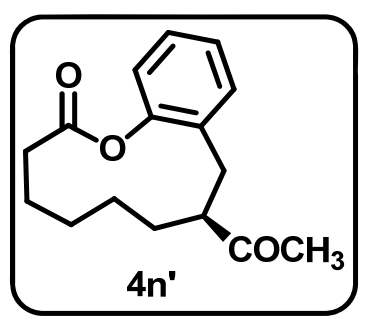

4n': Prepared according to the general procedure and obtained as colorless oil after purification by flash chromatography (20\% $\mathrm{Et}_{2} \mathrm{O} /$ petroleum ether). $46 \%$ yield, $98 \%$ ee. $[\alpha]_{\mathrm{D}}^{22}=+49.6\left(\mathrm{c}=1.0, \mathrm{CHCl}_{3}\right)$. HPLC analysis: Daicel Chiralpak $\mathrm{OJ}-\mathrm{H}, 5 \%$ iso-propanol/hexane, flow rate $=1.0 \mathrm{~mL} / \mathrm{min}, \lambda=205 \mathrm{~nm}$, retention time: $11.04 \mathrm{~min}$ (major), $10.18 \mathrm{~min}$ (minor). ${ }^{1} \mathrm{H}$ NMR (40o $\mathrm{MHz}, \mathrm{CDCl}_{3}$ ) $\delta 7.25-$ $7.18(\mathrm{~m}, 2 \mathrm{H}), 7.18$ - 7.07 (m, 2H), $2.96(\mathrm{dd}, \mathrm{J}=13.8,7.7 \mathrm{~Hz}, 1 \mathrm{H}), 2.85(\mathrm{dd}, \mathrm{J}=13.8$, $2.6 \mathrm{~Hz}, 1 \mathrm{H}), 2.80-2.72(\mathrm{~m}, 1 \mathrm{H}), 2.66(\mathrm{dt}, \mathrm{J}=14.2,5.1 \mathrm{~Hz}, 1 \mathrm{H}), 2.60-2.50(\mathrm{~m}, 1 \mathrm{H})$, 2.14 - $2.00(\mathrm{~m}, 4 \mathrm{H}), 1.88$ - $1.74(\mathrm{~m}, 2 \mathrm{H}), 1.70-1.44(\mathrm{~m}, 5 \mathrm{H}) .{ }^{13} \mathrm{C}$ NMR (100 MHz, $\left.\mathrm{CDCl}_{3}\right) \delta 210.8,171.7,148.7,133.6,131.5,127.5,126.1,122.7,52.6,32.7,29.6,29.3$, 28.3, 24.3, 22.8, 21.5. IR (thin film, $\mathrm{cm}^{-1}$ ) 2942, 2873, 1751, 1710, 1487, 1451, 1351, 1217, $1171,1157,1128,1104,1074,1019,802,750$. HRMS (ESI) calcd for $\mathrm{C}_{16} \mathrm{H}_{20} \mathrm{O}_{3} \mathrm{Na}^{+}$: 
283.1305, found 283.1306.

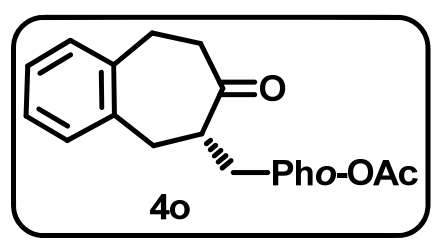

40: Prepared according to the general procedure and obtained as colorless oil after purification by flash chromatography ( $30 \% \mathrm{Et}_{2} \mathrm{O}$ /petroleum ether). $85 \%$ yield, $96 \%$ ee. $[\alpha]_{\mathrm{D}}^{22}=+21.8\left(\mathrm{c}=1.01, \mathrm{CHCl}_{3}\right)$. HPLC analysis: Daicel Chiralpak AS- $\mathrm{H}, 20 \%$ iso-propanol/hexane, flow rate $=1.0 \mathrm{~mL} / \mathrm{min}, \lambda=207 \mathrm{~nm}$, retention time: $9.47 \mathrm{~min}$ (major), $11.13 \mathrm{~min}$ (minor). ${ }^{1} \mathrm{H}$ NMR (50o $\left.\mathrm{MHz}, \mathrm{CD}_{3} \mathrm{OD}\right) \delta 7.29-$ $7.13(\mathrm{~m}, 6 \mathrm{H}), 7.07-7.01(\mathrm{~m}, 2 \mathrm{H}), 2.99-2.81(\mathrm{~m}, 5 \mathrm{H}), 2.77-2.64(\mathrm{~m}, 2 \mathrm{H}), 2.59-$ $2.46(\mathrm{~m}, 2 \mathrm{H}), 2.23(\mathrm{~s}, 3 \mathrm{H}) .{ }^{13} \mathrm{C}$ NMR (125 MHz, $\left.\mathrm{CD}_{3} \mathrm{OD}\right) \delta 214.3,171.1,150.8,141.8$, 140.1, 132.9, 131.9, 130.9, 130.1, 128.6, 128.3, 128.1, 127.1, 123.7, 54.6, 44.5, 37.1, 31.9, 31.8, 20.8. IR (thin film, $\mathrm{cm}^{-1}$ ) 2956, 2929, 1760, 1703, 1489, 1455, 1369, 1208, 1172, 1093, 1011, 919, 800, 751. HRMS (ESI) calcd for $\mathrm{C}_{20} \mathrm{H}_{20} \mathrm{O}_{3} \mathrm{Na}^{+}: 331.1305$, found 331.1304.

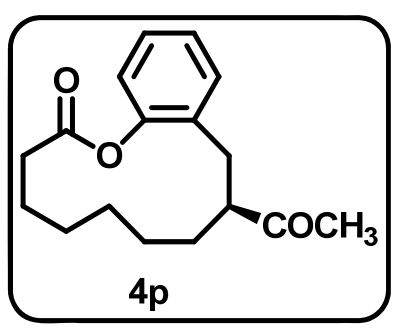

4P: Prepared according to the general procedure and obtained as colorless oil after purification by flash chromatography (20\% $\mathrm{Et}_{2} \mathrm{O} /$ petroleum ether). $24 \%$ yield, $98 \%$ ee. $[\alpha]_{\mathrm{D}}{ }^{22}=+16.0\left(\mathrm{c}=1.0, \mathrm{CHCl}_{3}\right)$. HPLC analysis: Daicel Chiralpak OD-H, $5 \%$ iso-propanol $/$ hexane, flow rate $=1.0 \mathrm{~mL} / \mathrm{min}, \lambda=205 \mathrm{~nm}$, retention time: $7.12 \mathrm{~min}$ (major), $7.10 \mathrm{~min}$ (minor). ${ }^{1} \mathrm{H}$ NMR (50o $\left.\mathrm{MHz}, \mathrm{CD}_{3} \mathrm{OD}\right) \delta 7.26-$ $7.19(\mathrm{~m}, 1 \mathrm{H}), 7.17(\mathrm{dd}, \mathrm{J}=7.6,1.7 \mathrm{~Hz}, 1 \mathrm{H}), 7.11(\mathrm{td}, \mathrm{J}=7.5,1.2 \mathrm{~Hz}, 1 \mathrm{H}), 7.02(\mathrm{dd}, \mathrm{J}=$ 8.1, 1.o Hz, 1H), 2.95 (dd, J = 13.3, $2.6 \mathrm{~Hz}, 1 \mathrm{H}), 2.87-2.74(\mathrm{~m}, 2 \mathrm{H}), 2.68(\mathrm{dd}, \mathrm{J}=$ 13.2, 10.1 Hz, $1 \mathrm{H}$ ), $2.48(\mathrm{ddd}, \mathrm{J}=14.7,10.4,2.3 \mathrm{~Hz}, 1 \mathrm{H}), 2.15-2.03(\mathrm{~m}, 1 \mathrm{H}), 1.97(\mathrm{~s}$, $3 \mathrm{H}), 1.94-1.82(\mathrm{~m}, 1 \mathrm{H}), 1.76-1.67(\mathrm{~m}, 2 \mathrm{H}), 1.60-1.42(\mathrm{~m}, 6 \mathrm{H}) .{ }^{13} \mathrm{C}$ NMR (125 $\left.\mathrm{MHz}, \mathrm{CD}_{3} \mathrm{OD}\right) \delta 213.8,174.9,150.7$, 134.1, 132.6, 128.6, 126.9, 124.0, 53.0, 36.o, 31.2, 
30.1, 29.8, 29.2, 25.9, 24.3, 23.4. IR (thin film, $\mathrm{cm}^{-1}$ ) 2934, 2870, 2859, 1750, 1712, $1489,1448,1351,1260,1215,1171,1128,1104,803,752$. HRMS (ESI) calcd for $\mathrm{C}_{17} \mathrm{H}_{22} \mathrm{O}_{3} \mathrm{Na}^{+}$: 297.1461, found 297.1461.

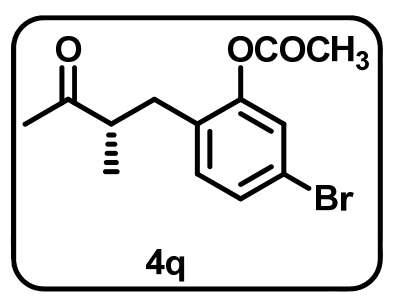

4q: Prepared according to the general procedure and obtained as colorless oil after purification by flash chromatography ( $20 \% \mathrm{Et}_{2} \mathrm{O} /$ petroleum ether). $50 \%$ yield, $94 \%$ ee. $[\alpha]_{\mathrm{D}}{ }^{22}=+15.8\left(\mathrm{c}=1.01, \mathrm{CHCl}_{3}\right)$. HPLC analysis: Daicel Chiralpak AS- $\mathrm{H}, 3 \%$ iso-propanol $/$ hexane, flow rate $=1.0 \mathrm{~mL} / \mathrm{min}, \lambda=204 \mathrm{~nm}$, retention time: 12.20 min (major), $18.28 \mathrm{~min}$ (minor). ${ }^{1} \mathrm{H} \mathrm{NMR}\left(400 \mathrm{MHz}, \mathrm{CDCl}_{3}\right) \delta 7.29$ $(\mathrm{dd}, \mathrm{J}=8.2,1.9 \mathrm{~Hz}, 1 \mathrm{H}), 7.23(\mathrm{~d}, \mathrm{~J}=1.9 \mathrm{~Hz}, 1 \mathrm{H}), 7.08(\mathrm{~d}, \mathrm{~J}=8.2 \mathrm{~Hz}, 1 \mathrm{H}), 2.89$ (dd, $J=13.8,6.8 \mathrm{~Hz}, 1 \mathrm{H}), 2.83-2.71(\mathrm{~m}, 1 \mathrm{H}), 2.43(\mathrm{dd}, J=13.8,7.3 \mathrm{~Hz}, 1 \mathrm{H}), 2.33(\mathrm{~s}, 3 \mathrm{H})$, $2.10\left(\mathrm{~s},{ }_{3} \mathrm{H}\right), 1.09(\mathrm{~d}, \mathrm{~J}=7.0 \mathrm{~Hz}, 3 \mathrm{H}) .{ }^{13} \mathrm{C}$ NMR (100 MHz, $\left.\mathrm{CDCl}_{3}\right) \delta$ 211.5, 169.1, 149.6, 132.2, 131.0, 129.4, 126.o, 120.2, 47.3, 32.8, 29.0, 21.0, 16.7. IR (thin film, $\mathrm{cm}^{-1}$ ) 2970, 2934, 1770, 1713, 1482, 1369, 1263, 1199, 1174, 1123, 1075, 1012, 925, 800. HRMS (ESI) calcd for $\mathrm{C}_{13} \mathrm{H}_{15} \mathrm{O}_{3} \mathrm{BrNa}^{+}$: 321.0097, found 321.0099.

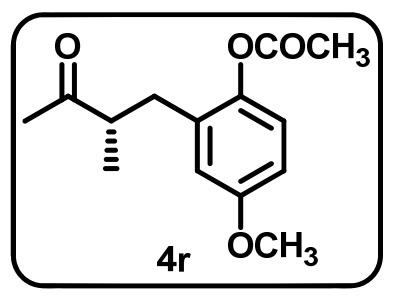

4r: Prepared according to the general procedure and obtained as colorless oil after purification by flash chromatography ( $25 \% \mathrm{Et}_{2} \mathrm{O}$ /petroleum ether). $79 \%$ yield, $96 \%$ ee. $[\alpha]_{D}^{22}=+15.7\left(\mathrm{c}=1.0, \mathrm{CHCl}_{3}\right)$. HPLC analysis: Daicel Chiralpak AS- $\mathrm{H}^{*} 2,3 \%$ iso-propanol $/$ hexane, flow rate $=1.0 \mathrm{~mL} / \mathrm{min}, \lambda=203 \mathrm{~nm}$, retention time: $36.89 \mathrm{~min}$ (major), $39.20 \mathrm{~min}$ (minor). ${ }^{1} \mathrm{H} \mathrm{NMR}\left(400 \mathrm{MHz}, \mathrm{CDCl}_{3}\right) \delta 6.95$ $(\mathrm{d}, \mathrm{J}=8.8 \mathrm{~Hz}, 1 \mathrm{H}), 6.8 \mathrm{o}-6.69(\mathrm{~m}, 2 \mathrm{H}), 3.78\left(\mathrm{~s},{ }_{3} \mathrm{H}\right), 2.89(\mathrm{dd}, \mathrm{J}=13.6,6.6 \mathrm{~Hz}$, $1 \mathrm{H}), 2.85-2.74(\mathrm{~m}, 1 \mathrm{H}), 2.43(\mathrm{dd}, \mathrm{J}=13.6,7.5 \mathrm{~Hz}, 1 \mathrm{H}), 2.31(\mathrm{~s}, 3 \mathrm{H}), 2.10(\mathrm{~s}, 3 \mathrm{H})$, $1.09(\mathrm{~d}, \mathrm{~J}=6.9 \mathrm{~Hz}, 3 \mathrm{H}) .{ }^{13} \mathrm{C}$ NMR $\left(100 \mathrm{MHz}, \mathrm{CDCl}_{3}\right) \delta 211.8,170.0,157.3,142.7$, 
132.8, 123.3, 116.2, 112.6, 55.6, 47.4, 33.5, 29.0, 21.0, 16.6. IR (thin film, $\mathrm{cm}^{-1}$ ) 2965, 2934, 1759, 1713, 1498, 1369, 1213,1195, 1180, 1033, 825, 803. HRMS (ESI) calcd for $\mathrm{C}_{14} \mathrm{H}_{18} \mathrm{O}_{4} \mathrm{Na}^{+}$: 273.1097, found 273.1099.

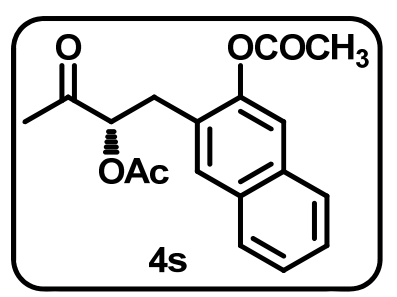

4s: Prepared according to the general procedure and obtained as colorless oil after purification by flash chromatography (50\% Et ${ }_{2} \mathrm{O} /$ petroleum ether). $99 \%$ yield, 92\% ee. $[\alpha]_{\mathrm{D}}^{22}=+33.8\left(\mathrm{c}=1.55, \mathrm{CHCl}_{3}\right)$. HPLC analysis: Daicel Chiralpak $\mathrm{AD}-\mathrm{H}, 2 \%$ iso-propanol/hexane, flow rate $=1.0 \mathrm{~mL} / \mathrm{min}, \lambda=225 \mathrm{~nm}$, retention time: $32.15 \mathrm{~min}$ (major), $34.79 \mathrm{~min}$ (minor). ${ }^{1} \mathrm{H} \mathrm{NMR}\left(500 \mathrm{MHz}, \mathrm{CDCl}_{3}\right) \delta 7.78$ $(\mathrm{dd}, \mathrm{J}=9.2,6.0 \mathrm{~Hz}, 2 \mathrm{H}), 7.72(\mathrm{~s}, 1 \mathrm{H}), 7.57(\mathrm{~s}, 1 \mathrm{H}), 7.50-7.41(\mathrm{~m}, 2 \mathrm{H}), 5.29(\mathrm{dd}, \mathrm{J}$ $=8.4,5.0 \mathrm{~Hz}, 1 \mathrm{H}), 3.22(\mathrm{dd}, \mathrm{J}=14.4$, 5.0 Hz, $1 \mathrm{H}), 3.08\left(\mathrm{dd}, \mathrm{J}=14.4,8.4 \mathrm{~Hz},{ }_{1} \mathrm{H}\right)$, $2.41(\mathrm{~s}, 3 \mathrm{H}), 2.11(\mathrm{~s}, 3 \mathrm{H}), 2.05(\mathrm{~s}, 3 \mathrm{H}) .{ }^{13} \mathrm{C} \mathrm{NMR}\left(125 \mathrm{MHz} \mathrm{CDCl}_{3}\right) \delta 205.4,170.5$, 169.5, 147.3, 133.1, 131.6, 130.6, 127.5, 127.5, 127.4, 126.6, 126.2, 120.0, 78.2, 31.9, 27.0, 21.2, 20.7. IR (thin film, $\mathrm{cm}^{-1}$ ) 2959, 2932, 1760, 1744, 1731, 1507, 1434, 1371, 1233, 1200, 1146, 1090, 1045, 1011, 923, 904, 750. HRMS (ESI) calcd for $\mathrm{C}_{18} \mathrm{H}_{18} \mathrm{O}_{5} \mathrm{Na}^{+}$: 337.1046, found 337.1045.

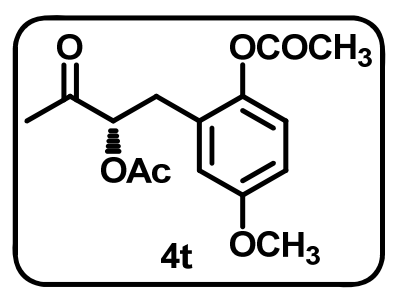

4t: Prepared according to the general procedure and obtained as colorless oil after purification by flash chromatography (40\% $\mathrm{Et}_{2} \mathrm{O} /$ petroleum ether). $72 \%$ yield, $92 \%$ ee. $[\alpha]_{\mathrm{D}}{ }^{22}=+4.4\left(\mathrm{c}=1.0, \mathrm{CHCl}_{3}\right)$. HPLC analysis: Daicel Chiralpak $\mathrm{AD}-\mathrm{H}, 10 \%$ iso-propanol/hexane, flow rate $=1.0 \mathrm{~mL} / \mathrm{min}, \lambda=204 \mathrm{~nm}$, retention time: $11.03 \mathrm{~min}$ (major), $10.35 \mathrm{~min}$ (minor). ${ }^{1} \mathrm{H}$ NMR (500 $\mathrm{MHz}, \mathrm{CDCl}_{3}$ ) $\delta 6.99$ (d, $J=8.8 \mathrm{~Hz}, 1 \mathrm{H}), 6.81(\mathrm{dd}, \mathrm{J}=8.8$, 3.0 Hz, $1 \mathrm{H}), 6.78(\mathrm{~d}, \mathrm{~J}=3.0 \mathrm{~Hz}, 1 \mathrm{H}), 5.17(\mathrm{dd}, \mathrm{J}=$ 8.4, 5.o Hz, $1 \mathrm{H}$ ), $3.78\left(\mathrm{~s},{ }_{3} \mathrm{H}\right), 3.00\left(\mathrm{dd}, \mathrm{J}=14.3,5.0 \mathrm{~Hz},{ }_{1} \mathrm{H}\right), 2.89(\mathrm{dd}, \mathrm{J}=14.3,8.4$ 
$\mathrm{Hz}, 1 \mathrm{H}), 2.33(\mathrm{~s}, 3 \mathrm{H}), 2.11(\mathrm{~s}, 3 \mathrm{H}), 2.09(\mathrm{~s}, 3 \mathrm{H}) .{ }^{13} \mathrm{C} \mathrm{NMR}\left(125 \mathrm{MHz}, \mathrm{CDCl}_{3}\right) \delta$ 205.2, 170.5, 169.8, 157.4, 142.9, 129.0, 123.5, 116.4, 113.5, 78.2, 55.7, 31.5, 26.9, 21.0, 20.7. IR (thin film, $\mathrm{cm}^{-1}$ ) 2968, 2943, 1763, 1744, 1731, 1500, 1429, 1372, 1238, 1196, 1179, 1046, 1036, 1011, 897, 817. HRMS (ESI) calcd for $\mathrm{C}_{15} \mathrm{H}_{18} \mathrm{O}_{6} \mathrm{Na}^{+}: 317.0996$, found 317.0995.

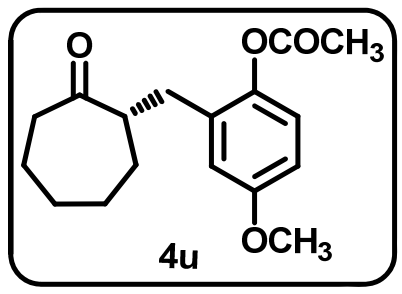

4u: Prepared according to the general procedure and obtained as colorless oil after purification by flash chromatography (20\% $\mathrm{Et}_{2} \mathrm{O} /$ petroleum ether). ${ }_{51} \%$ yield, $95 \%$ ee. $[\alpha]_{\mathrm{D}}^{22}=+35.3\left(\mathrm{c}=0.56, \mathrm{CHCl}_{3}\right)$. HPLC analysis: Daicel Chiralpak $\mathrm{AD}-\mathrm{H}^{*} 2,3 \%$ iso-propanol $/$ hexane, flow rate $=1.0 \mathrm{~mL} / \mathrm{min}, \lambda=203 \mathrm{~nm}$, retention time: $37.28 \mathrm{~min}$ (major), $38.91 \mathrm{~min}$ (minor). ${ }^{1} \mathrm{H} \mathrm{NMR}\left(400 \mathrm{MHz}, \mathrm{CD}_{3} \mathrm{OD}\right) \delta 6.95$ - $6.89(\mathrm{~m}, 1 \mathrm{H}), 6.8 \mathrm{o}-6.74(\mathrm{~m}, 2 \mathrm{H}), 3.77(\mathrm{~s}, 3 \mathrm{H}), 2.95-2.77(\mathrm{~m}, 2 \mathrm{H}), 2.52-2.38$ (m, $3 \mathrm{H}), 2.28(\mathrm{~s}, 3 \mathrm{H}), 1.91-1.74(\mathrm{~m}, 4 \mathrm{H}), 1.69-1.58(\mathrm{~m}, 1 \mathrm{H}), 1.38-1.35\left(\mathrm{~m},{ }_{3} \mathrm{H}\right)$. ${ }^{13} \mathrm{C}$ NMR (100 MHz, CD $3 \mathrm{OD}$ ) $\delta 217.7,171.7,158.8,144.2,134.3,124.3,117.1,113.4$, 56.0, 53.5, 43.9, 33.4, 31.6, 30.4, 29.6, 25.4, 20.8. IR (thin film, $\mathrm{cm}^{-1}$ ) 2965, 2927 , 2854, 1759, 1698, 1608, 1591, 1496, 1455, 1369, 126o, 1217, 1195, 1179, 1099, 1039, 1016, 80o. HRMS (ESI) calcd for $\mathrm{C}_{17} \mathrm{H}_{22} \mathrm{O}_{4} \mathrm{Na}^{+}$: 313.1410, found 313.1411.

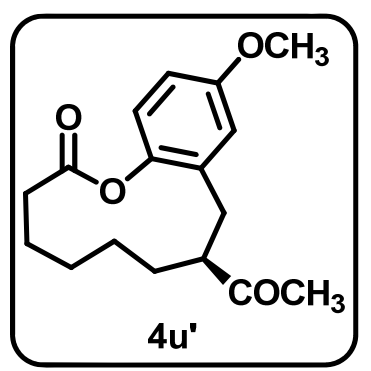

4u': Prepared according to the general procedure and obtained as colorless oil after purification by flash chromatography ( $25 \% \mathrm{Et}_{2} \mathrm{O} /$ petroleum ether). $42 \%$ yield, $98 \%$ ee. $[\alpha]_{\mathrm{D}}{ }^{22}=+12.3\left(\mathrm{c}=1.01, \mathrm{CHCl}_{3}\right)$. HPLC analysis: Daicel Chiralpak $\mathrm{OJ}-\mathrm{H}, 5 \%$ iso-propanol/hexane, flow rate $=1.0 \mathrm{~mL} / \mathrm{min}, \lambda=204 \mathrm{~nm}$, retention time: $24.84 \mathrm{~min}$ (major), $16.72 \mathrm{~min}$ (minor). ${ }^{1} \mathrm{H}$ NMR (40o $\mathrm{MHz}, \mathrm{CD}_{3} \mathrm{OD}$ ) $\delta 7.03$ $(\mathrm{d}, \mathrm{J}=8.8 \mathrm{~Hz}, 1 \mathrm{H}), 6.77(\mathrm{dd}, \mathrm{J}=8.8,3.0 \mathrm{~Hz}, 1 \mathrm{H}), 6.71(\mathrm{~d}, \mathrm{~J}=3.0 \mathrm{~Hz}, 1 \mathrm{H}), 3.74(\mathrm{~s}$, 
$3 \mathrm{H}), 2.85-2.81(\mathrm{~m}, 2 \mathrm{H}), 2.77-2.58(\mathrm{~m}, 3 \mathrm{H}), 2.07\left(\mathrm{~s},{ }_{3} \mathrm{H}\right), 2.05-1.96\left(\mathrm{~m},{ }_{1} \mathrm{H}\right), 1.86$ - $1.73(\mathrm{~m}, 2 \mathrm{H}), 1.70-1.44(\mathrm{~m}, 5 \mathrm{H}) .{ }^{13} \mathrm{C}$ NMR (100 MHz, $\left.\mathrm{CD}_{3} \mathrm{OD}\right) \delta$ 213.0, 173.7, 158.6, 143.5, 135.6, 124.5, 117.3, 113.2, 56.0, 53.7, 33.3, 30.3, 29.6, 29.1, 25.4, 23.6, 22.4. IR (thin film, cm $\mathrm{cm}^{-1}$ ) 2934, 2970, 1749, 1710, 1608, 1591, 1493, 1466, 1358, 1178, 1158, 1130, 1038, 803. HRMS (ESI) calcd for $\mathrm{C}_{17} \mathrm{H}_{22} \mathrm{O}_{4} \mathrm{Na}^{+}$: 313.1410, found 313.1412.

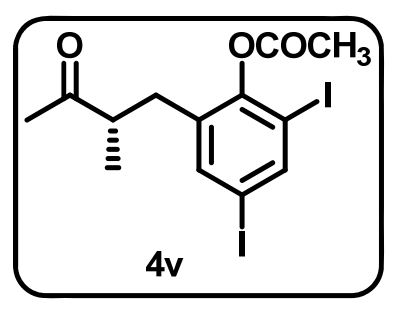

4v: Prepared according to the general procedure and obtained as colorless oil after purification by flash chromatography (20\% Et ${ }_{2} \mathrm{O} /$ petroleum ether). $44 \%$ yield, $95 \%$ ee. $[\alpha]_{\mathrm{D}}{ }^{22}=+8.8\left(\mathrm{c}=1.0, \mathrm{CHCl}_{3}\right)$. HPLC analysis: Daicel Chiralpak $\mathrm{OJ}-\mathrm{H}, 10 \%$ iso-propanol/hexane, flow rate $=1.0 \mathrm{~mL} / \mathrm{min}, \lambda=225 \mathrm{~nm}$, retention time: 17.97 min (major), 13.35 min (minor). ${ }^{1} \mathrm{H}$ NMR (50o $\left.\mathrm{MHz}, \mathrm{CDCl}_{3}\right) \delta 8.00(\mathrm{~d}$, $J=2.0 \mathrm{~Hz}, 1 \mathrm{H}), 7.50(\mathrm{~d}, J=2.0 \mathrm{~Hz}, 1 \mathrm{H}), 2.91(\mathrm{dd}, J=14.0,6.6 \mathrm{~Hz}, 1 \mathrm{H}), 2.81-2.71$ $(\mathrm{m}, 1 \mathrm{H}), 2.38(\mathrm{~s}, 3 \mathrm{H}), 2.12(\mathrm{~s}, 3 \mathrm{H}), 1.10(\mathrm{~d}, J=7.1 \mathrm{~Hz}, 3 \mathrm{H}) \cdot{ }^{13} \mathrm{C}$ NMR $(125 \mathrm{MHz}$, $\left.\mathrm{CDCl}_{3}\right) \delta 210.9,168.2,150.1,145.4,140.0,136.2$, 93.4, 91.3, 47.0, 33.6, 28.9, 21.3, 16.9. IR (thin film, $\mathrm{cm}^{-1}$ ) 2962, 2923, 1767, 1712, 1544, 1435, 1367, 1190, 1135, 1010, 900, 864, 797. HRMS (APCI) calcd for $\mathrm{C}_{13} \mathrm{H}_{14} \mathrm{O}_{3} \mathrm{I}_{2} \mathrm{Na}^{+}:$494.8925, found 494.8926 .

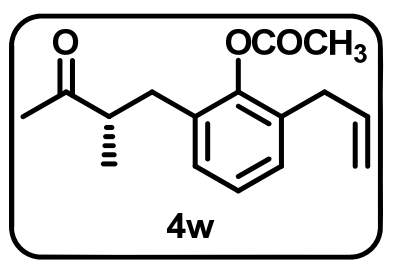

4w: Prepared according to the general procedure and obtained as colorless oil after purification by flash chromatography $\left(20 \% \mathrm{Et}_{2} \mathrm{O} /\right.$ petroleum ether). $73 \%$ yield, 94\% ee. $\quad[\alpha]_{\mathrm{D}}^{22}=+19.4\left(\mathrm{c}=1.01, \mathrm{CHCl}_{3}\right)$. HPLC analysis: Daicel Chiralpak AS-H, $3 \%$ iso-propanol/hexane, flow rate $=1.0 \mathrm{~mL} / \mathrm{min}, \lambda=203 \mathrm{~nm}$, retention time: $7.38 \mathrm{~min}$ (major), $9.00 \mathrm{~min}$ (minor). ${ }^{1} \mathrm{H}$ NMR (500 $\left.\mathrm{MHz}, \mathrm{CDCl}_{3}\right) \delta 7.13-$ $7.10(\mathrm{~m}, 2 \mathrm{H}), 7.09-7.05(\mathrm{~m}, 1 \mathrm{H}), 5.95-5.81(\mathrm{~m}, 1 \mathrm{H}), 5.11-5.03(\mathrm{~m}, 2 \mathrm{H}), 3.25(\mathrm{~d}, J$ $=6.7 \mathrm{~Hz}, 2 \mathrm{H}), 2.90(\mathrm{dd}, J=13.8,6.7 \mathrm{~Hz}, 1 \mathrm{H}), 2.86-2.76(\mathrm{~m}, 1 \mathrm{H}), 2.42(\mathrm{dd}, J=13.8$, 
$7.5 \mathrm{~Hz}, 1 \mathrm{H}), 2.33(\mathrm{~s}, 3 \mathrm{H}), 2.09(\mathrm{~s}, 3 \mathrm{H}), 1.09(\mathrm{~d}, J=7.0 \mathrm{~Hz}, 3 \mathrm{H}) .{ }^{13} \mathrm{C}$ NMR $(125 \mathrm{MHz}$, $\left.\mathrm{CDCl}_{3}\right) \delta 212.0,169.3,147.7,135.9,132.7,132.3,129.1,128.7,126.3,116.5,47.3,35.0$, 33.7, 29.0, 20.8, 16.7. IR (thin film, $\mathrm{cm}^{-1}$ ) 2967, 2929, 1760, 1713, 1457, 1370, 1209, 1161, 1011, 915, 797. HRMS (APCI) calcd for $\mathrm{C}_{16} \mathrm{H}_{20} \mathrm{O}_{3} \mathrm{Na}^{+}$: 283.1305, found 283.1307.

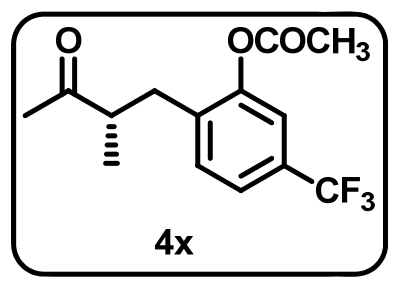

4X: Prepared according to the general procedure and obtained as colorless oil after purification by flash chromatography $\left(20 \% \mathrm{Et}_{2} \mathrm{O} /\right.$ petroleum ether). $50 \%$ yield, $97 \%$ ee. $[\alpha]_{\mathrm{D}}^{22}=+3.6\left(\mathrm{c}=1.01, \mathrm{CHCl}_{3}\right)$. HPLC analysis: Daicel Chiralpak AS-H, 3\% iso-propanol/hexane, flow rate $=1.0 \mathrm{~mL} / \mathrm{min}, \lambda=204 \mathrm{~nm}$, retention time: 8.51 min (major), $11.83 \min$ (minor). ${ }^{1} \mathrm{H}$ NMR (500 $\left.\mathrm{MHz}, \mathrm{CDCl}_{3}\right) \delta_{7.42}$ (d, J $=8.0 \mathrm{~Hz}, 1 \mathrm{H}), 7.33(\mathrm{~d}, \mathrm{~J}=8.3 \mathrm{~Hz}, 2 \mathrm{H}), 3.00(\mathrm{dd}, \mathrm{J}=13.9,6.9 \mathrm{~Hz}, 1 \mathrm{H}), 2.81(\mathrm{dd}, \mathrm{J}=$ 14.2, 7.1 Hz, $1 \mathrm{H}), 2.52(\mathrm{dd}, \mathrm{J}=13.9,7.3 \mathrm{~Hz}, 1 \mathrm{H}), 2.36(\mathrm{~s}, 3 \mathrm{H}), 2.11(\mathrm{~s}, 3 \mathrm{H}), 1.12(\mathrm{~d}, \mathrm{~J}=$ 7.1 Hz, $3 \mathrm{H}) \cdot{ }^{13} \mathrm{C}$ NMR (125 $\left.\mathrm{MHz} \mathrm{CDCl}_{3}\right){ }^{13} \mathrm{C} \mathrm{NMR}\left(125 \mathrm{MHz}, \mathrm{CDCl}_{3}\right) \delta$ 211.17, 169.09, 149.18, 136.23, 131.69, 130.55, 130.29, 130.03, 129.76, 126.90, 124.73, 123.00, $122.97,122.94,122.91,122.57,120.41,120.13,120.10,120.07,47.23,32.98,28.93,21.01$, 16.80. IR (thin film, $\mathrm{cm}^{-1}$ ) 2970, 2931, 1171, 1715, 1422, 1371, 1330, 1202, 1180, 1124, 1071, 1013, 939. HRMS (APCI) calcd for $\mathrm{C}_{14} \mathrm{H}_{15} \mathrm{O}_{3} \mathrm{~F}_{3} \mathrm{Na}^{+}$: 311.0866, found 311.0865 .

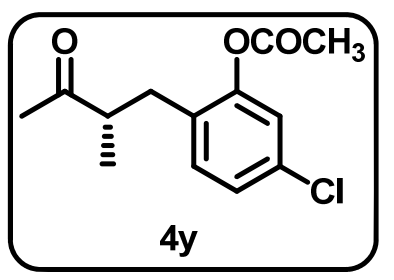

4y: Prepared according to the general procedure and obtained as colorless oil after purification by flash chromatography (20\% $\mathrm{Et}_{2} \mathrm{O} /$ petroleum ether). $51 \%$ yield, $98 \%$ ee. $[\alpha]_{\mathrm{D}}^{22}=+2.4\left(\mathrm{c}=0.98, \mathrm{CHCl}_{3}\right)$. HPLC analysis: Daicel Chiralpak $\mathrm{OJ}-\mathrm{H}, 3 \%$ iso-propanol/hexane, flow rate $=1.0 \mathrm{~mL} / \mathrm{min}, \lambda=203 \mathrm{~nm}$, retention time: $15.79 \min$ (major), $16.87 \mathrm{~min}$ (minor). ${ }^{1} \mathrm{H}$ NMR (40o $\left.\mathrm{MHz}, \mathrm{CDCl}_{3}\right) \delta 7.20-$ $7.11(\mathrm{~m}, 2 \mathrm{H}), 7.08(\mathrm{~s}, 1 \mathrm{H}), 2.91(\mathrm{dd}, \mathrm{J}=13.8,6.8 \mathrm{~Hz}, 1 \mathrm{H}), 2.77(\mathrm{dd}, \mathrm{J}=14.1,7.0 \mathrm{~Hz}$, 
$\left.{ }_{1 \mathrm{H}}\right), 2.46(\mathrm{~d}, \mathrm{~J}=7.3 \mathrm{~Hz}, 1 \mathrm{H}), 2.33\left(\mathrm{~s},{ }_{3} \mathrm{H}\right), 2.09\left(\mathrm{~s},{ }_{3} \mathrm{H}\right), 1.09\left(\mathrm{~d}, \mathrm{~J}=7.0 \mathrm{~Hz},{ }_{3} \mathrm{H}\right) . \mathrm{IR}$ (thin film, $\mathrm{cm}^{-1}$ ) 2970, 2929, 1768, 1714, 1487, 1369, 1261, 1200, 1175, 1081, 1013, 932, 799. ${ }^{13} \mathrm{C}$ NMR (100 MHz, $\mathrm{CDCl}_{3}$ ) $\delta 211.5,169.1,149.5,132.7$ 131.9, 130.5, 126.4, 123.2, 47.4, 32.8, 29.0, 21.0, 16.7. HRMS (APCI) calcd for $\mathrm{C}_{13} \mathrm{H}_{15} \mathrm{O}_{3} \mathrm{ClNa}^{+}$: 277.0602, found 277.0604 .

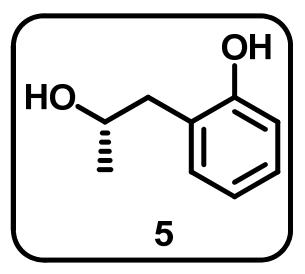

5: Prepared according to the literature report obtained as colorless oil after purification by flash chromatography $\left(50 \% \mathrm{Et}_{2} \mathrm{O} /\right.$ petroleum ether). ${ }^{11} 92 \%$ yield. $[\alpha]_{\mathrm{D}}^{22}=+5.2(\mathrm{c}=1.3, \mathrm{EtOH})$. The $(S)$-absolute configuration for the product $4 \mathrm{a}$ was determined by comparison of the optical rotation of $\mathbf{5}$ with the literature known product. ${ }^{12} \mathrm{H}$ NMR (40o $\left.\mathrm{MHz}, \mathrm{CDCl}_{3}\right) \delta 8.13(\mathrm{~s}, 1 \mathrm{H}), 7.19-7.10(\mathrm{~m}, 1 \mathrm{H})$, $7.02\left(\mathrm{dd}, \mathrm{J}=7.4,1.2 \mathrm{~Hz},{ }_{1} \mathrm{H}\right), 6.91\left(\mathrm{~d}, \mathrm{~J}=8.0 \mathrm{~Hz},{ }_{1} \mathrm{H}\right), 6.84\left(\mathrm{td}, \mathrm{J}=7.4,1.0 \mathrm{~Hz},{ }_{1} \mathrm{H}\right)$, $4.28-4.20(\mathrm{~m}, \mathrm{~J}=8.9,4.5 \mathrm{~Hz}, 1 \mathrm{H}), 2.86(\mathrm{dd}, \mathrm{J}=14.6,2.5 \mathrm{~Hz}, 1 \mathrm{H}), 2.77(\mathrm{dd}, \mathrm{J}=$ 14.6, 7.4 Hz, $1 \mathrm{H}), 2.46(\mathrm{~s}, 1 \mathrm{H}), 1.27\left(\mathrm{~d}, \mathrm{~J}=6.2 \mathrm{~Hz},{ }_{3} \mathrm{H}\right) .{ }^{13} \mathrm{C} \mathrm{NMR}\left(100 \mathrm{MHz}, \mathrm{CDCl}_{3}\right.$ ) $\delta 155.7,131.8,128.5,125.3,120.5,117.3,70.6,40.7,23.4$.

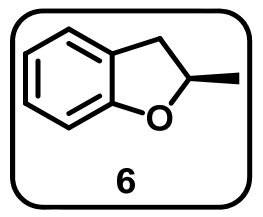

6: Prepared according to the literature report obtained as colorless oil after purification by flash chromatography $\left(5 \% \mathrm{Et}_{2} \mathrm{O} /\right.$ petroleum ether). ${ }^{13} 60 \%$ yield, 94\% ee. HPLC analysis: Daicel Chiralpak OJ-H, 1\% iso-propanol/hexane, flow rate $=0.5 \mathrm{~mL} / \mathrm{min}, \lambda=202 \mathrm{~nm}$, retention time: $12.32 \mathrm{~min}$ (major), $11.32 \mathrm{~min}$ (minor). ${ }^{1} \mathrm{H}$ NMR $\left(500 \mathrm{MHz}, \mathrm{CDCl}_{3}\right) \delta_{7.15}\left(\mathrm{~d}, \mathrm{~J}=7 \cdot 3 \mathrm{~Hz},{ }_{1} \mathrm{H}\right), 7.10(\mathrm{t}, \mathrm{J}=7.7 \mathrm{~Hz}$, $\left.{ }_{1 \mathrm{H}}\right), 6.82(\mathrm{td}, \mathrm{J}=7.4,0.8 \mathrm{~Hz}, 1 \mathrm{H}), 6.75\left(\mathrm{~d}, \mathrm{~J}=8.0 \mathrm{~Hz},{ }_{1} \mathrm{H}\right), 5.01-4.84(\mathrm{~m}, 1 \mathrm{H}), 3.31$ $(\mathrm{dd}, \mathrm{J}=15.4,8.8 \mathrm{~Hz}, 1 \mathrm{H}), 2.82(\mathrm{dd}, \mathrm{J}=15.4,7.7 \mathrm{~Hz}, 1 \mathrm{H}), 1.47\left(\mathrm{~d}, \mathrm{~J}=6.3 \mathrm{~Hz},{ }_{3} \mathrm{H}\right) .{ }^{13} \mathrm{C}$ NMR (125 MHz, $\left.\mathrm{CDCl}_{3}\right) \delta 159.6,128.1,127.2,125.1,120.3,109.5,79.6,37.3,21.9$. 


\section{Mechanistic studies}

\section{Scheme 1S. The Reaction Energy Profile by DFT}

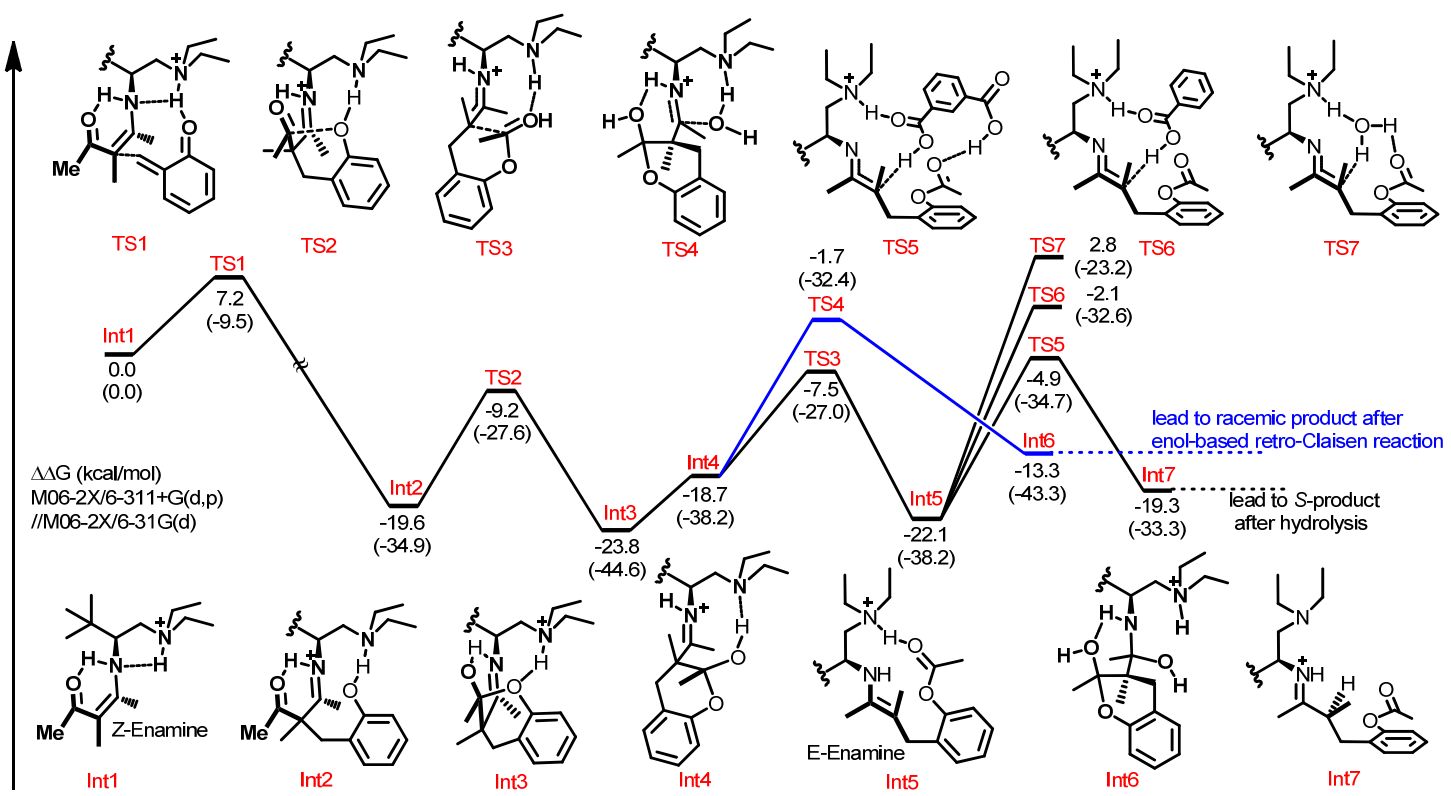

\section{Control experiments}

a) Reaction in the absence of aminocatalyst 1a: To a mixture of 1,3-diketons 2a (o.1 mmol), adipic acid (50 $\mathrm{mol} \%)$ and $\mathrm{KF}(0.11 \mathrm{mmol})$ in a standard glass tube were added the corresponding bromide 3 a $(0.11 \mathrm{mmol})$, followed by $0.7 \mathrm{~mL}$ dry $\mathrm{MeCN}$ via syringe. The resultant mixture was stirred at room temperature for 4oh. Solvent was removed and NMR of the crude mixture indicated a trace byproduct. $2 \mathrm{a}(\mathbf{8 . 5} \mathbf{~ m g})$ was recovered by column chromatograph.

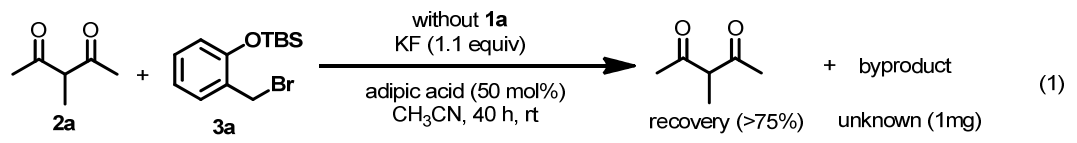

b) Treatment of product 4 a under catalytic conditions. To a mixture of the product rac-4a or $(S)-4 a(0.1 \mathrm{mmol})$, chiral amine $1 \mathbf{a}(20 \mathrm{~mol} \%)$, adipic acid (50 mol\%) and KF (o.11 mmol) in a standard glass tube were added the corresponding bromide za (o.11 mmol), followed by $0.7 \mathrm{~mL}$ dry $\mathrm{MeCN}$ via syringe. The resultant mixture was stirred at room temperature for 18-38 h. Solvent was removed and purification by silica gel column to recover 4 a $(>90 \%$ yield). 


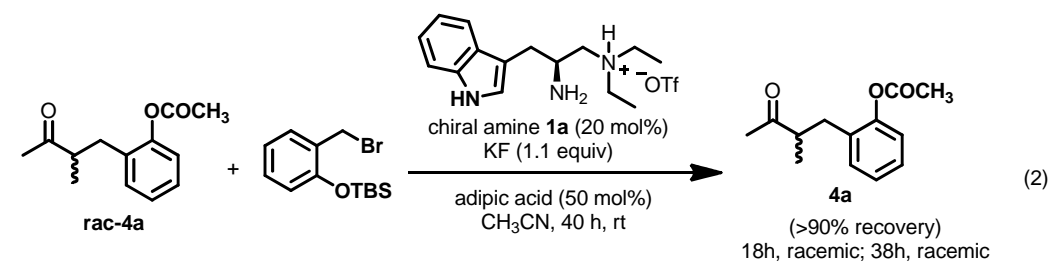

In-situ ESI- HRMS analysis of reaction mixture

a) Without $\mathbf{m}$-phthalic acid: To a mixture of 1,3-diketons 2 a (o.1 mmol), chiral amine $\mathbf{1 b}(20 \mathrm{~mol} \%)$, and $\mathrm{KF}(\mathrm{o.11} \mathrm{mmol})$ in a standard glass tube were added the corresponding bromide za (o.11 mmol), followed by $0.7 \mathrm{~mL}$ dry $\mathrm{MeCN}$ via syringe. After $2 \mathrm{~h}, 50 \mathrm{uL}$ of the reaction solution was taken, diluted with $1.0 \mathrm{~mL}$ of $\mathrm{CH}_{3} \mathrm{CN}$, and injected to the mass spectrometer. Positive mode was used. ESI-HRMS conditions: flow rate: $0.2 \mathrm{~mL} / \mathrm{min}$, solvent: $\mathrm{CH}_{3} \mathrm{CN}$.

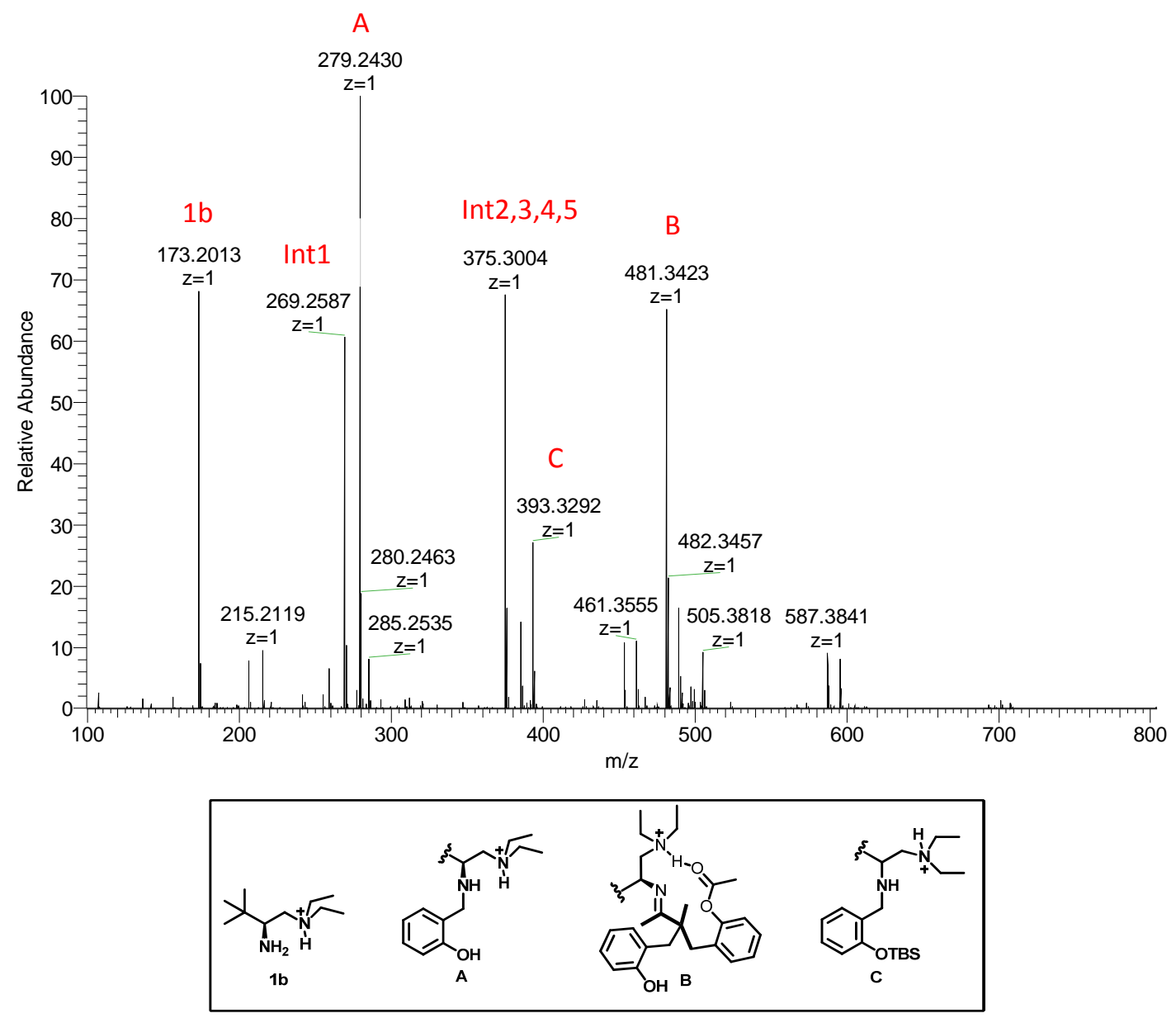

\begin{tabular}{|c|c|c|c|c|}
\hline Peak & Mass & $\begin{array}{c}\text { Expected } \\
\text { Formula }\end{array}$ & $\begin{array}{c}\text { Calculated } \\
\text { Mass }\end{array}$ & Difference \\
\hline 1b & 173.2013 & $\mathrm{C}_{10} \mathrm{H}_{25} \mathrm{~N}_{2}^{+}$ & 173.2012 & 0.0001 \\
\hline Int1 (Scheme 1) & 269.2587 & $\mathrm{C}_{16} \mathrm{H}_{33} \mathrm{~N}_{2} \mathrm{O}^{+}$ & 269.2587 & 0.0000 \\
\hline
\end{tabular}




\begin{tabular}{|c|l|l|l|l|}
\hline $\mathbf{A}$ & 279.2430 & $\mathrm{C}_{17} \mathrm{H}_{31} \mathrm{~N}_{2} \mathrm{O}^{+}$ & 279.2431 & 0.0001 \\
\hline Int2,3,4,5 (Scheme 1) & 375.3004 & $\mathrm{C}_{23} \mathrm{H}_{39} \mathrm{~N}_{2} \mathrm{O}_{2}{ }^{+}$ & 375.3006 & 0.0002 \\
\hline $\mathbf{C}$ & 393.3292 & $\mathrm{C}_{23} \mathrm{H}_{45} \mathrm{~N}_{2} \mathrm{OSi}^{+}$ & 393.3296 & 0.0004 \\
\hline $\mathbf{B}$ & 481.3423 & $\mathrm{C}_{30} \mathrm{H}_{45} \mathrm{~N}_{2} \mathrm{O}_{3}{ }^{+}$ & 481.3425 & 0.0002 \\
\hline
\end{tabular}

b) With $\boldsymbol{m}$-phthalic acid: To a mixture of 1,3-diketons 2 a (o.1 $\mathrm{mmol}$ ), chiral amine $\mathbf{1 b}(20 \mathrm{~mol} \%), \mathrm{m}$-phthalic acid (50 mol\%) and $\mathrm{KF}$ (o.11 $\mathrm{mmol}$ ) in a standard glass tube were added the corresponding bromide $3 \mathbf{a}$ ( $0.11 \mathrm{mmol})$, followed by $0.7 \mathrm{~mL}$ dry $\mathrm{MeCN}$ via syringe. After $2 \mathrm{~h}$, $50 \mathrm{uL}$ of the reaction solution was taken, diluted with 1.o $\mathrm{mL}$ of $\mathrm{CH}_{3} \mathrm{CN}$, and injected to the mass spectrometer. Positive mode was used. ESI-HRMS conditions: flow rate: 0.2 $\mathrm{mL} / \mathrm{min}$, solvent: $\mathrm{CH}_{3} \mathrm{CN}$.

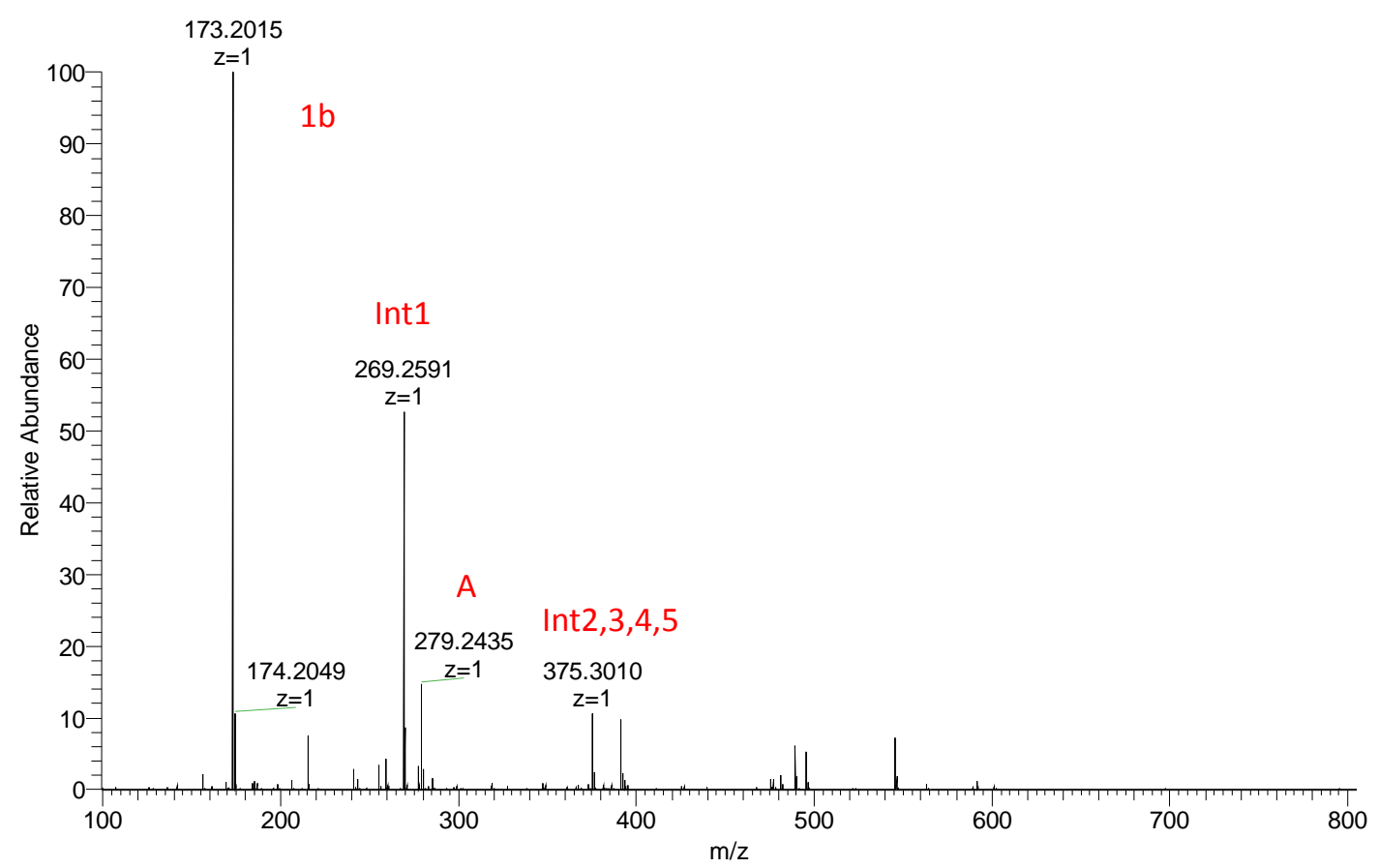

In-situ analysis of the reaction mixture by ESI-HRMS in the absence of m-phthalic acid revealed several by-products (A and $\mathbf{C})$ arisen from the poison of aminocatalyst. A notable peak @481.3423, could also be identified to be a double benzylated adduct $\mathbf{B}$, arisen from further benzylation with the enamine intermediate int5. This observation suggests that enamine protonation would be disfavored in the absence of weak acid, to note that no formation of $\mathbf{B}$ was detected in the presence of phthalic acid. Thus, one role of weak acid is to provide a favorable acid-base buffer system to suppress the side pathways. 


\section{Stoichiometric Reactions}

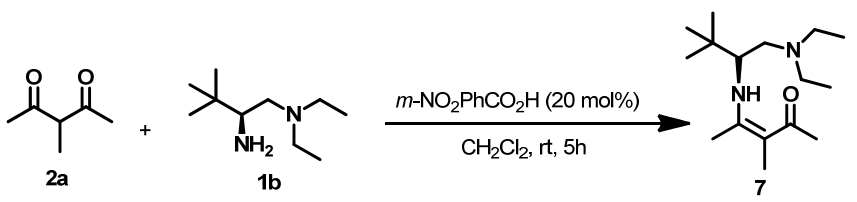

Synthesis of enamine intermediate 7: To $\beta$-diketone $2 \mathrm{a}(1 \mathrm{mmol})$, chiral primary amine $\mathbf{1 b}(1 \mathrm{mmol})$ in $\mathrm{CH}_{2} \mathrm{Cl}_{2}(0.5 \mathrm{~mL})$, was added $m$-nitrobenzoic acid (o.2 equiv). The reaction was stirred at room temperature for 5 h and directly purified by basic alumina column with $20 \% \mathrm{Et}_{2} \mathrm{O} /$ petroleum ether to give the enamine 7 as colorless oil. 7: ${ }^{1} \mathrm{H}$ NMR $\left(500 \mathrm{MHz}, \mathrm{CDCl}_{3}\right) \delta 12.57(\mathrm{~d}, J=9.4 \mathrm{~Hz}$, $1 \mathrm{H}), 3.26(\mathrm{t}, J=9.5 \mathrm{~Hz}, 1 \mathrm{H}), 2.63(\mathrm{~d}, J=13.4 \mathrm{~Hz}, 1 \mathrm{H}), 2.53-2.46(\mathrm{~m}, 2 \mathrm{H}), 2.41-2.32$ $(\mathrm{m}, 3 \mathrm{H}), 2.13(\mathrm{~s}, 3 \mathrm{H}), 2.00(\mathrm{~s}, 3 \mathrm{H}), 1.85(\mathrm{~s}, 3 \mathrm{H}), 0.96(\mathrm{~s}, 9 \mathrm{H}), 0.93(\mathrm{t}, J=7.1 \mathrm{~Hz}$, 6H). ${ }^{13} \mathrm{C}$ NMR (125 MHz, $\mathrm{CDCl}_{3}$ ) $\delta$ 193.24, 163.79, 97.08, 62.57, 56.25, 48.17, 34.47, 28.52, 26.88, 15.89, 15.18, 12.35 .

Stoichiometric Reactions: To a solution of enamine 7 in dry $\mathrm{CH}_{2} \mathrm{Cl}_{2}$ was added $\mathrm{TfOH}$ (1.o equiv) dropwise with vigorous stirring under $\mathrm{N}_{2}$ at $\mathrm{o}{ }^{\circ} \mathrm{C}$. After $10 \mathrm{~min}$, the solvent was removed under reduced pressure to give 7 - TfOH as a white solid.

To a mixture of freshly prepared 7 -TfOH ( $20 \mathrm{~mol} \%$ ), acid (50 mol\% or none) and $\mathrm{KF}$ (0.11 mmol), followed by $0.3 \mathrm{~mL} \mathrm{CH}_{3} \mathrm{CN}$ in a standard glass tube, were added the corresponding bromide $3 \mathrm{a}(0.11 \mathrm{mmol})$ at $\mathrm{o}{ }^{\circ} \mathrm{C}$. The reaction was stirred for $3 \mathrm{~h}$ and then quenched by aqueous acetonitrile solution $\left(5 \mathrm{uL} \mathrm{H}_{2} \mathrm{O}\right.$ dissolved in $0.3 \mathrm{~mL}$ acetonitrile) via syringe pump for 5 h or by aqueous $\mathrm{NH}_{4} \mathrm{Cl}$ solution (10 $\mathrm{uL})$ directly. The solvent was removed and purified by silica gel column with $20 \% \mathrm{Et}_{2} \mathrm{O}$ /petroleum ether to give the desired product. 


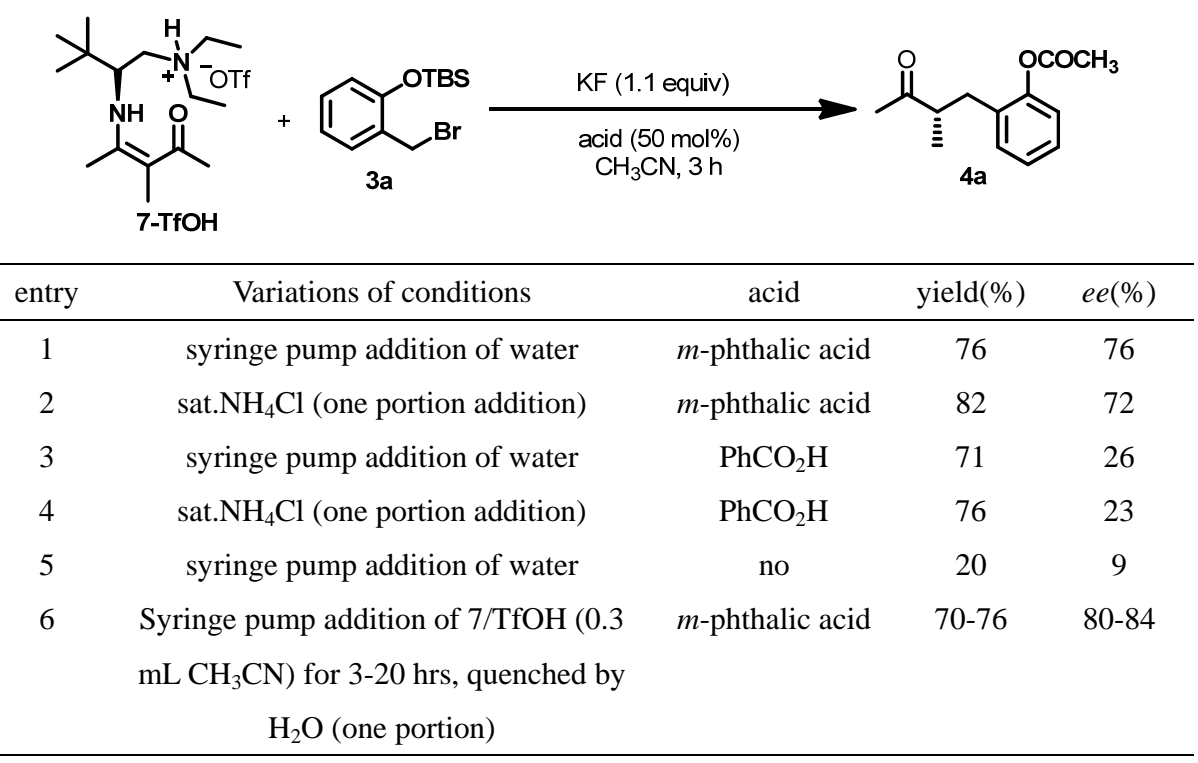

\section{Synthesis of enamine with diketone $2 b$ and $2 n$.}

To a standard glass tube containing 1,3-diketons $\mathbf{2 b}$ or $\mathbf{2 n}(0.2 \mathrm{mmol})$ in $0.5 \mathrm{~mL}$ $\mathrm{CH}_{3} \mathrm{CN}$ was added chiral primary amine $\mathbf{1}$ ( $0.2 \mathrm{mmol}$, 1.0 equiv) and adipic acid (20 mol\%). The reaction was stirred at room temperature for $3 \mathrm{~h}$ and directly purified by basic alumina column $\left(\mathrm{Et}_{2} \mathrm{O} /\right.$ petroleum ether) to give the desired enamine.

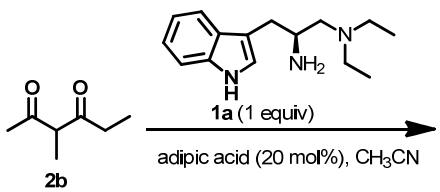<smiles>CCC(C(=O)CCCC(=O)C(CC)NC(C)(C)C)=C(C)NC(C)(C)C</smiles><smiles>CC(=O)C1CCCCC1=O</smiles>

$2 n$
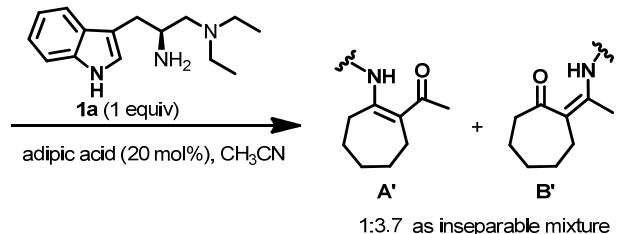

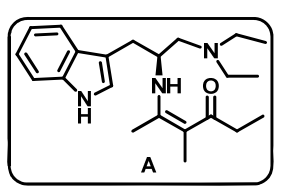

A: Prepared according to the general procedure and obtained as colorless oil after purification by flash chromatography $\left(\mathrm{Et}_{2} \mathrm{O} /\right.$ petroleum ether $\left.=4 / 1\right) \cdot 15 \%$ yield. ${ }^{1} \mathrm{H}$ NMR $\left(500 \mathrm{MHz}, \mathrm{CD}_{3} \mathrm{CN}\right) \delta 12.14(\mathrm{~d}, \mathrm{~J}=8.5 \mathrm{~Hz}, 1 \mathrm{H}), 9.09(\mathrm{~s}, 1 \mathrm{H}), 7.54(\mathrm{~d}$, $J=7.7 \mathrm{~Hz}, 1 \mathrm{H}), 7.37\left(\mathrm{~d}, \mathrm{~J}=8.1 \mathrm{~Hz},{ }_{1} \mathrm{H}\right), 7.14-7.07\left(\mathrm{~m},{ }_{1} \mathrm{H}\right), 7.04-6.98(\mathrm{~m}, 2 \mathrm{H})$, 
$3.97-3.87(\mathrm{~m}, 1 \mathrm{H}), 3.16-3.08(\mathrm{~m}, 1 \mathrm{H}), 2.74(\mathrm{dd}, \mathrm{J}=14.6,8.1 \mathrm{~Hz}, 1 \mathrm{H}), 2.55-2.43$ $(\mathrm{m}, 6 \mathrm{H}), 2.35(\mathrm{q}, \mathrm{J}=7.4 \mathrm{~Hz}, 2 \mathrm{H}), 1.74(\mathrm{~s}, 3 \mathrm{H}), 1.72(\mathrm{~s}, 3 \mathrm{H}), 1.00(\mathrm{t}, \mathrm{J}=7.4 \mathrm{~Hz}, 3 \mathrm{H})$, $0.95(\mathrm{t}, \mathrm{J}=7.1 \mathrm{~Hz}, 6 \mathrm{H}) .{ }^{13} \mathrm{C}$ NMR (125 MHz, CD $\left.\mathrm{CN}\right) \delta 197.5,162.8,137.4,128.7$, $124.4,122.4$, 119.8, 119.58, 113.0, 112.3, 97.7, 60.1, 54.7, 48.5, 33.4, 31.1, 15.8, 14.3, 12.4, $9 \cdot 9$.

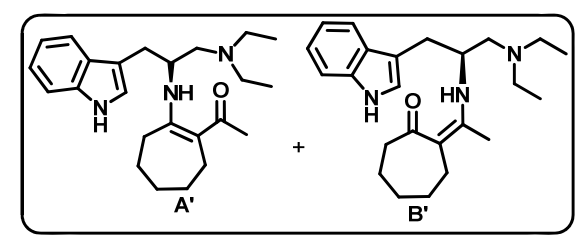

A'/ B'(inseparable mixture): Prepared according to the general procedure and obtained as colorless oil after purification by flash chromatography $\left(\mathrm{Et}_{2} \mathrm{O}\right) \cdot 72 \%$ yield. ${ }^{1} \mathrm{H}$ NMR (50o MHz, CD $\left.3 \mathrm{CN}\right) \delta 12.32\left(\mathrm{~d}, \mathrm{~J}=8.5 \mathrm{~Hz},{ }_{1} \mathrm{H}\right), 11.99(\mathrm{~d}, \mathrm{~J}=9.0 \mathrm{~Hz}$, ${ }_{1 H}$ ), $9.35(\mathrm{~s}, 1 \mathrm{H}), 7.53\left(\mathrm{~d}, \mathrm{~J}=7.9 \mathrm{~Hz},{ }_{1} \mathrm{H}\right), 7.36(\mathrm{~d}, \mathrm{~J}=8.1 \mathrm{~Hz}, 1 \mathrm{H}), 7.14$ - $7.07(\mathrm{~m}$, $\left.{ }_{1} \mathrm{H}\right), 7.05-6.97(\mathrm{~m}, 2 \mathrm{H}), 4.01-3.84(\mathrm{~m}, 1 \mathrm{H}), 3.18-3.06(\mathrm{~m}, 1 \mathrm{H}), 2.73(\mathrm{dt}, \mathrm{J}=14.5$, $7.2 \mathrm{~Hz}, 1 \mathrm{H}), 2.58$ - $2.42(\mathrm{~m}, 8 \mathrm{H}), 2.35$ - $2.32(\mathrm{~m}, 1 \mathrm{H}), 2.28-2.23(\mathrm{~m}, 2 \mathrm{H}), 2.05(\mathrm{~s}$, $1 \mathrm{H}), 1.73-1.64(\mathrm{~m}, 4 \mathrm{H}), 1.62-1.57(\mathrm{~m}, 2 \mathrm{H}), 1.50-1.32(\mathrm{~m}, 2 \mathrm{H}), 0.97-0.94(\mathrm{~m}, 6 \mathrm{H})$. ${ }^{13} \mathrm{C}$ NMR $\left(125 \mathrm{MHz}, \mathrm{CD}_{3} \mathrm{CN}\right) \delta 200.4,193.2,170.3,162.4,137.4,137.4,128.8,128.7$, $124.6,124.5,122.3,119.8,119.8,119.6,119.5,112.8,112.7,112.3,106.2,105.5,60.01$, $59.95,54.8,54.6,48.6,48.5,44.6,32.3,31.9,31.3,31.2,30.7,29.5,29.1,29.1,28.6$, $28.4,26.2,26.1,15.4,12.5,12.4$.

In-situ NMR characterization of enamine intermediate of $2 \mathrm{~b}$ and $2 \mathrm{n}$ : To a standard glass tube containing 1,3-diketons $2 \mathbf{b}$ or $\mathbf{2 n}(0.1 \mathrm{mmol})$ in $0.5 \mathrm{~mL}$ $\mathrm{CD}_{3} \mathrm{CN}$ was added chiral primary amine 1a/TfOH (o.1 mmol, 1.0 equiv) and adipic acid (20 mol\%). The reaction was stirred at room temperature for $6 \mathrm{~h}$ and directly characterized by in-situ ${ }^{1}$ HNMR.

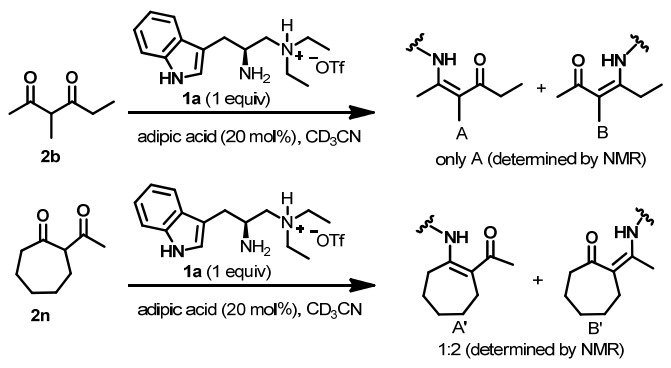


In-situ ${ }^{1} \mathrm{HNMR}$ of the reaction of $\mathbf{1 a} / \mathrm{TfOH}$ with $\mathbf{2 b}$

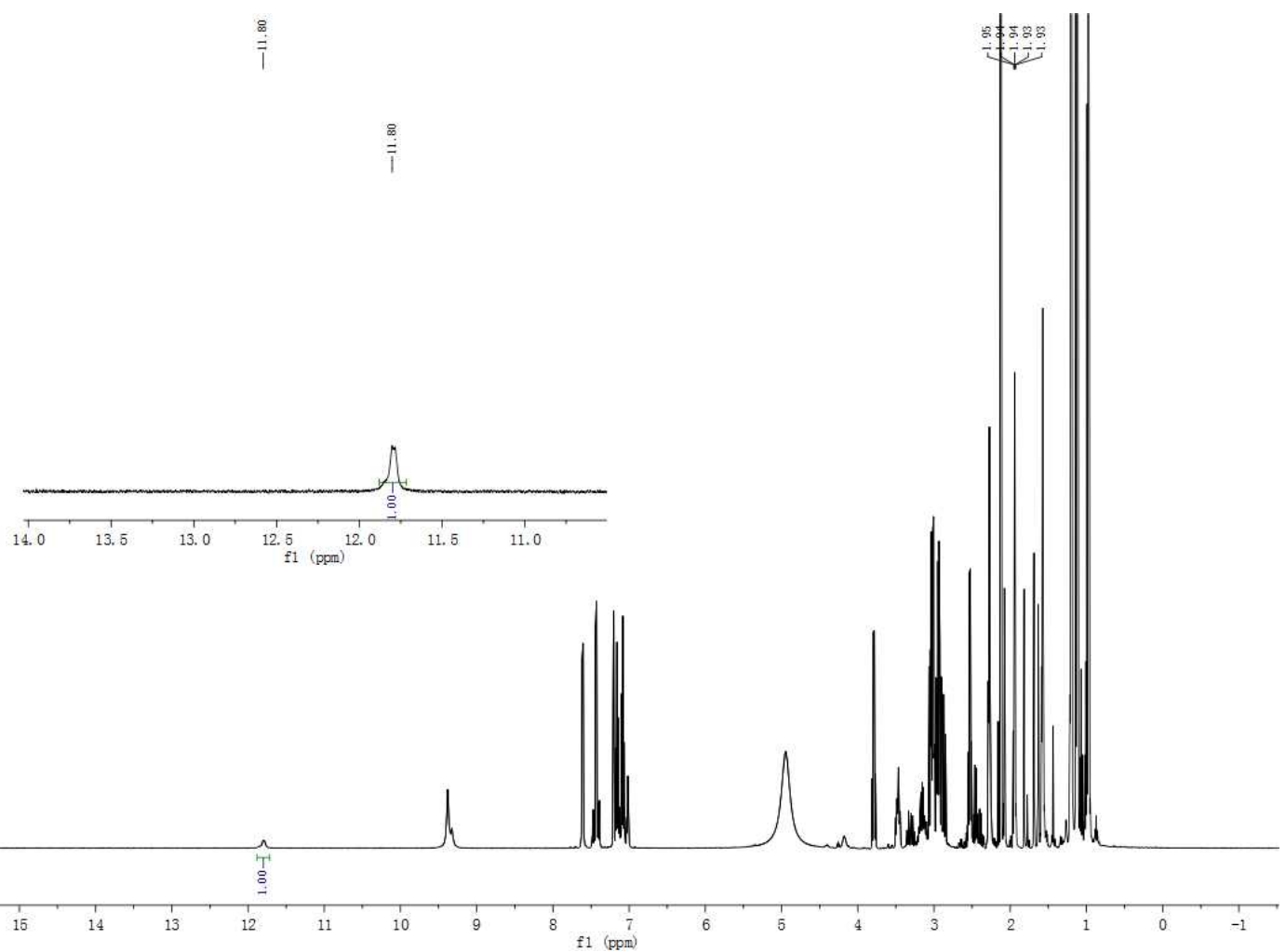

In-situ ${ }^{1} \mathrm{HNMR}$ of the reaction $\mathbf{1 a} / \mathrm{TfOH}$ with $\mathbf{2 n}$

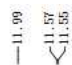
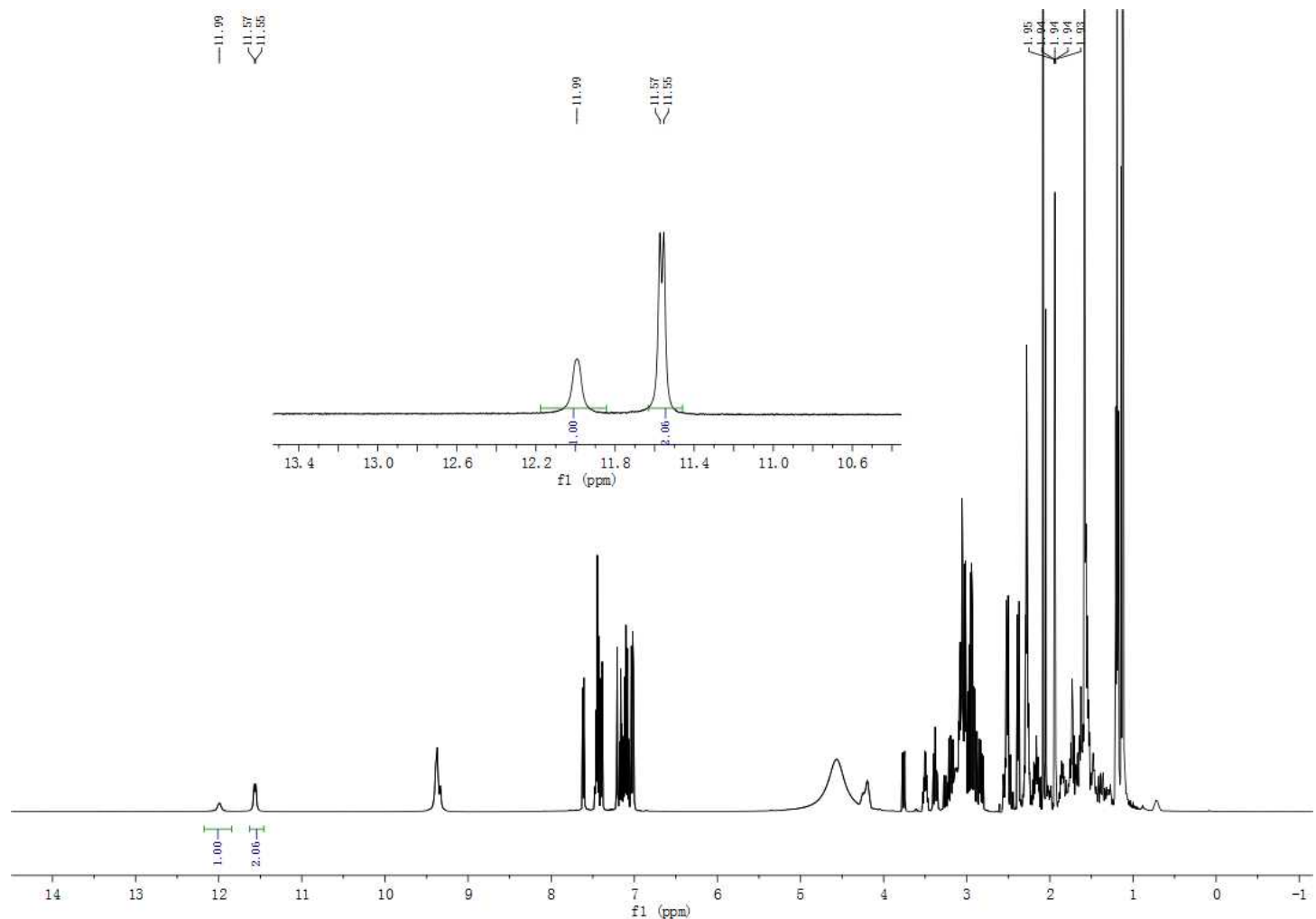


\section{Computational Studies}

DFT calculations were performed with the Gaussian o9 program package. ${ }^{14}$ The recently developed Mo6-2X functional ${ }^{15}$ together with the 6-31G(d) basis set were used for the geometry optimizations and vibrational calculations. Each geometry was confirmed as a minimum (no imaginary frequency) or a transition state (one imaginary frequency) by calculation of harmonic vibrational frequencies. The SMD continuum solvation $\operatorname{model}^{16}$ with acetonitrile as the solvent were used in single point energy calculations and these calculations were performed at the Mo6-2x/6-311+G(d,p) level with gas phase optimized structures.

1. The Michael addition of enamine to o-QM and the following ketalization

We have clarified that the Z-enamine was mainly formed in the reaction mixture by X-ray, NMR as well as DFT calculations, ${ }^{17}$ the Michael addition of $Z$-enamine to quinone methide via $R e$-facial attack was promoted by protonated tertiary amine assisted hydrogen-bond activation, which was found to have the lowest barrier. Both of the Si-facial attacking of $Z$ - and E-enamines were higher in energy. The following ketalization was very facial and a much stable ketal intermediate was formed. 

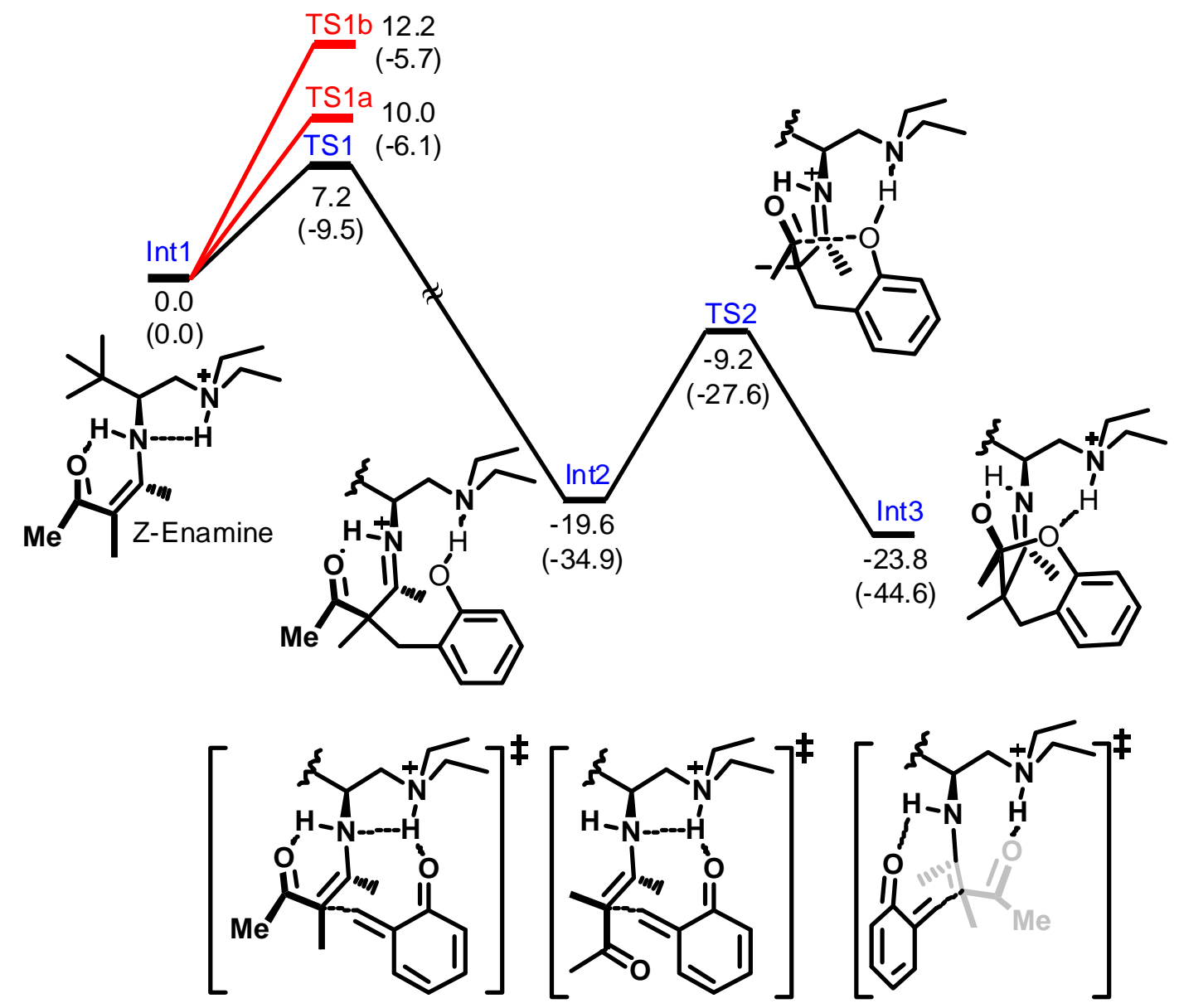

TS1: Re-facial attack of Z-enamine

$\Delta \Delta \mathrm{G}^{\ddagger}(\mathrm{kcal} / \mathrm{mol})$ $\Delta \Delta \mathrm{H}^{\ddagger}(\mathrm{kcal} / \mathrm{mol})$
0.0

$(0.0)$
TS1a: Si-facial attack of E-enamine

2.8

(3.4)
TS1b: Si-facial attack of Z-enamine

$$
5.0
$$

(3.8)

\section{The retro-Claisen process}

Unlike the classical retro-Claisen process, the C-C bond cleavage of int 3 via six-membered ring transition state (direct proton transfer from $\mathrm{O}-\mathrm{H}$ to imine) could not be found. Instead, a C-C bond rotation was occurred first to form the less stable ketal int4, then the $\mathrm{C}-\mathrm{C}$ bond cleavage proceeded via a crown-like nine-membered ring TS where the tertiary amine assisted the proton abstraction of ketal O-H, E-enamine was formed following this process. The intza could also be formed via a conformation change of int3, however, both of the direct retro claisen step via six-membered ring $\mathbf{T S}_{\mathbf{3}} \mathbf{b}$ and the tertiary amine assisted retro-Claisen step via nine-membered ring TS3a were much higher in energy than TS3. 


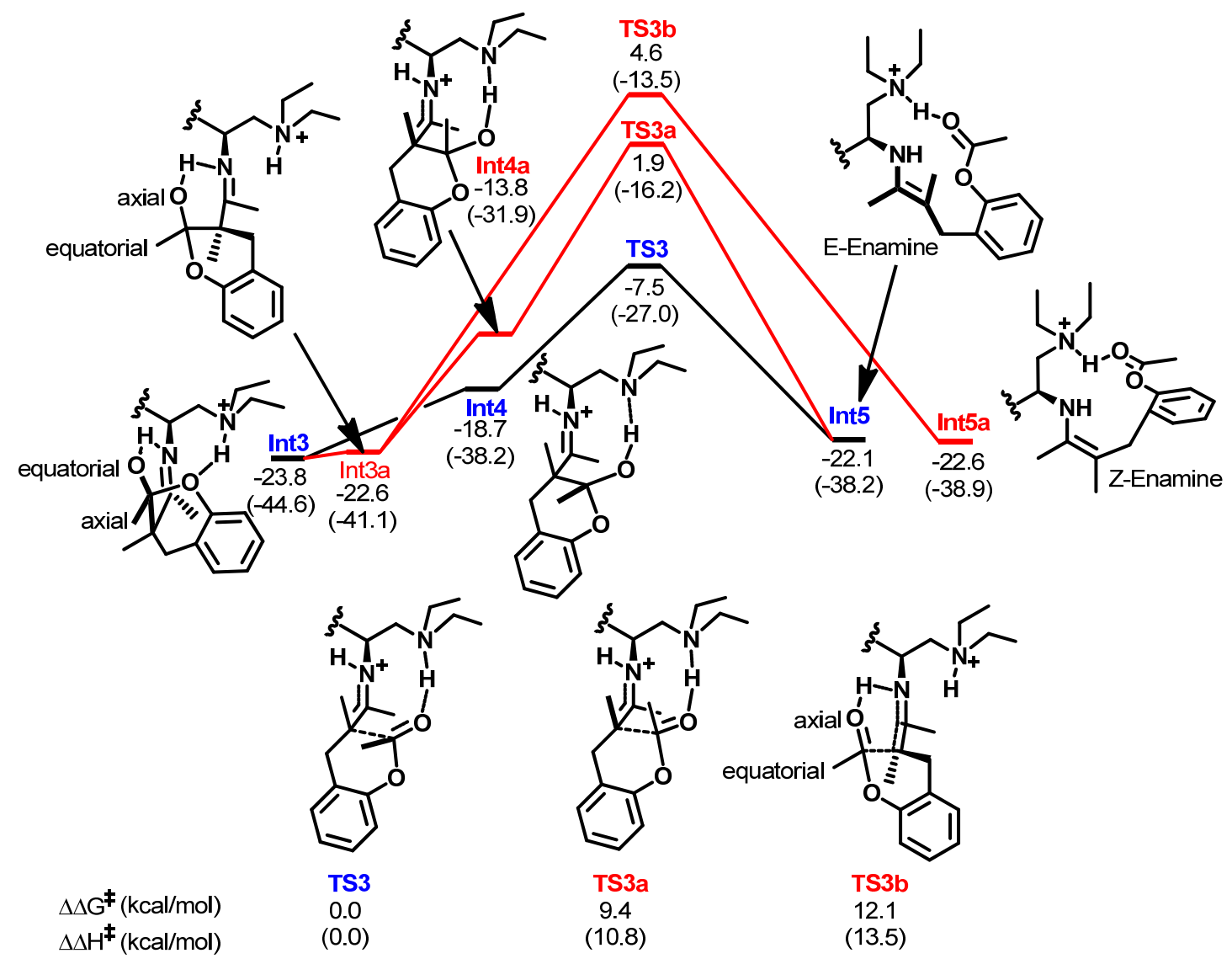

\section{The protonation and product hydrolysis process}

With E-enamine int5, the following protonation step was completed with the aid of a proton shuttle. Both water and weak acid could act as a proton shuttle, and the dicarboxylic acid shutlle gave the lowest activation energy. All of these TSs produced a stereocenter with $S$-configuration, and the $S$-product was finally formed after rapid hydrolysis process along with the release of primary amine catalyst. 

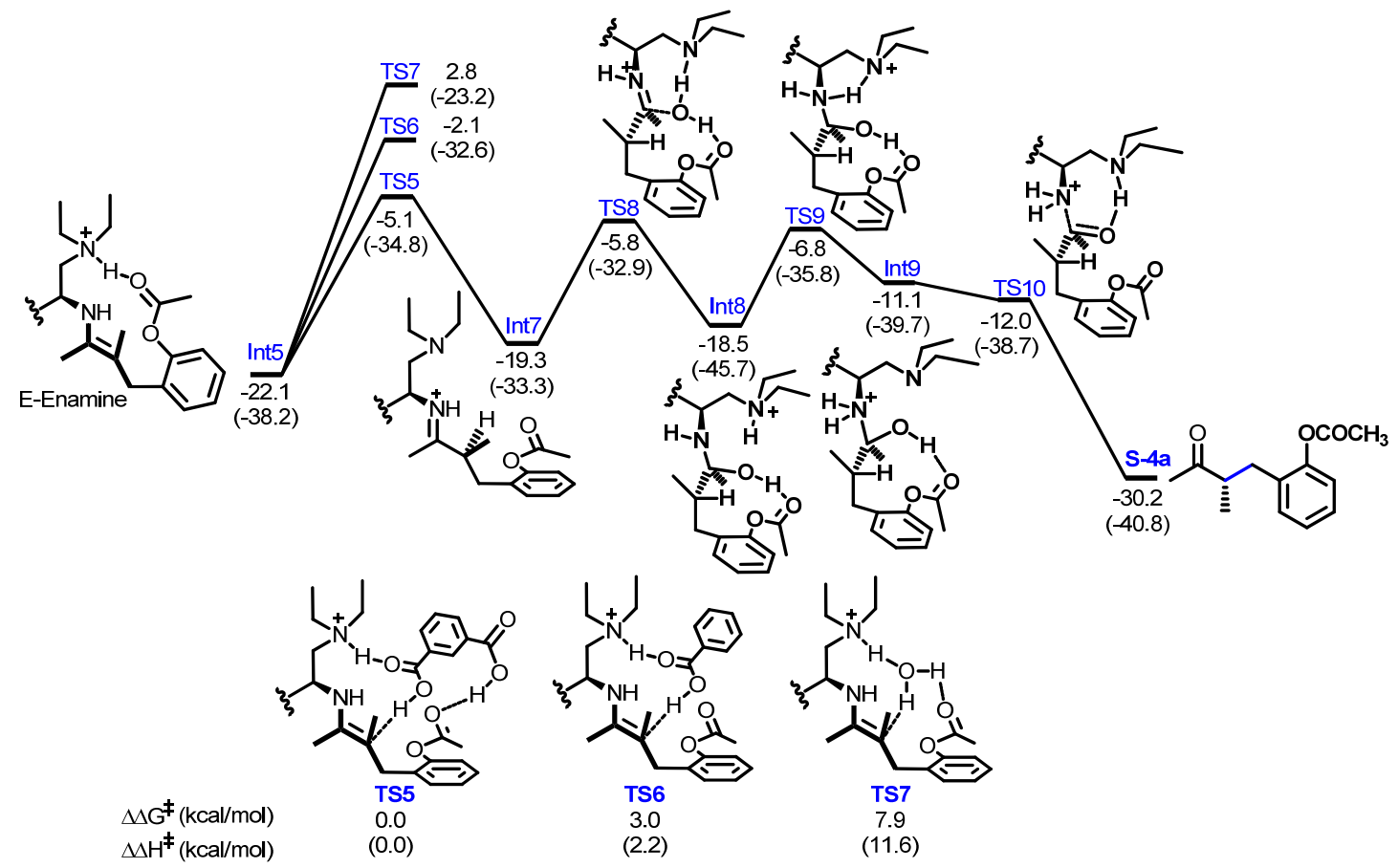

\section{The hydrolysis of ketal int3}

Besides the retro-Claisen process, the hydrolysis of int 3 could also occur with the activation energy of $16.2 \mathrm{kcal} / \mathrm{mol}$, after the release of catalyst, the residue would undergo enol-based retro-Claisen reaction to give the racemic product.

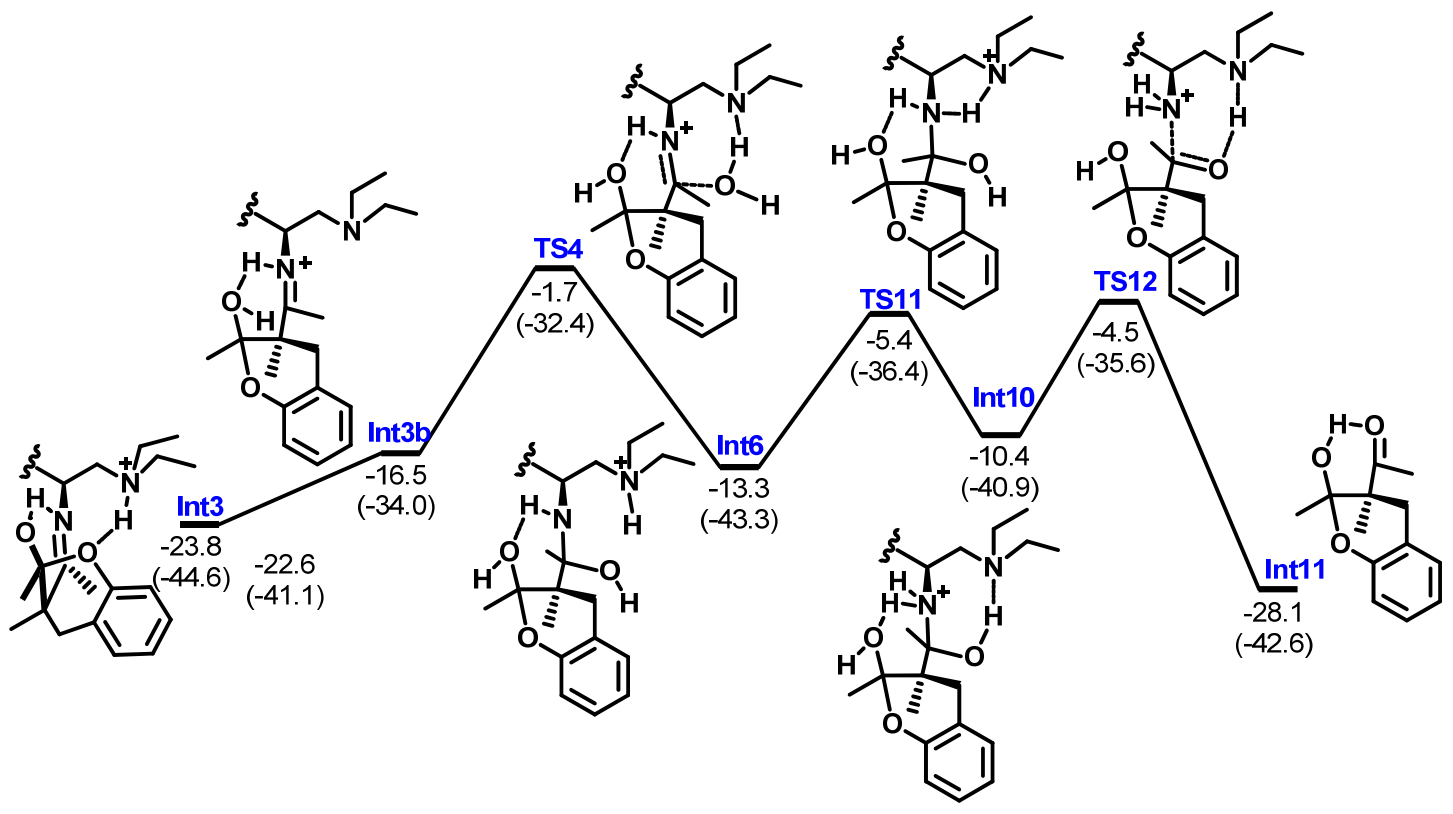




\section{The structure, energies and coordinates of intermediates and}

\section{transition states.}

(1) Int1

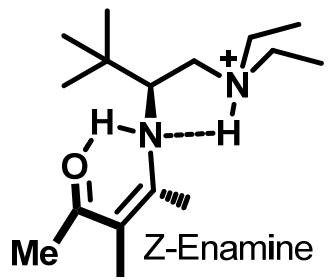

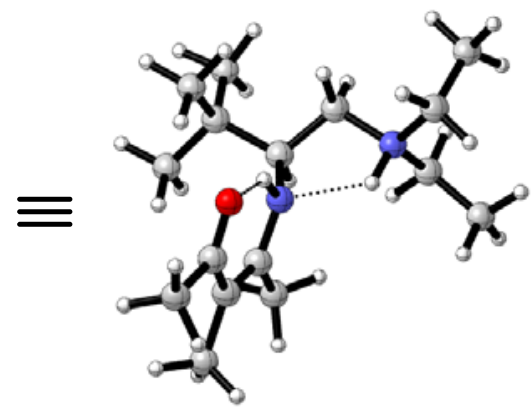

$\mathrm{E}_{\mathrm{sol}}=-814.0312336$

Zero-point correction=

0. 485646 (Hartree/Particle)

Thermal correction to Energy=

0.509309

Thermal correction to Enthalpy=

0.510253

Thermal correction to Gibbs Free Energy=

0.432794

Sum of electronic and zero-point Energies=

$-813.221465$

Sum of electronic and thermal Energies=

$-813.197802$

Sum of electronic and thermal Enthalpies=

$-813.196857$

Sum of electronic and thermal Free Energies=

$-813.274316$

E (Therma1)

$\mathrm{KCa} 1 / \mathrm{Mol}$

Total

Electronic

Translational

Rotational

Vibrational
319.596

0.000

0.889

0.889

317. 819
CV

Cal/Mol-Kelvin

88. 705

0.000

2. 981

2. 981

82.743
S

Cal/Mol-Kelvin

163. 026

0.000

42. 671

33. 922

86.433

Standard orientation:

\begin{tabular}{cccrrr} 
Center & Atomic & Atomic & \multicolumn{3}{c}{ Coordinates } \\
Number & Number & Type & X & Y & $Z$ \\
-1 & 6 & 0 & 0.004854 & 2.418100 & 0.165835 \\
1 & 6 & 0 & -1.101889 & 3.434279 & 0.481722 \\
2 & 1 & 0 & -1.518458 & 3.278852 & 1.484694 \\
3 & 1 & 0 & -0.693520 & 4.448453 & 0.449009 \\
4 & 1 & 0 & -1.920489 & 3.395341 & -0.245637 \\
5 & 6 & 0 & 1.197674 & 2.679644 & 1.093100 \\
7 & 1 & 0 & 0.901464 & 2.623343 & 2.147370 \\
8 & 1 & 0 & 2.011008 & 1.967827 & 0.916649
\end{tabular}




\begin{tabular}{|c|c|c|c|c|c|}
\hline 9 & 1 & 0 & 1.593625 & 3. 684099 & 0.915385 \\
\hline 10 & 6 & 0 & 0.459790 & 2. 583027 & -1.289287 \\
\hline & 1 & 0 & 1. 292549 & 1. 918209 & -1.539855 \\
\hline 10 & 1 & 0 & -0.348372 & 2. 408458 & -2.008724 \\
\hline e & 1 & 0 & 0.802475 & 3. 610428 & -1.445024 \\
\hline 14 & 6 & 0 & -0.526415 & 0.982275 & 0.447364 \\
\hline & 1 & 0 & -0.687853 & 0.909281 & 1. 529536 \\
\hline 16 & 6 & 0 & -1.871906 & 0.718553 & -0.262733 \\
\hline 17 & 1 & 0 & -1.802077 & 0.977815 & -1.322920 \\
\hline & 1 & 0 & -2.693566 & 1. 279840 & 0.184334 \\
\hline & 7 & 0 & 0.374965 & -0.093915 & 0.036147 \\
\hline 2 & 1 & 0 & -1.239758 & -1.168636 & -0.166111 \\
\hline 2 & 7 & 0 & -2.194767 & -0.746199 & -0.186619 \\
\hline & 6 & 0 & -2.900973 & -1.266849 & -1.410160 \\
\hline 2 & 1 & 0 & -2.267481 & -0.989675 & -2.256364 \\
\hline 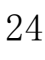 & 1 & 0 & -2.899620 & -2.356194 & -1.337223 \\
\hline & 6 & 0 & -2.870022 & -1.097105 & 1. 109304 \\
\hline & 1 & 0 & -2.251862 & -0.659948 & 1. 897412 \\
\hline 27 & 1 & 0 & -3.833750 & -0.582775 & 1. 116915 \\
\hline 28 & 6 & 0 & -3.017730 & -2.595119 & 1. 305863 \\
\hline & 1 & 0 & -3.741811 & -3.035507 & 0.616518 \\
\hline & 1 & 0 & -2.058336 & -3.110150 & 1. 191506 \\
\hline 3 & 1 & 0 & -3.375889 & -2.782274 & 2. 320648 \\
\hline & 6 & 0 & -4.307160 & -0.716839 & -1.562389 \\
\hline 3 & 1 & 0 & -4.319555 & 0.376617 & -1.583486 \\
\hline 2 & 1 & 0 & -4.715779 & -1.069050 & -2.512153 \\
\hline & 1 & 0 & -4.976035 & -1.064713 & -0.770900 \\
\hline & 1 & 0 & 0.703893 & -0.009782 & -0.935366 \\
\hline & 6 & 0 & 1. 420658 & -0.560299 & 0.838766 \\
\hline & 6 & 0 & 2. 610936 & -0.966838 & 0.305458 \\
\hline & 6 & 0 & 1. 114466 & -0.657328 & 2. 312171 \\
\hline & 1 & 0 & 0.056500 & -0.889398 & 2. 469229 \\
\hline & 1 & 0 & 1. 691759 & -1.448632 & 2. 787322 \\
\hline & 1 & 0 & 1. 331825 & 0.281241 & 2. 834657 \\
\hline & 6 & 0 & 3. 725083 & -1.506245 & 1. 175817 \\
\hline & 1 & 0 & 3. 641721 & -1.172356 & 2. 210128 \\
\hline & 1 & 0 & 3. 760088 & -2.602532 & 1. 176307 \\
\hline & 1 & 0 & 4. 696942 & -1.155439 & 0.821778 \\
\hline 4 & 6 & 0 & 2. 854366 & -0.900202 & -1.154289 \\
\hline & 8 & 0 & 2. 014467 & -0.487669 & -1.958068 \\
\hline & 6 & 0 & 4. 191686 & -1.361483 & -1.685481 \\
\hline & 1 & 0 & 4. 985821 & -0.675432 & -1.371028 \\
\hline & 1 & 0 & 4. 452563 & -2.355085 & -1.309223 \\
\hline & 1 & 0 & 4. 144355 & -1.375488 & -2.773901 \\
\hline
\end{tabular}


(2) TS1
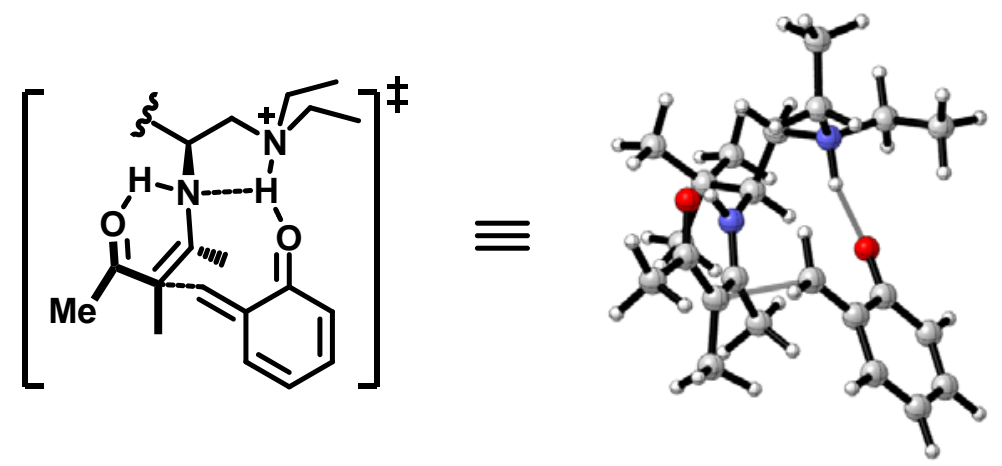

$\mathrm{E}_{\mathrm{sol}}=-1159.537356$

Zero-point correction=

0.598537 (Hartree/Particle)

Thermal correction to Energy=

0.628112

Thermal correction to Enthalpy=

0. 629057

Thermal correction to Gibbs Free Energy=

0.540407

Sum of electronic and zero-point Energies=

$-1158.525421$

Sum of electronic and thermal Energies=

$-1158.495845$

Sum of electronic and thermal Enthalpies=

$-1158.494901$

Sum of electronic and thermal Free Energies=

$-1158.583551$

\section{E (Therma1)}

KCa1/Mol

Total

394. 146

Electronic

Translational

Rotational

Vibrational
0. 000

0.889

0.889

392. 369
$\mathrm{CV}$

Cal/Mo1-Kelvin

115. 472

0. 000

2. 981

2. 981

109. 510
S

Cal/Mo1-Kelvin

186. 579

0.000

43. 660

35. 453

107. 466

Standard orientation:

\begin{tabular}{cccccc} 
Center & Atomic & Atomic & \multicolumn{3}{c}{ Coordinates } \\
Number & Number & Type & X & Y & $Z$ \\
$-----1 r o m s)$ & $Z$ \\
1 & 6 & 0 & -3.027860 & -0.957534 & -1.575458 \\
2 & 6 & 0 & -3.909902 & 0.001634 & -2.387434 \\
3 & 1 & 0 & -3.307828 & 0.636491 & -3.048182 \\
4 & 1 & 0 & -4.594000 & -0.573879 & -3.018061 \\
5 & 1 & 0 & -4.528403 & 0.645964 & -1.754952 \\
6 & 6 & 0 & -2.394269 & -1.964998 & -2.541612 \\
7 & 1 & 0 & -1.805669 & -1.465041 & -3.318420 \\
8 & 1 & 0 & -1.753346 & -2.681409 & -2.017574 \\
9 & 1 & 0 & -3.183454 & -2.533674 & -3.042245 \\
10 & 6 & 0 & -3.882314 & -1.720861 & -0.555476
\end{tabular}




\begin{tabular}{|c|c|c|c|c|c|}
\hline 11 & 1 & 0 & -3.270201 & -2.371489 & 0.079648 \\
\hline 12 & 1 & 0 & -4.463963 & -1.051820 & 0.087578 \\
\hline 13 & 1 & 0 & -4.599442 & -2.358097 & -1.081561 \\
\hline 14 & 6 & 0 & -1.900314 & -0.148491 & -0.865242 \\
\hline 15 & 1 & 0 & -1.337646 & 0.419495 & -1.616241 \\
\hline 16 & 6 & 0 & -2.472879 & 0.840794 & 0.157812 \\
\hline 17 & 1 & 0 & -2.936071 & 0.295023 & 0.984200 \\
\hline 18 & 1 & 0 & -3.235645 & 1. 475322 & -0.295133 \\
\hline 19 & 7 & 0 & -0.965326 & -0.984294 & -0.123891 \\
\hline 20 & 1 & 0 & -0.473819 & 1. 424346 & 0.331541 \\
\hline 21 & 7 & 0 & -1.404406 & 1. 728078 & 0.735610 \\
\hline 22 & 6 & 0 & -1.295171 & 1. 603738 & 2. 229967 \\
\hline 23 & 1 & 0 & -1.183526 & 0.533597 & 2. 435567 \\
\hline 24 & 1 & 0 & -0.361310 & 2. 094570 & 2. 515767 \\
\hline 25 & 6 & 0 & -1.553141 & 3. 142961 & 0.242784 \\
\hline 26 & 1 & 0 & -1.504165 & 3. 066710 & -0.846747 \\
\hline 27 & 1 & 0 & -2.551978 & 3. 485361 & 0.526631 \\
\hline 28 & 6 & 0 & -0.465185 & 4. 065172 & 0.760499 \\
\hline 29 & 1 & 0 & -0.551652 & 4. 249215 & 1. 834480 \\
\hline 30 & 1 & 0 & 0.523205 & 3. 657253 & 0.535083 \\
\hline 31 & 1 & 0 & -0.557754 & 5. 028874 & 0.254235 \\
\hline 32 & 6 & 0 & -2.480779 & 2. 199424 & 2. 968450 \\
\hline 33 & 1 & 0 & -3.431191 & 1. 773422 & 2. 633336 \\
\hline 34 & 1 & 0 & -2.378993 & 1. 978488 & 4. 033193 \\
\hline 35 & 1 & 0 & -2.528614 & 3. 285887 & 2. 858392 \\
\hline 36 & 1 & 0 & -1.223384 & -1.247918 & 0.834061 \\
\hline 37 & 6 & 0 & 0.224407 & -1.474117 & -0.485572 \\
\hline 38 & 6 & 0 & 1. 032493 & -2.170123 & 0.449714 \\
\hline 39 & 6 & 0 & 0.744301 & -1.294026 & -1.876969 \\
\hline 40 & 1 & 0 & 0.145148 & -0.612338 & -2.475431 \\
\hline 41 & 1 & 0 & 1. 769617 & -0.909526 & -1.839975 \\
\hline 42 & 1 & 0 & 0.785948 & -2.267125 & -2.379450 \\
\hline 43 & 6 & 0 & 2. 255493 & -2.892300 & -0.065774 \\
\hline 44 & 1 & 0 & 1. 999407 & -3.701354 & -0.760053 \\
\hline 45 & 1 & 0 & 2. 933374 & -2.210469 & -0.592535 \\
\hline 46 & 1 & 0 & 2. 827977 & -3.336026 & 0.749705 \\
\hline 47 & 6 & 0 & 0.511368 & -2.472403 & 1. 788028 \\
\hline 48 & 8 & 0 & -0.500017 & -1.919466 & 2. 246089 \\
\hline 49 & 6 & 0 & 4. 204277 & 1. 737535 & -1.793628 \\
\hline 50 & 6 & 0 & 4. 875231 & 0.845328 & -0.898051 \\
\hline 51 & 6 & 0 & 4. 159338 & 0.215216 & 0.070408 \\
\hline 52 & 6 & 0 & 2. 744167 & 0.438447 & 0.214253 \\
\hline 53 & 6 & 0 & 2. 051735 & 1. 329404 & -0.719857 \\
\hline 54 & 6 & 0 & 2. 862913 & 1. 978592 & -1.71430 \\
\hline
\end{tabular}




\begin{tabular}{rrrrrr}
55 & 1 & 0 & 4.786082 & 2.236607 & -2.563841 \\
56 & 1 & 0 & 5.942822 & 0.686954 & -0.997103 \\
57 & 1 & 0 & 4.643322 & -0.459607 & 0.772781 \\
58 & 1 & 0 & 2.361201 & 2.649092 & -2.403939 \\
59 & 8 & 0 & 0.800765 & 1.502074 & -0.686996 \\
60 & 6 & 0 & 2.023233 & -0.186941 & 1.209829 \\
61 & 1 & 0 & 2.551927 & -0.753174 & 1.973387 \\
62 & 1 & 0 & 1.014883 & 0.129675 & 1.460883 \\
63 & 6 & 0 & 1.251300 & -3.450584 & 2.672297 \\
64 & 1 & 0 & 1.349001 & -4.425935 & 2.186081 \\
65 & 1 & 0 & 2.263753 & -3.097827 & 2.898646 \\
66 & 1 & 0 & 0.695873 & -3.558205 & 3.603470 \\
\hline
\end{tabular}

\section{(3) TSia}

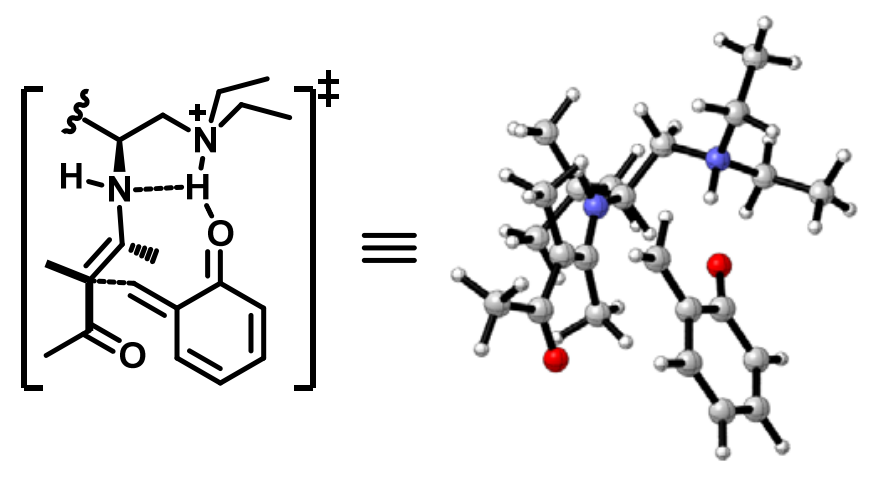

$\mathrm{E}_{\mathrm{sol}}=-1159.531346$

Zero-point correction=

0. 597635 (Hartree/Particle)

Thermal correction to Energy=

0.627491

Thermal correction to Enthalpy=

0.628435

Thermal correction to Gibbs Free Energy=

0.538739

Sum of electronic and zero-point Energies=

$-1158.509057$

Sum of electronic and thermal Energies=

Sum of electronic and thermal Enthalpies=

$-1158.479202$

$-1158.478258$

Sum of electronic and thermal Free Energies=

$-1158.567954$

$\begin{array}{lccc} & \text { E (Thermal) } & \text { CV } & \text { S } \\ & \text { KCal/Mol } & \text { Cal/Mol-Kelvin } & \text { Cal/Mo1-Kelvin } \\ \text { Total } & 393.756 & 116.342 & 188.782 \\ \text { Electronic } & 0.000 & 0.000 & 0.000 \\ \text { Translational } & 0.889 & 2.981 & 43.660 \\ \text { Rotational } & 0.889 & 2.981 & 35.545 \\ \text { Vibrational } & 391.979 & 110.380 & 109.576\end{array}$

Standard orientation:

\begin{tabular}{llccccc} 
Center & Atomic & Atomic & & \multicolumn{2}{c}{ Coordinates (Angstroms) } \\
Number & Number & Type & & X & Y & \\
& & & s33 & &
\end{tabular}




\begin{tabular}{|c|c|c|c|c|c|}
\hline 1 & 6 & 0 & -2.727811 & -1.966753 & -1.170705 \\
\hline 2 & 6 & 0 & -3.763319 & -1.364279 & -2.130293 \\
\hline 3 & 1 & 0 & -3.284040 & -0.721004 & -2.877519 \\
\hline 4 & 1 & 0 & -4.276196 & -2.167123 & -2.668017 \\
\hline 5 & 1 & 0 & -4.536581 & -0.782804 & -1.617884 \\
\hline 6 & 6 & 0 & -1.804385 & -2.888838 & -1.975134 \\
\hline 7 & 1 & 0 & -1.324859 & -2.358342 & -2.803925 \\
\hline 8 & 1 & 0 & -1.024349 & -3.330429 & -1.347009 \\
\hline 9 & 1 & 0 & -2.389867 & -3.707904 & -2.403315 \\
\hline 10 & 6 & 0 & -3.436195 & -2.791408 & -0.088267 \\
\hline 11 & 1 & 0 & -2.724648 & -3.233967 & 0.619534 \\
\hline 12 & 1 & 0 & -4.168818 & -2.202253 & 0.475371 \\
\hline 13 & 1 & 0 & -3.980400 & -3.619360 & -0.552047 \\
\hline 14 & 6 & 0 & -1.891242 & -0.818500 & -0.530664 \\
\hline 15 & 1 & 0 & -1.499820 & -0.179211 & -1.328850 \\
\hline 16 & 6 & 0 & -2.735094 & 0.054837 & 0.409111 \\
\hline 17 & 1 & 0 & -2.848478 & -0.438770 & 1. 379352 \\
\hline 18 & 1 & 0 & -3.736281 & 0.225294 & 0.010036 \\
\hline 19 & 7 & 0 & -0.751990 & -1.286856 & 0.251558 \\
\hline 20 & 1 & 0 & -1.064661 & 1. 319588 & 0.409280 \\
\hline 21 & 7 & 0 & -2.097977 & 1. 392626 & 0.641981 \\
\hline 22 & 6 & 0 & -2.188450 & 1. 839779 & 2. 070660 \\
\hline 23 & 1 & 0 & -1.746568 & 1. 031646 & 2. 661756 \\
\hline 24 & 1 & 0 & -1.538015 & 2. 711836 & 2. 166699 \\
\hline 25 & 6 & 0 & -2.619745 & 2. 403557 & -0.347616 \\
\hline 26 & 1 & 0 & -2.380601 & 1. 990569 & -1.331162 \\
\hline 27 & 1 & 0 & -3.707145 & 2.431711 & -0.236973 \\
\hline 28 & 6 & 0 & -1.998776 & 3. 778782 & -0.183643 \\
\hline 29 & 1 & 0 & -2.316902 & 4. 271255 & 0.739084 \\
\hline 30 & 1 & 0 & -0.909265 & 3. 710968 & -0.213917 \\
\hline 31 & 1 & 0 & -2.326023 & 4. 404314 & -1.017617 \\
\hline 32 & 6 & 0 & -3.607148 & 2. 143314 & 2. 518187 \\
\hline 33 & 1 & 0 & -4.274730 & 1. 288446 & 2. 373820 \\
\hline 34 & 1 & 0 & -3.600015 & 2. 381158 & 3. 584124 \\
\hline 35 & 1 & 0 & -4.024198 & 3. 005236 & 1. 991051 \\
\hline 36 & 1 & 0 & -0.998362 & -1.686754 & 1. 148321 \\
\hline 37 & 6 & 0 & 0.553565 & -1.397645 & -0.102548 \\
\hline 38 & 6 & 0 & 1. 465156 & -1.778416 & 0.894295 \\
\hline 39 & 6 & 0 & 0.968008 & -1.119574 & -1.511820 \\
\hline 40 & 1 & 0 & 0.145313 & -0.773725 & -2.132498 \\
\hline 41 & 1 & 0 & 1. 779167 & -0.389652 & -1.536199 \\
\hline 42 & 1 & 0 & 1. 391923 & -2.032847 & -1.941212 \\
\hline 43 & 6 & 0 & 0.927874 & -2.192456 & 2. 245825 \\
\hline
\end{tabular}




\begin{tabular}{|c|c|c|c|c|c|}
\hline 44 & 1 & 0 & 0.297995 & -1.408805 & 2. 691084 \\
\hline 45 & 1 & 0 & 0.326521 & -3.111156 & 2. 193670 \\
\hline 46 & 1 & 0 & 1. 728408 & -2.378911 & 2. 961518 \\
\hline 47 & 6 & 0 & 2. 866883 & -2.124305 & 0.532340 \\
\hline 48 & 8 & 0 & 3. 376469 & -1.755959 & -0.511622 \\
\hline 49 & 6 & 0 & 3. 411422 & 2. 721362 & -1.742053 \\
\hline 50 & 6 & 0 & 4. 336398 & 2. 055038 & -0.880630 \\
\hline 51 & 6 & 0 & 3. 861269 & 1. 288007 & 0.136399 \\
\hline 52 & 6 & 0 & 2. 448097 & 1. 158013 & 0.363920 \\
\hline 53 & 6 & 0 & 1. 500305 & 1. 797573 & -0.547653 \\
\hline 54 & 6 & 0 & 2. 057496 & 2. 609415 & -1.590835 \\
\hline 55 & 1 & 0 & 3. 802807 & 3. 331277 & -2.551983 \\
\hline 56 & 1 & 0 & 5. 401310 & 2. 162677 & -1.049586 \\
\hline 57 & 1 & 0 & 4. 538394 & 0.764635 & 0.806424 \\
\hline 58 & 1 & 0 & 1. 365449 & 3. 102089 & -2.265875 \\
\hline 59 & 8 & 0 & 0.249729 & 1. 618842 & -0.452645 \\
\hline 60 & 6 & 0 & 1. 950730 & 0.426170 & 1. 419646 \\
\hline 61 & 1 & 0 & 2. 621439 & 0.052278 & 2. 189816 \\
\hline 62 & 1 & 0 & 0.902741 & 0.521418 & 1. 691713 \\
\hline 63 & 6 & 0 & 3. 691492 & -2.917118 & 1. 530002 \\
\hline 64 & 1 & 0 & 3. 905514 & -2.322238 & 2. 426304 \\
\hline 65 & 1 & 0 & 3. 179188 & -3.828044 & 1. 853586 \\
\hline 66 & 1 & 0 & 4. 635665 & -3.175881 & 1. 051266 \\
\hline
\end{tabular}

\section{(4) TSib}

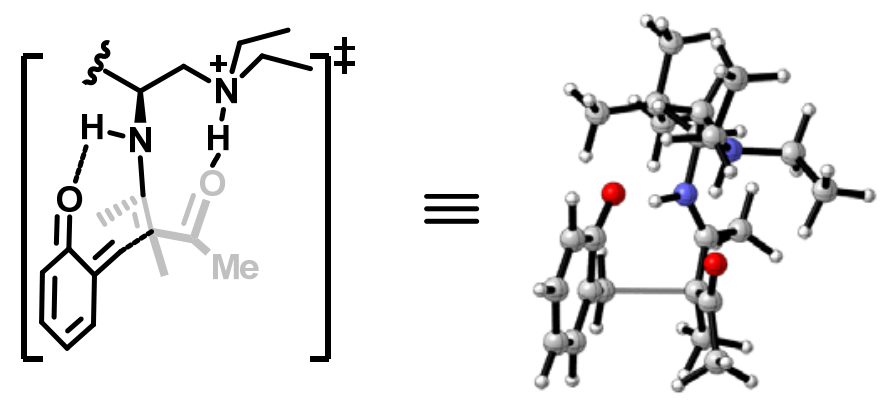

$\mathrm{E}_{\mathrm{sol}}=-1159.530717$

Zero-point correction=

Thermal correction to Energy=

0. 598243 (Hartree/Particle)

Thermal correction to Enthalpy=

0.627647

Thermal correction to Gibbs Free Energy=

Sum of electronic and zero-point Energies=

0.628591

0.541613

$-1158.519347$

$-1158.489943$

$-1158.488999$

$-1158.575977$

E (Therma1)

$\mathrm{CV}$ 


$\begin{array}{lrrr} & \text { KCal/Mol } & \text { Cal/Mol-Kelvin } & \text { Cal/Mol-Kelvin } \\ \text { Total } & 393.855 & 115.359 & 183.062 \\ \text { Electronic } & 0.000 & 0.000 & 0.000 \\ \text { Translational } & 0.889 & 2.981 & 43.660 \\ \text { Rotational } & 0.889 & 2.981 & 35.286 \\ \text { Vibrational } & 392.077 & 109.397 & 104.115\end{array}$

Standard orientation:

\begin{tabular}{|c|c|c|c|c|c|}
\hline \multirow{2}{*}{$\begin{array}{l}\text { Center } \\
\text { Number }\end{array}$} & \multirow{2}{*}{$\begin{array}{l}\text { Atomic } \\
\text { Number }\end{array}$} & \multirow{2}{*}{$\begin{array}{c}\text { Atomic } \\
\text { Type }\end{array}$} & \multicolumn{3}{|c|}{ Coordinates (Angstroms) } \\
\hline & & & $X$ & Y & Z \\
\hline 1 & 6 & 0 & 2.988064 & -0.935428 & -1.446944 \\
\hline 2 & 6 & 0 & 4. 312228 & -0.183258 & -1.638883 \\
\hline 3 & 1 & 0 & 4.925751 & -0.204931 & -0.729884 \\
\hline 4 & 1 & 0 & 4. 889725 & -0.656805 & -2.437946 \\
\hline 5 & 1 & 0 & 4. 156279 & 0.860547 & -1.929984 \\
\hline 6 & 6 & 0 & 3. 289800 & -2.425956 & -1.261810 \\
\hline 7 & 1 & 0 & 3.958759 & -2.603480 & -0.411571 \\
\hline 8 & 1 & 0 & 2. 371992 & -3.005962 & -1.117299 \\
\hline 9 & 1 & 0 & 3. 784421 & -2.814786 & -2.156783 \\
\hline 10 & 6 & 0 & 2. 107697 & -0.768100 & -2.691306 \\
\hline 11 & 1 & 0 & 1. 174941 & -1.334041 & -2.605814 \\
\hline 12 & 1 & 0 & 1. 840502 & 0.271932 & -2.898082 \\
\hline 13 & 1 & 0 & 2. 649973 & -1.148758 & -3.562403 \\
\hline 14 & 6 & 0 & 2. 286318 & -0.398891 & -0.161348 \\
\hline 15 & 1 & 0 & 2. 905925 & -0.673623 & 0.699600 \\
\hline 16 & 6 & 0 & 2. 139800 & 1. 136998 & -0.215447 \\
\hline 17 & 1 & 0 & 1. 749949 & 1. 436307 & -1.189408 \\
\hline 18 & 1 & 0 & 3. 107476 & 1. 612233 & -0.045841 \\
\hline 19 & 7 & 0 & 0.940479 & -0.939980 & 0.022691 \\
\hline 20 & 1 & 0 & 0.316346 & 1. 085094 & 0.794413 \\
\hline 21 & 7 & 0 & 1. 178770 & 1. 681380 & 0.795218 \\
\hline 22 & 6 & 0 & 0.687782 & 3. 050294 & 0.406872 \\
\hline 23 & 1 & 0 & 0.266528 & 2. 916919 & -0.592198 \\
\hline 24 & 1 & 0 & -0.136852 & 3. 279394 & 1. 084682 \\
\hline 25 & 6 & 0 & 1. 723051 & 1.583030 & 2. 190586 \\
\hline 26 & 1 & 0 & 1. 865320 & 0.513728 & 2. 374556 \\
\hline 27 & 1 & 0 & 2. 705572 & 2. 063658 & 2. 193592 \\
\hline 28 & 6 & 0 & 0.795243 & 2. 178038 & 3. 236870 \\
\hline 29 & 1 & 0 & 0.748688 & 3. 267790 & 3. 172347 \\
\hline 30 & 1 & 0 & -0.213030 & 1. 767314 & 3. 139603 \\
\hline 31 & 1 & 0 & 1. 179583 & 1. 923493 & 4. 227379 \\
\hline 32 & 6 & 0 & 1. 766703 & 4. 116560 & 0.446491 \\
\hline 33 & 1 & 0 & 2. 582909 & 3. 905867 & -0.250033 \\
\hline
\end{tabular}




\begin{tabular}{|c|c|c|c|c|c|}
\hline 34 & 1 & 0 & 1. 323840 & 5. 069816 & 0.148453 \\
\hline 35 & 1 & 0 & 2. 184126 & 4. 249128 & 1. 448783 \\
\hline 36 & 1 & 0 & 0.226754 & -0.400153 & -0.544083 \\
\hline 37 & 6 & 0 & 0.499498 & -1.888417 & 0.827498 \\
\hline 38 & 6 & 0 & -0.906711 & -1.995156 & 1. 084810 \\
\hline 39 & 6 & 0 & 1. 445982 & -2.865128 & 1. 465729 \\
\hline 40 & 1 & 0 & 2. 489548 & -2.563812 & 1. 383584 \\
\hline 41 & 1 & 0 & 1. 189535 & -3.009257 & 2. 518194 \\
\hline 42 & 1 & 0 & 1. 348234 & -3.840567 & 0.974778 \\
\hline 43 & 6 & 0 & -1.414170 & -3.335031 & 1. 580508 \\
\hline 44 & 1 & 0 & -1.001678 & -4.155551 & 0.985627 \\
\hline 45 & 1 & 0 & -1.165883 & -3.532999 & 2. 631490 \\
\hline 46 & 1 & 0 & -2.500782 & -3.398514 & 1. 491776 \\
\hline 47 & 6 & 0 & -1.586669 & -0.783937 & 1. 534838 \\
\hline 48 & 8 & 0 & -1.142726 & 0.362682 & 1. 388212 \\
\hline 49 & 6 & 0 & -2.926977 & -0.943276 & 2. 213418 \\
\hline 50 & 1 & 0 & -3.639789 & -1.436100 & 1. 543818 \\
\hline 51 & 1 & 0 & -2.845866 & -1.557059 & 3. 116110 \\
\hline 52 & 1 & 0 & -3.305915 & 0.045682 & 2. 469424 \\
\hline 53 & 6 & 0 & -3.860335 & 1. 878108 & -1.735815 \\
\hline 54 & 6 & 0 & -4.610703 & 0.722978 & -1.354049 \\
\hline 55 & 6 & 0 & -3.952306 & -0.436653 & -1.084557 \\
\hline 56 & 6 & 0 & -2.522141 & -0.521281 & -1.184880 \\
\hline 57 & 6 & 0 & -1.751052 & 0.679585 & -1.488098 \\
\hline 58 & 6 & 0 & -2.497952 & 1. 862488 & -1.815482 \\
\hline 59 & 1 & 0 & -4.396128 & 2. 794278 & -1.968631 \\
\hline 60 & 1 & 0 & -5.692453 & 0.774453 & -1.309369 \\
\hline 61 & 1 & 0 & -4.503444 & -1.340988 & -0.835363 \\
\hline 62 & 1 & 0 & -1.936053 & 2. 744557 & -2.105715 \\
\hline 63 & 8 & 0 & -0.481964 & 0.693040 & -1.452058 \\
\hline 64 & 6 & 0 & -1.866628 & -1.733442 & -1.012148 \\
\hline 65 & 1 & 0 & -2.451140 & -2.641278 & -0.881079 \\
\hline 66 & 1 & 0 & -0.870025 & -1.863870 & -1.422440 \\
\hline
\end{tabular}

(5) Int2

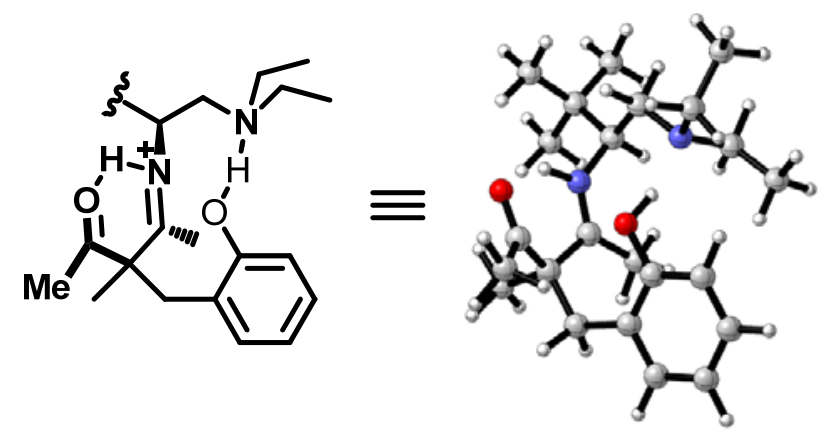


$\mathrm{E}_{\mathrm{sol}}=-1159.578363$

Zero-point correction=

0. 598645 (Hartree/Particle)

Thermal correction to Energy=

0.628732

Thermal correction to Enthalpy=

0.629676

Thermal correction to Gibbs Free Energy=

Sum of electronic and zero-point Energies=

0.538643

$-1158.565851$

$-1158.535764$

Sum of electronic and thermal Energies=

$-1158.534820$

Sum of electronic and thermal Enthalpies=

$-1158.625853$

Sum of electronic and thermal Free Energies=

\begin{tabular}{lccc} 
& E (Thermal) & CV & \multicolumn{1}{c}{ S } \\
& KCa1/Mo1 & Cal/Mo1-Kelvin & Cal/Mo1-Kelvin \\
Total & 394.535 & 116.247 & 191.595 \\
Electronic & 0.000 & 0.000 & 0.000 \\
Translational & 0.889 & 2.981 & 43.660 \\
Rotational & 0.889 & 2.981 & 35.352 \\
Vibrational & 392.758 & 110.285 & 112.582
\end{tabular}

Standard orientation:

\begin{tabular}{rrrrrr} 
Center & Atomic & Atomic & \multicolumn{3}{c}{ Coordinates (Angstroms) } \\
Number & Number & Type & X & Y & $Z$ \\
-1 & 6 & 0 & -3.674751 & -0.725129 & -0.455124 \\
1 & 6 & 0 & -4.752363 & 0.237702 & -0.973073 \\
3 & 1 & 0 & -4.488485 & 0.644653 & -1.956141 \\
4 & 1 & 0 & -5.699044 & -0.299653 & -1.080710 \\
5 & 1 & 0 & -4.931327 & 1.071653 & -0.288990 \\
6 & 6 & 0 & -3.621954 & -1.945234 & -1.384708 \\
7 & 1 & 0 & -3.339736 & -1.656677 & -2.404581 \\
8 & 1 & 0 & -2.911867 & -2.700102 & -1.030724 \\
9 & 1 & 0 & -4.606419 & -2.418860 & -1.435011 \\
10 & 6 & 0 & -4.028701 & -1.174759 & 0.968708 \\
11 & 1 & 0 & -3.291598 & -1.867543 & 1.393573 \\
12 & 1 & 0 & -4.138224 & -0.326643 & 1.651888 \\
13 & 1 & 0 & -4.986232 & -1.704011 & 0.953908 \\
14 & 6 & 0 & -2.295734 & -0.012787 & -0.509252 \\
15 & 1 & 0 & -2.134564 & 0.301996 & -1.541075 \\
16 & 6 & 0 & -2.156248 & 1.193212 & 0.433601 \\
17 & 1 & 0 & -2.174727 & 0.830703 & 1.468145 \\
18 & 1 & 0 & -3.035825 & 1.837700 & 0.295961 \\
19 & 7 & 0 & -1.237635 & -0.968702 & -0.147827 \\
20 & 7 & 0 & -0.912399 & 1.955555 & 0.248123 \\
21 & 6 & 0 & -0.649520 & 2.806722 & 1.435998 \\
22 & 1 & 0 & -0.614534 & 2.133666 & 2.299327
\end{tabular}




\begin{tabular}{|c|c|c|c|c|c|}
\hline 23 & 1 & 0 & 0.359442 & 3. 215711 & 1. 327200 \\
\hline 24 & 6 & 0 & -0.951091 & 2. 730366 & -1.007985 \\
\hline 25 & 1 & 0 & -1.088191 & 2. 021339 & -1.830845 \\
\hline 26 & 1 & 0 & -1.829361 & 3. 394024 & -1.018403 \\
\hline 27 & 6 & 0 & 0.317686 & 3. 528458 & -1.268680 \\
\hline 28 & 1 & 0 & 0.438979 & 4. 355412 & -0.563741 \\
\hline 29 & 1 & 0 & 1. 205107 & 2. 888733 & -1.208275 \\
\hline 30 & 1 & 0 & 0.276663 & 3. 958381 & -2.272447 \\
\hline 31 & 6 & 0 & -1.642205 & 3. 938915 & 1. 683305 \\
\hline 32 & 1 & 0 & -2.663296 & 3. 569708 & 1. 816854 \\
\hline 33 & 1 & 0 & -1.363746 & 4. 469846 & 2. 597078 \\
\hline 34 & 1 & 0 & -1.642806 & 4. 667665 & 0.867317 \\
\hline 35 & 1 & 0 & -1.331494 & -1.400455 & 0.789064 \\
\hline 36 & 6 & 0 & -0.113079 & -1.229622 & -0.720279 \\
\hline 37 & 6 & 0 & 0.858319 & -2.194740 & -0.047235 \\
\hline 38 & 6 & 0 & 0.233584 & -0.689312 & -2.069171 \\
\hline 39 & 1 & 0 & 0.985382 & 0.101681 & -1.953977 \\
\hline 40 & 1 & 0 & 0.689201 & -1.477598 & -2.673800 \\
\hline 41 & 1 & 0 & -0.627517 & -0.296170 & -2.606127 \\
\hline 42 & 6 & 0 & 0.413693 & -3.622314 & -0.467956 \\
\hline 43 & 1 & 0 & -0.584317 & -3.846708 & -0.082907 \\
\hline 44 & 1 & 0 & 0.408952 & -3.724924 & -1.557673 \\
\hline 45 & 1 & 0 & 1. 114925 & -4.359720 & -0.065887 \\
\hline 46 & 6 & 0 & 0.733629 & -2.162068 & 1. 498359 \\
\hline 47 & 8 & 0 & -0.368141 & -2.123963 & 2. 016998 \\
\hline 48 & 6 & 0 & 4. 347549 & 1. 830965 & -0.253268 \\
\hline 49 & 6 & 0 & 4. 923553 & 0.748196 & -0.915869 \\
\hline 50 & 6 & 0 & 4. 240553 & -0.459067 & -0.979130 \\
\hline 51 & 6 & 0 & 2. 977418 & -0.621103 & -0.397595 \\
\hline 52 & 6 & 0 & 2. 414471 & 0.477278 & 0.264489 \\
\hline 53 & 6 & 0 & 3. 100728 & 1. 691404 & 0.341665 \\
\hline 54 & 1 & 0 & 4. 874040 & 2. 777538 & -0.188330 \\
\hline 55 & 1 & 0 & 5.900379 & 0.844262 & -1.377209 \\
\hline 56 & 1 & 0 & 4. 691470 & -1.303842 & -1.495363 \\
\hline 57 & 1 & 0 & 2. 647533 & 2. 510777 & 0.892758 \\
\hline 58 & 8 & 0 & 1. 192145 & 0.328623 & 0.866304 \\
\hline 59 & 6 & 0 & 2. 309862 & -1.981420 & -0.531531 \\
\hline 60 & 1 & 0 & 2. 364357 & -2.284926 & -1.583802 \\
\hline 61 & 1 & 0 & 2. 921591 & -2.724666 & -0.006944 \\
\hline 62 & 6 & 0 & 1. 969435 & -2.312033 & 2. 339155 \\
\hline 63 & 1 & 0 & 2. 708706 & -1.544774 & 2. 093330 \\
\hline 64 & 1 & 0 & 1. 680492 & -2.236867 & 3. 387004 \\
\hline 65 & 1 & 0 & 2. 428997 & -3.291944 & 2. 164422 \\
\hline 66 & 1 & 0 & 0.516309 & 1. 003608 & 0.5501 \\
\hline
\end{tabular}


(6) $\mathrm{TS}_{2}$

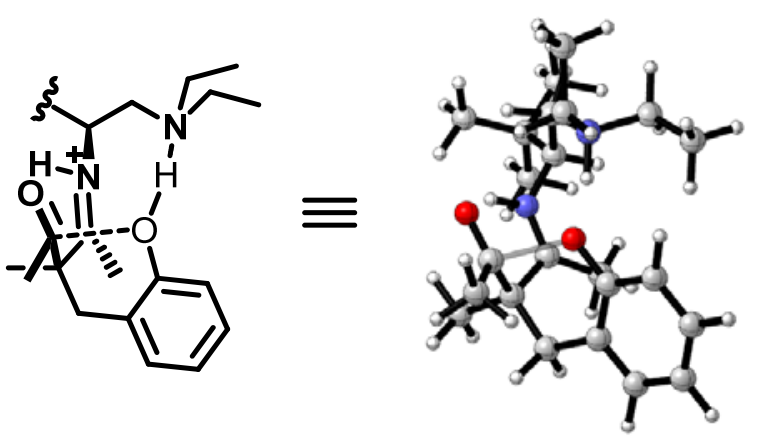

$\mathrm{E}_{\mathrm{sol}}=-1159.565403$

Zero-point correction=

0.598617 (Hartree/Particle)

Thermal correction to Energy=

0. 627281

Thermal correction to Enthalpy=

0.628226

Thermal correction to Gibbs Free Energy=

0.542271

Sum of electronic and zero-point Energies=

$-1158.548321$

Sum of electronic and thermal Energies=

$-1158.519657$

Sum of electronic and thermal Enthalpies=

$-1158.518712$

Sum of electronic and thermal Free Energies=

$-1158.604667$

\section{E (Therma1) \\ KCa1/Mol}

Total

393. 625

Electronic

Translational

Rotational

Vibrational

Standard orientation:
0. 000
0.889
0.889

391.848
CV

Cal/Mo1-Kelvin

113. 458

0.000

2. 981

2. 981

107. 496
$\mathrm{S}$

Cal/Mo1-Kelvin

180. 906

0. 000

43. 660

35. 263

101.982

\begin{tabular}{|c|c|c|c|c|c|}
\hline \multirow{2}{*}{$\begin{array}{l}\text { Center } \\
\text { Number }\end{array}$} & \multirow{2}{*}{$\begin{array}{l}\text { Atomic } \\
\text { Number }\end{array}$} & \multirow{2}{*}{$\begin{array}{l}\text { Atomic } \\
\text { Type }\end{array}$} & \multicolumn{3}{|c|}{ Coordinates (Angstroms) } \\
\hline & & & X & Y & Z \\
\hline 1 & 6 & 0 & -3.640444 & -0.867865 & -0.726211 \\
\hline 2 & 6 & 0 & -4.632901 & 0.073750 & -1.420304 \\
\hline 3 & 1 & 0 & -4.234469 & 0.448608 & -2.370624 \\
\hline 4 & 1 & 0 & -5.556348 & -0.468485 & -1.642636 \\
\hline 5 & 1 & 0 & -4.908057 & 0.930046 & -0.796772 \\
\hline 6 & 6 & 0 & -3.460454 & -2.121987 & -1.590596 \\
\hline 7 & 1 & 0 & -3.094587 & -1.867056 & -2.592625 \\
\hline 8 & 1 & 0 & -2.763841 & -2.833295 & -1.134397 \\
\hline 9 & 1 & 0 & -4.421156 & -2.630619 & -1.710874 \\
\hline 10 & 6 & 0 & -4.170144 & -1.265987 & 0.656287 \\
\hline 11 & 1 & 0 & -3.474086 & -1.924377 & 1. 188733 \\
\hline
\end{tabular}




\begin{tabular}{|c|c|c|c|c|c|}
\hline 12 & 1 & 0 & -4.376835 & -0.394849 & 1. 286987 \\
\hline 13 & 1 & 0 & -5.111432 & -1.811694 & 0.543174 \\
\hline 14 & 6 & 0 & -2.262831 & -0.164167 & -0.618746 \\
\hline 15 & 1 & 0 & -1.944774 & 0.103024 & -1.631530 \\
\hline 16 & 6 & 0 & -2.265082 & 1. 084737 & 0.275508 \\
\hline 17 & 1 & 0 & -2.391905 & 0.789024 & 1. 320983 \\
\hline 18 & 1 & 0 & -3.084226 & 1. 754815 & 0.004980 \\
\hline 19 & 7 & 0 & -1.257452 & -1.041925 & -0.018009 \\
\hline 20 & 7 & 0 & -0.986523 & 1. 862123 & 0.183019 \\
\hline 21 & 6 & 0 & -0.673103 & 2. 574979 & 1. 476904 \\
\hline 22 & 1 & 0 & -0.608810 & 1. 776972 & 2. 222697 \\
\hline 23 & 1 & 0 & 0.334752 & 2. 977005 & 1. 359246 \\
\hline 24 & 6 & 0 & -1.014988 & 2. 759183 & -1.018863 \\
\hline 25 & 1 & 0 & -1.325487 & 2. 137201 & -1.863020 \\
\hline 26 & 1 & 0 & -1.801415 & 3. 500145 & -0.850628 \\
\hline 27 & 6 & 0 & 0.322093 & 3. 409680 & -1.315842 \\
\hline 28 & 1 & 0 & 0.629944 & 4. 100690 & -0.526865 \\
\hline 29 & 1 & 0 & 1. 105583 & 2. 659942 & -1.455195 \\
\hline 30 & 1 & 0 & 0.232049 & 3. 984886 & -2.240456 \\
\hline 31 & 6 & 0 & -1.678227 & 3. 647950 & 1. 849780 \\
\hline 32 & 1 & 0 & -2.696291 & 3. 255543 & 1. 930359 \\
\hline 33 & 1 & 0 & -1.406908 & 4. 047778 & 2. 829664 \\
\hline 34 & 1 & 0 & -1.676962 & 4. 485001 & 1. 146284 \\
\hline 35 & 1 & 0 & -1.224337 & -1.026769 & 1. 046966 \\
\hline 36 & 6 & 0 & -0.125404 & -1.451247 & -0.490821 \\
\hline 37 & 6 & 0 & 0.850724 & -2.009929 & 0.532048 \\
\hline 38 & 6 & 0 & 0.204182 & -1.431646 & -1.941680 \\
\hline 39 & 1 & 0 & 1. 088223 & -0.803443 & -2.103150 \\
\hline 40 & 1 & 0 & 0.480313 & -2.447032 & -2.247276 \\
\hline 41 & 1 & 0 & -0.622085 & -1.100188 & -2.568561 \\
\hline 42 & 6 & 0 & 0.347683 & -3.395250 & 0.990139 \\
\hline 43 & 1 & 0 & -0.643396 & -3.328408 & 1. 447124 \\
\hline 44 & 1 & 0 & 0.303686 & -4.091873 & 0.146265 \\
\hline 45 & 1 & 0 & 1. 038112 & -3.810341 & 1. 729887 \\
\hline 46 & 6 & 0 & 0.825597 & -1.041980 & 1. 760913 \\
\hline 47 & 8 & 0 & -0.280169 & -0.741465 & 2. 259163 \\
\hline 48 & 6 & 0 & 4. 401436 & 1. 487038 & -0.999342 \\
\hline 49 & 6 & 0 & 4. 909619 & 0.250809 & -1.394295 \\
\hline 50 & 6 & 0 & 4. 191604 & -0.901912 & -1.097525 \\
\hline 51 & 6 & 0 & 2. 961735 & -0.847503 & -0.438949 \\
\hline 52 & 6 & 0 & 2. 425599 & 0.408100 & -0.080402 \\
\hline 53 & 6 & 0 & 3. 180407 & 1. 563417 & -0.341916 \\
\hline 54 & 1 & 0 & 4. 964603 & 2. 394835 & -1.193071 \\
\hline 55 & 1 & 0 & 5. 862671 & 0.183902 & -1.90734 \\
\hline
\end{tabular}




$\begin{array}{rrrrrr}56 & 1 & 0 & 4.596749 & -1.874105 & -1.371869 \\ 57 & 1 & 0 & 2.796402 & 2.517342 & 0.008042 \\ 58 & 8 & 0 & 1.231594 & 0.484945 & 0.525843 \\ 59 & 6 & 0 & 2.269262 & -2.139581 & -0.050168 \\ 60 & 1 & 0 & 2.247553 & -2.830241 & -0.903176 \\ 61 & 1 & 0 & 2.872345 & -2.653937 & 0.709232 \\ 62 & 6 & 0 & 2.043267 & -0.976897 & 2.645832 \\ 63 & 1 & 0 & 2.970340 & -0.784941 & 2.106779 \\ 64 & 1 & 0 & 1.873123 & -0.195357 & 3.387027 \\ 65 & 1 & 0 & 2.133584 & -1.934491 & 3.173428 \\ 66 & 1 & 0 & -0.123980 & 1.213352 & 0.116595\end{array}$

(7) Int3

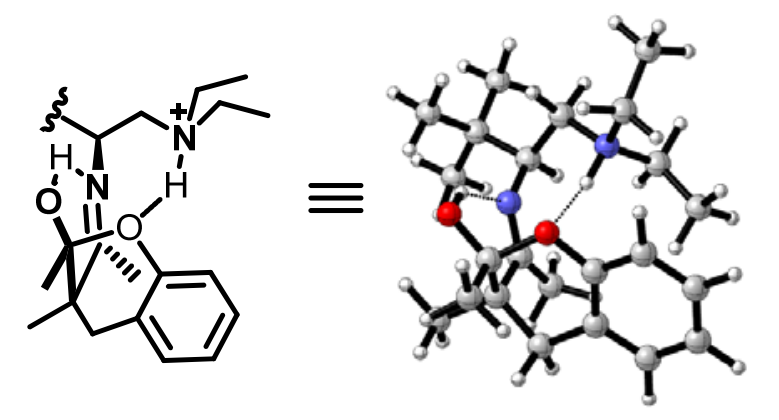

$\mathrm{E}_{\mathrm{sol}}=-1159.596873$

Zero-point correction=

0. 604277 (Hartree/Particle)

Thermal correction to Energy=

0.631697

Thermal correction to Enthalpy=

0.632641

Thermal correction to Gibbs Free Energy=

Sum of electronic and zero-point Energies=

0.550529

Sum of electronic and thermal Energies=

$-1158.577240$

$-1158.549820$

Sum of electronic and thermal Enthalpies=

$-1158.548875$

Sum of electronic and thermal Free Energies=

$-1158.630988$

$\begin{array}{lccc} & \text { E (Thermal) } & \text { CV } & \text { S } \\ & \text { KCal/Mol } & \text { Cal/Mol-Kelvin } & \text { Cal/Mol-Kelvin } \\ \text { Total } & 396.396 & 111.759 & 172.820 \\ \text { Electronic } & 0.000 & 0.000 & 0.000 \\ \text { Translational } & 0.889 & 2.981 & 43.660 \\ \text { Rotational } & 0.889 & 2.981 & 35.145 \\ \text { Vibrational } & 394.618 & 105.797 & 94.015\end{array}$

Standard orientation:

\begin{tabular}{|c|c|c|c|c|c|}
\hline \multirow{2}{*}{$\begin{array}{l}\text { Center } \\
\text { Number }\end{array}$} & \multirow{2}{*}{$\begin{array}{l}\text { Atomic } \\
\text { Number }\end{array}$} & \multirow{2}{*}{$\begin{array}{l}\text { Atomic } \\
\text { Type }\end{array}$} & \multicolumn{3}{|c|}{ Coordinates (Angstroms) } \\
\hline & & & $X$ & Y & Z \\
\hline \multirow[t]{2}{*}{1} & 6 & 0 & -3.725940 & -0.287775 & -0.271855 \\
\hline & & & S42 & & \\
\hline
\end{tabular}




\begin{tabular}{|c|c|c|c|c|c|}
\hline 2 & 6 & 0 & -4.757159 & 0.815341 & -0.538045 \\
\hline 3 & 1 & 0 & -4.603438 & 1. 283418 & -1.517972 \\
\hline 4 & 1 & 0 & -5.765090 & 0.390296 & -0.530206 \\
\hline 5 & 1 & 0 & -4.731106 & 1. 596494 & 0.229816 \\
\hline 6 & 6 & 0 & -3.944965 & -1.436990 & -1.263654 \\
\hline 7 & 1 & 0 & -3.896350 & -1.083812 & -2.300670 \\
\hline 8 & 1 & 0 & -3.197550 & -2.226982 & -1.128393 \\
\hline 9 & 1 & 0 & -4.931932 & -1.883505 & -1.110742 \\
\hline 10 & 6 & 0 & -3.888369 & -0.822874 & 1. 155603 \\
\hline 11 & 1 & 0 & -3.214774 & -1.662603 & 1. 351119 \\
\hline 12 & 1 & 0 & -3.715094 & -0.054926 & 1. 917529 \\
\hline 13 & 1 & 0 & -4.913152 & -1.181606 & 1. 292031 \\
\hline 14 & 6 & 0 & -2.297344 & 0.272706 & -0.526366 \\
\hline 15 & 1 & 0 & -2.243440 & 0.505370 & -1.599316 \\
\hline 16 & 6 & 0 & -2.004996 & 1. 552079 & 0.273461 \\
\hline 17 & 1 & 0 & -2.193311 & 1. 380905 & 1. 336471 \\
\hline 18 & 1 & 0 & -2.600616 & 2. 399454 & -0.071663 \\
\hline 19 & 7 & 0 & -1.288485 & -0.663073 & -0.083367 \\
\hline 20 & 7 & 0 & -0.553885 & 1. 937547 & 0.149742 \\
\hline 21 & 6 & 0 & -0.079321 & 2. 775304 & 1. 311463 \\
\hline 22 & 1 & 0 & -0.152602 & 2. 119433 & 2. 183337 \\
\hline 23 & 1 & 0 & 0.979982 & 2. 966397 & 1. 132102 \\
\hline 24 & 6 & 0 & -0.277669 & 2. 549587 & -1.199787 \\
\hline 25 & 1 & 0 & -0.793423 & 1. 922084 & -1.929777 \\
\hline 26 & 1 & 0 & -0.767251 & 3. 526458 & -1.198700 \\
\hline 27 & 6 & 0 & 1. 196064 & 2. 646189 & -1.546185 \\
\hline 28 & 1 & 0 & 1.752185 & 3. 297075 & -0.868383 \\
\hline 29 & 1 & 0 & 1. 674615 & 1. 661528 & -1.552356 \\
\hline 30 & 1 & 0 & 1. 278968 & 3. 069558 & -2.550554 \\
\hline 31 & 6 & 0 & -0.858342 & 4. 063682 & 1. 500912 \\
\hline 32 & 1 & 0 & -1.916320 & 3. 882407 & 1. 705901 \\
\hline 33 & 1 & 0 & -0.443615 & 4. 586229 & 2. 366171 \\
\hline 34 & 1 & 0 & -0.771337 & 4. 737260 & 0.644426 \\
\hline 35 & 1 & 0 & -0.984798 & -1.263812 & 1. 632971 \\
\hline 36 & 6 & 0 & -0.435450 & -1.260651 & -0.821054 \\
\hline 37 & 6 & 0 & 0.595692 & -2.156640 & -0.111849 \\
\hline 38 & 6 & 0 & -0.388299 & -1.197597 & -2.330351 \\
\hline 39 & 1 & 0 & 0.451891 & -0.571385 & -2.655748 \\
\hline 40 & 1 & 0 & -0.220378 & -2.195749 & -2.744075 \\
\hline 41 & 1 & 0 & -1.310705 & -0.810712 & -2.762732 \\
\hline 42 & 6 & 0 & 0.030506 & -3.585463 & -0.131793 \\
\hline 43 & 1 & 0 & -0.873429 & -3.643635 & 0.479899 \\
\hline 44 & 1 & 0 & -0.213415 & -3.897949 & -1.152425 \\
\hline 45 & 1 & 0 & 0.761138 & -4.296680 & 0.2618 \\
\hline
\end{tabular}




$\begin{array}{rrrrrr}46 & 6 & 0 & 0.835373 & -1.684764 & 1.363148 \\ 47 & 8 & 0 & -0.292187 & -1.726823 & 2.146779 \\ 48 & 6 & 0 & 4.120344 & 1.542607 & 0.020803 \\ 49 & 6 & 0 & 4.428345 & 0.809516 & -1.124946 \\ 50 & 6 & 0 & 3.710134 & -0.346684 & -1.414546 \\ 51 & 6 & 0 & 2.661188 & -0.779305 & -0.598131 \\ 52 & 6 & 0 & 2.331403 & 0.004817 & 0.516159 \\ 53 & 6 & 0 & 3.074669 & 1.140198 & 0.843024 \\ 54 & 1 & 0 & 4.702725 & 2.420899 & 0.280598 \\ 55 & 1 & 0 & 5.241958 & 1.119598 & -1.771576 \\ 56 & 1 & 0 & 3.974703 & -0.943296 & -2.284395 \\ 57 & 1 & 0 & 2.835273 & 1.670475 & 1.759999 \\ 58 & 8 & 0 & 1.246584 & -0.278916 & 1.307714 \\ 59 & 6 & 0 & 1.952610 & -2.092300 & -0.836468 \\ 60 & 1 & 0 & 1.843372 & -2.283881 & -1.908069 \\ 61 & 1 & 0 & 2.578174 & -2.910300 & -0.457912 \\ 62 & 6 & 0 & 1.913916 & -2.458865 & 2.105470 \\ 63 & 1 & 0 & 2.902028 & -2.331957 & 1.659594 \\ 64 & 1 & 0 & 1.936433 & -2.074432 & 3.126918 \\ 65 & 1 & 0 & 1.663381 & -3.520675 & 2.144573 \\ 66 & 1 & 0 & -0.016621 & 1.054447 & 0.239756 \\ --------------------------------------------------\end{array}$

(8) Intza

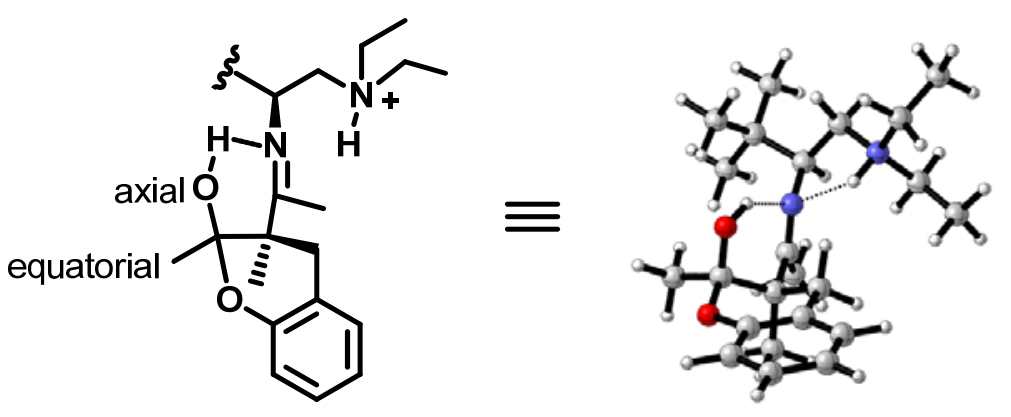

$\mathrm{E}_{\mathrm{sol}}=-1159.591199$

Zero-point correction=

0.603171 (Hartree/Particle)

Thermal correction to Energy=

0.631698

Thermal correction to Enthalpy=

0.632642

Thermal correction to Gibbs Free Energy=

0.546635

Sum of electronic and zero-point Energies=

$-1158.558725$

$-1158.530198$

Sum of electronic and thermal Energies=

$-1158.529254$

Sum of electronic and thermal Enthalpies=

$-1158.615261$

Sum of electronic and thermal Free Energies=

E (Therma1)

CV

S

Total

KCa1/Mol

396. 397

Cal/Mo1-Kelvin

Cal/Mo1-Kelvin

114. 154

181. 018 


$\begin{array}{lrrr}\text { Electronic } & 0.000 & 0.000 & 0.000 \\ \text { Translational } & 0.889 & 2.981 & 43.660 \\ \text { Rotational } & 0.889 & 2.981 & 35.609 \\ \text { Vibrational } & 394.619 & 108.192 & 101.749\end{array}$

Standard orientation:

\begin{tabular}{|c|c|c|c|c|c|}
\hline \multirow{2}{*}{$\begin{array}{l}\text { Center } \\
\text { Number }\end{array}$} & \multirow{2}{*}{$\begin{array}{l}\text { Atomic } \\
\text { Number }\end{array}$} & \multirow{2}{*}{$\begin{array}{c}\text { Atomic } \\
\text { Type }\end{array}$} & \multicolumn{3}{|c|}{ Coordinates (Angstroms) } \\
\hline & & & $X$ & Y & Z \\
\hline 1 & 6 & 0 & 3. 174454 & -1.746436 & -0.496809 \\
\hline 2 & 6 & 0 & 4. 681493 & -1.519067 & -0.682532 \\
\hline 3 & 1 & 0 & 5. 168786 & -1.255795 & 0.264131 \\
\hline 4 & 1 & 0 & 5. 149198 & -2.437310 & -1.049199 \\
\hline 5 & 1 & 0 & 4. 898622 & -0.735399 & -1.416674 \\
\hline 6 & 6 & 0 & 2.974385 & -2.982455 & 0.385850 \\
\hline 7 & 1 & 0 & 3. 474383 & -2.871172 & 1. 354835 \\
\hline 8 & 1 & 0 & 1. 914011 & -3.187722 & 0.562405 \\
\hline 9 & 1 & 0 & 3. 399046 & -3.861386 & -0.108099 \\
\hline 10 & 6 & 0 & 2.511073 & -1.997557 & -1.856671 \\
\hline 11 & 1 & 0 & 1. 444713 & -2.222176 & -1.757255 \\
\hline 12 & 1 & 0 & 2. 615969 & -1.152667 & -2.546790 \\
\hline 13 & 1 & 0 & 2. 988203 & -2.857615 & -2.336203 \\
\hline 14 & 6 & 0 & 2. 557766 & -0.507487 & 0.224576 \\
\hline 15 & 1 & 0 & 2. 962601 & -0.494749 & 1. 246319 \\
\hline 16 & 6 & 0 & 2. 984852 & 0.798553 & -0.480196 \\
\hline 17 & 1 & 0 & 2. 943112 & 0.677581 & -1.565496 \\
\hline 18 & 1 & 0 & 3. 992261 & 1. 104825 & -0.193594 \\
\hline 19 & 7 & 0 & 1. 104796 & -0.516421 & 0.197498 \\
\hline 20 & 1 & 0 & 1. 110878 & 1. 472696 & -0.222198 \\
\hline 21 & 7 & 0 & 2. 041616 & 1. 920282 & -0.151322 \\
\hline 22 & 6 & 0 & 2. 087080 & 3. 038214 & -1.161540 \\
\hline 23 & 1 & 0 & 1. 924496 & 2.567025 & -2.133633 \\
\hline 24 & 1 & 0 & 1. 229335 & 3. 681022 & -0.955415 \\
\hline 25 & 6 & 0 & 2. 181614 & 2. 371648 & 1. 277241 \\
\hline 26 & 1 & 0 & 2. 009703 & 1. 480473 & 1. 886584 \\
\hline 27 & 1 & 0 & 3. 219822 & 2. 684372 & 1. 413879 \\
\hline 28 & 6 & 0 & 1. 198636 & 3. 469829 & 1. 643814 \\
\hline 29 & 1 & 0 & 1. 436721 & 4. 420684 & 1. 161494 \\
\hline 30 & 1 & 0 & 0.171508 & 3. 189376 & 1. 387483 \\
\hline 31 & 1 & 0 & 1. 239539 & 3. 629300 & 2. 723603 \\
\hline 32 & 6 & 0 & 3. 389870 & 3. 815855 & -1.129752 \\
\hline 33 & 1 & 0 & 4. 254634 & 3. 181162 & -1.339817 \\
\hline 34 & 1 & 0 & 3. 351247 & 4. 584305 & -1.905198 \\
\hline 35 & 1 & 0 & 3. 543414 & 4. 322836 & -0.173443 \\
\hline
\end{tabular}




\begin{tabular}{|c|c|c|c|c|c|}
\hline 36 & 1 & 0 & -0.095859 & -0.773865 & -1.295864 \\
\hline 37 & 6 & 0 & 0.344913 & -0.944329 & 1. 139686 \\
\hline 38 & 6 & 0 & -1.169983 & -0.832375 & 0.902064 \\
\hline 39 & 6 & 0 & 0.818730 & -1.533909 & 2. 442467 \\
\hline 40 & 1 & 0 & 1. 899611 & -1.493206 & 2. 567458 \\
\hline 41 & 1 & 0 & 0.339468 & -1.026673 & 3. 284061 \\
\hline 42 & 1 & 0 & 0.515145 & -2.585154 & 2. 498681 \\
\hline 43 & 6 & 0 & -1.994697 & -1.336586 & 2. 094054 \\
\hline 44 & 1 & 0 & -1.750460 & -2.365885 & 2. 366592 \\
\hline 45 & 1 & 0 & -1.840265 & -0.704639 & 2. 974047 \\
\hline 46 & 1 & 0 & -3.057657 & -1.304076 & 1. 850401 \\
\hline 47 & 6 & 0 & -1.576041 & -1.628292 & -0.397925 \\
\hline 48 & 8 & 0 & -1.002043 & -1.041089 & -1.535430 \\
\hline 49 & 6 & 0 & -5.530821 & 0.984561 & -0.936176 \\
\hline 50 & 6 & 0 & -4.928265 & 2. 091667 & -0.337197 \\
\hline 51 & 6 & 0 & -3.643214 & 1. 974671 & 0.175179 \\
\hline 52 & 6 & 0 & -2.938959 & 0.772048 & 0.095798 \\
\hline 53 & 6 & 0 & -3.559072 & -0.322253 & -0.509241 \\
\hline 54 & 6 & 0 & -4.851362 & -0.222291 & -1.020742 \\
\hline 55 & 1 & 0 & -6.535836 & 1. 062431 & -1.337844 \\
\hline 56 & 1 & 0 & -5.460465 & 3. 034051 & -0.265683 \\
\hline 57 & 1 & 0 & -3.170062 & 2. 828945 & 0.655571 \\
\hline 58 & 1 & 0 & -5.296756 & -1.099778 & -1.476851 \\
\hline 59 & 8 & 0 & -2.969607 & -1.559492 & -0.565834 \\
\hline 60 & 6 & 0 & -1.534844 & 0.642688 & 0.623203 \\
\hline 61 & 1 & 0 & -0.843879 & 1. 044773 & -0.130274 \\
\hline 62 & 1 & 0 & -1.415939 & 1. 231567 & 1. 543094 \\
\hline 63 & 6 & 0 & -1.253788 & -3.112709 & -0.333802 \\
\hline 64 & 1 & 0 & -1.568959 & -3.563608 & -1.276940 \\
\hline 65 & 1 & 0 & -0.175289 & -3.264735 & -0.221614 \\
\hline 66 & 1 & 0 & -1.779611 & -3.606113 & 0.486237 \\
\hline
\end{tabular}

(9) Int4

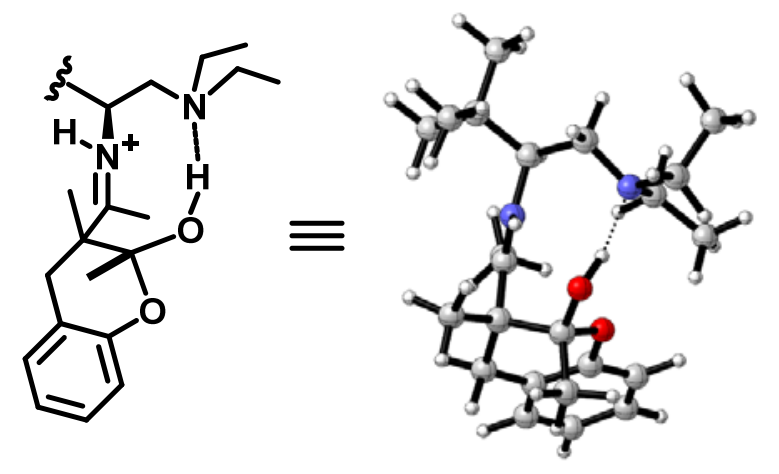

$\mathrm{E}_{\mathrm{sol}}=-1159.586123$

Zero-point correction=

0. 602907 (Hartree/Particle) 
Thermal correction to Energy=

0.631130

Thermal correction to Enthalpy=

0.632074

Thermal correction to Gibbs Free Energy=

0.547756

Sum of electronic and zero-point Energies=

$-1158.573145$

Sum of electronic and thermal Energies=

$-1158.544922$

Sum of electronic and thermal Enthalpies=

$-1158.543978$

Sum of electronic and thermal Free Energies=

$-1158.628296$

\section{E (Therma1)}

KCa1/Mol

Total

396. 040

Electronic

Translational

Rotational

Vibrational

Standard orientation:

$\begin{array}{cc}\text { CV } & \text { S } \\ \text { Ca1/Mo1-Ke1vin } & \text { Ca1/Mo1-Ke1vin } \\ \text { 113. } 563 & 177.462 \\ 0.000 & 0.000 \\ 2.981 & 43.660 \\ 2.981 & 35.348 \\ 107.601 & 98.454\end{array}$

\begin{tabular}{lllll} 
Center & Atomic & Atomic & \multicolumn{2}{c}{ Coordinates (Angstroms) } \\
Number & Number & Type & X & Y
\end{tabular}

$\begin{array}{rrrrrr}1 & 6 & 0 & -3.322097 & -1.703218 & -0.707358 \\ 2 & 6 & 0 & -4.555802 & -1.190576 & -1.461316 \\ 3 & 1 & 0 & -4.284771 & -0.774450 & -2.438686 \\ 4 & 1 & 0 & -5.248789 & -2.019026 & -1.634595 \\ 5 & 1 & 0 & -5.095714 & -0.424665 & -0.896726 \\ 6 & 6 & 0 & -2.743146 & -2.906715 & -1.461434 \\ 7 & 1 & 0 & -2.503555 & -2.649764 & -2.499954 \\ 8 & 1 & 0 & -1.839298 & -3.297740 & -0.978482 \\ 9 & 1 & 0 & -3.474598 & -3.719727 & -1.484927 \\ 10 & 6 & 0 & -3.723141 & -2.147765 & 0.705543 \\ 11 & 1 & 0 & -2.881879 & -2.593157 & 1.250125 \\ 12 & 1 & 0 & -4.136511 & -1.330518 & 1.305388 \\ 13 & 1 & 0 & -4.498448 & -2.916197 & 0.634509 \\ 14 & 6 & 0 & -2.250591 & -0.578563 & -0.673313 \\ 15 & 1 & 0 & -1.865277 & -0.434933 & -1.685583 \\ 16 & 6 & 0 & -2.754013 & 0.772245 & -0.132615 \\ 17 & 1 & 0 & -3.301667 & 0.605785 & 0.803224 \\ 18 & 1 & 0 & -3.470946 & 1.168621 & -0.865427 \\ 19 & 7 & 0 & -1.119469 & -0.975363 & 0.184618 \\ 20 & 7 & 0 & -1.682271 & 1.731843 & 0.146586 \\ 21 & 6 & 0 & -2.188737 & 2.848073 & 0.976134 \\ 22 & 1 & 0 & -2.582252 & 2.400013 & 1.895566 \\ 23 & 1 & 0 & -1.326077 & 3.447947 & 1.276175 \\ 24 & 6 & 0 & -1.014372 & 2.164674 & -1.095686\end{array}$




\begin{tabular}{|c|c|c|c|c|c|}
\hline 25 & 1 & 0 & -0.474289 & 1. 294811 & -1.490984 \\
\hline 26 & 1 & 0 & -1.761434 & 2. 449749 & -1.854912 \\
\hline 27 & 6 & 0 & -0.015305 & 3. 295327 & -0.889971 \\
\hline 28 & 1 & 0 & -0.508192 & 4. 248695 & -0.684245 \\
\hline 29 & 1 & 0 & 0.666138 & 3. 061337 & -0.067802 \\
\hline 30 & 1 & 0 & 0.575479 & 3. 420857 & -1.801419 \\
\hline 31 & 6 & 0 & -3.249544 & 3. 732647 & 0.325466 \\
\hline 32 & 1 & 0 & -4.165382 & 3. 179330 & 0.097060 \\
\hline 33 & 1 & 0 & -3.519274 & 4. 542170 & 1. 008542 \\
\hline 34 & 1 & 0 & -2.886540 & 4. 189340 & -0.600618 \\
\hline 35 & 1 & 0 & -1.290495 & -0.826107 & 1. 181426 \\
\hline 36 & 6 & 0 & 0.100758 & -1.282452 & -0.112481 \\
\hline 37 & 6 & 0 & 1. 063980 & -1.389479 & 1. 062214 \\
\hline 38 & 6 & 0 & 0.519271 & -1.451820 & -1.535474 \\
\hline 39 & 1 & 0 & 1. 028503 & -0.538469 & -1.867425 \\
\hline 40 & 1 & 0 & 1. 246529 & -2.260567 & -1.623840 \\
\hline 41 & 1 & 0 & -0.323443 & -1.660565 & -2.192659 \\
\hline 42 & 6 & 0 & 0.481056 & -2.317611 & 2. 141508 \\
\hline 43 & 1 & 0 & -0.373030 & -1.869454 & 2. 658386 \\
\hline 44 & 1 & 0 & 0.174673 & -3.276338 & 1. 711040 \\
\hline 45 & 1 & 0 & 1.241530 & -2.522108 & 2. 898068 \\
\hline 46 & 6 & 0 & 1. 214911 & 0.091721 & 1. 613992 \\
\hline 47 & 8 & 0 & -0.001362 & 0.603762 & 2. 030489 \\
\hline 48 & 6 & 0 & 4. 525967 & 1. 124169 & -1.681966 \\
\hline 49 & 6 & 0 & 4. 983113 & -0.193849 & -1.689114 \\
\hline 50 & 6 & 0 & 4. 318536 & -1.155247 & -0.938480 \\
\hline 51 & 6 & 0 & 3. 198795 & -0.827565 & -0.169825 \\
\hline 52 & 6 & 0 & 2. 766618 & 0.501642 & -0.168958 \\
\hline 53 & 6 & 0 & 3. 418583 & 1. 476027 & -0.921182 \\
\hline 54 & 1 & 0 & 5. 038340 & 1. 883106 & -2.263942 \\
\hline 55 & 1 & 0 & 5. 851554 & -0.468662 & -2.277518 \\
\hline 56 & 1 & 0 & 4. 666262 & -2.185595 & -0.944865 \\
\hline 57 & 1 & 0 & 3. 050865 & 2. 496309 & -0.887650 \\
\hline 58 & 8 & 0 & 1. 637869 & 0.900977 & 0.516868 \\
\hline 59 & 6 & 0 & 2. 457870 & -1.869486 & 0.633521 \\
\hline 60 & 1 & 0 & 2. 390737 & -2.814373 & 0.081479 \\
\hline 61 & 1 & 0 & 3. 018698 & -2.108194 & 1. 544361 \\
\hline 62 & 6 & 0 & 2. 195573 & 0.198440 & 2. 771022 \\
\hline 63 & 1 & 0 & 3. 204555 & -0.108597 & 2. 489934 \\
\hline 64 & 1 & 0 & 2. 217236 & 1. 248064 & 3. 070258 \\
\hline 65 & 1 & 0 & 1. 852594 & -0.393672 & 3. 622143 \\
\hline 66 & 1 & 0 & -0.461406 & 1. 079768 & 1. 275913 \\
\hline
\end{tabular}

(10) Int4a 

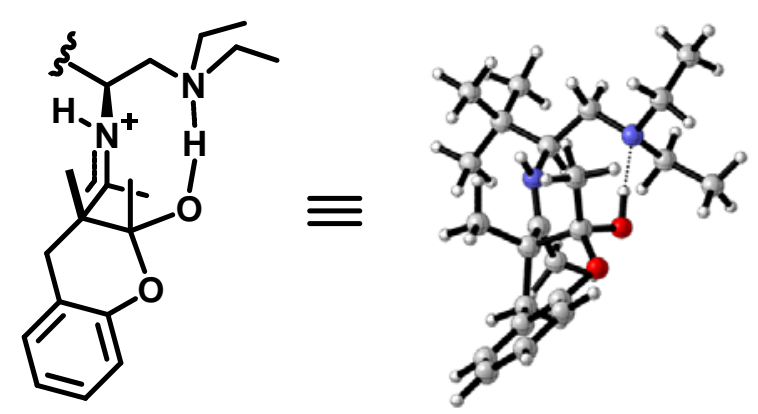

$\mathrm{E}_{\mathrm{sol}}=-1159.575432$

Zero-point correction=

0.601950 (Hartree/Particle)

Thermal correction to Energy=

0.630527

Thermal correction to Enthalpy=

0.631471

Thermal correction to Gibbs Free Energy=

Sum of electronic and zero-point Energies=

0.545012

$-1158.554519$

$-1158.525942$

$-1158.524998$

Sum of electronic and thermal Enthalpies=

$-1158.611457$

Sum of electronic and thermal Free Energies=

E (Therma1)

CV

$\mathrm{S}$

KCa1/Mol

Total

Electronic

Translational

Rotational

Vibrational
395. 662

0. 000

0.889

0.889

393. 884
Cal/Mo1-Kelvin

114. 155

0. 000

2. 981

2. 981

108. 194
Cal/Mo1-Kelvin

181.968

0.000

43. 660

35. 632

102.676

Standard orientation:

\begin{tabular}{rrrrrr} 
Center & Atomic & Atomic & \multicolumn{3}{c}{ Coordinates (Angstroms) } \\
Number & Number & Type & X & Y & $Z$ \\
$---1---1$ & & & \\
1 & 6 & 0 & 3.199336 & -2.271670 & -0.319801 \\
2 & 6 & 0 & 4.721826 & -2.138431 & -0.185634 \\
3 & 1 & 0 & 5.008172 & -1.800885 & 0.817216 \\
4 & 1 & 0 & 5.189121 & -3.112978 & -0.353267 \\
5 & 1 & 0 & 5.140750 & -1.442730 & -0.918405 \\
6 & 6 & 0 & 2.718273 & -3.424506 & 0.570821 \\
7 & 1 & 0 & 3.017355 & -3.271520 & 1.614348 \\
8 & 1 & 0 & 1.628943 & -3.542757 & 0.533882 \\
9 & 1 & 0 & 3.159966 & -4.367213 & 0.234999 \\
10 & 6 & 0 & 2.833968 & -2.574191 & -1.779579 \\
11 & 1 & 0 & 1.766249 & -2.795053 & -1.903559 \\
12 & 1 & 0 & 3.109325 & -1.761414 & -2.460173 \\
13 & 1 & 0 & 3.375499 & -3.465100 & -2.110389 \\
14 & 6 & 0 & 2.533653 & -0.963227 & 0.183816
\end{tabular}




\begin{tabular}{|c|c|c|c|c|c|}
\hline 15 & 1 & 0 & 2. 695004 & -0.898851 & 1. 260742 \\
\hline 16 & 6 & 0 & 3. 026277 & 0.335754 & -0.477878 \\
\hline 17 & 1 & 0 & 2. 898271 & 0.254902 & -1.565248 \\
\hline 18 & 1 & 0 & 4. 105097 & 0.422514 & -0.285228 \\
\hline 19 & 7 & 0 & 1. 078354 & -1.016110 & -0.050422 \\
\hline 20 & 1 & 0 & 0.493070 & 1. 511169 & 0.291384 \\
\hline 21 & 7 & 0 & 2. 300811 & 1.526863 & -0.022907 \\
\hline 22 & 6 & 0 & 2. 441104 & 2. 636176 & -0.992239 \\
\hline 23 & 1 & 0 & 2. 077628 & 2. 260395 & -1.955701 \\
\hline 24 & 1 & 0 & 1. 745809 & 3. 424341 & -0.688346 \\
\hline 25 & 6 & 0 & 2. 715788 & 1. 898540 & 1. 347686 \\
\hline 26 & 1 & 0 & 2. 429013 & 1. 071948 & 2. 009347 \\
\hline 27 & 1 & 0 & 3. 812957 & 1. 982318 & 1. 404882 \\
\hline 28 & 6 & 0 & 2. 064739 & 3. 175496 & 1. 864967 \\
\hline 29 & 1 & 0 & 2. 431017 & 4. 063652 & 1. 343774 \\
\hline 30 & 1 & 0 & 0.975539 & 3. 137063 & 1. 770444 \\
\hline 31 & 1 & 0 & 2. 309326 & 3. 295522 & 2. 923211 \\
\hline 32 & 6 & 0 & 3.848440 & 3. 204649 & -1.154165 \\
\hline 33 & 1 & 0 & 4. 559556 & 2. 446829 & -1.497830 \\
\hline 34 & 1 & 0 & 3. 836304 & 4. 004878 & -1.898559 \\
\hline 35 & 1 & 0 & 4. 225893 & 3. 629572 & -0.219493 \\
\hline 36 & 1 & 0 & 0.841115 & -0.964225 & -1.040262 \\
\hline 37 & 6 & 0 & 0.075704 & -0.960709 & 0.763877 \\
\hline 38 & 6 & 0 & -1.299697 & -0.731718 & 0.148083 \\
\hline 39 & 6 & 0 & 0.261057 & -1.051035 & 2. 237124 \\
\hline 40 & 1 & 0 & 1. 266888 & -1.344229 & 2.531981 \\
\hline 41 & 1 & 0 & 0.015437 & -0.068556 & 2. 656691 \\
\hline 42 & 1 & 0 & -0.450401 & -1.771864 & 2. 648031 \\
\hline 43 & 6 & 0 & -1.514642 & -1.662306 & -1.059327 \\
\hline 44 & 1 & 0 & -0.806971 & -1.505734 & -1.879713 \\
\hline 45 & 1 & 0 & -1.446958 & -2.709446 & -0.745805 \\
\hline 46 & 1 & 0 & -2.512742 & -1.502646 & -1.472956 \\
\hline 47 & 6 & 0 & -1.284232 & 0.831543 & -0.215330 \\
\hline 48 & 8 & 0 & -0.421282 & 1. 473000 & 0.679164 \\
\hline 49 & 6 & 0 & -5.963534 & 0.465790 & -0.770596 \\
\hline 50 & 6 & 0 & -6.015600 & -0.790209 & -0.169414 \\
\hline 51 & 6 & 0 & -4.891520 & -1.303064 & 0.475986 \\
\hline 52 & 6 & 0 & -3.722513 & -0.552627 & 0.532974 \\
\hline 53 & 6 & 0 & -3.685232 & 0.693206 & -0.087204 \\
\hline 54 & 6 & 0 & -4.790376 & 1. 217708 & -0.742158 \\
\hline 55 & 1 & 0 & -6.840479 & 0.863528 & -1.270739 \\
\hline 56 & 1 & 0 & -6.931408 & -1.370420 & -0.201272 \\
\hline 57 & 1 & 0 & -4.926962 & -2.282105 & 0.946916 \\
\hline 58 & 1 & 0 & -4.723116 & 2. 197911 & -1.201849 \\
\hline
\end{tabular}




\begin{tabular}{rrrrrr}
59 & 8 & 0 & -2.535341 & 1.440027 & 0.019671 \\
60 & 6 & 0 & -2.431792 & -0.970144 & 1.172728 \\
61 & 1 & 0 & -2.252518 & -0.352678 & 2.061224 \\
62 & 1 & 0 & -2.464532 & -2.021090 & 1.480751 \\
63 & 6 & 0 & -0.897751 & 1.134851 & -1.660105 \\
64 & 1 & 0 & -0.887872 & 2.222637 & -1.764308 \\
65 & 1 & 0 & 0.099741 & 0.761066 & -1.923461 \\
66 & 1 & 0 & -1.629916 & 0.722518 & -2.358420 \\
\hline
\end{tabular}

(11) $\mathrm{TS}_{3}$

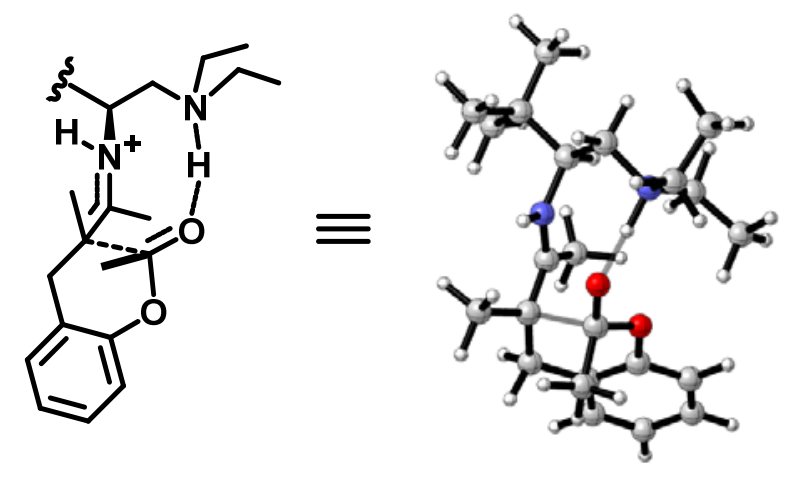

$\mathrm{E}_{\mathrm{sol}}=-1159.565358$

Zero-point correction=

Thermal correction to Energy=

0.600008 (Hartree/Particle)

Thermal correction to Enthalpy=

0.628193

Thermal correction to Gibbs Free Energy=

Sum of electronic and zero-point Energies=

0.629137

Sum of electronic and thermal Energies=

0.544983

$-1158.554813$

$-1158.526628$

$-1158.525683$

Sum of electronic and thermal Enthalpies=

$-1158.609838$

Sum of electronic and thermal Free Energies=

\section{E (Therma1) \\ KCal/Mol}

Total

394. 197

Electronic

Translational

Rotational

Vibrational
0.000

0.889

0.889

392. 420
CV

Cal/Mol-Kelvin

113. 248

0.000

2. 981

2. 981

107. 287
$\mathrm{S}$

Cal/Mol-Kelvin

177. 118

0.000

43. 660

35. 387

98. 071

Standard orientation:

\begin{tabular}{|c|c|c|c|c|c|}
\hline \multirow{2}{*}{$\begin{array}{l}\text { Center } \\
\text { Number }\end{array}$} & \multirow{2}{*}{$\begin{array}{l}\text { Atomic } \\
\text { Number }\end{array}$} & \multirow{2}{*}{$\begin{array}{l}\text { Atomic } \\
\text { Type }\end{array}$} & \multicolumn{3}{|c|}{ Coordinates (Angstroms) } \\
\hline & & & $X$ & $\mathrm{Y}$ & Z \\
\hline 1 & 6 & 0 & -3.325567 & -1.789365 & -0.687928 \\
\hline 2 & 6 & 0 & -4.498007 & -1.273546 & -1.532998 \\
\hline 3 & 1 & 0 & -4.152218 & -0.847255 & -2.482651 \\
\hline
\end{tabular}




\begin{tabular}{|c|c|c|c|c|c|}
\hline 4 & 1 & 0 & -5.171876 & -2.101651 & -1.770865 \\
\hline 5 & 1 & 0 & -5.090076 & -0.517848 & -1.006627 \\
\hline 6 & 6 & 0 & -2.706457 & -3.004831 & -1.388113 \\
\hline 7 & 1 & 0 & -2.403081 & -2.760957 & -2.41317 \\
\hline 8 & 1 & 0 & -1.834652 & -3.382325 & -0.84279 \\
\hline 9 & 1 & 0 & -3.438090 & -3.816001 & -1.4463 \\
\hline 10 & 6 & 0 & -3.831801 & -2.215895 & 0.6957 \\
\hline 11 & 1 & 0 & -3.032744 & -2.654418 & 1. 303863 \\
\hline 12 & 1 & 0 & -4.287318 & -1.390505 & 1. 254075 \\
\hline 13 & 1 & 0 & -4.602879 & -2.983159 & 0.5791 \\
\hline 14 & 6 & 0 & -2.229286 & -0.688245 & -0.5809 \\
\hline 15 & 1 & 0 & -1.816214 & -0.540612 & -1.582418 \\
\hline 16 & 6 & 0 & -2.782765 & 0.653599 & -0.07681 \\
\hline 17 & 1 & 0 & -3.263709 & 0.515549 & 0.8971 \\
\hline 18 & 1 & 0 & -3.532542 & 1. 031691 & -0.775369 \\
\hline 19 & 7 & 0 & -1.140944 & -1.102713 & $0.3010 s$ \\
\hline 20 & 7 & 0 & -1.743178 & 1. 705820 & 0.106505 \\
\hline 21 & 6 & 0 & -2.255695 & 2.827865 & 0.96144 \\
\hline 22 & 1 & 0 & -2.576665 & 2. 362490 & 1. 8972 \\
\hline 23 & 1 & 0 & -1.392627 & 3. 450995 & 1. 200713 \\
\hline 24 & 6 & 0 & -1.150634 & 2. 152647 & -1.193125 \\
\hline 25 & 1 & 0 & -0.622766 & 1. 284921 & -1.59553 \\
\hline 26 & 1 & 0 & -1.971872 & 2. 399965 & -1.874353 \\
\hline 27 & 6 & 0 & -0.176125 & 3. 308988 & -1.037417 \\
\hline 28 & 1 & 0 & -0.680582 & 4. 248929 & -0.801617 \\
\hline 29 & 1 & 0 & 0.565615 & 3. 086350 & -0.265975 \\
\hline 30 & 1 & 0 & 0.351555 & 3. 446145 & -1.984527 \\
\hline 31 & 6 & 0 & -3.375204 & 3. 635098 & 0.326550 \\
\hline 32 & 1 & 0 & -4.267165 & 3. 031832 & 0.136604 \\
\hline 33 & 1 & 0 & -3.663398 & 4. 435432 & 1. 012204 \\
\hline 34 & 1 & 0 & -3.063785 & 4. 103448 & -0.611560 \\
\hline 35 & 1 & 0 & -1.354421 & -1.065550 & 1. 293228 \\
\hline 36 & 6 & 0 & 0.138431 & -1.357403 & 0.006722 \\
\hline 37 & 6 & 0 & 1. 050718 & -1.488953 & 1. 075721 \\
\hline 38 & 6 & 0 & 0.552080 & -1.373835 & -1.436332 \\
\hline 39 & 1 & 0 & 0.795568 & -0.361777 & -1.782693 \\
\hline 40 & 1 & 0 & 1. 449260 & -1.977678 & -1.571572 \\
\hline 41 & 1 & 0 & -0.235029 & -1.788386 & -2.068490 \\
\hline 42 & 6 & 0 & 0.487719 & -2.034549 & 2. 382757 \\
\hline 43 & 1 & 0 & -0.127823 & -1.292892 & 2. 907770 \\
\hline 44 & 1 & 0 & -0.119486 & -2.932816 & 2. 215496 \\
\hline 45 & 1 & 0 & 1. 297953 & -2.312604 & 3. 060171 \\
\hline 46 & 6 & 0 & 1. 169166 & 0.477217 & 1. 4920 \\
\hline 47 & 8 & 0 & 0.000784 & 0.876983 & 1. 7780 \\
\hline
\end{tabular}




\begin{tabular}{rrrrrr}
48 & 6 & 0 & 4.719272 & 0.963339 & -1.663846 \\
49 & 6 & 0 & 5.158233 & -0.351280 & -1.511203 \\
50 & 6 & 0 & 4.420327 & -1.233960 & -0.734833 \\
51 & 6 & 0 & 3.241248 & -0.846620 & -0.087468 \\
52 & 6 & 0 & 2.830216 & 0.481167 & -0.248729 \\
53 & 6 & 0 & 3.556213 & 1.377291 & -1.031908 \\
54 & 1 & 0 & 5.284592 & 1.664824 & -2.268358 \\
55 & 1 & 0 & 6.069185 & -0.685539 & -1.995939 \\
56 & 1 & 0 & 4.757286 & -2.261759 & -0.620765 \\
57 & 1 & 0 & 3.191130 & 2.395466 & -1.122778 \\
58 & 8 & 0 & 1.650436 & 0.981244 & 0.277722 \\
59 & 6 & 0 & 2.483954 & -1.868833 & 0.738877 \\
60 & 1 & 0 & 2.526484 & -2.839219 & 0.224530 \\
61 & 1 & 0 & 3.020677 & -2.025672 & 1.681830 \\
62 & 6 & 0 & 2.191454 & 0.497561 & 2.615147 \\
63 & 1 & 0 & 3.155875 & 0.070425 & 2.343498 \\
64 & 1 & 0 & 2.340499 & 1.554142 & 2.860201 \\
65 & 1 & 0 & 1.787699 & -0.000697 & 3.496226 \\
66 & 1 & 0 & -0.918207 & 1.284417 & 0.720695 \\
\hline
\end{tabular}

(12) TS3a

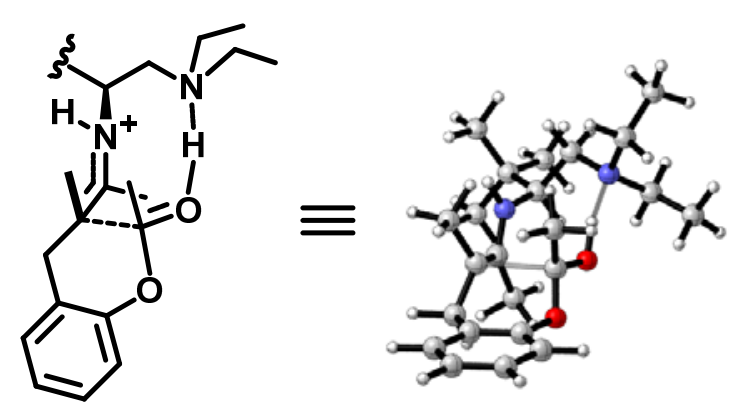

$\mathrm{E}_{\mathrm{sol}}=-1159.546747$

Zero-point correction=

0. 598202 (Hartree/Particle)

Thermal correction to Energy=

0.626816

Thermal correction to Enthalpy=

0.627760

Thermal correction to Gibbs Free Energy=

Sum of electronic and zero-point Energies=

0.541331

Sum of electronic and thermal Energies=

$-1158.539586$

$-1158.510972$

$-1158.510028$

Sum of electronic and thermal Enthalpies=

$-1158.596457$

Sum of electronic and thermal Free Energies=

$\begin{array}{lccc} & \text { E (Therma1) } & \text { CV } & \text { S } \\ & \text { KCa1/Mo1 } & \text { Cal/Mo1-Ke1vin } & \text { Cal/Mo1-Ke1vin } \\ \text { Total } & 393.333 & 113.626 & 181.905 \\ \text { Electronic } & 0.000 & 0.000 & 0.000 \\ \text { Translational } & 0.889 & 2.981 & 43.660\end{array}$


Rotational

0.889

2. 981

35. 613

Vibrational

391.555

107. 664

102. 632

Standard orientation:

\begin{tabular}{|c|c|c|c|c|c|}
\hline \multirow{2}{*}{$\begin{array}{l}\text { Center } \\
\text { Number }\end{array}$} & \multirow{2}{*}{$\begin{array}{l}\text { Atomic } \\
\text { Number }\end{array}$} & \multirow{2}{*}{$\begin{array}{l}\text { Atomic } \\
\text { Type }\end{array}$} & \multicolumn{3}{|c|}{ Coordinates (Angstroms) } \\
\hline & & & $X$ & Y & Z \\
\hline 1 & 6 & 0 & -3.469973 & -1.997378 & -0.018912 \\
\hline 2 & 6 & 0 & -4.802654 & -1.596086 & -0.666722 \\
\hline 3 & 1 & 0 & -4.654516 & -1.225635 & -1.688219 \\
\hline 4 & 1 & 0 & -5.459393 & -2.469105 & -0.724735 \\
\hline 5 & 1 & 0 & -5.337393 & -0.830533 & -0.097120 \\
\hline 6 & 6 & 0 & -2.918511 & -3.218387 & -0.764695 \\
\hline 7 & 1 & 0 & -2.774906 & -3.002000 & -1.830094 \\
\hline 8 & 1 & 0 & -1.963880 & -3.553208 & -0.346249 \\
\hline 9 & 1 & 0 & -3.624540 & -4.050701 & -0.690063 \\
\hline 10 & 6 & 0 & -3.694913 & -2.356939 & 1. 454252 \\
\hline 11 & 1 & 0 & -2.764925 & -2.669396 & 1. 944414 \\
\hline 12 & 1 & 0 & -4.128691 & -1.525360 & 2. 020611 \\
\hline 13 & 1 & 0 & -4.392077 & -3.196999 & 1. 528839 \\
\hline 14 & 6 & 0 & -2.454916 & -0.830864 & -0.162035 \\
\hline 15 & 1 & 0 & -2.364038 & -0.606947 & -1.227249 \\
\hline 16 & 6 & 0 & -2.867484 & 0.455342 & 0.570300 \\
\hline 17 & 1 & 0 & -2.706611 & 0.318522 & 1. 646909 \\
\hline 18 & 1 & 0 & -3.939336 & 0.639022 & 0.431020 \\
\hline 19 & 7 & 0 & -1.122322 & -1.194318 & 0.331406 \\
\hline 20 & 1 & 0 & -0.677245 & 1. 301589 & -0.357211 \\
\hline 21 & 7 & 0 & -2.089449 & 1. 634306 & 0.134786 \\
\hline 22 & 6 & 0 & -1.988542 & 2. 647781 & 1. 213181 \\
\hline 23 & 1 & 0 & -1.559722 & 2. 137693 & 2. 083253 \\
\hline 24 & 1 & 0 & -1.255802 & 3. 395457 & 0.893676 \\
\hline 25 & 6 & 0 & -2.671779 & 2. 188892 & -1.116489 \\
\hline 26 & 1 & 0 & -2.613056 & 1. 398173 & -1.871962 \\
\hline 27 & 1 & 0 & -3.737756 & 2. 404759 & -0.959796 \\
\hline 28 & 6 & 0 & -1.947844 & 3. 424791 & -1.631694 \\
\hline 29 & 1 & 0 & -2.095419 & 4. 289843 & -0.979649 \\
\hline 30 & 1 & 0 & -0.875524 & 3. 240204 & -1.740339 \\
\hline 31 & 1 & 0 & -2.345703 & 3. 685177 & -2.615339 \\
\hline 32 & 6 & 0 & -3.299355 & 3. 319875 & 1. 607059 \\
\hline 33 & 1 & 0 & -4.050071 & 2. 589691 & 1. 924471 \\
\hline 34 & 1 & 0 & -3.123146 & 3. 998454 & 2. 445307 \\
\hline 35 & 1 & 0 & -3.718105 & 3. 910088 & 0.787369 \\
\hline 36 & 1 & 0 & -1.047273 & -1.192414 & 1. 342671 \\
\hline 37 & 6 & 0 & 0.025449 & -1.393858 & -0.329990 \\
\hline
\end{tabular}




\begin{tabular}{|c|c|c|c|c|c|}
\hline 38 & 6 & 0 & 1. 244142 & -1.291676 & 0.357653 \\
\hline 39 & 6 & 0 & 0.001061 & -1.594953 & -1.815513 \\
\hline 40 & 1 & 0 & -0.956270 & -1.347427 & -2.268253 \\
\hline 41 & 1 & 0 & 0.771753 & -0.984600 & -2.290977 \\
\hline 42 & 1 & 0 & 0.211210 & -2.648765 & -2.031274 \\
\hline 43 & 6 & 0 & 1. 262076 & -1.378940 & 1. 871588 \\
\hline 44 & 1 & 0 & 0.579215 & -0.690905 & 2. 380442 \\
\hline 45 & 1 & 0 & 1. 014541 & -2.397128 & 2. 199861 \\
\hline 46 & 1 & 0 & 2. 266172 & -1.158035 & 2. 246265 \\
\hline 47 & 6 & 0 & 1. 276619 & 0.820941 & -0.275441 \\
\hline 48 & 8 & 0 & 0.198625 & 1. 129417 & -0.942111 \\
\hline 49 & 6 & 0 & 5. 795923 & 0.919976 & 0.089849 \\
\hline 50 & 6 & 0 & 5. 957201 & -0.380761 & 0.559603 \\
\hline 51 & 6 & 0 & 4. 909593 & -1.296962 & 0.470408 \\
\hline 52 & 6 & 0 & 3. 689652 & -0.924809 & -0.088634 \\
\hline 53 & 6 & 0 & 3. 558456 & 0.387148 & -0.531699 \\
\hline 54 & 6 & 0 & 4. 578653 & 1. 318765 & -0.461048 \\
\hline 55 & 1 & 0 & 6.614583 & 1. 628806 & 0.152813 \\
\hline 56 & 1 & 0 & 6. 903913 & -0.687169 & 0.991817 \\
\hline 57 & 1 & 0 & 5. 042757 & -2.315006 & 0.826831 \\
\hline 58 & 1 & 0 & 4. 419280 & 2. 323507 & -0.838396 \\
\hline 59 & 8 & 0 & 2. 332749 & 0.750954 & -1.105008 \\
\hline 60 & 6 & 0 & 2. 514355 & -1.854850 & -0.266416 \\
\hline 61 & 1 & 0 & 2. 382941 & -2.047274 & -1.334644 \\
\hline 62 & 1 & 0 & 2. 745571 & -2.822136 & 0.198033 \\
\hline 63 & 6 & 0 & 1. 461996 & 1. 473890 & 1. 069117 \\
\hline 64 & 1 & 0 & 1. 619513 & 2. 541886 & 0.875203 \\
\hline 65 & 1 & 0 & 0.553239 & 1. 364200 & 1. 663240 \\
\hline 66 & 1 & 0 & 2. 316878 & 1. 085151 & 1. 619798 \\
\hline
\end{tabular}

(13) TS3b

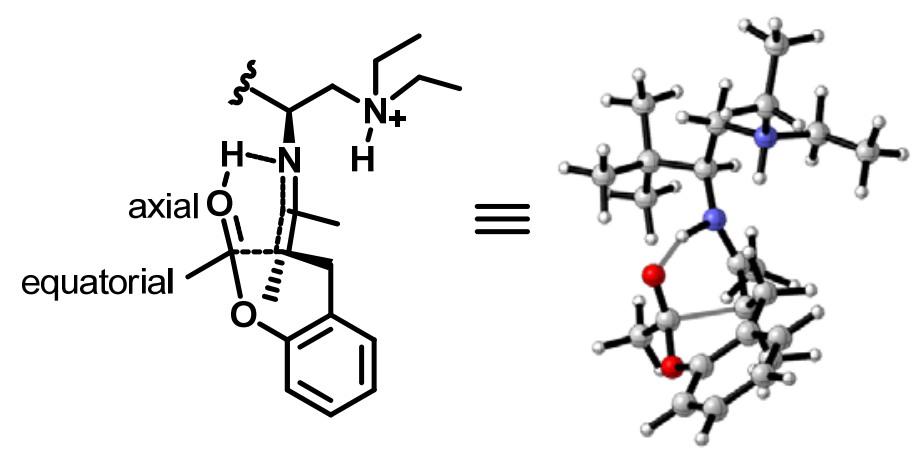

$E_{\text {sol }}=-1159.541174$

Zero-point correction=

0. 596876 (Hartree/Particle)

Thermal correction to Energy=

0. 625665

Thermal correction to Enthalpy=

0. 626609 
Thermal correction to Gibbs Free Energy=

Sum of electronic and zero-point Energies=

Sum of electronic and thermal Energies=

Sum of electronic and thermal Enthalpies=

Sum of electronic and thermal Free Energies=
0.540013

$-1158.517019$

$-1158.488230$

$-1158.487286$

$-1158.573881$
E (Therma1)

$\mathrm{KCa} 1 / \mathrm{Mol}$

Total 392. 611

Electronic

Translational

0.000

0. 889

0.889

Rotational

Vibrational
CV Cal/Mo1-Kelvin 113. 932

0.000

2. 981

2. 981 107. 970
$\mathrm{S}$

Cal/Mol-Kelvin

182. 256

0.000

43. 660

35. 592

103. 004

Standard orientation:

\begin{tabular}{ccccc} 
Center & Atomic & Atomic & \multicolumn{2}{c}{ Coordinates (Angstroms) } \\
Number & Number & Type & $\mathrm{X}$ & $\mathrm{Y}$
\end{tabular}

$\begin{array}{rrrrrr}1 & 6 & 0 & 2.938108 & -1.840463 & -0.710345 \\ 2 & 6 & 0 & 4.430448 & -1.653178 & -1.025659 \\ 3 & 1 & 0 & 5.006912 & -1.399771 & -0.127260 \\ 4 & 1 & 0 & 4.838291 & -2.586495 & -1.424113 \\ 5 & 1 & 0 & 4.605169 & -0.881158 & -1.782912 \\ 6 & 6 & 0 & 2.792989 & -3.080366 & 0.177008 \\ 7 & 1 & 0 & 3.406380 & -2.999229 & 1.082335 \\ 8 & 1 & 0 & 1.755524 & -3.245026 & 0.479427 \\ 9 & 1 & 0 & 3.121994 & -3.969331 & -0.369243 \\ 10 & 6 & 0 & 2.157845 & -2.053529 & -2.012738 \\ 11 & 1 & 0 & 1.096218 & -2.247734 & -1.835488 \\ 12 & 1 & 0 & 2.229738 & -1.196497 & -2.692785 \\ 13 & 1 & 0 & 2.576319 & -2.913720 & -2.544384 \\ 14 & 6 & 0 & 2.421184 & -0.598919 & 0.076833 \\ 15 & 1 & 0 & 2.897928 & -0.628755 & 1.064763 \\ 16 & 6 & 0 & 2.862224 & 0.708673 & -0.622677 \\ 17 & 1 & 0 & 2.645848 & 0.657684 & -1.693527 \\ 18 & 1 & 0 & 3.925595 & 0.907514 & -0.482764 \\ 19 & 7 & 0 & 0.975973 & -0.502912 & 0.240010 \\ 20 & 1 & 0 & 1.132437 & 1.503565 & 0.004492 \\ 21 & 7 & 0 & 2.092596 & 1.882077 & -0.090322 \\ 22 & 6 & 0 & 2.044619 & 3.040069 & -1.052358 \\ 23 & 1 & 0 & 1.661439 & 2.630712 & -1.990148 \\ 24 & 1 & 0 & 1.300654 & 3.739177 & -0.666187 \\ 25 & 6 & 0 & 2.524945 & 2.249373 & 1.302310 \\ 26 & 1 & 0 & 2.415870 & 1.338265 & 1.895795\end{array}$




\begin{tabular}{|c|c|c|c|c|c|}
\hline 27 & 1 & 0 & 3.587186 & 2. 500291 & 1. 254633 \\
\hline 28 & 6 & 0 & 1. 692729 & 3. 372495 & 1. 895301 \\
\hline 29 & 1 & 0 & 1.882403 & 4. 334099 & 1. 412761 \\
\hline 30 & 1 & 0 & 0.622288 & 3. 148708 & 1. 834471 \\
\hline 31 & 1 & 0 & 1. 949281 & 3. 478010 & 2. 951750 \\
\hline 32 & 6 & 0 & 3. 393948 & 3. 707727 & -1.245740 \\
\hline 33 & 1 & 0 & 4. 145095 & 3. 011654 & -1.627988 \\
\hline 34 & 1 & 0 & 3. 281664 & 4. 506154 & -1.982585 \\
\hline 35 & 1 & 0 & 3. 765738 & 4. 162145 & -0.323495 \\
\hline 36 & 1 & 0 & 0.239069 & -0.668645 & -0.671804 \\
\hline 37 & 6 & 0 & 0.251982 & -0.859839 & 1. 317056 \\
\hline 38 & 6 & 0 & -1.139104 & -0.584250 & 1. 254016 \\
\hline 39 & 6 & 0 & 0.826796 & -1.629208 & 2. 472808 \\
\hline 40 & 1 & 0 & 1.915817 & -1.671086 & 2. 465987 \\
\hline 41 & 1 & 0 & 0.485732 & -1.200877 & 3. 419303 \\
\hline 42 & 1 & 0 & 0.459917 & -2.662776 & 2. 442219 \\
\hline 43 & 6 & 0 & -2.017113 & -1.028177 & 2. 403518 \\
\hline 44 & 1 & 0 & -1.792384 & -2.045228 & 2. 735671 \\
\hline 45 & 1 & 0 & -1.914100 & -0.362109 & 3. 270374 \\
\hline 46 & 1 & 0 & -3.066999 & -1.007005 & 2. 098518 \\
\hline 47 & 6 & 0 & -1.573338 & -1.656616 & -0.366366 \\
\hline 48 & 8 & 0 & -0.890159 & -1.062850 & -1.281489 \\
\hline 49 & 6 & 0 & -5.406463 & 1. 016358 & -1.203861 \\
\hline 50 & 6 & 0 & -4.821546 & 2. 162476 & -0.664748 \\
\hline 51 & 6 & 0 & -3.582778 & 2. 074379 & -0.043676 \\
\hline 52 & 6 & 0 & -2.897926 & 0.859821 & 0.044020 \\
\hline 53 & 6 & 0 & -3.502671 & -0.266274 & -0.506946 \\
\hline 54 & 6 & 0 & -4.749623 & -0.203236 & -1.121146 \\
\hline 55 & 1 & 0 & -6.377332 & 1. 073307 & -1.684895 \\
\hline 56 & 1 & 0 & -5.334476 & 3. 116632 & -0.721811 \\
\hline 57 & 1 & 0 & -3.131193 & 2. 963751 & 0.391059 \\
\hline 58 & 1 & 0 & -5.178917 & -1.115427 & -1.521095 \\
\hline 59 & 8 & 0 & -2.942586 & -1.515342 & -0.370736 \\
\hline 60 & 6 & 0 & -1.537733 & 0.783592 & 0.686367 \\
\hline 61 & 1 & 0 & -0.806941 & 1. 097925 & -0.067963 \\
\hline 62 & 1 & 0 & -1.502901 & 1. 512743 & 1. 510509 \\
\hline 63 & 6 & 0 & -1.280617 & -3.105488 & -0.045326 \\
\hline 64 & 1 & 0 & -1.630884 & -3.706527 & -0.890337 \\
\hline 65 & 1 & 0 & -0.205004 & -3.258751 & 0.055805 \\
\hline 66 & 1 & 0 & -1.800833 & -3.437328 & 0.855002 \\
\hline
\end{tabular}

\section{(14) Int 5}



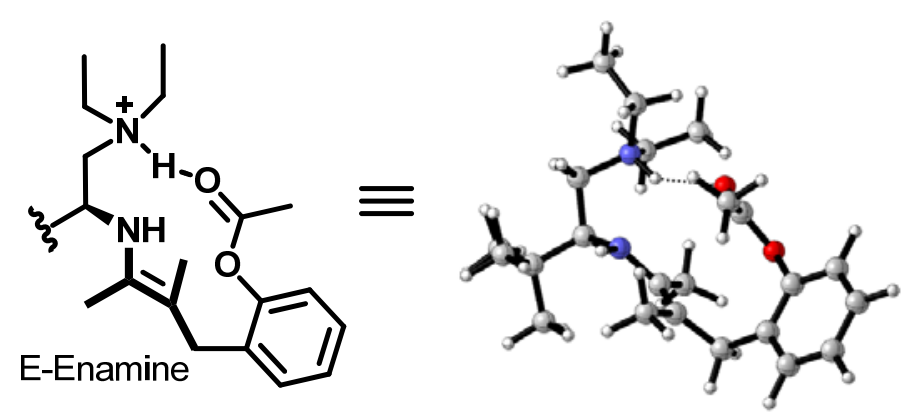

$\mathrm{E}_{\mathrm{sol}}=-1159.586895$

Zero-point correction=

0.602015 (Hartree/Particle)

Thermal correction to Energy=

0.631921

Thermal correction to Enthalpy=

0.632865

Thermal correction to Gibbs Free Energy=

Sum of electronic and zero-point Energies=

0.543134

Sum of electronic and thermal Energies=

$-1158.575145$

$-1158.545240$

Sum of electronic and thermal Enthalpies=

Sum of electronic and thermal Free Energies=

$-1158.544295$

$-1158.634026$

\begin{tabular}{lccr} 
& E (Thermal) & CV & \multicolumn{1}{c}{ S } \\
& KCa1/Mol & Cal/Mo1-Kelvin & Ca1/Mo1-Kelvin \\
Total & 396.536 & 115.761 & 188.854 \\
Electronic & 0.000 & 0.000 & 0.000 \\
Translational & 0.889 & 2.981 & 43.660 \\
Rotational & 0.889 & 2.981 & 35.669 \\
Vibrational & 394.759 & 109.799 & 109.526 \\
Standard orientation: & & &
\end{tabular}

\begin{tabular}{|c|c|c|c|c|c|}
\hline \multirow{2}{*}{$\begin{array}{l}\text { Center } \\
\text { Number }\end{array}$} & \multirow{2}{*}{$\begin{array}{l}\text { Atomic } \\
\text { Number }\end{array}$} & \multirow{2}{*}{$\begin{array}{c}\text { Atomic } \\
\text { Type }\end{array}$} & \multicolumn{3}{|c|}{ Coordinates (Angstroms) } \\
\hline & & & $X$ & Y & Z \\
\hline 1 & 6 & 0 & 3. 060523 & -2.336514 & -0.094376 \\
\hline 2 & 6 & 0 & 2. 403498 & -0.957561 & 0.182757 \\
\hline 3 & 1 & 0 & 2.417831 & -0.825557 & 1. 269972 \\
\hline 4 & 6 & 0 & 3. 158872 & 0.223884 & -0.449565 \\
\hline 5 & 1 & 0 & 3. 135566 & 0.143783 & -1.540171 \\
\hline 6 & 1 & 0 & 4. 198597 & 0.290641 & -0.123720 \\
\hline 7 & 7 & 0 & 1. 018872 & -0.843559 & -0.263598 \\
\hline 8 & 1 & 0 & 1. 458272 & 1. 267895 & 0.005427 \\
\hline 9 & 7 & 0 & 2. 468828 & 1. 505794 & -0.079876 \\
\hline 10 & 6 & 0 & 2.509100 & 2.543006 & -1.168167 \\
\hline 11 & 1 & 0 & 2.107867 & 2. 046006 & -2.055515 \\
\hline 12 & 1 & 0 & 1. 797850 & 3. 318119 & -0.877221 \\
\hline 13 & 6 & 0 & 2.948147 & 2. 015923 & 1. 252557 \\
\hline 14 & 1 & 0 & 2. 827457 & 1. 186906 & 1. 954127 \\
\hline
\end{tabular}




\begin{tabular}{|c|c|c|c|c|c|}
\hline 15 & 1 & 0 & 4. 018592 & 2. 209411 & 1. 146938 \\
\hline 16 & 6 & 0 & 2. 192140 & 3. 242564 & 1. 732658 \\
\hline 17 & 1 & 0 & 2. 423335 & 4. 126008 & 1. 132005 \\
\hline 18 & 1 & 0 & 1. 112355 & 3. 074165 & 1. 718242 \\
\hline 19 & 1 & 0 & 2. 498205 & 3. 455310 & 2. 759622 \\
\hline 20 & 6 & 0 & 3. 899217 & 3. 097057 & -1.418961 \\
\hline 21 & 1 & 0 & 4. 621258 & 2. 307830 & -1.648922 \\
\hline 22 & 1 & 0 & 3.858575 & 3. 767234 & -2.280587 \\
\hline 23 & 1 & 0 & 4. 269760 & 3. 678209 & -0.570400 \\
\hline 24 & 1 & 0 & 0.886749 & -1.280480 & -1.169290 \\
\hline 25 & 6 & 0 & -0.090398 & -1.039893 & 0.597738 \\
\hline 26 & 6 & 0 & -1.232342 & -1.566432 & 0.114904 \\
\hline 27 & 6 & 0 & 0.100268 & -0.511148 & 1. 993970 \\
\hline 28 & 1 & 0 & 0.612156 & 0.457715 & 1. 972178 \\
\hline 29 & 1 & 0 & -0.849714 & -0.338820 & 2. 494511 \\
\hline 30 & 1 & 0 & 0.688891 & -1.200005 & 2. 611298 \\
\hline 31 & 6 & 0 & -1.313118 & -2.117580 & -1.293602 \\
\hline 32 & 1 & 0 & -0.731844 & -3.044141 & -1.400044 \\
\hline 33 & 1 & 0 & -2.348271 & -2.356824 & -1.554016 \\
\hline 34 & 1 & 0 & -0.955138 & -1.417441 & -2.059855 \\
\hline 35 & 6 & 0 & -2.510212 & -1.661013 & 0.920050 \\
\hline 36 & 1 & 0 & -2.336862 & -1.441502 & 1. 975511 \\
\hline 37 & 1 & 0 & -2.871760 & -2.696257 & 0.885799 \\
\hline 38 & 6 & 0 & -3.628866 & -0.770421 & 0.410017 \\
\hline 39 & 6 & 0 & -4.854611 & -1.308940 & 0.011890 \\
\hline 40 & 6 & 0 & -3.504680 & 0.619931 & 0.345361 \\
\hline 41 & 6 & 0 & -5.905836 & -0.500588 & -0.413581 \\
\hline 42 & 1 & 0 & -4.986417 & -2.387214 & 0.050697 \\
\hline 43 & 6 & 0 & -4.541796 & 1. 448204 & -0.057057 \\
\hline 44 & 6 & 0 & -5.753924 & 0.882119 & -0.441145 \\
\hline 45 & 1 & 0 & -6.846158 & -0.951649 & -0.712714 \\
\hline 46 & 1 & 0 & -4.392856 & 2. 523832 & -0.053978 \\
\hline 47 & 1 & 0 & -6.572980 & 1. 520114 & -0.755312 \\
\hline 48 & 8 & 0 & -2.296738 & 1. 211650 & 0.742327 \\
\hline 49 & 6 & 0 & -1.380733 & 1. 532600 & -0.185123 \\
\hline 50 & 6 & 0 & -1.725523 & 1. 356432 & -1.640670 \\
\hline 51 & 1 & 0 & -2.273320 & 0.432599 & -1.829637 \\
\hline 52 & 1 & 0 & -2.361609 & 2. 187557 & -1.963037 \\
\hline 53 & 1 & 0 & -0.798415 & 1. 379771 & -2.213686 \\
\hline 54 & 8 & 0 & -0.322114 & 1. 999787 & 0.186700 \\
\hline 55 & 6 & 0 & 3. 054960 & -2.686432 & -1.587693 \\
\hline 56 & 1 & 0 & 3. 524133 & -3.663807 & -1.735510 \\
\hline 57 & 1 & 0 & 2. 039378 & -2.763711 & -1.994716 \\
\hline 58 & 1 & 0 & 3. 618786 & -1.965665 & -2.19040 \\
\hline
\end{tabular}




$\begin{array}{rrrrrr}59 & 6 & 0 & 2.260991 & -3.394660 & 0.676592 \\ 60 & 1 & 0 & 2.275412 & -3.191320 & 1.753999 \\ 61 & 1 & 0 & 1.215713 & -3.429849 & 0.351874 \\ 62 & 1 & 0 & 2.698079 & -4.385354 & 0.519362 \\ 63 & 6 & 0 & 4.507032 & -2.331688 & 0.418888 \\ 64 & 1 & 0 & 4.915510 & -3.346239 & 0.390681 \\ 65 & 1 & 0 & 5.163991 & -1.704645 & -0.193236 \\ 66 & 1 & 0 & 4.561680 & -1.982865 & 1.457195\end{array}$

(15) Int5a
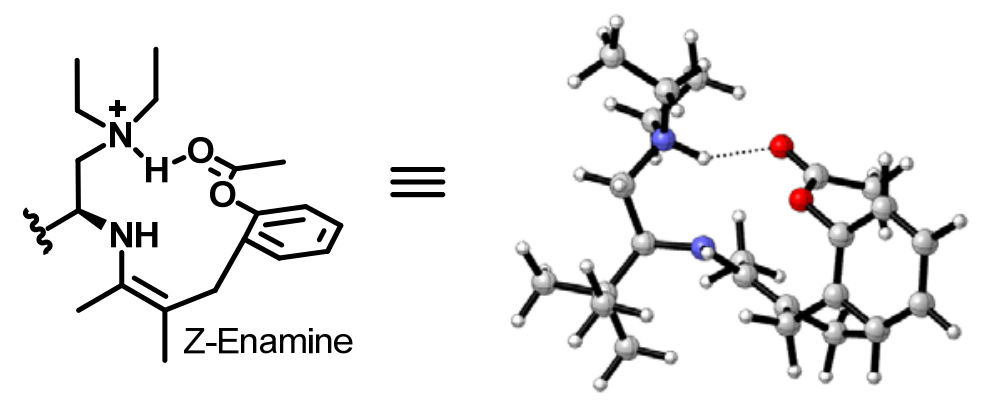

$\mathrm{E}_{\mathrm{sol}}=-1159.587832$

Zero-point correction=

Thermal correction to Energy=

0.601849 (Hartree/Particle)

Thermal correction to Enthalpy=

0.631703

Thermal correction to Gibbs Free Energy=

0. 632647

Sum of electronic and zero-point Energies=

0.543300

Sum of electronic and thermal Energies=

$-1158.579711$

$-1158.549857$

Sum of electronic and thermal Enthalpies=

$-1158.548913$

Sum of electronic and thermal Free Energies=

$-1158.638260$

$\begin{array}{lccc} & \text { E (Thermal) } & \text { CV } & \text { S } \\ & \text { KCal/Mol } & \text { Cal/Mol-Kelvin } & \text { Cal/Mo1-Kelvin } \\ \text { Total } & 396.399 & 115.901 & 188.046 \\ \text { Electronic } & 0.000 & 0.000 & 0.000 \\ \text { Translational } & 0.889 & 2.981 & 43.660 \\ \text { Rotational } & 0.889 & 2.981 & 35.547 \\ \text { Vibrational } & 394.622 & 109.939 & 108.839\end{array}$

Standard orientation:

\begin{tabular}{lllll} 
Center & Atomic & Atomic & \multicolumn{2}{c}{ Coordinates (Angstroms) } \\
Number & Number & Type & X & Y
\end{tabular}

$\begin{array}{rrrrrr}1 & 6 & 0 & -2.375153 & 2.507125 & -0.624828 \\ 2 & 6 & 0 & -2.115003 & 1.097975 & -0.019858 \\ 3 & 1 & 0 & -2.570256 & 1.100953 & 0.976935 \\ 4 & 6 & 0 & -2.767836 & -0.028359 & -0.842318\end{array}$




\begin{tabular}{|c|c|c|c|c|c|}
\hline 5 & 1 & 0 & -2.375229 & -0.023742 & -1.863895 \\
\hline 6 & 1 & 0 & -3.854739 & 0.061071 & -0.888029 \\
\hline 7 & 7 & 0 & -0.715480 & 0.725909 & 0.137939 \\
\hline 8 & 1 & 0 & -1.457333 & -1.250203 & 0.136602 \\
\hline 9 & 7 & 0 & -2.424104 & -1.359707 & -0.247333 \\
\hline 10 & 6 & 0 & -2.296442 & -2.451742 & -1.270561 \\
\hline 11 & 1 & 0 & -1.559266 & -2.088411 & -1.991522 \\
\hline 12 & 1 & 0 & -1.850788 & -3.304617 & -0.756084 \\
\hline 13 & 6 & 0 & -3.334832 & -1.701170 & 0.898114 \\
\hline 14 & 1 & 0 & -3.273010 & -0.858254 & 1. 590830 \\
\hline 15 & 1 & 0 & -4.352458 & -1.733748 & 0.499784 \\
\hline 16 & 6 & 0 & -2.955065 & -2.996204 & 1. 595588 \\
\hline 17 & 1 & 0 & -3.166710 & -3.872412 & 0.977443 \\
\hline 18 & 1 & 0 & -1.896961 & -3.001081 & 1. 869676 \\
\hline 19 & 1 & 0 & -3.551050 & -3.086321 & 2.506767 \\
\hline 20 & 6 & 0 & -3.613003 & -2.805683 & -1.936626 \\
\hline 21 & 1 & 0 & -4.077523 & -1.938945 & -2.416019 \\
\hline 22 & 1 & 0 & -3.426150 & -3.550784 & -2.713187 \\
\hline 23 & 1 & 0 & -4.325302 & -3.240896 & -1.230608 \\
\hline 24 & 1 & 0 & -0.196136 & 0.781475 & -0.730575 \\
\hline 25 & 6 & 0 & 0.061667 & 1. 028096 & 1. 280748 \\
\hline 26 & 6 & 0 & 1. 358424 & 1. 390834 & 1. 192059 \\
\hline 27 & 6 & 0 & -0.656967 & 0.784695 & 2. 580765 \\
\hline 28 & 1 & 0 & -1.122997 & -0.209755 & 2. 569854 \\
\hline 29 & 1 & 0 & 0.017898 & 0.830371 & 3. 433078 \\
\hline 30 & 1 & 0 & -1.441922 & 1. 528335 & 2. 758450 \\
\hline 31 & 6 & 0 & 2. 265040 & 1. 598425 & 2. 382319 \\
\hline 32 & 1 & 0 & 1. 984697 & 1. 016069 & 3. 261940 \\
\hline 33 & 1 & 0 & 3. 293297 & 1. 319432 & 2. 122004 \\
\hline 34 & 1 & 0 & 2. 292730 & 2. 654672 & 2. 677387 \\
\hline 35 & 6 & 0 & 2. 042092 & 1. 699358 & -0.126096 \\
\hline 36 & 1 & 0 & 2. 544045 & 2. 670018 & -0.022688 \\
\hline 37 & 1 & 0 & 1. 324681 & 1. 836282 & -0.943415 \\
\hline 38 & 6 & 0 & 3. 096210 & 0.694291 & -0.554030 \\
\hline 39 & 6 & 0 & 4. 413069 & 1. 094127 & -0.795509 \\
\hline 40 & 6 & 0 & 2. 805731 & -0.657141 & -0.749447 \\
\hline 41 & 6 & 0 & 5.383627 & 0.193935 & -1.226777 \\
\hline 42 & 1 & 0 & 4. 674882 & 2. 138439 & -0.646856 \\
\hline 43 & 6 & 0 & 3. 749933 & -1.570188 & -1.196569 \\
\hline 44 & 6 & 0 & 5. 051490 & -1.141291 & -1.435675 \\
\hline 45 & 1 & 0 & 6. 396325 & 0.538848 & -1.406725 \\
\hline 46 & 1 & 0 & 3.454874 & -2.602705 & -1.359009 \\
\hline 47 & 1 & 0 & 5. 797317 & -1.846836 & -1.785741 \\
\hline 48 & 8 & 0 & 1. 495728 & -1.108446 & -0.531991 \\
\hline
\end{tabular}




$\begin{array}{rrrrrr}49 & 6 & 0 & 1.191954 & -1.725996 & 0.629378 \\ 50 & 6 & 0 & 2.271606 & -1.925561 & 1.655771 \\ 51 & 1 & 0 & 2.960747 & -2.708908 & 1.325267 \\ 52 & 1 & 0 & 2.855558 & -1.015145 & 1.802600 \\ 53 & 1 & 0 & 1.796482 & -2.234148 & 2.585963 \\ 54 & 8 & 0 & 0.054885 & -2.122992 & 0.779584 \\ 55 & 6 & 0 & -1.709169 & 2.672958 & -1.995425 \\ 56 & 1 & 0 & -1.909929 & 3.677261 & -2.380662 \\ 57 & 1 & 0 & -0.619956 & 2.568136 & -1.936911 \\ 58 & 1 & 0 & -2.088942 & 1.964109 & -2.739616 \\ 59 & 6 & 0 & -1.798628 & 3.548967 & 0.341899 \\ 60 & 1 & 0 & -2.282513 & 3.486470 & 1.324033 \\ 61 & 1 & 0 & -0.720790 & 3.417249 & 0.481797 \\ 62 & 1 & 0 & -1.969273 & 4.557305 & -0.047809 \\ 63 & 6 & 0 & -3.886662 & 2.738098 & -0.760843 \\ 64 & 1 & 0 & -4.082236 & 3.785082 & -1.011061 \\ 65 & 1 & 0 & -4.332071 & 2.129762 & -1.555507 \\ 66 & 1 & 0 & -4.410310 & 2.520374 & 0.178056\end{array}$

(16) $\mathrm{TS}_{5}$
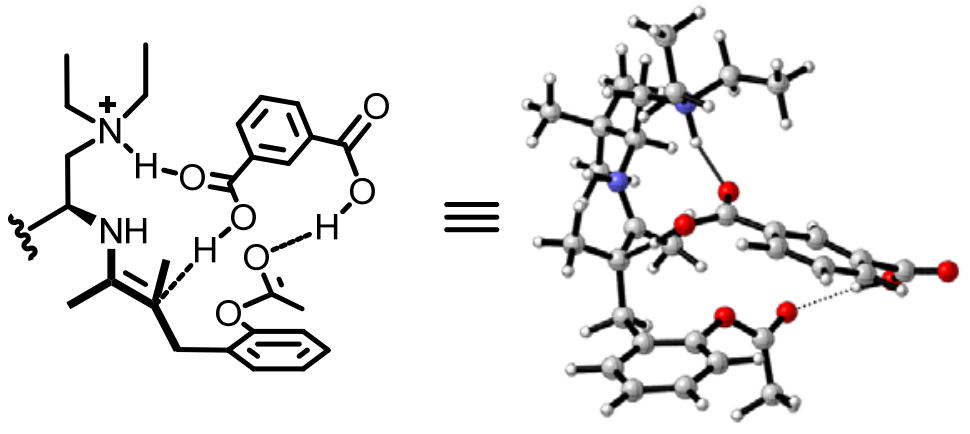

$\mathrm{E}_{\mathrm{sol}}=-1768.942138$

Zero-point correction=

0.729706 (Hartree/Particle)

Thermal correction to Energy=

0.770400

Thermal correction to Enthalpy=

0.771345

Thermal correction to Gibbs Free Energy=

0. 656281

Sum of electronic and zero-point Energies=

$-1767.590262$

Sum of electronic and thermal Energies=

$-1767.549567$

Sum of electronic and thermal Enthalpies=

$-1767.548623$

Sum of electronic and thermal Free Energies=

$-1767.663687$

$\begin{array}{lccc} & \text { E (Thermal) } & \text { CV } & \text { S } \\ & \text { KCal/Mol } & \text { Cal/Mol-Kelvin } & \text { Cal/Mo1-Kelvin } \\ \text { Total } & 483.434 & 156.072 & 242.172 \\ \text { Electronic } & 0.000 & 0.000 & 0.000 \\ \text { Translational } & 0.889 & 2.981 & 44.752\end{array}$


Rotational

Vibrational

481. 656

2. 981

37.565

Standard orientation:

\begin{tabular}{|c|c|c|c|c|c|}
\hline \multirow{2}{*}{$\begin{array}{l}\text { Center } \\
\text { Number }\end{array}$} & \multirow{2}{*}{$\begin{array}{l}\text { Atomic } \\
\text { Number }\end{array}$} & \multirow{2}{*}{$\begin{array}{l}\text { Atomic } \\
\text { Type }\end{array}$} & \multicolumn{3}{|c|}{ Coordinates (Angstroms) } \\
\hline & & & X & Y & Z \\
\hline 1 & 6 & 0 & 5. 023503 & 0.304090 & -1.339465 \\
\hline 2 & 6 & 0 & 3. 718092 & -0.140608 & -0.625258 \\
\hline 3 & 1 & 0 & 3. 134906 & -0.740718 & -1.330983 \\
\hline 4 & 6 & 0 & 3. 962153 & -0.985067 & 0.632426 \\
\hline 5 & 1 & 0 & 4. 269393 & -0.344013 & 1. 464543 \\
\hline 6 & 1 & 0 & 4. 752395 & -1.720872 & 0.475186 \\
\hline 7 & 7 & 0 & 2.892285 & 0.993942 & -0.210906 \\
\hline 8 & 1 & 0 & 1.867587 & -1.250633 & 0.579091 \\
\hline 9 & 7 & 0 & 2.718456 & -1.714894 & 1. 039337 \\
\hline 10 & 6 & 0 & 2.438624 & -1.631810 & 2.512321 \\
\hline 11 & 1 & 0 & 2. 404451 & -0.563652 & 2. 745157 \\
\hline 12 & 1 & 0 & 1. 427901 & -2.019662 & 2. 658921 \\
\hline 13 & 6 & 0 & 2. 728415 & -3.120409 & 0.497157 \\
\hline 14 & 1 & 0 & 2. 843253 & -3.013006 & -0.586032 \\
\hline 15 & 1 & 0 & 3. 626577 & -3.609024 & 0.885453 \\
\hline 16 & 6 & 0 & 1. 471773 & -3.906189 & 0.827697 \\
\hline 17 & 1 & 0 & 1. 402774 & -4.142207 & 1.892825 \\
\hline 18 & 1 & 0 & 0.576254 & -3.364515 & 0.513690 \\
\hline 19 & 1 & 0 & 1. 507760 & -4.853863 & 0.285141 \\
\hline 20 & 6 & 0 & 3. 454490 & -2.375780 & 3. 359616 \\
\hline 21 & 1 & 0 & 4. 477931 & -2.038406 & 3. 168249 \\
\hline 22 & 1 & 0 & 3. 238438 & -2.192444 & 4. 414395 \\
\hline 23 & 1 & 0 & 3. 406970 & -3.455737 & 3. 196593 \\
\hline 24 & 1 & 0 & 3. 235792 & 1. 480008 & 0.610391 \\
\hline 25 & 6 & 0 & 1. 742631 & 1. 463610 & -0.708618 \\
\hline 26 & 6 & 0 & 0.982955 & 2. 357343 & 0.084752 \\
\hline 27 & 6 & 0 & 1. 246274 & 0.983622 & -2.039646 \\
\hline 28 & 1 & 0 & 1. 737224 & 0.073716 & -2.377690 \\
\hline 29 & 1 & 0 & 0.174748 & 0.782652 & -1.989359 \\
\hline 30 & 1 & 0 & 1. 431592 & 1. 767193 & -2.783905 \\
\hline 31 & 6 & 0 & 1. 635074 & 2. 985966 & 1. 309934 \\
\hline 32 & 1 & 0 & 2. 464000 & 3. 656962 & 1. 047569 \\
\hline 33 & 1 & 0 & 0.887566 & 3. 572344 & 1. 850169 \\
\hline 34 & 1 & 0 & 1. 995111 & 2. 235067 & 2. 024180 \\
\hline 35 & 6 & 0 & -0.056759 & 3. 262065 & -0.588613 \\
\hline 36 & 1 & 0 & -0.247605 & 2. 940668 & -1.615122 \\
\hline 37 & 1 & 0 & 0.353660 & 4. 277874 & -0.648230 \\
\hline
\end{tabular}




\begin{tabular}{|c|c|c|c|c|c|}
\hline 38 & 6 & 0 & -1.357981 & 3. 301214 & 0.177179 \\
\hline 39 & 6 & 0 & -1.682373 & 4. 333306 & 1. 056250 \\
\hline 40 & 6 & 0 & -2.263947 & 2. 245385 & 0.059343 \\
\hline 41 & 6 & 0 & -2.857975 & 4. 300013 & 1. 803402 \\
\hline 42 & 1 & 0 & -1.004267 & 5. 178244 & 1. 149163 \\
\hline 43 & 6 & 0 & -3.425596 & 2. 174084 & 0.814631 \\
\hline 44 & 6 & 0 & -3.723893 & 3. 217066 & 1.688765 \\
\hline 45 & 1 & 0 & -3.093075 & 5. 116163 & 2. 478229 \\
\hline 46 & 1 & 0 & -4.074267 & 1. 307049 & 0.730976 \\
\hline 47 & 1 & 0 & -4.632696 & 3. 178193 & 2. 279945 \\
\hline 48 & 8 & 0 & -1.897510 & 1. 211961 & -0.800143 \\
\hline 49 & 6 & 0 & -2.665029 & 0.808063 & -1.847676 \\
\hline 50 & 6 & 0 & -3.899248 & 1. 594706 & -2.197243 \\
\hline 51 & 1 & 0 & -3.752780 & 2. 667506 & -2.058743 \\
\hline 52 & 1 & 0 & -4.149693 & 1. 367626 & -3.232834 \\
\hline 53 & 1 & 0 & -4.734127 & 1. 284909 & -1.562179 \\
\hline 54 & 8 & 0 & -2.287569 & -0.162199 & -2.458590 \\
\hline 55 & 6 & 0 & 5. 911203 & 1. 127921 & -0.399190 \\
\hline 56 & 1 & 0 & 6.815520 & 1. 443211 & -0.927969 \\
\hline 57 & 1 & 0 & 5. 407211 & 2. 038977 & -0.055752 \\
\hline 58 & 1 & 0 & 6.235201 & 0.553385 & 0.476242 \\
\hline 59 & 6 & 0 & 4. 644354 & 1. 152151 & -2.559543 \\
\hline 60 & 1 & 0 & 4. 030648 & 0.581245 & -3.265903 \\
\hline 61 & 1 & 0 & 4. 096869 & 2. 055620 & -2.272719 \\
\hline 62 & 1 & 0 & 5. 549744 & 1. 466908 & -3.086300 \\
\hline 63 & 6 & 0 & 5. 786312 & -0.936116 & -1.825578 \\
\hline 64 & 1 & 0 & 6. 603889 & -0.628984 & -2.484010 \\
\hline 65 & 1 & 0 & 6. 237405 & -1.505515 & -1.006894 \\
\hline 66 & 1 & 0 & 5. 136420 & -1.606432 & -2.400802 \\
\hline 67 & 6 & 0 & -0.403472 & -0.565157 & 0.788180 \\
\hline 68 & 8 & 0 & -0.158942 & 0.459754 & 1.514072 \\
\hline 69 & 1 & 0 & 0.378437 & 1. 343401 & 0.718441 \\
\hline 70 & 8 & 0 & 0.426411 & -1.036749 & -0.032777 \\
\hline 71 & 6 & 0 & -1.750714 & -1.191481 & 0.900255 \\
\hline 72 & 6 & 0 & -2.205538 & -1.964252 & -0.166707 \\
\hline 73 & 6 & 0 & -2.588983 & -0.922908 & 1. 982859 \\
\hline 74 & 6 & 0 & -3.512523 & -2.438657 & -0.171922 \\
\hline 75 & 1 & 0 & -1.536384 & -2.140548 & -1.002577 \\
\hline 76 & 6 & 0 & -3.871283 & -1.468942 & 2. 013330 \\
\hline 77 & 1 & 0 & -2.230716 & -0.282956 & 2. 783337 \\
\hline 78 & 6 & 0 & -4.334019 & -2.222389 & 0.937490 \\
\hline 79 & 1 & 0 & -4.517545 & -1.289345 & 2. 866651 \\
\hline 80 & 1 & 0 & -5.335817 & -2.641491 & 0.92949 \\
\hline 81 & 6 & 0 & -4.067295 & -3.184294 & -1.352677 \\
\hline
\end{tabular}




$\begin{array}{llllll}82 & 8 & 0 & -4.923694 & -4.019585 & -1.244700 \\ 83 & 8 & 0 & -3.546175 & -2.857937 & -2.548420 \\ 84 & 1 & 0 & -3.049380 & -2.019490 & -2.511302\end{array}$

(17) TS6

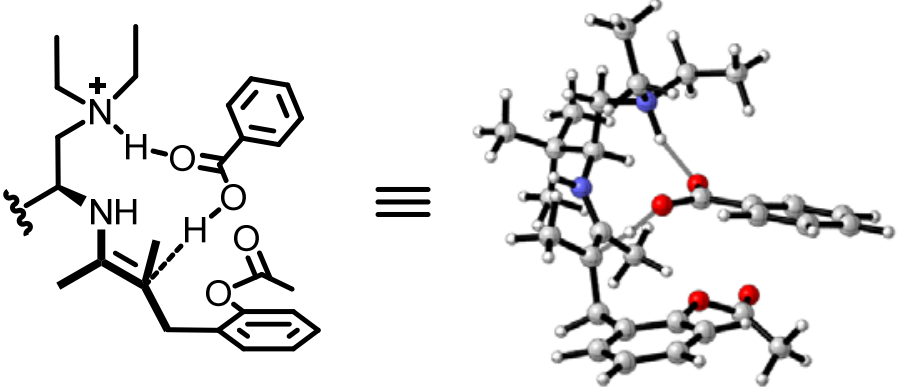

$\mathrm{E}_{\mathrm{sol}}=-1580.360747$

Zero-point correction=

0.714921 (Hartree/Particle)

Thermal correction to Energy=

0.752345

Thermal correction to Enthalpy=

0.753290

Thermal correction to Gibbs Free Energy=

0.646728

Sum of electronic and zero-point Energies=

$-1579.103772$

Sum of electronic and thermal Energies=

Sum of electronic and thermal Enthalpies=

$-1579.066348$

$-1579.065404$

Sum of electronic and thermal Free Energies=

$-1579.171965$

\begin{tabular}{lrcr} 
& E (Thermal) & CV & \multicolumn{1}{c}{ S } \\
& KCal/Mol & Cal/Mol-Kelvin & Cal/Mo1-Kelvin \\
Total & 472.104 & 145.928 & 224.277 \\
Electronic & 0.000 & 0.000 & 0.000 \\
Translational & 0.889 & 2.981 & 44.500 \\
Rotational & 0.889 & 2.981 & 36.984 \\
Vibrational & 470.326 & 139.967 & 142.793
\end{tabular}

Standard orientation:

$\begin{array}{lllll}\text { Center } & \text { Atomic } & \text { Atomic } & \text { Coordinates (Angstroms) } \\ \text { Number } & \text { Number } & \text { Type } & \mathrm{X} & \mathrm{Y} \\ \mathrm{Z}\end{array}$

$\begin{array}{rrrrrr}1 & 6 & 0 & 4.695411 & -1.155350 & 0.844170 \\ 2 & 6 & 0 & 3.421189 & -0.367943 & 0.428918 \\ 3 & 1 & 0 & 3.018776 & 0.124870 & 1.320104 \\ 4 & 6 & 0 & 3.695402 & 0.706532 & -0.630292 \\ 5 & 1 & 0 & 3.852033 & 0.239471 & -1.607182 \\ 6 & 1 & 0 & 4.592938 & 1.279281 & -0.392345 \\ 7 & 7 & 0 & 2.374297 & -1.230903 & -0.116620 \\ 8 & 1 & 0 & 1.691652 & 1.256346 & -0.233710 \\ 9 & 7 & 0 & 2.546057 & 1.661533 & -0.748040\end{array}$




\begin{tabular}{|c|c|c|c|c|c|}
\hline 10 & 6 & 0 & 2. 082427 & 1.854715 & -2.163085 \\
\hline 11 & 1 & 0 & 1. 861566 & 0.852157 & -2.539489 \\
\hline 12 & 1 & 0 & 1. 128206 & 2. 383747 & -2.108595 \\
\hline 13 & 6 & 0 & 2. 846890 & 2. 946043 & -0.022507 \\
\hline 14 & 1 & 0 & 3. 049017 & 2. 652063 & 1. 012200 \\
\hline 15 & 1 & 0 & 3. 770581 & 3. 349689 & -0.446858 \\
\hline 16 & 6 & 0 & 1. 715094 & 3. 956650 & -0.080742 \\
\hline 17 & 1 & 0 & 1.568668 & 4. 353824 & -1.088634 \\
\hline 18 & 1 & 0 & 0.780239 & 3. 520547 & 0.278746 \\
\hline 19 & 1 & 0 & 1.973804 & 4. 797433 & 0.567399 \\
\hline 20 & 6 & 0 & 3. 088706 & 2. 586949 & -3.031800 \\
\hline 21 & 1 & 0 & 4. 060833 & 2. 084414 & -3.051920 \\
\hline 22 & 1 & 0 & 2. 713391 & 2. 619361 & -4.057040 \\
\hline 23 & 1 & 0 & 3. 238311 & 3. 618909 & -2.703310 \\
\hline 24 & 1 & 0 & 2. 539950 & -1.540126 & -1.068277 \\
\hline 25 & 6 & 0 & 1. 221997 & -1.646474 & 0.426800 \\
\hline 26 & 6 & 0 & 0.232449 & -2.183294 & -0.430858 \\
\hline 27 & 6 & 0 & 0.970487 & -1.458289 & 1.891197 \\
\hline 28 & 1 & 0 & -0.045772 & -1.096317 & 2. 061656 \\
\hline 29 & 1 & 0 & 1. 095741 & -2.426823 & 2. 390264 \\
\hline 30 & 1 & 0 & 1. 651508 & -0.747390 & 2.354107 \\
\hline 31 & 6 & 0 & 0.621558 & -2.556653 & -1.855257 \\
\hline 32 & 1 & 0 & 1. 354431 & -3.374126 & -1.891053 \\
\hline 33 & 1 & 0 & -0.270694 & -2.884268 & -2.394835 \\
\hline 34 & 1 & 0 & 1. 013718 & -1.700750 & -2.419012 \\
\hline 35 & 6 & 0 & -0.878099 & -3.064626 & 0.153672 \\
\hline 36 & 1 & 0 & -0.913519 & -2.974645 & 1. 241426 \\
\hline 37 & 1 & 0 & -0.644998 & -4.112641 & -0.073466 \\
\hline 38 & 6 & 0 & -2.232712 & -2.726983 & -0.424016 \\
\hline 39 & 6 & 0 & -2.836967 & -3.494813 & -1.415665 \\
\hline 40 & 6 & 0 & -2.892108 & -1.569304 & 0.008381 \\
\hline 41 & 6 & 0 & -4.052720 & -3.112918 & -1.980580 \\
\hline 42 & 1 & 0 & -2.347762 & -4.408623 & -1.744763 \\
\hline 43 & 6 & 0 & -4.082631 & -1.149966 & -0.571603 \\
\hline 44 & 6 & 0 & -4.664588 & -1.936856 & -1.565066 \\
\hline 45 & 1 & 0 & -4.512104 & -3.727008 & -2.747819 \\
\hline 46 & 1 & 0 & -4.542138 & -0.215980 & -0.273272 \\
\hline 47 & 1 & 0 & -5.600155 & -1.618578 & -2.013368 \\
\hline 48 & 8 & 0 & -2.198976 & -0.859823 & 0.976212 \\
\hline 49 & 6 & 0 & -2.686396 & -0.266495 & 2. 110273 \\
\hline 50 & 6 & 0 & -4.169049 & -0.109698 & 2. 291910 \\
\hline 51 & 1 & 0 & -4.727705 & -0.993403 & 1. 979767 \\
\hline 52 & 1 & 0 & -4.348238 & 0.105253 & 3. 344480 \\
\hline 53 & 1 & 0 & -4.503763 & 0.748481 & 1.69718 \\
\hline
\end{tabular}




\begin{tabular}{|c|c|c|c|c|c|}
\hline 54 & 8 & 0 & -1.867082 & 0.131012 & 2. 893325 \\
\hline 55 & 6 & 0 & 5. 347667 & -1.822282 & -0.372850 \\
\hline 56 & 1 & 0 & 6.216729 & -2.404987 & -0.053800 \\
\hline 57 & 1 & 0 & 4. 661379 & -2.516911 & -0.871507 \\
\hline 58 & 1 & 0 & 5. 703293 & -1.090363 & -1.106833 \\
\hline 59 & 6 & 0 & 4. 298704 & -2.231586 & 1. 861410 \\
\hline 60 & 1 & 0 & 3. 863175 & -1.788730 & 2. 764145 \\
\hline 61 & 1 & 0 & 3. 580202 & -2.942422 & 1. 441524 \\
\hline 62 & 1 & 0 & 5. 185480 & -2.796041 & 2. 164331 \\
\hline 63 & 6 & 0 & 5. 691199 & -0.197925 & 1. 513319 \\
\hline 64 & 1 & 0 & 6.513715 & -0.771201 & 1. 950738 \\
\hline 65 & 1 & 0 & 6. 138606 & 0.511658 & 0.809963 \\
\hline 66 & 1 & 0 & 5. 216632 & 0.367408 & 2. 324157 \\
\hline 67 & 6 & 0 & -0.631032 & 1. 041059 & -0.223066 \\
\hline 68 & 8 & 0 & -0.635016 & 0. 192719 & -1.183311 \\
\hline 69 & 1 & 0 & -0.227740 & -0.950048 & -0.690928 \\
\hline 70 & 8 & 0 & 0.338319 & 1. 172267 & 0.568751 \\
\hline 71 & 6 & 0 & -2.837010 & 1. 912978 & -1.029101 \\
\hline 72 & 6 & 0 & -1.832388 & 1. 912796 & -0.058537 \\
\hline 73 & 6 & 0 & -1.964767 & 2. 693966 & 1. 093143 \\
\hline 74 & 6 & 0 & -3.103212 & 3. 472597 & 1. 273454 \\
\hline 75 & 6 & 0 & -4.102081 & 3. 480945 & 0.299607 \\
\hline 76 & 6 & 0 & -3.968614 & 2. 703757 & -0.851235 \\
\hline 77 & 1 & 0 & -2.720564 & 1. 282957 & -1.905414 \\
\hline 78 & 1 & 0 & -1.188547 & 2. 646513 & 1. 850385 \\
\hline 79 & 1 & 0 & -3.214766 & 4. 069471 & 2. 173072 \\
\hline 80 & 1 & 0 & -4.987874 & 4. 093605 & 0.438331 \\
\hline 81 & 1 & 0 & -4.747498 & 2. 714913 & -1.607700 \\
\hline
\end{tabular}

(18) $\mathrm{TS}_{7}$

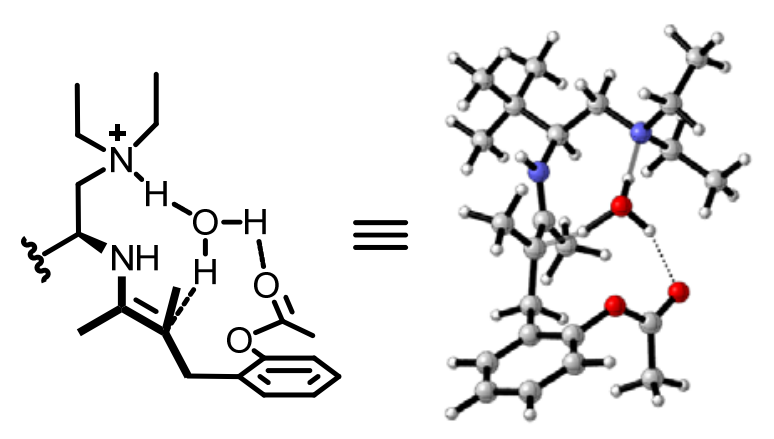

$E_{\text {sol }}=-1235.987856$

Zero-point correction=

0. 620465 (Hartree/Particle)

Thermal correction to Energy=

0.652439

Thermal correction to Enthalpy=

0.653383

Thermal correction to Gibbs Free Energy=

0. 558149 
Sum of electronic and zero-point Energies=

Sum of electronic and thermal Energies=

Sum of electronic and thermal Enthalpies=

Sum of electronic and thermal Free Energies=
$-1234.922271$

$-1234.890298$

$-1234.889353$

$-1234.984587$
E (Therma1)

$\mathrm{KCa} 1 / \mathrm{Mol}$

Total 409. 412

Electronic

Translational

Rotational

Vibrational

Standard orientation:

\begin{tabular}{|c|c|c|c|c|c|}
\hline \multirow{2}{*}{$\begin{array}{l}\text { Center } \\
\text { Number }\end{array}$} & \multirow{2}{*}{$\begin{array}{l}\text { Atomic } \\
\text { Number }\end{array}$} & \multirow{2}{*}{$\begin{array}{c}\text { Atomic } \\
\text { Type }\end{array}$} & \multicolumn{3}{|c|}{ Coordinates (Angstroms) } \\
\hline & & & $\mathrm{X}$ & Y & Z \\
\hline 1 & 6 & 0 & 3. 786651 & -2.127809 & 0.182770 \\
\hline 2 & 6 & 0 & 2. 862680 & -0.880902 & 0.275075 \\
\hline 3 & 1 & 0 & 2. 883392 & -0.557166 & 1. 316597 \\
\hline 4 & 6 & 0 & 3. 291313 & 0.299613 & -0.609151 \\
\hline 5 & 1 & 0 & 3.221838 & 0.003494 & -1.662877 \\
\hline 6 & 1 & 0 & 4. 344737 & 0.531310 & -0.404289 \\
\hline 7 & 7 & 0 & 1. 479937 & -1.237698 & -0.057931 \\
\hline 8 & 1 & 0 & 0.872140 & 1. 298712 & -0.620936 \\
\hline 9 & 7 & 0 & 2.458915 & 1.503528 & -0.430127 \\
\hline 10 & 6 & 0 & 2. 634312 & 2. 435330 & -1.574480 \\
\hline 11 & 1 & 0 & 2. 381768 & 1. 867120 & -2.475715 \\
\hline 12 & 1 & 0 & 1.871037 & 3. 213242 & -1.481873 \\
\hline 13 & 6 & 0 & 2. 731317 & 2. 146790 & 0.872970 \\
\hline 14 & 1 & 0 & 2. 520609 & 1. 409379 & 1. 653897 \\
\hline 15 & 1 & 0 & 3. 800286 & 2. 394337 & 0.954811 \\
\hline 16 & 6 & 0 & 1.878715 & 3. 381919 & 1. 129790 \\
\hline 17 & 1 & 0 & 2. 137269 & 4. 210067 & 0.465116 \\
\hline 18 & 1 & 0 & 0.810757 & 3. 165988 & 1. 017870 \\
\hline 19 & 1 & 0 & 2. 040930 & 3. 724641 & 2. 154648 \\
\hline 20 & 6 & 0 & 4. 014883 & 3. 067844 & -1.713623 \\
\hline 21 & 1 & 0 & 4. 801496 & 2. 316709 & -1.831146 \\
\hline 22 & 1 & 0 & 4. 031795 & 3. 702699 & -2.602993 \\
\hline 23 & 1 & 0 & 4. 265542 & 3. 698131 & -0.855351 \\
\hline 24 & 1 & 0 & 1. 356669 & -1.548039 & -1.016583 \\
\hline 25 & 6 & 0 & 0.357353 & -1.106046 & 0.653132 \\
\hline 26 & 6 & 0 & -0.889812 & -1.154043 & -0.003877 \\
\hline 27 & 6 & 0 & 0.448077 & -0.716280 & 2. 103407 \\
\hline
\end{tabular}




\begin{tabular}{|c|c|c|c|c|c|}
\hline 28 & 1 & 0 & 0.208931 & 0.349152 & 2. 217791 \\
\hline 29 & 1 & 0 & -0.277285 & -1.284591 & 2. 688184 \\
\hline 30 & 1 & 0 & 1. 432306 & -0.902262 & 2. 529602 \\
\hline 31 & 6 & 0 & -0.940457 & -1.785664 & -1.389497 \\
\hline 32 & 1 & 0 & -0.580149 & -2.823167 & -1.386295 \\
\hline 33 & 1 & 0 & -1.966191 & -1.794358 & -1.762233 \\
\hline 34 & 1 & 0 & -0.355967 & -1.216141 & -2.122722 \\
\hline 35 & 6 & 0 & -2.146322 & -1.282094 & 0.866828 \\
\hline 36 & 1 & 0 & -2.092949 & -0.558908 & 1. 691103 \\
\hline 37 & 1 & 0 & -2.178567 & -2.273954 & 1. 337709 \\
\hline 38 & 6 & 0 & -3.455924 & -1.083511 & 0.136764 \\
\hline 39 & 6 & 0 & -4.381471 & -2.117904 & -0.007343 \\
\hline 40 & 6 & 0 & -3.798698 & 0.153870 & -0.410753 \\
\hline 41 & 6 & 0 & -5.590400 & -1.918691 & -0.670992 \\
\hline 42 & 1 & 0 & -4.148075 & -3.094672 & 0.408161 \\
\hline 43 & 6 & 0 & -4.988762 & 0.376928 & -1.084481 \\
\hline 44 & 6 & 0 & -5.895249 & -0.672929 & -1.211015 \\
\hline 45 & 1 & 0 & -6.292697 & -2.739636 & -0.768771 \\
\hline 46 & 1 & 0 & -5.185147 & 1. 356869 & -1.507996 \\
\hline 47 & 1 & 0 & -6.832861 & -0.515531 & -1.733165 \\
\hline 48 & 8 & 0 & -2.856144 & 1. 181467 & -0.337308 \\
\hline 49 & 6 & 0 & -2.754574 & 1. 967249 & 0.758461 \\
\hline 50 & 6 & 0 & -3.890738 & 1. 970946 & 1. 739521 \\
\hline 51 & 1 & 0 & -4.039637 & 0.972556 & 2. 161541 \\
\hline 52 & 1 & 0 & -3.665010 & 2. 684280 & 2. 529984 \\
\hline 53 & 1 & 0 & -4.820185 & 2. 247981 & 1. 234038 \\
\hline 54 & 8 & 0 & -1.748186 & 2. 633545 & 0.862969 \\
\hline 55 & 6 & 0 & 3. 738795 & -2.771689 & -1.208752 \\
\hline 56 & 1 & 0 & 4. 425210 & -3.623247 & -1.241057 \\
\hline 57 & 1 & 0 & 2. 743061 & -3.162485 & -1.450394 \\
\hline 58 & 1 & 0 & 4. 047200 & -2.079177 & -1.999231 \\
\hline 59 & 6 & 0 & 3. 313851 & -3.144868 & 1. 229106 \\
\hline 60 & 1 & 0 & 3. 396627 & -2.734320 & 2. 242743 \\
\hline 61 & 1 & 0 & 2. 275071 & -3.446588 & 1. 060116 \\
\hline 62 & 1 & 0 & 3. 933799 & -4.045214 & 1. 186696 \\
\hline 63 & 6 & 0 & 5. 231526 & -1.724968 & 0.508885 \\
\hline 64 & 1 & 0 & 5. 838362 & -2.623552 & 0.655217 \\
\hline 65 & 1 & 0 & 5.695253 & -1.148627 & -0.297018 \\
\hline 66 & 1 & 0 & 5. 285324 & -1.135464 & 1. 432107 \\
\hline 67 & 8 & 0 & -0.127586 & 1. 221317 & -0.886248 \\
\hline 68 & 1 & 0 & -0.604586 & 0.176770 & -0.376509 \\
\hline 69 & 1 & 0 & -0.641134 & 1. 953669 & -0.476139 \\
\hline
\end{tabular}

\section{(19) Int7}



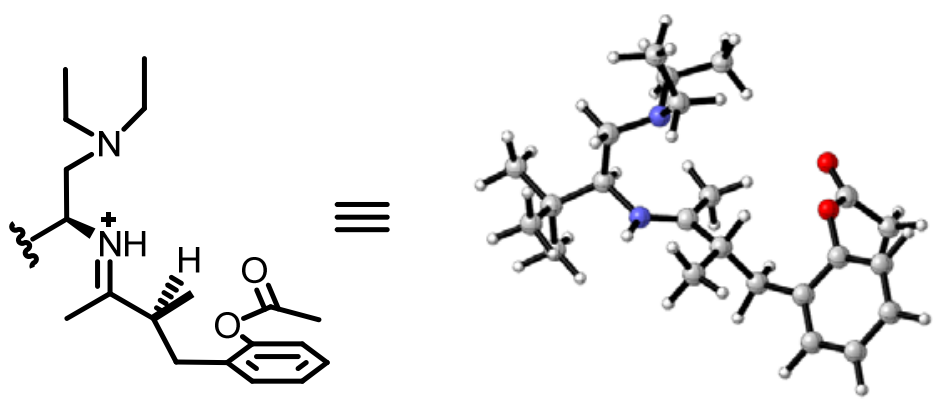

$\mathrm{E}_{\mathrm{sol}}=-1159.576987$

Zero-point correction=

0.599556 (Hartree/Particle)

Thermal correction to Energy=

0.629852

Thermal correction to Enthalpy=

0.630796

Thermal correction to Gibbs Free Energy=

0.537702

Sum of electronic and zero-point Energies=

$-1158.566982$

Sum of electronic and thermal Energies=

$-1158.536687$

Sum of electronic and thermal Enthalpies=

$-1158.535742$

Sum of electronic and thermal Free Energies=

$-1158.628836$

E (Therma1)

CV

S

KCa1/Mol

Total

395. 238

Electronic

0.000

Translational

0. 889

Rotational

0.889

393. 460
Cal/Mo1-Kelvin

116. 031

0. 000

2. 981

2. 981

110. 070
Cal/Mol-Kelvin

195. 932

0.000

43. 660

35. 948

116. 324

Standard orientation:

\begin{tabular}{rrrrrr} 
Center & Atomic & Atomic & \multicolumn{3}{c}{ Coordinates Angstroms) } \\
Number & Number & Type & X & Y & $Z$ \\
-1 & 6 & 0 & -4.083303 & -1.711461 & -0.507211 \\
1 & 6 & 0 & -3.052157 & -0.560717 & -0.456489 \\
2 & 1 & 0 & -3.022524 & -0.096322 & -1.445774 \\
3 & 6 & 0 & -3.289886 & 0.535620 & 0.612152 \\
4 & 1 & 0 & -3.321231 & 0.059644 & 1.599430 \\
5 & 1 & 0 & -4.279691 & 0.979765 & 0.440776 \\
6 & 7 & 0 & -1.707017 & -1.083071 & -0.171411 \\
7 & 7 & 0 & -2.226926 & 1.526220 & 0.575520 \\
8 & 6 & 0 & -1.681413 & 1.857233 & 1.896778 \\
9 & 1 & 0 & -1.409476 & 0.907721 & 2.377013 \\
10 & 1 & 0 & -0.744047 & 2.402463 & 1.746657 \\
11 & 6 & 0 & -2.598628 & 2.689506 & -0.238359 \\
12 & 1 & 0 & -2.904835 & 2.310504 & -1.223784 \\
13 & 1 & 0 & -3.484723 & 3.198274 & 0.176540 \\
14 & & 0 & & &
\end{tabular}




\begin{tabular}{|c|c|c|c|c|c|}
\hline 15 & 6 & 0 & -1.462975 & 3. 688918 & -0.412027 \\
\hline 16 & 1 & 0 & -1.254486 & 4. 227228 & 0.516945 \\
\hline 17 & 1 & 0 & -0.538653 & 3. 200032 & -0.736274 \\
\hline 18 & 1 & 0 & -1.739020 & 4. 432894 & -1.163327 \\
\hline 19 & 6 & 0 & -2.613179 & 2. 651725 & 2. 810464 \\
\hline 20 & 1 & 0 & -3.571617 & 2. 139007 & 2. 947012 \\
\hline 21 & 1 & 0 & -2.159342 & 2. 783429 & 3. 796205 \\
\hline 22 & 1 & 0 & -2.817326 & 3. 646816 & 2. 403880 \\
\hline 23 & 1 & 0 & -1.682689 & -1.762333 & 0.587436 \\
\hline 24 & 6 & 0 & -0.568317 & -0.587998 & -0.535994 \\
\hline 25 & 6 & 0 & 0.678201 & -1.000298 & 0.203845 \\
\hline 26 & 6 & 0 & -0.434796 & 0.371438 & -1.667666 \\
\hline 27 & 1 & 0 & 0.356586 & 1. 095848 & -1.449266 \\
\hline 28 & 1 & 0 & -0.133503 & -0.193388 & -2.559728 \\
\hline 29 & 1 & 0 & -1.352087 & 0.909841 & -1.887353 \\
\hline 30 & 6 & 0 & 0.468969 & -2.059723 & 1. 283576 \\
\hline 31 & 1 & 0 & 0.106259 & -3.004568 & 0.859966 \\
\hline 32 & 1 & 0 & 1.425113 & -2.267457 & 1. 769436 \\
\hline 33 & 1 & 0 & -0.218659 & -1.727971 & 2. 069395 \\
\hline 34 & 6 & 0 & 1. 844716 & -1.389500 & -0.742929 \\
\hline 35 & 1 & 0 & 1.842841 & -0.748736 & -1.630458 \\
\hline 36 & 1 & 0 & 1. 697611 & -2.418392 & -1.090477 \\
\hline 37 & 6 & 0 & 3. 175364 & -1.268416 & -0.039838 \\
\hline 38 & 6 & 0 & 3. 942246 & -2.373584 & 0.327970 \\
\hline 39 & 6 & 0 & 3. 659261 & 0.000879 & 0.293058 \\
\hline 40 & 6 & 0 & 5. 147582 & -2.212209 & 1. 008736 \\
\hline 41 & 1 & 0 & 3. 591045 & -3.370747 & 0.074544 \\
\hline 42 & 6 & 0 & 4. 845677 & 0.181602 & 0.987114 \\
\hline 43 & 6 & 0 & 5.596340 & -0.937357 & 1. 341931 \\
\hline 44 & 1 & 0 & 5. 732361 & -3.083502 & 1. 283323 \\
\hline 45 & 1 & 0 & 5. 163875 & 1. 186131 & 1. 247975 \\
\hline 46 & 1 & 0 & 6.529405 & -0.809776 & 1. 880369 \\
\hline 47 & 8 & 0 & 2. 857314 & 1. 091811 & -0.031484 \\
\hline 48 & 6 & 0 & 3. 028688 & 1. 768120 & -1.213208 \\
\hline 49 & 6 & 0 & 4. 330455 & 1. 570223 & -1.940363 \\
\hline 50 & 1 & 0 & 4. 476743 & 0.516021 & -2.194366 \\
\hline 51 & 1 & 0 & 4. 308906 & 2. 174409 & -2.845453 \\
\hline 52 & 1 & 0 & 5. 171951 & 1. 870928 & -1.310662 \\
\hline 53 & 8 & 0 & 2. 128885 & 2. 471344 & -1.583349 \\
\hline 54 & 6 & 0 & -4.139435 & -2.506759 & 0.805198 \\
\hline 55 & 1 & 0 & -4.912368 & -3.276786 & 0.724471 \\
\hline 56 & 1 & 0 & -3.204711 & -3.040123 & 1. 025489 \\
\hline 57 & 1 & 0 & -4.395440 & -1.880444 & 1. 665020 \\
\hline 58 & 6 & 0 & -3.704455 & -2.653011 & -1.6581 \\
\hline
\end{tabular}




\begin{tabular}{rrrrrr}
59 & 1 & 0 & -3.688531 & -2.119638 & -2.615214 \\
60 & 1 & 0 & -2.719891 & -3.109272 & -1.505362 \\
61 & 1 & 0 & -4.435400 & -3.462824 & -1.739409 \\
62 & 6 & 0 & -5.466054 & -1.108453 & -0.789830 \\
63 & 1 & 0 & -6.180127 & -1.909839 & -1.000612 \\
64 & 1 & 0 & -5.851051 & -0.541804 & 0.062977 \\
65 & 1 & 0 & -5.441421 & -0.446637 & -1.663082 \\
66 & 1 & 0 & 0.970752 & -0.052343 & 0.683051 \\
\hline
\end{tabular}

\section{(20) TS8}
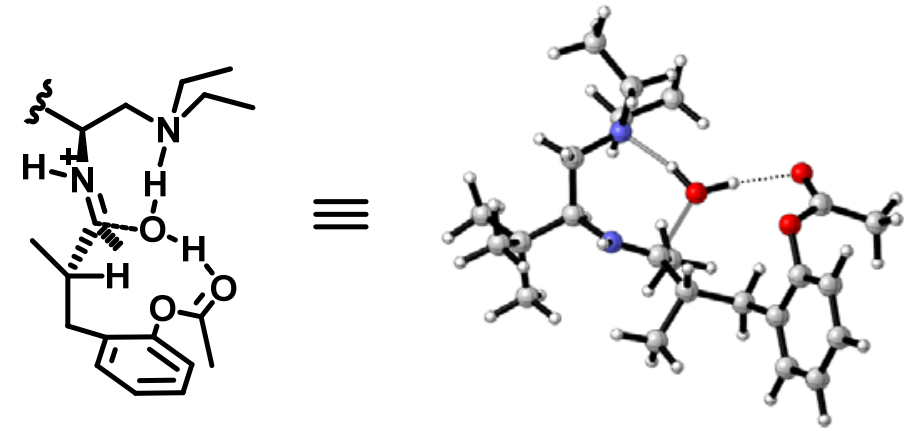

$\mathrm{E}_{\mathrm{sol}}=-1236.00794$

Zero-point correction=

0.625755 (Hartree/Particle)

Thermal correction to Energy=

0.657104

Thermal correction to Enthalpy=

0.658048

Thermal correction to Gibbs Free Energy=

Sum of electronic and zero-point Energies=

0.564421

Sum of electronic and thermal Energies=

$-1234.939029$

$-1234.907680$

Sum of electronic and thermal Enthalpies=

Sum of electronic and thermal Free Energies=

$-1234.906735$

$-1235.000363$

\section{E (Thermal) \\ KCal/Mol}

Total

412. 339

Electronic

Translational

Rotational

Vibrational

$\mathrm{CV}$ Cal/Mo1-Kelvin 121. 245

0.000

2. 981

2. 981

115. 283

$\mathrm{S}$

Cal/Mo1-Kelvin

197. 055

0.000

43. 800

36. 155

117. 100

Standard orientation:

\begin{tabular}{|c|c|c|c|c|c|}
\hline \multirow{2}{*}{$\begin{array}{l}\text { Center } \\
\text { Number }\end{array}$} & \multirow{2}{*}{$\begin{array}{l}\text { Atomic } \\
\text { Number }\end{array}$} & \multirow{2}{*}{$\begin{array}{l}\text { Atomic } \\
\text { Type }\end{array}$} & \multicolumn{3}{|c|}{ Coordinates (Angstroms) } \\
\hline & & & $X$ & $\mathrm{Y}$ & Z \\
\hline 1 & 6 & 0 & -3.616695 & -2.210347 & 0.152101 \\
\hline 2 & 6 & 0 & -2.819507 & -0.902470 & -0.107286 \\
\hline 3 & 1 & 0 & -2.935454 & -0.682595 & -1.171632 \\
\hline & & & S72 & & \\
\hline
\end{tabular}




\begin{tabular}{|c|c|c|c|c|c|}
\hline 4 & 6 & 0 & -3.283259 & 0.313256 & 0.712550 \\
\hline 5 & 1 & 0 & -3.005874 & 0.156794 & 1. 761745 \\
\hline 6 & 1 & 0 & -4.378526 & 0.381506 & 0.675710 \\
\hline 7 & 7 & 0 & -1.386302 & -1.130316 & 0.128744 \\
\hline 8 & 7 & 0 & -2.668148 & 1. 568406 & 0.257842 \\
\hline 9 & 6 & 0 & -2.536540 & 2. 547787 & 1. 360111 \\
\hline 10 & 1 & 0 & -1.969194 & 2. 046587 & 2. 151622 \\
\hline 11 & 1 & 0 & -1.902124 & 3. 365007 & 1. 002495 \\
\hline 12 & 6 & 0 & -3.370745 & 2. 111238 & -0.918537 \\
\hline 13 & 1 & 0 & -3.432591 & 1. 310187 & -1.664415 \\
\hline 14 & 1 & 0 & -4.409032 & 2. 371476 & -0.663347 \\
\hline 15 & 6 & 0 & -2.654219 & 3. 307874 & -1.528566 \\
\hline 16 & 1 & 0 & -2.709505 & 4. 190865 & -0.886690 \\
\hline 17 & 1 & 0 & -1.597437 & 3. 080567 & -1.708874 \\
\hline 18 & 1 & 0 & -3.114127 & 3. 568316 & -2.484775 \\
\hline 19 & 6 & 0 & -3.845733 & 3. 102772 & 1. 912918 \\
\hline 20 & 1 & 0 & -4.511047 & 2. 306476 & 2. 261760 \\
\hline 21 & 1 & 0 & -3.635979 & 3. 755273 & 2. 764246 \\
\hline 22 & 1 & 0 & -4.382689 & 3. 696731 & 1. 167807 \\
\hline 23 & 1 & 0 & -1.152373 & -1.401712 & 1. 080354 \\
\hline 24 & 6 & 0 & -0.358226 & -0.795048 & -0.636808 \\
\hline 25 & 6 & 0 & 0.991010 & -1.338390 & -0.182704 \\
\hline 26 & 6 & 0 & -0.613796 & -0.533149 & -2.095359 \\
\hline 27 & 1 & 0 & 0.287253 & -0.175096 & -2.589677 \\
\hline 28 & 1 & 0 & -0.927344 & -1.470136 & -2.571231 \\
\hline 29 & 1 & 0 & -1.397428 & 0.212010 & -2.239867 \\
\hline 30 & 6 & 0 & 0.954940 & -2.873417 & -0.170378 \\
\hline 31 & 1 & 0 & 0.728080 & -3.271495 & -1.165925 \\
\hline 32 & 1 & 0 & 1. 932720 & -3.257325 & 0.133486 \\
\hline 33 & 1 & 0 & 0.212371 & -3.264763 & 0.530694 \\
\hline 34 & 6 & 0 & 2. 174205 & -0.838706 & -1.041551 \\
\hline 35 & 1 & 0 & 1. 972446 & 0.180335 & -1.384701 \\
\hline 36 & 1 & 0 & 2. 265751 & -1.466279 & -1.935841 \\
\hline 37 & 6 & 0 & 3. 468479 & -0.869114 & -0.263546 \\
\hline 38 & 6 & 0 & 4. 362877 & -1.938193 & -0.314761 \\
\hline 39 & 6 & 0 & 3.783005 & 0.193228 & 0.589213 \\
\hline 40 & 6 & 0 & 5. 511462 & -1.949898 & 0.475150 \\
\hline 41 & 1 & 0 & 4. 159232 & -2.768184 & -0.986863 \\
\hline 42 & 6 & 0 & 4. 909367 & 0.197016 & 1. 395706 \\
\hline 43 & 6 & 0 & 5. 779653 & -0.889654 & 1. 335940 \\
\hline 44 & 1 & 0 & 6. 194906 & -2.790430 & 0.419610 \\
\hline 45 & 1 & 0 & 5. 092384 & 1. 037617 & 2. 057548 \\
\hline 46 & 1 & 0 & 6. 667696 & -0.901627 & 1. 958659 \\
\hline 47 & 8 & 0 & 2. 877622 & 1. 256183 & 0.634318 \\
\hline
\end{tabular}




\begin{tabular}{rrrrrr}
48 & 6 & 0 & 2.981509 & 2.243965 & -0.292758 \\
49 & 6 & 0 & 4.341783 & 2.552250 & -0.848929 \\
50 & 1 & 0 & 4.697522 & 1.712916 & -1.454708 \\
51 & 1 & 0 & 4.267825 & 3.446068 & -1.465761 \\
52 & 1 & 0 & 5.064282 & 2.701793 & -0.043124 \\
53 & 8 & 0 & 1.969202 & 2.821247 & -0.617157 \\
54 & 6 & 0 & -3.457625 & -2.698151 & 1.597748 \\
55 & 1 & 0 & -4.062228 & -3.597490 & 1.749858 \\
56 & 1 & 0 & -2.422380 & -2.975174 & 1.832463 \\
57 & 1 & 0 & -3.795274 & -1.953768 & 2.326910 \\
58 & 6 & 0 & -3.099984 & -3.287204 & -0.811237 \\
59 & 1 & 0 & -3.252931 & -2.986098 & -1.854766 \\
60 & 1 & 0 & -2.034259 & -3.489039 & -0.661831 \\
61 & 1 & 0 & -3.642208 & -4.224412 & -0.654428 \\
62 & 6 & 0 & -5.103530 & -1.967787 & -0.143985 \\
63 & 1 & 0 & -5.630648 & -2.925861 & -0.178031 \\
64 & 1 & 0 & -5.589355 & -1.358591 & 0.623304 \\
65 & 1 & 0 & -5.242982 & -1.477839 & -1.115082 \\
66 & 1 & 0 & 1.132515 & -0.967409 & 0.838833 \\
67 & 8 & 0 & -0.061045 & 1.029460 & -0.008832 \\
68 & 1 & 0 & 0.577889 & 1.733199 & -0.269448 \\
69 & 1 & 0 & -1.015551 & 1.362697 & -0.009007 \\
\hline------------------------------------------
\end{tabular}

(21) Int8

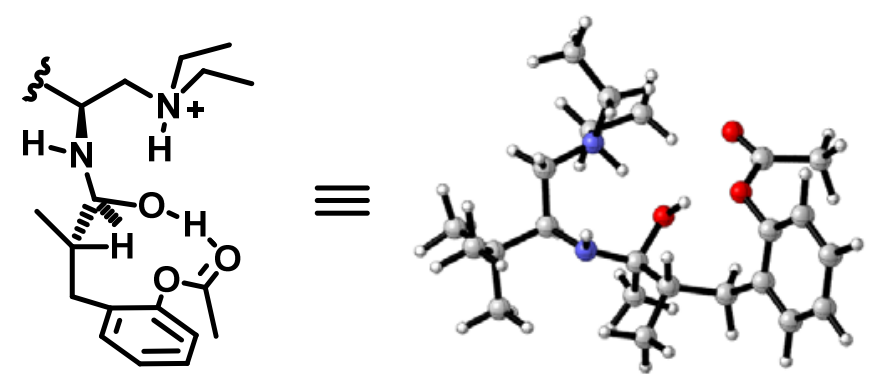

$\mathrm{E}_{\mathrm{sol}}=-1236.032121$

Zero-point correction=

0.629580 (Hartree/Particle)

Thermal correction to Energy=

0.660849

Thermal correction to Enthalpy=

0.661794

Thermal correction to Gibbs Free Energy=

Sum of electronic and zero-point Energies=

0.568389

Sum of electronic and thermal Energies=

$-1234.962839$

$-1234.931569$

$-1234.930625$

Sum of electronic and thermal Enthalpies=

$-1235.024030$

Sum of electronic and thermal Free Energies=

E (Therma1)

$\mathrm{CV}$

$\mathrm{S}$

$\mathrm{KCa} / \mathrm{Mol}$

Cal/Mo1-Kelvin

Cal/Mo1-Kelvin 


$\begin{array}{lrrr}\text { Total } & 414.689 & 121.245 & 196.587 \\ \text { Electronic } & 0.000 & 0.000 & 0.000 \\ \text { Translational } & 0.889 & 2.981 & 43.800 \\ \text { Rotational } & 0.889 & 2.981 & 35.986 \\ \text { Vibrational } & 412.912 & 115.284 & 116.800\end{array}$

Standard orientation:

\begin{tabular}{|c|c|c|c|c|c|}
\hline \multirow{2}{*}{$\begin{array}{l}\text { Center } \\
\text { Number }\end{array}$} & \multirow{2}{*}{$\begin{array}{l}\text { Atomic } \\
\text { Number }\end{array}$} & \multirow{2}{*}{$\begin{array}{c}\text { Atomic } \\
\text { Type }\end{array}$} & \multicolumn{3}{|c|}{ Coordinates (Angstroms) } \\
\hline & & & $X$ & $\mathrm{Y}$ & Z \\
\hline 1 & 6 & 0 & 3. 818289 & -1.824438 & -0.420601 \\
\hline 2 & 6 & 0 & 2.765585 & -0.753128 & -0.009601 \\
\hline 3 & 1 & 0 & 2.907410 & -0.586973 & 1. 066096 \\
\hline 4 & 6 & 0 & 2. 994513 & 0.587538 & -0.733991 \\
\hline 5 & 1 & 0 & 2. 715048 & 0.492651 & -1.787906 \\
\hline 6 & 1 & 0 & 4. 036914 & 0.909234 & -0.689163 \\
\hline 7 & 7 & 0 & 1. 404213 & -1.242110 & -0.202152 \\
\hline 8 & 7 & 0 & 2. 156093 & 1. 682141 & -0.140352 \\
\hline 9 & 6 & 0 & 1. 610444 & 2. 639287 & -1.162993 \\
\hline 10 & 1 & 0 & 1. 109153 & 2. 014231 & -1.907854 \\
\hline 11 & 1 & 0 & 0.835841 & 3. 225861 & -0.665654 \\
\hline 12 & 6 & 0 & 2. 850255 & 2. 349578 & 1. 009821 \\
\hline 13 & 1 & 0 & 3. 174884 & 1. 540999 & 1. 670962 \\
\hline 14 & 1 & 0 & 3. 744469 & 2. 841961 & 0.618473 \\
\hline 15 & 6 & 0 & 1.929965 & 3. 309070 & 1. 744685 \\
\hline 16 & 1 & 0 & 1. 708129 & 4. 201124 & 1. 153958 \\
\hline 17 & 1 & 0 & 0.986757 & 2. 819833 & 2. 006722 \\
\hline 18 & 1 & 0 & 2. 418021 & 3. 633425 & 2. 666423 \\
\hline 19 & 6 & 0 & 2.680916 & 3. 507473 & -1.797989 \\
\hline 20 & 1 & 0 & 3. 487626 & 2. 911519 & -2.235591 \\
\hline 21 & 1 & 0 & 2. 228710 & 4. 092971 & -2.601582 \\
\hline 22 & 1 & 0 & 3. 112326 & 4. 212391 & -1.082668 \\
\hline 23 & 1 & 0 & 1. 070816 & -1.125286 & -1.155242 \\
\hline 24 & 6 & 0 & 0.360950 & -1.038477 & 0.772542 \\
\hline 25 & 6 & 0 & -0.936820 & -1.668563 & 0.220621 \\
\hline 26 & 6 & 0 & 0.749869 & -1.581614 & 2. 140771 \\
\hline 27 & 1 & 0 & -0.114518 & -1.554056 & 2. 809019 \\
\hline 28 & 1 & 0 & 1. 113705 & -2.607430 & 2. 061436 \\
\hline 29 & 1 & 0 & 1. 528280 & -0.965606 & 2. 599744 \\
\hline 30 & 6 & 0 & -0.825379 & -3.174179 & -0.010849 \\
\hline 31 & 1 & 0 & -0.666764 & -3.703734 & 0.934431 \\
\hline 32 & 1 & 0 & -1.755801 & -3.548439 & -0.449553 \\
\hline 33 & 1 & 0 & -0.002339 & -3.421360 & -0.685678 \\
\hline 34 & 6 & 0 & -2.170055 & -1.341165 & 1. 105674 \\
\hline
\end{tabular}




\begin{tabular}{|c|c|c|c|c|c|}
\hline 35 & 1 & 0 & -1.957836 & -0.475940 & 1. 743541 \\
\hline 36 & 1 & 0 & -2.371117 & -2.182732 & 1. 776811 \\
\hline 37 & 6 & 0 & -3.397585 & -1.065131 & 0.272782 \\
\hline 38 & 6 & 0 & -4.461590 & -1.961187 & 0.168164 \\
\hline 39 & 6 & 0 & -3.483121 & 0.113485 & -0.473474 \\
\hline 40 & 6 & 0 & -5.556191 & -1.689606 & -0.650345 \\
\hline 41 & 1 & 0 & -4.428038 & -2.884782 & 0.739844 \\
\hline 42 & 6 & 0 & -4.549073 & 0.396615 & -1.312988 \\
\hline 43 & 6 & 0 & -5.600089 & -0.513700 & -1.393412 \\
\hline 44 & 1 & 0 & -6.371498 & -2.402389 & -0.713750 \\
\hline 45 & 1 & 0 & -4.545252 & 1. 308263 & -1.902752 \\
\hline 46 & 1 & 0 & -6.443800 & -0.304890 & -2.042369 \\
\hline 47 & 8 & 0 & -2.384203 & 0.982565 & -0.421015 \\
\hline 48 & 6 & 0 & -2.413395 & 2. 179304 & 0.241318 \\
\hline 49 & 6 & 0 & -3.709383 & 2. 622689 & 0.854490 \\
\hline 50 & 1 & 0 & -4.122967 & 1. 833189 & 1. 488426 \\
\hline 51 & 1 & 0 & -3.515263 & 3. 519409 & 1. 440264 \\
\hline 52 & 1 & 0 & -4.450258 & 2. 841060 & 0.081156 \\
\hline 53 & 8 & 0 & -1.369817 & 2. 778765 & 0.300260 \\
\hline 54 & 6 & 0 & 3. 667877 & -2.197631 & -1.899369 \\
\hline 55 & 1 & 0 & 4. 388419 & -2.978479 & -2.161817 \\
\hline 56 & 1 & 0 & 2. 667372 & -2.592025 & -2.107411 \\
\hline 57 & 1 & 0 & 3. 859417 & -1.347969 & -2.565569 \\
\hline 58 & 6 & 0 & 3. 581810 & -3.070699 & 0.439090 \\
\hline 59 & 1 & 0 & 3. 706614 & -2.843995 & 1. 505160 \\
\hline 60 & 1 & 0 & 2. 575258 & -3.468398 & 0.284354 \\
\hline 61 & 1 & 0 & 4. 304822 & -3.849268 & 0.177010 \\
\hline 62 & 6 & 0 & 5. 239049 & -1.308182 & -0.153339 \\
\hline 63 & 1 & 0 & 5. 954250 & -2.132078 & -0.232850 \\
\hline 64 & 1 & 0 & 5. 555114 & -0.545037 & -0.872222 \\
\hline 65 & 1 & 0 & 5. 329946 & -0.891649 & 0.857808 \\
\hline 66 & 1 & 0 & -1.096979 & -1.188592 & -0.758003 \\
\hline 67 & 8 & 0 & 0.105246 & 0.381129 & 0.989295 \\
\hline 68 & 1 & 0 & -0.605498 & 0.668896 & 0.385444 \\
\hline 69 & 1 & 0 & 1. 315630 & 1. 210182 & 0.293710 \\
\hline
\end{tabular}

(22) TS9 


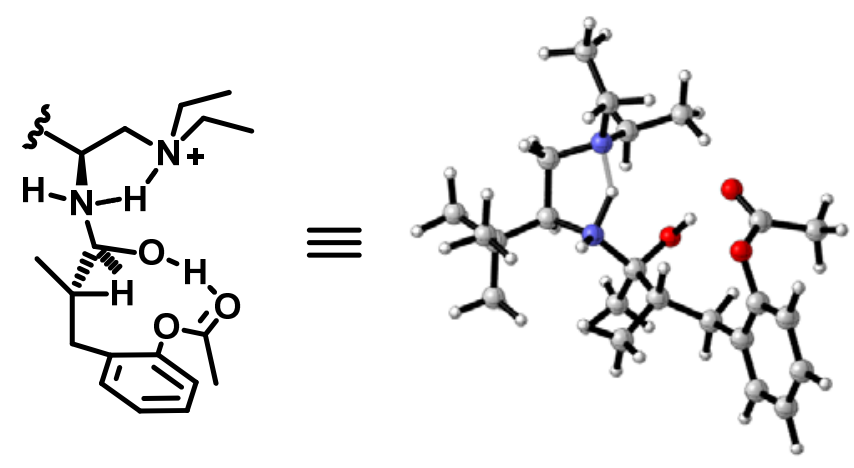

$\mathrm{E}_{\mathrm{sol}}=-1236.012111$

Zero-point correction=

Thermal correction to Energy=

0.626250 (Hartree/Particle)

Thermal correction to Enthalpy=

0.656668

Thermal correction to Gibbs Free Energy=

Sum of electronic and zero-point Energies=

0.657613

Sum of electronic and thermal Energies=

0.567028

$-1234.947285$

$-1234.916867$

Sum of electronic and thermal Enthalpies=

Sum of electronic and thermal Free Energies=

$-1234.915922$

$-1235.006507$

E (Thermal) CV S

KCal/Mol Cal/Mol-Kelvin Cal/Mo1-Kelvin

$\begin{array}{lrrr}\text { Total } & 412.066 & 119.608 & 190.652 \\ \text { Electronic } & 0.000 & 0.000 & 0.000 \\ \text { Translational } & 0.889 & 2.981 & 43.800 \\ \text { Rotational } & 0.889 & 2.981 & 35.984 \\ \text { Vibrational } & 410.288 & 113.646 & 110.867\end{array}$

Standard orientation:

\begin{tabular}{|c|c|c|c|c|c|}
\hline \multirow{2}{*}{$\begin{array}{l}\text { Center } \\
\text { Number }\end{array}$} & \multirow{2}{*}{$\begin{array}{l}\text { Atomic } \\
\text { Number }\end{array}$} & \multirow{2}{*}{$\begin{array}{c}\text { Atomic } \\
\text { Type }\end{array}$} & \multicolumn{3}{|c|}{ Coordinates (Angstroms) } \\
\hline & & & X & Y & Z \\
\hline 1 & 6 & 0 & 3.378626 & -2.206265 & -0.458869 \\
\hline 2 & 6 & 0 & 2.833205 & -0.928852 & 0.227108 \\
\hline 3 & 1 & 0 & 2.961203 & -1.028018 & 1. 309139 \\
\hline 4 & 6 & 0 & 3.571886 & 0.368846 & -0.249625 \\
\hline 5 & 1 & 0 & 3. 868361 & 0.264706 & -1.296511 \\
\hline 6 & 1 & 0 & 4. 476832 & 0.532721 & 0.341549 \\
\hline 7 & 7 & 0 & 1. 391742 & -0.640417 & -0.022792 \\
\hline 8 & 7 & 0 & 2.630373 & 1. 491437 & -0.137378 \\
\hline 9 & 6 & 0 & 2.619073 & 2. 389732 & -1.313606 \\
\hline 10 & 1 & 0 & 2. 436032 & 1. 755408 & -2.188299 \\
\hline 11 & 1 & 0 & 1.749126 & 3. 043867 & -1.211994 \\
\hline 12 & 6 & 0 & 2. 776076 & 2. 178244 & 1. 172594 \\
\hline 13 & 1 & 0 & 2.570775 & 1. 425618 & 1. 939791 \\
\hline
\end{tabular}




\begin{tabular}{|c|c|c|c|c|c|}
\hline 14 & 1 & 0 & 3. 819967 & 2. 498368 & 1. 287620 \\
\hline 15 & 6 & 0 & 1.841429 & 3. 362416 & 1. 355001 \\
\hline 16 & 1 & 0 & 2. 125720 & 4. 208329 & 0.723095 \\
\hline 17 & 1 & 0 & 0.803619 & 3. 105847 & 1. 132303 \\
\hline 18 & 1 & 0 & 1.895835 & 3. 695978 & 2. 394112 \\
\hline 19 & 6 & 0 & 3. 898568 & 3. 194664 & -1.498607 \\
\hline 20 & 1 & 0 & 4. 773801 & 2. 542848 & -1.587636 \\
\hline 21 & 1 & 0 & 3. 832434 & 3. 789850 & -2.412669 \\
\hline 22 & 1 & 0 & 4. 068684 & 3. 883282 & -0.666041 \\
\hline 23 & 1 & 0 & 1. 088098 & -1.077798 & -0.893676 \\
\hline 24 & 6 & 0 & 0.296025 & -0.746484 & 1. 041788 \\
\hline 25 & 6 & 0 & -1.003183 & -1.044353 & 0.253107 \\
\hline 26 & 6 & 0 & 0.595940 & -1.754036 & 2. 136204 \\
\hline 27 & 1 & 0 & -0.263441 & -1.783864 & 2. 810608 \\
\hline 28 & 1 & 0 & 0.774731 & -2.755147 & 1. 748087 \\
\hline 29 & 1 & 0 & 1. 455150 & -1.432369 & 2. 729414 \\
\hline 30 & 6 & 0 & -1.067732 & -2.466389 & -0.308879 \\
\hline 31 & 1 & 0 & -1.111950 & -3.210385 & 0.493474 \\
\hline 32 & 1 & 0 & -1.969628 & -2.579126 & -0.916712 \\
\hline 33 & 1 & 0 & -0.215843 & -2.717674 & -0.951919 \\
\hline 34 & 6 & 0 & -2.258404 & -0.752960 & 1. 101867 \\
\hline 35 & 1 & 0 & -2.112938 & 0.180173 & 1. 659871 \\
\hline 36 & 1 & 0 & -2.375506 & -1.535792 & 1. 859884 \\
\hline 37 & 6 & 0 & -3.533413 & -0.689421 & 0.287190 \\
\hline 38 & 6 & 0 & -4.586962 & -1.578211 & 0.503340 \\
\hline 39 & 6 & 0 & -3.710395 & 0.269278 & -0.717957 \\
\hline 40 & 6 & 0 & -5.757188 & -1.512879 & -0.250519 \\
\hline 41 & 1 & 0 & -4.484081 & -2.335274 & 1. 276154 \\
\hline 42 & 6 & 0 & -4.860108 & 0.351367 & -1.485723 \\
\hline 43 & 6 & 0 & -5.894528 & -0.550391 & -1.245860 \\
\hline 44 & 1 & 0 & -6.558832 & -2.218938 & -0.062030 \\
\hline 45 & 1 & 0 & -4.928517 & 1. 106727 & -2.261802 \\
\hline 46 & 1 & 0 & -6.801254 & -0.500313 & -1.839085 \\
\hline 47 & 8 & 0 & -2.639626 & 1. 119239 & -1.012378 \\
\hline 48 & 6 & 0 & -2.274113 & 2. 068956 & -0.123155 \\
\hline 49 & 6 & 0 & -3.361957 & 2. 714613 & 0.687023 \\
\hline 50 & 1 & 0 & -3.784449 & 1. 996147 & 1. 396318 \\
\hline 51 & 1 & 0 & -2.942660 & 3. 561411 & 1. 227627 \\
\hline 52 & 1 & 0 & -4.175170 & 3. 040331 & 0.032887 \\
\hline 53 & 8 & 0 & -1.097513 & 2. 352931 & -0.054149 \\
\hline 54 & 6 & 0 & 3. 061999 & -2.240062 & -1.961470 \\
\hline 55 & 1 & 0 & 3. 558393 & -3.103998 & -2.413153 \\
\hline 56 & 1 & 0 & 1.990413 & -2.366697 & -2.162974 \\
\hline 57 & 1 & 0 & 3.410801 & -1.352618 & -2.4987 \\
\hline
\end{tabular}




$\begin{array}{rrrrrr}58 & 6 & 0 & 2.789294 & -3.464987 & 0.186409 \\ 59 & 1 & 0 & 2.980887 & -3.491032 & 1.264463 \\ 60 & 1 & 0 & 1.709114 & -3.539128 & 0.021025 \\ 61 & 1 & 0 & 3.245793 & -4.355556 & -0.256347 \\ 62 & 6 & 0 & 4.902165 & -2.227418 & -0.256193 \\ 63 & 1 & 0 & 5.309952 & -3.171787 & -0.627717 \\ 64 & 1 & 0 & 5.406844 & -1.419915 & -0.795509 \\ 65 & 1 & 0 & 5.161784 & -2.148291 & 0.805836 \\ 66 & 1 & 0 & -1.002780 & -0.338226 & -0.588029 \\ 67 & 8 & 0 & 0.254789 & 0.496537 & 1.677700 \\ 68 & 1 & 0 & -0.158190 & 1.154947 & 1.084095 \\ 69 & 1 & 0 & 1.577067 & 0.556158 & -0.161659\end{array}$

(23) Int9

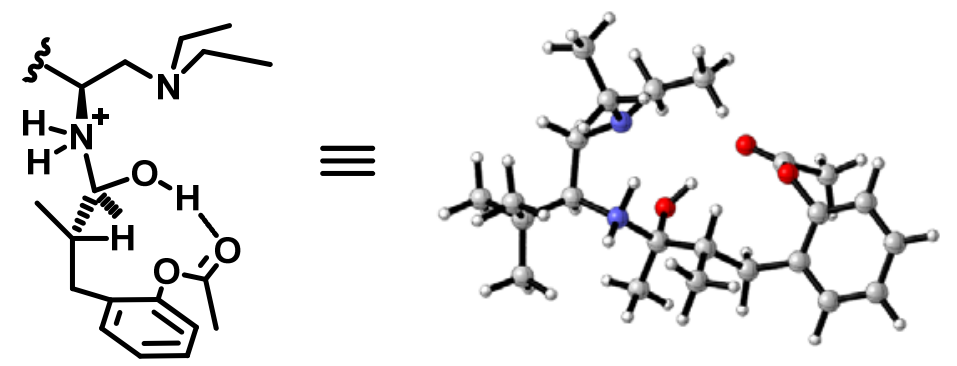

$\mathrm{E}_{\mathrm{sol}}=-1236.021767$

Zero-point correction=

0.629308 (Hartree/Particle)

Thermal correction to Energy=

0. 660020

Thermal correction to Enthalpy=

0.660964

Thermal correction to Gibbs Free Energy=

0.569849

Sum of electronic and zero-point Energies=

$-1234.951765$

Sum of electronic and thermal Energies=

$-1234.921053$

Sum of electronic and thermal Enthalpies=

$-1234.920108$

Sum of electronic and thermal Free Energies=

$-1235.011224$

$\begin{array}{lccc} & \text { E (Thermal) } & \text { CV } & \text { S } \\ & \text { KCal/Mol } & \text { Cal/Mol-Kelvin } & \text { Cal/Mol-Kelvin } \\ \text { Total } & 414.169 & 120.433 & 191.768 \\ \text { Electronic } & 0.000 & 0.000 & 0.000 \\ \text { Translational } & 0.889 & 2.981 & 43.800 \\ \text { Rotational } & 0.889 & 2.981 & 35.905 \\ \text { Vibrational } & 412.391 & 114.471 & 112.063\end{array}$

Standard orientation:

\begin{tabular}{lllll} 
Center & Atomic & Atomic & \multicolumn{2}{c}{ Coordinates (Angstroms) } \\
Number & Number & Type & $X$ & Y
\end{tabular}

1

6

0

$4.115029-1.351159$

0.002061 


\begin{tabular}{|c|c|c|c|c|c|}
\hline 2 & 6 & 0 & 2. 908916 & -0.543183 & 0.540091 \\
\hline 3 & 1 & 0 & 2. 743719 & -0.827227 & 1. 581771 \\
\hline 4 & 6 & 0 & 3. 078671 & 1. 023404 & 0.48616 \\
\hline 5 & 1 & 0 & 4. 070447 & 1. 269013 & 0.0976 \\
\hline 6 & 1 & 0 & 3. 029761 & 1. 406531 & 1. 50751 \\
\hline 7 & 7 & 0 & 1. 635801 & -0.854394 & -0.1879 \\
\hline 8 & 7 & 0 & 2. 033828 & 1. 658503 & -0.3240 \\
\hline 9 & 6 & 0 & 2.508091 & 2. 108220 & -1.6489 \\
\hline 10 & 1 & 0 & 3. 023696 & 1. 260354 & -2.1126 \\
\hline 11 & 1 & 0 & 1.626400 & 2. 305896 & -2.2655 \\
\hline 12 & 6 & 0 & 1. 292262 & 2. 694691 & 0.4244 \\
\hline 13 & 1 & 0 & 0.945559 & 2. 234523 & 1.3537 \\
\hline 14 & 1 & 0 & 1. 969421 & 3. 513438 & 0.7077 \\
\hline 15 & 6 & 0 & 0.100376 & 3. 238181 & -0.3446 \\
\hline 16 & 1 & 0 & 0.401836 & 3. 841145 & -1.2059 \\
\hline 17 & 1 & 0 & -0.546027 & 2. 424760 & -0.6903 \\
\hline 18 & 1 & 0 & -0.493384 & 3. 872779 & $0.3174 t$ \\
\hline 19 & 6 & 0 & 3. 419044 & 3. 332689 & -1.6358 \\
\hline 20 & 1 & 0 & 4. 293576 & 3. 186526 & -0.9935 \\
\hline 21 & 1 & 0 & 3. 779588 & 3. 535838 & -2.6475 \\
\hline 22 & 1 & 0 & 2. 888438 & 4. 223440 & -1.2879 \\
\hline 23 & 1 & 0 & 1. 785837 & -1.521184 & -0.9475 \\
\hline 24 & 6 & 0 & 0.421692 & -1.263156 & 0.66506 \\
\hline 25 & 6 & 0 & -0.802178 & -1.212127 & -0.2728 \\
\hline 26 & 6 & 0 & 0.666591 & -2.621114 & 1. 2974 \\
\hline 27 & 1 & 0 & -0.211893 & -2.892025 & 1.88656 \\
\hline 28 & 1 & 0 & 0.849833 & -3.399937 & 0.5534 \\
\hline 29 & 1 & 0 & 1. 514050 & -2.573717 & 1.98427 \\
\hline 30 & 6 & 0 & -0.683211 & -2.102522 & -1.5126 \\
\hline 31 & 1 & 0 & -0.514286 & -3.151002 & -1.2449 \\
\hline 32 & 1 & 0 & -1.616303 & -2.056239 & -2.0811 \\
\hline 33 & 1 & 0 & 0.110063 & -1.783132 & -2.1972 \\
\hline 34 & 6 & 0 & -2.092758 & -1.552902 & 0.5076 \\
\hline 35 & 1 & 0 & -2.033502 & -1.115714 & 1.5133 \\
\hline 36 & 1 & 0 & -2.156568 & -2.638188 & 0.6455 \\
\hline 37 & 6 & 0 & -3.340818 & -1.081503 & -0.2029 \\
\hline 38 & 6 & 0 & -4.271176 & -1.964552 & -0.7518 \\
\hline 39 & 6 & 0 & -3.595804 & 0.285402 & -0.3477 \\
\hline 40 & 6 & 0 & -5.402186 & -1.495442 & -1.4178 \\
\hline 41 & 1 & 0 & -4.105759 & -3.034396 & -0.6530 \\
\hline 42 & 6 & 0 & -4.706408 & 0.777270 & -1.0128 \\
\hline 43 & 6 & 0 & -5.619909 & -0.127302 & -1.5507 \\
\hline 44 & 1 & 0 & -6.112126 & -2.201465 & -1.8352 \\
\hline 45 & 1 & 0 & -4.839368 & 1. 850121 & -1.1100 \\
\hline
\end{tabular}




\begin{tabular}{|c|c|c|c|c|c|}
\hline 46 & 1 & 0 & -6.496723 & 0.239505 & -2.073626 \\
\hline 47 & 8 & 0 & -2.631328 & 1. 170197 & 0.139727 \\
\hline 48 & 6 & 0 & -2.578573 & 1. 456849 & 1. 467414 \\
\hline 49 & 6 & 0 & -3.861705 & 1. 393050 & 2. 243892 \\
\hline 50 & 1 & 0 & -4.196037 & 0.353466 & 2. 324848 \\
\hline 51 & 1 & 0 & -3.687030 & 1. 800118 & 3. 238155 \\
\hline 52 & 1 & 0 & -4.650729 & 1. 948841 & 1. 731369 \\
\hline 53 & 8 & 0 & -1.503170 & 1. 752698 & 1. 931178 \\
\hline 54 & 6 & 0 & 4. 458436 & -0.996783 & -1.450341 \\
\hline 55 & 1 & 0 & 5.278262 & -1.632830 & -1.797456 \\
\hline 56 & 1 & 0 & 3. 618213 & -1.161812 & -2.138536 \\
\hline 57 & 1 & 0 & 4. 788751 & 0.041065 & -1.557345 \\
\hline 58 & 6 & 0 & 3. 830385 & -2.856995 & 0.094385 \\
\hline 59 & 1 & 0 & 3. 593319 & -3.158728 & 1. 119504 \\
\hline 60 & 1 & 0 & 3. 007140 & -3.179405 & -0.556557 \\
\hline 61 & 1 & 0 & 4. 714823 & -3.418257 & -0.220760 \\
\hline 62 & 6 & 0 & 5. 319099 & -1.042170 & 0.903968 \\
\hline 63 & 1 & 0 & 6. 183683 & -1.629077 & 0.580393 \\
\hline 64 & 1 & 0 & 5. 609174 & 0.012161 & 0.868312 \\
\hline 65 & 1 & 0 & 5. 107503 & -1.303307 & 1. 946492 \\
\hline 66 & 1 & 0 & -0.877303 & -0.166163 & -0.604480 \\
\hline 67 & 8 & 0 & 0.352683 & -0.331295 & 1. 686990 \\
\hline 68 & 1 & 0 & -0.208215 & 0.438791 & 1. 456329 \\
\hline 69 & 1 & 0 & 1. 396078 & 0.130849 & -0.572333 \\
\hline
\end{tabular}

\section{(24) TS10}
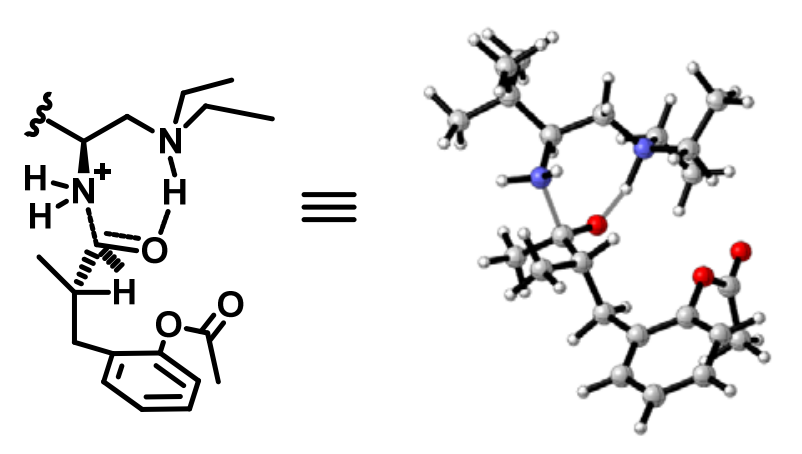

$\mathrm{E}_{\mathrm{sol}}=-1236.015972$

Zero-point correction=

Thermal correction to Energy=

0. 624643 (Hartree/Particle)

Thermal correction to Enthalpy=

0.655889

Thermal correction to Gibbs Free Energy=

Sum of electronic and zero-point Energies=

0.656833

Sum of electronic and thermal Energies=

0.562647

$-1234.943488$

$-1234.912242$

Sum of electronic and thermal Enthalpies=

Sum of electronic and thermal Free Energies=

$-1234.911298$

$-1235.005484$ 
E (Therma1)

KCa1/Mol

Total

Electronic

Translational

Rotational

Vibrational
411. 577

0.000

0.889

0.889

409. 799
$\mathrm{CV}$

Cal/Mo1-Kelvin 120. 363

0.000

2. 981

2. 981

114. 401
S

Cal/Mol-Kelvin

198. 232

0.000

43. 800

35. 924

118. 508

Standard orientation:

\begin{tabular}{|c|c|c|c|c|c|}
\hline \multirow{2}{*}{$\begin{array}{l}\text { Center } \\
\text { Number }\end{array}$} & \multirow{2}{*}{$\begin{array}{l}\text { Atomic } \\
\text { Number }\end{array}$} & \multirow{2}{*}{$\begin{array}{l}\text { Atomic } \\
\text { Type }\end{array}$} & \multicolumn{3}{|c|}{ Coordinates (Angstroms) } \\
\hline & & & $X$ & Y & Z \\
\hline 1 & 6 & 0 & -4.382816 & -1.057761 & 0.337673 \\
\hline 2 & 6 & 0 & -2.990852 & -0.453126 & 0.016613 \\
\hline 3 & 1 & 0 & -2.966904 & -0.195726 & -1.049864 \\
\hline 4 & 6 & 0 & -2.650192 & 0.797665 & 0.839621 \\
\hline 5 & 1 & 0 & -2.335538 & 0.504765 & 1. 848235 \\
\hline 6 & 1 & 0 & -3.534429 & 1. 429354 & 0.953412 \\
\hline 7 & 7 & 0 & -1.884674 & -1.438730 & 0.198197 \\
\hline 8 & 7 & 0 & -1.560461 & 1. 600891 & 0.222753 \\
\hline 9 & 6 & 0 & -0.651692 & 2.218342 & 1. 235390 \\
\hline 10 & 1 & 0 & -0.290496 & 1. 392547 & 1. 858398 \\
\hline 11 & 1 & 0 & 0.214550 & 2.606617 & 0.695399 \\
\hline 12 & 6 & 0 & -2.133797 & 2.575200 & -0.756144 \\
\hline 13 & 1 & 0 & -2.759429 & 1.985317 & -1.433734 \\
\hline 14 & 1 & 0 & -2.788780 & 3. 263983 & -0.211223 \\
\hline 15 & 6 & 0 & -1.063011 & 3. 309864 & -1.541289 \\
\hline 16 & 1 & 0 & -0.472277 & 3. 979601 & -0.913064 \\
\hline 17 & 1 & 0 & -0.380622 & 2.603115 & -2.020026 \\
\hline 18 & 1 & 0 & -1.545422 & 3. 907787 & -2.318311 \\
\hline 19 & 6 & 0 & -1.317965 & 3. 288735 & 2. 086158 \\
\hline 20 & 1 & 0 & -2.216849 & 2. 919748 & 2. 591349 \\
\hline 21 & 1 & 0 & -0.617615 & 3. 618578 & 2. 857018 \\
\hline 22 & 1 & 0 & -1.587876 & 4. 167447 & 1. 494085 \\
\hline 23 & 1 & 0 & -2.236262 & -2.392898 & 0.101137 \\
\hline 24 & 6 & 0 & -0.567096 & -1.229237 & -0.986634 \\
\hline 25 & 6 & 0 & 0.671104 & -1.533727 & -0.126649 \\
\hline 26 & 6 & 0 & -0.931937 & -2.267620 & -2.035855 \\
\hline 27 & 1 & 0 & -0.181584 & -2.219621 & -2.830240 \\
\hline 28 & 1 & 0 & -0.965013 & -3.290004 & -1.649294 \\
\hline 29 & 1 & 0 & -1.894942 & -2.009617 & -2.486329 \\
\hline 30 & 6 & 0 & 0.700583 & -2.909956 & 0.538766 \\
\hline 31 & 1 & 0 & 0.738569 & -3.710112 & -0.207988 \\
\hline
\end{tabular}




\begin{tabular}{|c|c|c|c|c|c|}
\hline 32 & 1 & 0 & 1.595470 & -3.000722 & 1. 160485 \\
\hline 33 & 1 & 0 & -0.158713 & -3.103399 & 1. 191533 \\
\hline 34 & 6 & 0 & 1.924657 & -1.332813 & -1.008550 \\
\hline 35 & 1 & 0 & 1. 767563 & -0.439193 & -1.621777 \\
\hline 36 & 1 & 0 & 2. 030578 & -2.185949 & -1.689411 \\
\hline 37 & 6 & 0 & 3. 175402 & -1.201078 & -0.177657 \\
\hline 38 & 6 & 0 & 4. 080202 & -2.251554 & -0.012698 \\
\hline 39 & 6 & 0 & 3. 449169 & -0.007055 & 0.493826 \\
\hline 40 & 6 & 0 & 5. 206883 & -2.114385 & 0.794529 \\
\hline 41 & 1 & 0 & 3. 896509 & -3.189508 & -0.531079 \\
\hline 42 & 6 & 0 & 4. 559460 & 0.147851 & 1. 311420 \\
\hline 43 & 6 & 0 & 5. 445598 & -0.915565 & 1. 460879 \\
\hline 44 & 1 & 0 & 5. 897324 & -2.944018 & 0.903737 \\
\hline 45 & 1 & 0 & 4. 714934 & 1. 094324 & 1. 820344 \\
\hline 46 & 1 & 0 & 6. 318712 & -0.805444 & 2. 095372 \\
\hline 47 & 8 & 0 & 2.541735 & 1. 045728 & 0.374493 \\
\hline 48 & 6 & 0 & 2. 722044 & 2. 000623 & -0.586906 \\
\hline 49 & 6 & 0 & 3. 928799 & 1. 878963 & -1.478895 \\
\hline 50 & 1 & 0 & 3. 940819 & 0.911821 & -1.989690 \\
\hline 51 & 1 & 0 & 3. 891764 & 2. 688260 & -2.205741 \\
\hline 52 & 1 & 0 & 4. 849068 & 1. 945623 & -0.892359 \\
\hline 53 & 8 & 0 & 1. 907892 & 2. 881968 & -0.661081 \\
\hline 54 & 6 & 0 & -4.528650 & -1.372482 & 1. 831271 \\
\hline 55 & 1 & 0 & -5.496894 & -1.845532 & 2. 019919 \\
\hline 56 & 1 & 0 & -3.756510 & -2.067902 & 2. 183649 \\
\hline 57 & 1 & 0 & -4.484668 & -0.468135 & 2. 447527 \\
\hline 58 & 6 & 0 & -4.586598 & -2.340182 & -0.485344 \\
\hline 59 & 1 & 0 & -4.389152 & -2.170352 & -1.550070 \\
\hline 60 & 1 & 0 & -3.969075 & -3.181558 & -0.146052 \\
\hline 61 & 1 & 0 & -5.622993 & -2.675715 & -0.390218 \\
\hline 62 & 6 & 0 & -5.463673 & -0.055152 & -0.094533 \\
\hline 63 & 1 & 0 & -6.451693 & -0.510130 & 0.018385 \\
\hline 64 & 1 & 0 & -5.462888 & 0.856124 & 0.510005 \\
\hline 65 & 1 & 0 & -5.345532 & 0.226851 & -1.147023 \\
\hline 66 & 1 & 0 & 0.699543 & -0.746062 & 0.642756 \\
\hline 67 & 8 & 0 & -0.657637 & -0.006096 & -1.418309 \\
\hline 68 & 1 & 0 & -0.948123 & 0.859389 & -0.485340 \\
\hline 69 & 1 & 0 & -1.488816 & -1.370891 & 1. 138658 \\
\hline
\end{tabular}

\section{(25) s-Product}



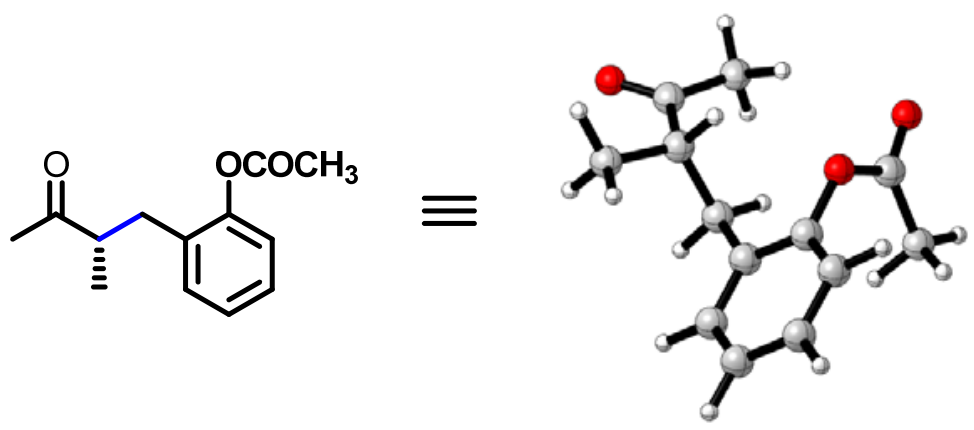

$\mathrm{E}_{\mathrm{sol}}=-730.624107$

Zero-point correction=

0.268410 (Hartree/Particle)

Thermal correction to Energy=

0.284597

Thermal correction to Enthalpy=

0.285542

Thermal correction to Gibbs Free Energy=

0.223607

Sum of electronic and zero-point Energies=

$-730.122064$

Sum of electronic and thermal Energies=

$-730.105877$

Sum of electronic and thermal Enthalpies=

Sum of electronic and thermal Free Energies=

$-730.104932$

$-730.166867$

\section{E (Therma1)}

KCa1/Mo1

Total

Electronic

Translational

Rotational

Vibrational
178. 588

0.000

0.889

0.889

176. 810
$\mathrm{CV}$

Cal/Mo1-Kelvin

59. 827

0. 000

2. 981

2. 981

53. 865
S

Cal/Mo1-Kelvin

130. 352

0.000

42.070

32. 554

55. 729

Standard orientation:

\begin{tabular}{|c|c|c|c|c|c|}
\hline \multirow{2}{*}{$\begin{array}{l}\text { Center } \\
\text { Number }\end{array}$} & \multirow{2}{*}{$\begin{array}{l}\text { Atomic } \\
\text { Number }\end{array}$} & \multirow{2}{*}{$\begin{array}{l}\text { Atomic } \\
\text { Type }\end{array}$} & \multicolumn{3}{|c|}{ Coordinates (Angstroms) } \\
\hline & & & X & Y & Z \\
\hline 1 & 6 & 0 & 3. 018264 & -0.300344 & -0.020728 \\
\hline 2 & 6 & 0 & 1. 635289 & -0.859615 & -0.342344 \\
\hline 3 & 6 & 0 & 3. 119384 & 1. 195071 & 0.216626 \\
\hline 4 & 1 & 0 & 2. 354346 & 1.758367 & -0.326700 \\
\hline 5 & 1 & 0 & 2.991349 & 1. 400961 & 1. 285933 \\
\hline 6 & 1 & 0 & 4. 116933 & 1.533174 & -0.068693 \\
\hline 7 & 6 & 0 & 1. 697542 & -2.345994 & -0.663771 \\
\hline 8 & 1 & 0 & 2.073880 & -2.905492 & 0.198373 \\
\hline 9 & 1 & 0 & 0.707449 & -2.727638 & -0.929432 \\
\hline 10 & 1 & 0 & 2.380693 & -2.533176 & -1.495212 \\
\hline 11 & 6 & 0 & 0.661415 & -0.563056 & 0.823628 \\
\hline 12 & 1 & 0 & 0.792592 & 0.473571 & 1. 158785 \\
\hline 13 & 1 & 0 & 0.917700 & -1.206265 & 1. 673680 \\
\hline
\end{tabular}




\begin{tabular}{rrrrrr}
14 & 6 & 0 & -0.775705 & -0.790498 & 0.430146 \\
15 & 6 & 0 & -1.503226 & -1.909494 & 0.836452 \\
16 & 6 & 0 & -1.418312 & 0.123848 & -0.410168 \\
17 & 6 & 0 & -2.817140 & -2.107682 & 0.417099 \\
18 & 1 & 0 & -1.024914 & -2.636693 & 1.487850 \\
19 & 6 & 0 & -2.722417 & -0.059118 & -0.846762 \\
20 & 6 & 0 & -3.427172 & -1.184307 & -0.426925 \\
21 & 1 & 0 & -3.361488 & -2.987500 & 0.744771 \\
22 & 1 & 0 & -3.162282 & 0.674967 & -1.514780 \\
23 & 1 & 0 & -4.447410 & -1.338332 & -0.763033 \\
24 & 8 & 0 & -0.695163 & 1.218328 & -0.869061 \\
25 & 6 & 0 & -0.608654 & 2.355875 & -0.112321 \\
26 & 6 & 0 & -1.552456 & 2.472206 & 1.059816 \\
27 & 1 & 0 & -1.367841 & 1.678022 & 1.789300 \\
28 & 1 & 0 & -1.395467 & 3.445395 & 1.521673 \\
29 & 1 & 0 & -2.590145 & 2.372673 & 0.730278 \\
30 & 8 & 0 & 0.190746 & 3.190238 & -0.428997 \\
31 & 1 & 0 & 1.274248 & -0.295178 & -1.213910 \\
32 & 8 & 0 & 3.991852 & -1.017093 & 0.042637 \\
\hline
\end{tabular}

\section{(26) Catalyst $1 \mathrm{~b}$}

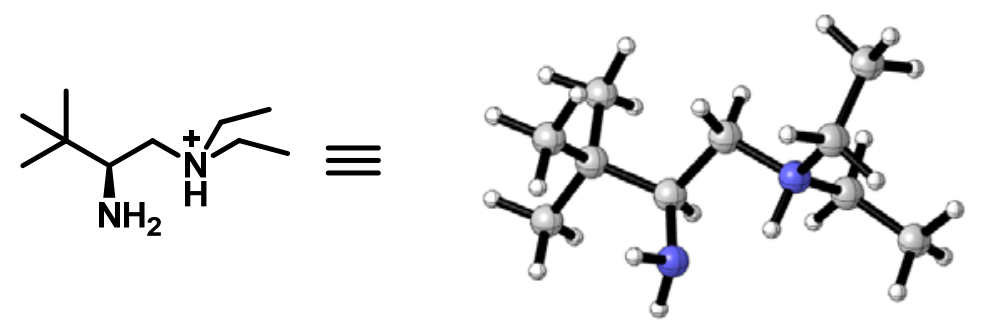

$\mathrm{E}_{\mathrm{sol}}=-505.3966266$

Zero-point correction=

0. 356443 (Hartree/Particle)

Thermal correction to Energy=

0.371868

Thermal correction to Enthalpy=

0.372812

Thermal correction to Gibbs Free Energy=

0.314804

Sum of electronic and zero-point Energies=

$-504.803713$

Sum of electronic and thermal Energies=

$-504.788288$

Sum of electronic and thermal Enthalpies=

$-504.787343$

Sum of electronic and thermal Free Energies=

$-504.845352$

$\begin{array}{lccc} & \text { E (Thermal) } & \text { CV } & \text { S } \\ & \text { KCal/Mol } & \text { Cal/Mol-Kelvin } & \text { Cal/Mo1-Kelvin } \\ \text { Total } & 233.351 & 58.829 & 122.089 \\ \text { Electronic } & 0.000 & 0.000 & 0.000 \\ \text { Translational } & 0.889 & 2.981 & 41.355 \\ \text { Rotational } & 0.889 & 2.981 & 31.350\end{array}$


Vibrational

231.573

52. 867

49. 384

Standard orientation:

\begin{tabular}{|c|c|c|c|c|c|}
\hline \multirow{2}{*}{$\begin{array}{l}\text { Center } \\
\text { Number }\end{array}$} & \multirow{2}{*}{$\begin{array}{l}\text { Atomic } \\
\text { Number }\end{array}$} & \multirow{2}{*}{$\begin{array}{l}\text { Atomic } \\
\text { Type }\end{array}$} & \multicolumn{3}{|c|}{ Coordinates (Angstroms) } \\
\hline & & & $X$ & Y & Z \\
\hline 1 & 6 & 0 & 2. 373349 & 0.109341 & 0.170667 \\
\hline 2 & 6 & 0 & 0.957687 & -0.502239 & 0.001227 \\
\hline 3 & 1 & 0 & 0.847897 & -1.256050 & 0.791462 \\
\hline 4 & 6 & 0 & -0.169066 & 0.538150 & 0.180818 \\
\hline 5 & 1 & 0 & 0.004276 & 1. 408546 & -0.458950 \\
\hline 6 & 1 & 0 & -0.266860 & 0.874914 & 1. 214162 \\
\hline 7 & 7 & 0 & 0.690609 & -1.188945 & -1.269355 \\
\hline 8 & 7 & 0 & -1.467005 & -0.077325 & -0.256853 \\
\hline 9 & 6 & 0 & -2.423329 & 0.912038 & -0.861415 \\
\hline 10 & 1 & 0 & -1.870163 & 1. 411267 & -1.660766 \\
\hline 11 & 1 & 0 & -3.226790 & 0.336030 & -1.324244 \\
\hline 12 & 6 & 0 & -2.069073 & -0.931963 & 0.824301 \\
\hline 13 & 1 & 0 & -1.287987 & -1.636864 & 1. 117894 \\
\hline 14 & 1 & 0 & -2.272223 & -0.277357 & 1. 675135 \\
\hline 15 & 6 & 0 & -3.312867 & -1.671512 & 0.363975 \\
\hline 16 & 1 & 0 & -4.161292 & -1.001260 & 0.207486 \\
\hline 17 & 1 & 0 & -3.127812 & -2.233311 & -0.557053 \\
\hline 18 & 1 & 0 & -3.599432 & -2.388308 & 1. 136719 \\
\hline 19 & 6 & 0 & -2.959855 & 1. 910948 & 0.148127 \\
\hline 20 & 1 & 0 & -2.156407 & 2. 459830 & 0.647568 \\
\hline 21 & 1 & 0 & -3.579507 & 2.640412 & -0.378244 \\
\hline 22 & 1 & 0 & -3.587803 & 1. 434877 & 0.905898 \\
\hline 23 & 1 & 0 & 1. 077292 & -2.128779 & -1.271085 \\
\hline 24 & 6 & 0 & 2. 713222 & 1. 105211 & -0.945277 \\
\hline 25 & 1 & 0 & 3.720056 & 1.500684 & -0.781742 \\
\hline 26 & 1 & 0 & 2. 720654 & 0.638553 & -1.937023 \\
\hline 27 & 1 & 0 & 2. 035980 & 1. 965851 & -0.970319 \\
\hline 28 & 6 & 0 & 3. 390611 & -1.037994 & 0.143950 \\
\hline 29 & 1 & 0 & 3. 193293 & -1.764362 & 0.940611 \\
\hline 30 & 1 & 0 & 3. 383209 & -1.566318 & -0.815421 \\
\hline 31 & 1 & 0 & 4. 401498 & -0.647803 & 0.292556 \\
\hline 32 & 6 & 0 & 2. 454831 & 0.819023 & 1. 528970 \\
\hline 33 & 1 & 0 & 3. 487017 & 1. 117945 & 1. 733219 \\
\hline 34 & 1 & 0 & 1. 845943 & 1. 729456 & 1. 558120 \\
\hline 35 & 1 & 0 & 2. 134912 & 0.159716 & 2. 344852 \\
\hline 36 & 1 & 0 & -1.131039 & -0.727407 & -1.008623 \\
\hline 37 & 1 & 0 & 1. 117002 & -0.692387 & -2.050816 \\
\hline
\end{tabular}




\section{(27) Quione methide}

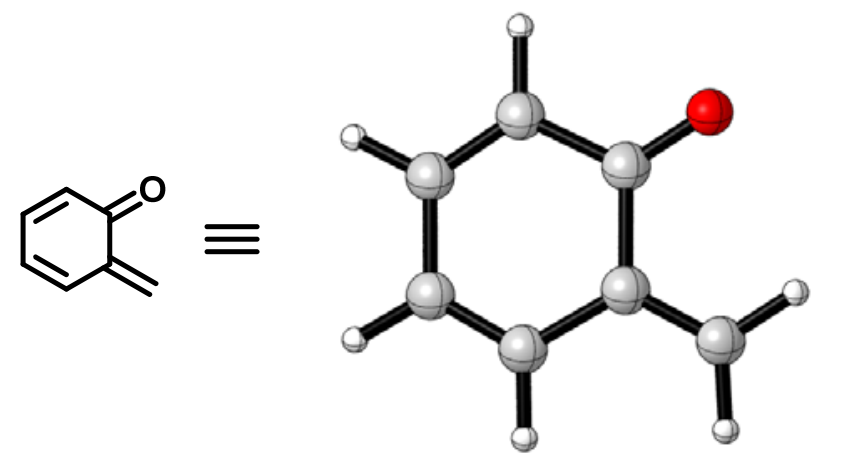

$\mathrm{E}_{\mathrm{sol}}=-345.4900226$

Zero-point correction=

0. 110538 (Hartree/Particle)

Thermal correction to Energy=

0.116938

Thermal correction to Enthalpy=

0.117882

Thermal correction to Gibbs Free Energy=

0.079969

Sum of electronic and zero-point Energies=

$-345.269631$

Sum of electronic and thermal Energies=

$-345.263231$

Sum of electronic and thermal Enthalpies=

$-345.262287$

Sum of electronic and thermal Free Energies=

$-345.300200$

$$
\text { E (Therma1) CV S }
$$

$\begin{array}{lrrr} & \text { KCal/Mol } & \text { Cal/Mol-Kelvin } & \text { Cal/Mol-Kelvin } \\ \text { Total } & 73.379 & 24.673 & 79.794 \\ \text { Electronic } & 0.000 & 0.000 & 0.000 \\ \text { Translational } & 0.889 & 2.981 & 39.893 \\ \text { Rotational } & 0.889 & 2.981 & 27.768 \\ \text { Vibrational } & 71.602 & 18.711 & 12.133\end{array}$

Standard orientation:

\begin{tabular}{rrrrrr} 
Center & Atomic & Atomic & \multicolumn{2}{c}{ Coordinates (Angstroms) } \\
Number & Number & Type & X & Y & $Z$ \\
-1 & 6 & 0 & 1.860910 & -0.578138 & 0.000189 \\
2 & 6 & 0 & 1.749612 & 0.875230 & -0.000144 \\
3 & 6 & 0 & 0.542480 & 1.469747 & -0.000240 \\
4 & 6 & 0 & -0.682968 & 0.679004 & -0.000063 \\
5 & 6 & 0 & -0.582107 & -0.825231 & -0.000102 \\
6 & 6 & 0 & 0.781139 & -1.383299 & 0.000280 \\
7 & 1 & 0 & 2.857565 & -1.012083 & 0.000363 \\
8 & 1 & 0 & 2.659096 & 1.466830 & -0.000282 \\
9 & 1 & 0 & 0.448164 & 2.552718 & -0.000428 \\
10 & 1 & 0 & 0.859951 & -2.465384 & 0.000523 \\
11 & 8 & 0 & -1.573383 & -1.535435 & -0.000373 \\
12 & 6 & 0 & -1.904459 & 1.234409 & 0.000329
\end{tabular}




$\begin{array}{llllll}13 & 1 & 0 & -2.040962 & 2.312078 & 0.000560 \\ 14 & 1 & 0 & -2.784394 & 0.598992 & 0.000750\end{array}$

(28) Int3b

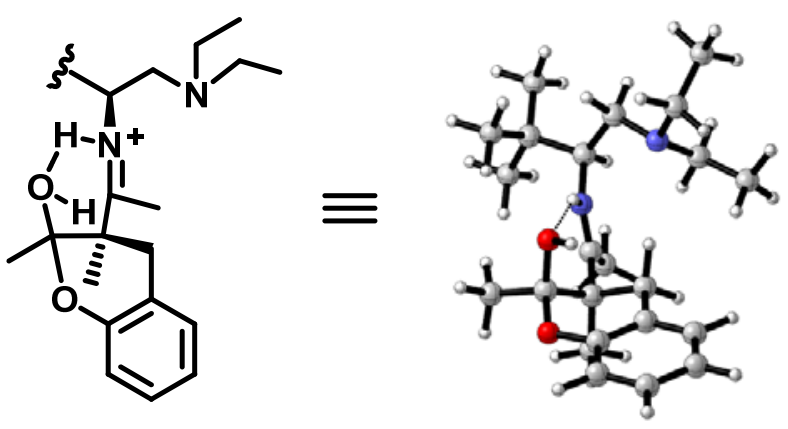

$\mathrm{E}_{\mathrm{sol}}=-1159.578859$

Zero-point correction=

0.601533 (Hartree/Particle)

Thermal correction to Energy=

0.630558

Thermal correction to Enthalpy=

0.631502

Thermal correction to Gibbs Free Energy=

Sum of electronic and zero-point Energies=

0.544134

Sum of electronic and thermal Energies=

Sum of electronic and thermal Enthalpies=

Sum of electronic and thermal Free Energies=

$-1158.566546$

$-1158.537520$

$-1158.536576$

$-1158.623945$

E (Therma1) CV S

$\begin{array}{lrcr} & \text { KCal/Mol } & \text { Cal/Mol-Kelvin } & \text { Cal/Mo1-Kelvin } \\ \text { Total } & 395.681 & 115.029 & 183.883 \\ \text { Electronic } & 0.000 & 0.000 & 0.000 \\ \text { Translational } & 0.889 & 2.981 & 43.660 \\ \text { Rotational } & 0.889 & 2.981 & 35.544 \\ \text { Vibrational } & 393.904 & 109.068 & 104.679\end{array}$

Standard orientation:

\begin{tabular}{|c|c|c|c|c|c|}
\hline \multirow{2}{*}{$\begin{array}{l}\text { Center } \\
\text { Number }\end{array}$} & \multirow{2}{*}{$\begin{array}{l}\text { Atomic } \\
\text { Number }\end{array}$} & \multirow{2}{*}{$\begin{array}{l}\text { Atomic } \\
\text { Type }\end{array}$} & \multicolumn{3}{|c|}{ Coordinates (Angstroms) } \\
\hline & & & $\mathrm{X}$ & Y & Z \\
\hline 1 & 6 & 0 & 3. 461746 & -1.468853 & -0.375962 \\
\hline 2 & 6 & 0 & 4. 930973 & -1.033980 & -0.453680 \\
\hline 3 & 1 & 0 & 5.300320 & -0.689284 & 0.519029 \\
\hline 4 & 1 & 0 & 5.547707 & -1.883184 & -0.762231 \\
\hline 5 & 1 & 0 & 5.082467 & -0.233996 & -1.184319 \\
\hline 6 & 6 & 0 & 3. 354154 & -2.692992 & 0.541103 \\
\hline 7 & 1 & 0 & 3. 747454 & -2.479390 & 1. 542016 \\
\hline 8 & 1 & 0 & 2. 319692 & -3.044749 & 0.639991 \\
\hline 9 & 1 & 0 & 3. 937523 & -3.521211 & 0.128203 \\
\hline 10 & 6 & 0 & 2. 957870 & -1.843180 & -1.776548 \\
\hline
\end{tabular}




\begin{tabular}{|c|c|c|c|c|c|}
\hline 11 & 1 & 0 & 1. 906482 & -2.153771 & -1.772899 \\
\hline 12 & 1 & 0 & 3. 068124 & -1.025991 & -2.495742 \\
\hline 13 & 1 & 0 & 3. 543597 & -2.685479 & -2.156633 \\
\hline 14 & 6 & 0 & 2. 638148 & -0.304822 & 0.240450 \\
\hline 15 & 1 & 0 & 2. 938987 & -0.188072 & 1. 284605 \\
\hline 16 & 6 & 0 & 2. 794019 & 1. 046245 & -0.484457 \\
\hline 17 & 1 & 0 & 2. 804840 & 0.874212 & -1.566971 \\
\hline 18 & 1 & 0 & 3. 779821 & 1. 455463 & -0.210008 \\
\hline 19 & 7 & 0 & 1. 199061 & -0.618179 & 0.215986 \\
\hline 20 & 1 & 0 & -1.375382 & -0.125417 & -1.832869 \\
\hline 21 & 7 & 0 & 1. 696818 & 1. 961153 & -0.194028 \\
\hline 22 & 6 & 0 & 1. 637525 & 3. 055760 & -1.175875 \\
\hline 23 & 1 & 0 & 1. 547427 & 2. 589708 & -2.163580 \\
\hline 24 & 1 & 0 & 0.703779 & 3. 600364 & -1.009321 \\
\hline 25 & 6 & 0 & 1. 738868 & 2. 413094 & 1. 200860 \\
\hline 26 & 1 & 0 & 1. 586271 & 1. 529497 & 1. 838186 \\
\hline 27 & 1 & 0 & 2. 734384 & 2. 812496 & 1. 464015 \\
\hline 28 & 6 & 0 & 0.668801 & 3. 441474 & 1. 545096 \\
\hline 29 & 1 & 0 & 0.868216 & 4. 412524 & 1. 085135 \\
\hline 30 & 1 & 0 & -0.320012 & 3. 106359 & 1. 215420 \\
\hline 31 & 1 & 0 & 0.637944 & 3. 591790 & 2. 627156 \\
\hline 32 & 6 & 0 & 2. 819108 & 4. 025255 & -1.166162 \\
\hline 33 & 1 & 0 & 3.763370 & 3. 519415 & -1.390232 \\
\hline 34 & 1 & 0 & 2. 672364 & 4. 798358 & -1.924841 \\
\hline 35 & 1 & 0 & 2. 924577 & 4. 526598 & -0.198827 \\
\hline 36 & 1 & 0 & 0.734721 & -0.406353 & -0.680967 \\
\hline 37 & 6 & 0 & 0.402142 & -1.018298 & 1. 146041 \\
\hline 38 & 6 & 0 & -1.091341 & -1.056663 & 0.844166 \\
\hline 39 & 6 & 0 & 0.882135 & -1.332657 & 2. 523479 \\
\hline 40 & 1 & 0 & 1. 955523 & -1.211961 & 2. 648037 \\
\hline 41 & 1 & 0 & 0.358898 & -0.692058 & 3. 241548 \\
\hline 42 & 1 & 0 & 0.615424 & -2.365617 & 2. 769403 \\
\hline 43 & 6 & 0 & -1.872116 & -1.846486 & 1. 903077 \\
\hline 44 & 1 & 0 & -1.537878 & -2.884859 & 1. 976329 \\
\hline 45 & 1 & 0 & -1.777316 & -1.385044 & 2. 889061 \\
\hline 46 & 1 & 0 & -2.932400 & -1.854875 & 1. 646035 \\
\hline 47 & 6 & 0 & -1.400423 & -1.642363 & -0.580758 \\
\hline 48 & 8 & 0 & -0.770727 & -0.844379 & -1.580382 \\
\hline 49 & 6 & 0 & -5.504624 & 0.760259 & -0.858111 \\
\hline 50 & 6 & 0 & -4.992815 & 1. 795237 & -0.072791 \\
\hline 51 & 6 & 0 & -3.723243 & 1. 680088 & 0.474800 \\
\hline 52 & 6 & 0 & -2.939304 & 0.544766 & 0.249036 \\
\hline 53 & 6 & 0 & -3.471804 & -0.474083 & -0.541774 \\
\hline 54 & 6 & 0 & -4.746750 & -0.376902 & -1.0953 \\
\hline
\end{tabular}




$\begin{array}{rrrrrr}55 & 1 & 0 & -6.498341 & 0.840936 & -1.285770 \\ 56 & 1 & 0 & -5.585988 & 2.683315 & 0.116038 \\ 57 & 1 & 0 & -3.323417 & 2.477027 & 1.097522 \\ 58 & 1 & 0 & -5.116671 & -1.199614 & -1.697835 \\ 59 & 8 & 0 & -2.783472 & -1.648556 & -0.788842 \\ 60 & 6 & 0 & -1.552158 & 0.424766 & 0.829146 \\ 61 & 1 & 0 & -0.836030 & 1.022130 & 0.247146 \\ 62 & 1 & 0 & -1.536421 & 0.825695 & 1.850396 \\ 63 & 6 & 0 & -0.928426 & -3.068658 & -0.778316 \\ 64 & 1 & 0 & -1.138174 & -3.357730 & -1.809593 \\ 65 & 1 & 0 & 0.147514 & -3.153941 & -0.600670 \\ 66 & 1 & 0 & -1.461872 & -3.742667 & -0.106605\end{array}$

(29) $\mathrm{TS}_{4}$

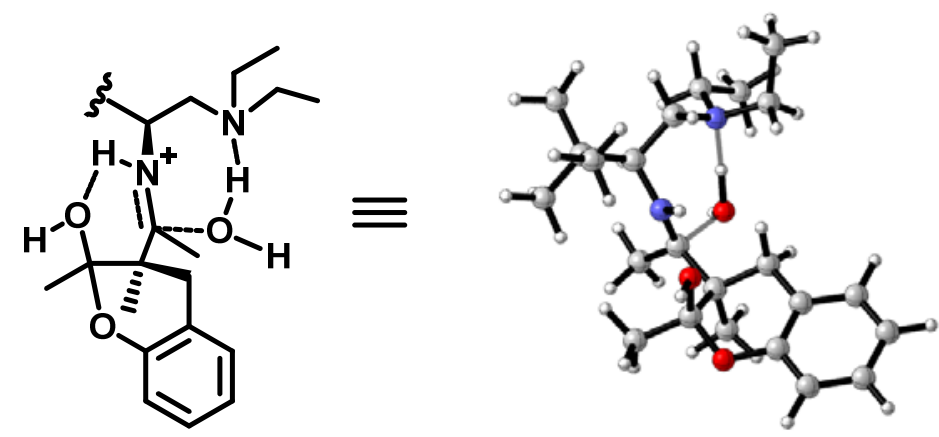

$\mathrm{E}_{\mathrm{sol}}=-1236.005853$

Zero-point correction=

0.626119 (Hartree/Particle)

Thermal correction to Energy=

0.655772

Thermal correction to Enthalpy=

0.656717

Thermal correction to Gibbs Free Energy=

0.568869

Sum of electronic and zero-point Energies=

$-1234.929011$

Sum of electronic and thermal Energies=

$-1234.899357$

Sum of electronic and thermal Enthalpies=

$-1234.898413$

Sum of electronic and thermal Free Energies=

$-1234.986261$

Standard orientation:

\begin{tabular}{|c|c|c|c|c|c|}
\hline \multirow{2}{*}{$\begin{array}{l}\text { Center } \\
\text { Number }\end{array}$} & \multirow{2}{*}{$\begin{array}{l}\text { Atomic } \\
\text { Number }\end{array}$} & \multirow{2}{*}{$\begin{array}{c}\text { Atomic } \\
\text { Type }\end{array}$} & \multicolumn{3}{|c|}{ Coordinates (Angstroms) } \\
\hline & & & $X$ & $\mathrm{Y}$ & Z \\
\hline 1 & 6 & 0 & 2.809379 & -2.132050 & -0.911517 \\
\hline 2 & 6 & 0 & 4. 318904 & -2.076717 & -1.181845 \\
\hline 3 & 1 & 0 & 4. 877532 & -1.786633 & -0.283304 \\
\hline 4 & 1 & 0 & 4. 674945 & -3.065343 & -1.485909 \\
\hline 5 & 1 & 0 & 4.574052 & -1.381718 & -1.987391 \\
\hline 6 & 6 & 0 & 2.523227 & -3.322921 & 0.010195 \\
\hline 7 & 1 & 0 & 3. 096196 & -3.250784 & 0.942658 \\
\hline
\end{tabular}




\begin{tabular}{|c|c|c|c|c|c|}
\hline 8 & 1 & 0 & 1. 459654 & -3.384181 & 0.261085 \\
\hline 9 & 1 & 0 & 2. 805238 & -4.258523 & -0.481735 \\
\hline 10 & 6 & 0 & 2. 047484 & -2.316068 & -2.229674 \\
\hline 11 & 1 & 0 & 0.969310 & -2.417461 & -2.059740 \\
\hline 12 & 1 & 0 & 2.217340 & -1.486125 & -2.925321 \\
\hline 13 & 1 & 0 & 2. 389207 & -3.228197 & -2.7283 \\
\hline 14 & 6 & 0 & 2. 351470 & -0.842019 & -0.183190 \\
\hline 15 & 1 & 0 & 2. 822996 & -0.842690 & 0.806603 \\
\hline 16 & 6 & 0 & 2. 737873 & 0.467765 & -0.892283 \\
\hline 17 & 1 & 0 & 2. 122547 & 0.581576 & -1.791760 \\
\hline 18 & 1 & 0 & 3. 783628 & 0.438529 & -1.215179 \\
\hline 19 & 7 & 0 & 0.898829 & -0.822677 & 0.008329 \\
\hline 20 & 1 & 0 & -1.991685 & -1.983785 & -1.748060 \\
\hline 21 & 7 & 0 & 2. 518162 & 1. 644822 & -0.01898 \\
\hline 22 & 6 & 0 & 2. 011431 & 2. 821281 & -0.770316 \\
\hline 23 & 1 & 0 & 1. 107267 & 2. 487050 & -1.290018 \\
\hline 24 & 1 & 0 & 1. 690727 & 3. 565575 & -0.035432 \\
\hline 25 & 6 & 0 & 3. 739534 & 1. 933496 & 0.7722 \\
\hline 26 & 1 & 0 & 3. 977375 & 1. 018336 & 1. 3277 \\
\hline 27 & 1 & 0 & 4. 584030 & 2. 119587 & 0.096215 \\
\hline 28 & 6 & 0 & 3. 567096 & 3. 097745 & $1.7376 \mathrm{~s}$ \\
\hline 29 & 1 & 0 & 3. 509190 & 4. 056091 & 1. 21552 \\
\hline 30 & 1 & 0 & 2. 663166 & 2. 991043 & 2.3466 \\
\hline 31 & 1 & 0 & 4. 425421 & 3. 141804 & 2. 411738 \\
\hline 32 & 6 & 0 & 3. 003157 & 3. 425949 & -1.755730 \\
\hline 33 & 1 & 0 & 3. 362690 & 2. 683994 & -2.47553 \\
\hline 34 & 1 & 0 & 2.517235 & 4. 226751 & -2.318171 \\
\hline 35 & 1 & 0 & 3. 869287 & 3. 859400 & -1.247447 \\
\hline 36 & 1 & 0 & 0.356598 & -0.670389 & -0.838683 \\
\hline 37 & 6 & 0 & 0.230369 & -0.622938 & 1. 15638 \\
\hline 38 & 6 & 0 & -1.291196 & -0.387399 & 0.98275 \\
\hline 39 & 6 & 0 & 0.707454 & -1.377581 & 2.377216 \\
\hline 40 & 1 & 0 & 1. 796672 & -1.397423 & 2. 442910 \\
\hline 41 & 1 & 0 & 0.303145 & -0.955667 & 3.2970 \\
\hline 42 & 1 & 0 & 0.362613 & -2.413247 & 2. 302827 \\
\hline 43 & 6 & 0 & -1.975765 & -0.287129 & 2. 351335 \\
\hline 44 & 1 & 0 & -1.943711 & -1.224427 & 2. 911810 \\
\hline 45 & 1 & 0 & -1.493414 & 0.494960 & 2. 9451 \\
\hline 46 & 1 & 0 & -3.023286 & -0.009610 & 2. 224763 \\
\hline 47 & 6 & 0 & -1.954009 & -1.536186 & 0.143366 \\
\hline 48 & 8 & 0 & -1.467951 & -1.396328 & -1.179460 \\
\hline 49 & 6 & 0 & -5.695881 & 1. 163996 & -1.014308 \\
\hline 50 & 6 & 0 & -4.901839 & 2. 311232 & -1.011359 \\
\hline 51 & 6 & 0 & -3.572506 & 2. 225729 & -0.618800 \\
\hline
\end{tabular}




$\begin{array}{rrrrrr}52 & 6 & 0 & -3.006113 & 1.008198 & -0.235584 \\ 53 & 6 & 0 & -3.819002 & -0.123623 & -0.256067 \\ 54 & 6 & 0 & -5.156193 & -0.057610 & -0.635616 \\ 55 & 1 & 0 & -6.737780 & 1.220872 & -1.311731 \\ 56 & 1 & 0 & -5.321042 & 3.267146 & -1.306344 \\ 57 & 1 & 0 & -2.953436 & 3.119645 & -0.600620 \\ 58 & 1 & 0 & -5.748125 & -0.966644 & -0.625187 \\ 59 & 8 & 0 & -3.349058 & -1.360859 & 0.138699 \\ 60 & 6 & 0 & -1.564962 & 0.920152 & 0.202314 \\ 61 & 1 & 0 & -0.913917 & 0.988519 & -0.679121 \\ 62 & 1 & 0 & -1.322499 & 1.768910 & 0.845937 \\ 63 & 6 & 0 & -1.733636 & -2.953733 & 0.643051 \\ 64 & 1 & 0 & -2.359599 & -3.627172 & 0.050769 \\ 65 & 1 & 0 & -0.689603 & -3.243339 & 0.507244 \\ 66 & 1 & 0 & -2.023779 & -3.067304 & 1.689660 \\ 67 & 8 & 0 & 0.739941 & 1.061930 & 1.697373 \\ 68 & 1 & 0 & 1.520662 & 1.343224 & 0.958681 \\ 69 & 1 & 0 & 1.123089 & 1.057286 & 2.590003\end{array}$

(30) Int6

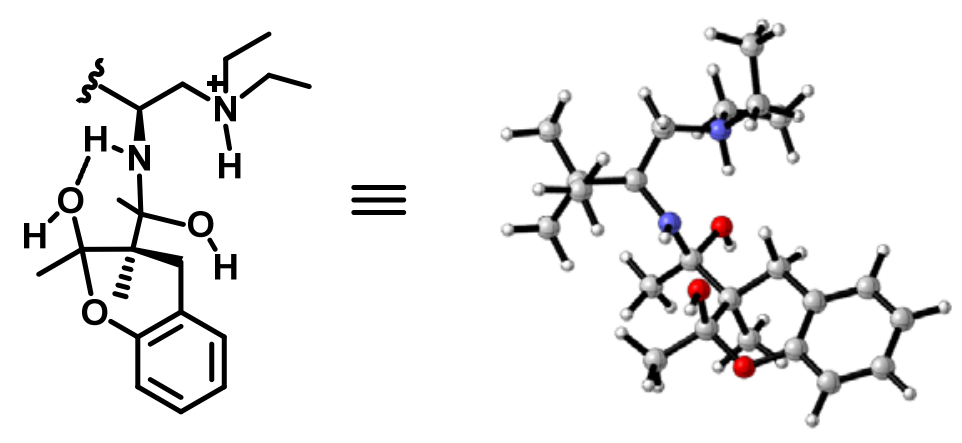

$\mathrm{E}_{\mathrm{sol}}=-1236.028894$

Zero-point correction=

0.631622 (Hartree/Particle)

Thermal correction to Energy=

0.661560

Thermal correction to Enthalpy=

0.662504

Thermal correction to Gibbs Free Energy=

0.573451

Sum of electronic and zero-point Energies=

$-1234.947116$

Sum of electronic and thermal Energies=

Sum of electronic and thermal Enthalpies=

$-1234.917178$

$-1234.916234$

Sum of electronic and thermal Free Energies=

$-1235.005287$

$\begin{array}{lccc} & \text { E (Therma1) } & \text { CV } & \text { S } \\ & \text { KCal/Mol } & \text { Cal/Mol-Kelvin } & \text { Cal/Mol-Kelvin } \\ \text { Total } & 415.135 & 120.989 & 187.427 \\ \text { Electronic } & 0.000 & 0.000 & 0.000 \\ \text { Translational } & 0.889 & 2.981 & 43.800\end{array}$


Rotational

0. 889

2. 981

35. 757

Vibrational

413. 358

115. 028

107. 870

Standard orientation:

\begin{tabular}{|c|c|c|c|c|c|}
\hline \multirow{2}{*}{$\begin{array}{l}\text { Center } \\
\text { Number }\end{array}$} & \multirow{2}{*}{$\begin{array}{l}\text { Atomic } \\
\text { Number }\end{array}$} & \multirow{2}{*}{$\begin{array}{c}\text { Atomic } \\
\text { Type }\end{array}$} & \multicolumn{3}{|c|}{ Coordinates (Angstroms) } \\
\hline & & & $X$ & Y & Z \\
\hline 1 & 6 & 0 & 3. 147545 & -1.817261 & -0.809470 \\
\hline 2 & 6 & 0 & 4. 651132 & -1.569591 & -1.001485 \\
\hline 3 & 1 & 0 & 5.138773 & -1.310401 & -0.053794 \\
\hline 4 & 1 & 0 & 5.130650 & -2.476348 & -1.381516 \\
\hline 5 & 1 & 0 & 4. 853358 & -0.773232 & -1.725787 \\
\hline 6 & 6 & 0 & 2.968179 & -3.099945 & 0.007735 \\
\hline 7 & 1 & 0 & 3. 466440 & -3.028101 & 0.981338 \\
\hline 8 & 1 & 0 & 1.910297 & -3.320321 & 0.177659 \\
\hline 9 & 1 & 0 & 3. 403788 & -3.949024 & -0.527897 \\
\hline 10 & 6 & 0 & 2. 487371 & -2.018753 & -2.181207 \\
\hline 11 & 1 & 0 & 1.433597 & -2.306856 & -2.092715 \\
\hline 12 & 1 & 0 & 2. 548438 & -1.131387 & -2.821021 \\
\hline 13 & 1 & 0 & 2. 995958 & -2.829650 & -2.711520 \\
\hline 14 & 6 & 0 & 2. 509815 & -0.625962 & -0.034366 \\
\hline 15 & 1 & 0 & 2. 900027 & -0.650014 & 0.991768 \\
\hline 16 & 6 & 0 & 2. 935495 & 0.724713 & -0.665884 \\
\hline 17 & 1 & 0 & 2. 702782 & 0.721018 & -1.733870 \\
\hline 18 & 1 & 0 & 3. 998991 & 0.935466 & -0.540572 \\
\hline 19 & 7 & 0 & 1. 061368 & -0.653056 & 0.022958 \\
\hline 20 & 1 & 0 & -1.670572 & -1.798623 & -2.048985 \\
\hline 21 & 7 & 0 & 2. 163250 & 1. 853923 & -0.049076 \\
\hline 22 & 6 & 0 & 1. 727765 & 2. 884953 & -1.051436 \\
\hline 23 & 1 & 0 & 1. 190153 & 2. 331329 & -1.825702 \\
\hline 24 & 1 & 0 & 1. 007354 & 3. 533534 & -0.548279 \\
\hline 25 & 6 & 0 & 2. 867826 & 2. 417133 & 1. 151012 \\
\hline 26 & 1 & 0 & 3. 062015 & 1.560635 & 1. 799371 \\
\hline 27 & 1 & 0 & 3. 822711 & 2. 820137 & 0.805711 \\
\hline 28 & 6 & 0 & 2. 033492 & 3. 459319 & 1. 873780 \\
\hline 29 & 1 & 0 & 1. 953916 & 4. 393055 & 1. 311414 \\
\hline 30 & 1 & 0 & 1. 029449 & 3. 076585 & 2. 080007 \\
\hline 31 & 1 & 0 & 2. 509642 & 3. 691977 & 2. 829006 \\
\hline 32 & 6 & 0 & 2. 884876 & 3. 676994 & -1.632366 \\
\hline 33 & 1 & 0 & 3. 640356 & 3. 025936 & -2.082032 \\
\hline 34 & 1 & 0 & 2. 503013 & 4. 332444 & -2.418280 \\
\hline 35 & 1 & 0 & 3. 367130 & 4. 310615 & -0.883197 \\
\hline 36 & 1 & 0 & 0.582857 & -1.039813 & -0.782495 \\
\hline 37 & 6 & 0 & 0.318231 & -0.726802 & 1. 259240 \\
\hline
\end{tabular}




\begin{tabular}{|c|c|c|c|c|c|}
\hline 38 & 6 & 0 & -1.224429 & -0.632519 & 0.944938 \\
\hline 39 & 6 & 0 & 0.700743 & -1.885503 & 2. 189521 \\
\hline 40 & 1 & 0 & 1. 783289 & -1.893170 & 2. 337751 \\
\hline 41 & 1 & 0 & 0.233321 & -1.770573 & 3. 171750 \\
\hline 42 & 1 & 0 & 0.402572 & -2.855524 & 1. 794926 \\
\hline 43 & 6 & 0 & -2.020625 & -0.819530 & 2. 246423 \\
\hline 44 & 1 & 0 & -1.979980 & -1.842858 & 2. 626350 \\
\hline 45 & 1 & 0 & -1.637980 & -0.152284 & 3. 026913 \\
\hline 46 & 1 & 0 & -3.070154 & -0.561816 & 2. 095126 \\
\hline 47 & 6 & 0 & -1.758545 & -1.651835 & -0.116541 \\
\hline 48 & 8 & 0 & -1.210714 & -1.276873 & -1.371175 \\
\hline 49 & 6 & 0 & -5.599008 & 1. 006079 & -1.064966 \\
\hline 50 & 6 & 0 & -4.886512 & 2. 177039 & -0.806601 \\
\hline 51 & 6 & 0 & -3.580373 & 2. 096319 & -0.340342 \\
\hline 52 & 6 & 0 & -2.956986 & 0.864308 & -0.132860 \\
\hline 53 & 6 & 0 & -3.688694 & -0.290348 & -0.406045 \\
\hline 54 & 6 & 0 & -5.001700 & -0.231182 & -0.864565 \\
\hline 55 & 1 & 0 & -6.622369 & 1. 056391 & -1.422026 \\
\hline 56 & 1 & 0 & -5.351115 & 3. 145335 & -0.958733 \\
\hline 57 & 1 & 0 & -3.025725 & 3. 006182 & -0.121251 \\
\hline 58 & 1 & 0 & -5.530442 & -1.159431 & -1.053834 \\
\hline 59 & 8 & 0 & -3.164428 & -1.548421 & -0.198370 \\
\hline 60 & 6 & 0 & -1.540175 & 0.768186 & 0.375496 \\
\hline 61 & 1 & 0 & -0.844746 & 0.990844 & -0.443656 \\
\hline 62 & 1 & 0 & -1.380841 & 1. 516495 & 1. 158626 \\
\hline 63 & 6 & 0 & -1.501175 & -3.130835 & 0.128864 \\
\hline 64 & 1 & 0 & -2.100596 & -3.699827 & -0.587909 \\
\hline 65 & 1 & 0 & -0.447834 & -3.365906 & -0.038112 \\
\hline 66 & 1 & 0 & -1.797243 & -3.444425 & 1. 131569 \\
\hline 67 & 8 & 0 & 0.715050 & 0.480248 & 1. 952478 \\
\hline 68 & 1 & 0 & 1. 299914 & 1. 400195 & 0.307378 \\
\hline 69 & 1 & 0 & 0.451738 & 0.415851 & 2. 882628 \\
\hline
\end{tabular}

(31) TS11

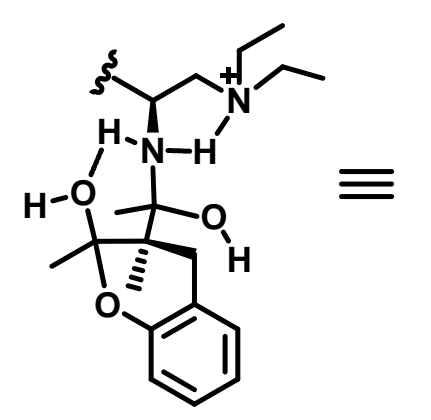

$\mathrm{E}_{\text {sol }}=-1236.012858$

Zero-point correction=

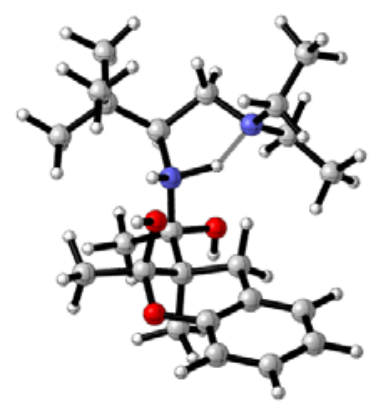

0. 626877 (Hartree/Particle) 
Thermal correction to Energy=

0.656466

Thermal correction to Enthalpy=

0.657411

Thermal correction to Gibbs Free Energy=

0.569963

Sum of electronic and zero-point Energies=

$-1234.939735$

Sum of electronic and thermal Energies=

$-1234.910146$

Sum of electronic and thermal Enthalpies=

$-1234.909202$

Sum of electronic and thermal Free Energies=

$-1234.996649$

\section{E (Therma1)}

KCa1/Mol

Total

411.939

Electronic

Translational

Rotational

Vibrational

Standard orientation:
0. 000
0. 889
0. 889

410. 161
CV

Cal/Mo1-Kelvin 119. 547

0.000

2. 981

2. 981

113. 585
$\mathrm{S}$

Cal/Mol-Kelvin

184. 048

0.000

43. 800

35. 654

104. 594

\begin{tabular}{lllll} 
Center & Atomic & Atomic & \multicolumn{2}{c}{ Coordinates (Angstroms) } \\
Number & Number & Type & X & Y
\end{tabular}

$\begin{array}{rrrrrr}1 & 6 & 0 & 3.404521 & -1.425641 & -0.709390 \\ 2 & 6 & 0 & 4.906981 & -1.098489 & -0.718855 \\ 3 & 1 & 0 & 5.308948 & -1.057975 & 0.300121 \\ 4 & 1 & 0 & 5.449937 & -1.879481 & -1.258632 \\ 5 & 1 & 0 & 5.128576 & -0.147891 & -1.213311 \\ 6 & 6 & 0 & 3.233449 & -2.841684 & -0.152494 \\ 7 & 1 & 0 & 3.611436 & -2.923804 & 0.872114 \\ 8 & 1 & 0 & 2.186708 & -3.159296 & -0.164672 \\ 9 & 1 & 0 & 3.793455 & -3.550173 & -0.770286 \\ 10 & 6 & 0 & 2.860358 & -1.404462 & -2.145589 \\ 11 & 1 & 0 & 1.812929 & -1.727778 & -2.199766 \\ 12 & 1 & 0 & 2.940124 & -0.422060 & -2.622837 \\ 13 & 1 & 0 & 3.435694 & -2.104777 & -2.758493 \\ 14 & 6 & 0 & 2.697948 & -0.379711 & 0.189097 \\ 15 & 1 & 0 & 3.007374 & -0.520060 & 1.229777 \\ 16 & 6 & 0 & 3.056773 & 1.092279 & -0.238165 \\ 17 & 1 & 0 & 3.306735 & 1.120447 & -1.300381 \\ 18 & 1 & 0 & 3.929236 & 1.445352 & 0.317258 \\ 19 & 7 & 0 & 1.209767 & -0.422136 & 0.159755 \\ 20 & 1 & 0 & -1.228788 & -1.386810 & -2.281418 \\ 21 & 7 & 0 & 1.882225 & 1.943465 & -0.001852 \\ 22 & 6 & 0 & 1.452089 & 2.710724 & -1.193394 \\ 23 & 1 & 0 & 1.342567 & 1.984679 & -2.007692 \\ 24 & 1 & 0 & 0.451906 & 3.102188 & -0.987517\end{array}$




\begin{tabular}{|c|c|c|c|c|c|}
\hline 25 & 6 & 0 & 2. 018514 & 2. 741751 & 1. 239616 \\
\hline 26 & 1 & 0 & 2. 251450 & 2. 028592 & 2. 034396 \\
\hline 27 & 1 & 0 & 2. 874028 & 3. 422167 & 1. 135816 \\
\hline 28 & 6 & 0 & 0.758581 & 3. 511551 & 1. 594533 \\
\hline 29 & 1 & 0 & 0.551686 & 4. 318559 & 0.886113 \\
\hline 30 & 1 & 0 & -0.103853 & 2. 841063 & 1. 632467 \\
\hline 31 & 1 & 0 & 0.881033 & 3. 966002 & 2. 580650 \\
\hline 32 & 6 & 0 & 2. 398975 & 3. 834123 & -1.592832 \\
\hline 33 & 1 & 0 & 3. 414675 & 3. 462779 & -1.764022 \\
\hline 34 & 1 & 0 & 2. 051881 & 4. 298449 & -2.519014 \\
\hline 35 & 1 & 0 & 2. 443944 & 4. 613604 & -0.827098 \\
\hline 36 & 1 & 0 & 0.833152 & -0.812563 & -0.710709 \\
\hline 37 & 6 & 0 & 0.391565 & -0.848599 & 1. 336181 \\
\hline 38 & 6 & 0 & -1.128442 & -0.862541 & 0.914279 \\
\hline 39 & 6 & 0 & 0.872912 & -2.133071 & 2. 005071 \\
\hline 40 & 1 & 0 & 1.937890 & -2.061598 & 2. 233317 \\
\hline 41 & 1 & 0 & 0.342269 & -2.263793 & 2. 952160 \\
\hline 42 & 1 & 0 & 0.698595 & -3.027239 & 1. 410728 \\
\hline 43 & 6 & 0 & -1.958537 & -1.412396 & 2. 085904 \\
\hline 44 & 1 & 0 & -1.808679 & -2.481742 & 2. 250826 \\
\hline 45 & 1 & 0 & -1.703081 & -0.883189 & 3. 010332 \\
\hline 46 & 1 & 0 & -3.022118 & -1.247196 & 1. 905224 \\
\hline 47 & 6 & 0 & -1.469082 & -1.687020 & -0.372407 \\
\hline 48 & 8 & 0 & -0.870098 & -0.998936 & -1.465988 \\
\hline 49 & 6 & 0 & -5.540568 & 0.684287 & -1.093089 \\
\hline 50 & 6 & 0 & -5.000700 & 1. 841235 & -0.530380 \\
\hline 51 & 6 & 0 & -3.729672 & 1. 805924 & 0.028322 \\
\hline 52 & 6 & 0 & -2.972492 & 0.632089 & 0.031046 \\
\hline 53 & 6 & 0 & -3.530771 & -0.504585 & -0.546622 \\
\hline 54 & 6 & 0 & -4.806422 & -0.494002 & -1.102572 \\
\hline 55 & 1 & 0 & -6.536049 & 0.700098 & -1.524430 \\
\hline 56 & 1 & 0 & -5.573396 & 2. 762251 & -0.520982 \\
\hline 57 & 1 & 0 & -3.308913 & 2. 701328 & 0.480624 \\
\hline 58 & 1 & 0 & -5.199194 & -1.410904 & -1.529122 \\
\hline 59 & 8 & 0 & -2.862600 & -1.713260 & -0.561701 \\
\hline 60 & 6 & 0 & -1.592231 & 0.584569 & 0.635974 \\
\hline 61 & 1 & 0 & -0.893923 & 1. 094293 & -0.040313 \\
\hline 62 & 1 & 0 & -1.586050 & 1. 139112 & 1. 578546 \\
\hline 63 & 6 & 0 & -1.059174 & -3.152476 & -0.413947 \\
\hline 64 & 1 & 0 & -1.545724 & -3.610842 & -1.279633 \\
\hline 65 & 1 & 0 & 0.021428 & -3.248034 & -0.540100 \\
\hline 66 & 1 & 0 & -1.379018 & -3.699800 & 0.474510 \\
\hline 67 & 8 & 0 & 0.624690 & 0.223253 & 2. 236760 \\
\hline 68 & 1 & 0 & 1. 100881 & 0.788678 & 0.1187 \\
\hline
\end{tabular}


(32) Int1o
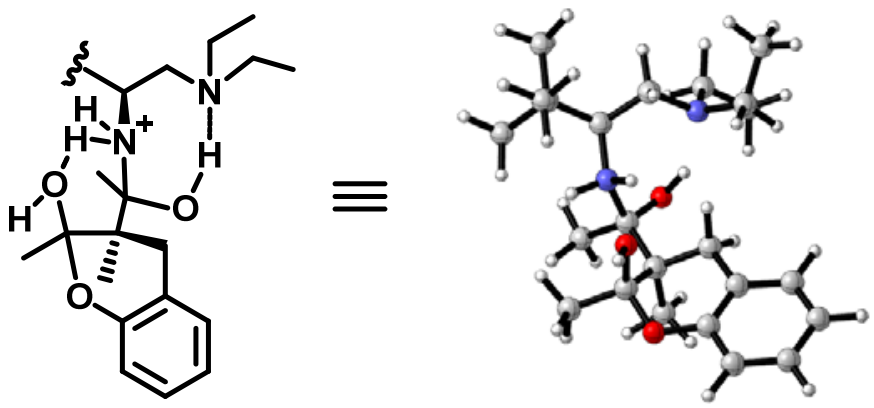

$\mathrm{E}_{\mathrm{sol}}=-1236.02461$

Zero-point correction=

0.631463 (Hartree/Particle)

Thermal correction to Energy=

0.661102

Thermal correction to Enthalpy=

0.662047

Thermal correction to Gibbs Free Energy=

Sum of electronic and zero-point Energies=

0.574248

Sum of electronic and thermal Energies=

$-1234.943157$

$-1234.913518$

Sum of electronic and thermal Enthalpies=

$-1234.912574$

Sum of electronic and thermal Free Energies=

$-1235.000372$

E (Therma1)

KCal/Mol

Total

Electronic

Translational

Rotational

Vibrational
414. 848

0.000

0.889

0.889

413. 071
$\mathrm{CV}$

Cal/Mo1-Kelvin 119. 644

0.000

2. 981

2. 981

113. 683
S

Cal/Mo1-Kelvin

184. 787

0.000

43. 800

35.725

105. 263

Standard orientation:

\begin{tabular}{|c|c|c|c|c|c|}
\hline \multirow{2}{*}{$\begin{array}{l}\text { Center } \\
\text { Number }\end{array}$} & \multirow{2}{*}{$\begin{array}{l}\text { Atomic } \\
\text { Number }\end{array}$} & \multirow{2}{*}{$\begin{array}{c}\text { Atomic } \\
\text { Type }\end{array}$} & \multicolumn{3}{|c|}{ Coordinates (Angstroms) } \\
\hline & & & $\mathrm{X}$ & Y & Z \\
\hline 1 & 6 & 0 & 3. 405500 & -1.519980 & -0.906578 \\
\hline 2 & 6 & 0 & 4. 854620 & -1.012144 & -0.964570 \\
\hline 3 & 1 & 0 & 5. 210367 & -0.702149 & 0.024609 \\
\hline 4 & 1 & 0 & 5.509244 & -1.814427 & -1.316516 \\
\hline 5 & 1 & 0 & 4. 972513 & -0.172874 & -1.654840 \\
\hline 6 & 6 & 0 & 3. 411486 & -2.851987 & -0.138916 \\
\hline 7 & 1 & 0 & 3. 811285 & -2.724813 & 0.873056 \\
\hline 8 & 1 & 0 & 2. 423028 & -3.323915 & -0.054763 \\
\hline 9 & 1 & 0 & 4. 048173 & -3.571556 & -0.661083 \\
\hline 10 & 6 & 0 & 2. 883496 & -1.746293 & -2.333039 \\
\hline 11 & 1 & 0 & 1. 845824 & -2.105521 & -2.355273 \\
\hline
\end{tabular}




\begin{tabular}{|c|c|c|c|c|c|}
\hline 12 & 1 & 0 & 2. 939532 & -0.836179 & -2.938221 \\
\hline 13 & 1 & 0 & 3. 492687 & -2.506223 & -2.830932 \\
\hline 14 & 6 & 0 & 2. 540196 & -0.484247 & -0.141337 \\
\hline 15 & 1 & 0 & 2. 892047 & -0.426289 & 0.893542 \\
\hline 16 & 6 & 0 & 2. 518147 & 0.918687 & -0.768426 \\
\hline 17 & 1 & 0 & 2. 010082 & 0.859952 & -1.739900 \\
\hline 18 & 1 & 0 & 3. 550510 & 1. 231833 & -0.970062 \\
\hline 19 & 7 & 0 & 1. 109333 & -0.973795 & -0.068413 \\
\hline 20 & 1 & 0 & -1.667311 & -1.807653 & -2.043465 \\
\hline 21 & 7 & 0 & 1. 822602 & 1. 906921 & 0.065011 \\
\hline 22 & 6 & 0 & 1. 158601 & 2. 953289 & -0.740114 \\
\hline 23 & 1 & 0 & 0.500380 & 2. 440197 & -1.450807 \\
\hline 24 & 1 & 0 & 0.504633 & 3. 519438 & -0.069911 \\
\hline 25 & 6 & 0 & 2. 748443 & 2. 453505 & 1. 082613 \\
\hline 26 & 1 & 0 & 3. 142325 & 1.601200 & 1. 647030 \\
\hline 27 & 1 & 0 & 3. 606483 & 2. 939571 & 0.595030 \\
\hline 28 & 6 & 0 & 2. 068923 & 3. 412352 & 2. 048849 \\
\hline 29 & 1 & 0 & 1. 776425 & 4. 347275 & 1. 563370 \\
\hline 30 & 1 & 0 & 1. 179915 & 2. 956163 & 2. 497195 \\
\hline 31 & 1 & 0 & 2. 759182 & 3. 663681 & 2. 857379 \\
\hline 32 & 6 & 0 & 2. 095876 & 3. 896935 & -1.488445 \\
\hline 33 & 1 & 0 & 2. 776900 & 3. 351338 & -2.150249 \\
\hline 34 & 1 & 0 & 1. 511570 & 4. 581963 & -2.107850 \\
\hline 35 & 1 & 0 & 2. 696120 & 4. 501317 & -0.802661 \\
\hline 36 & 1 & 0 & 0.542577 & -0.514694 & -0.793477 \\
\hline 37 & 6 & 0 & 0.345684 & -0.848960 & 1. 292522 \\
\hline 38 & 6 & 0 & -1.188657 & -0.759625 & 0.972429 \\
\hline 39 & 6 & 0 & 0.783291 & -2.014153 & 2. 168459 \\
\hline 40 & 1 & 0 & 1. 874107 & -2.019645 & 2. 244244 \\
\hline 41 & 1 & 0 & 0.389927 & -1.847636 & 3. 172154 \\
\hline 42 & 1 & 0 & 0.438318 & -2.985533 & 1. 813586 \\
\hline 43 & 6 & 0 & -1.948880 & -0.947423 & 2. 293779 \\
\hline 44 & 1 & 0 & -1.910610 & -1.976053 & 2. 659882 \\
\hline 45 & 1 & 0 & -1.515327 & -0.292364 & 3. 054747 \\
\hline 46 & 1 & 0 & -2.998879 & -0.676066 & 2. 166176 \\
\hline 47 & 6 & 0 & -1.731159 & -1.766907 & -0.092888 \\
\hline 48 & 8 & 0 & -1.138109 & -1.400919 & -1.337756 \\
\hline 49 & 6 & 0 & -5.523793 & 0.947812 & -1.070311 \\
\hline 50 & 6 & 0 & -4.802146 & 2. 109063 & -0.791468 \\
\hline 51 & 6 & 0 & -3.504679 & 2. 011884 & -0.306017 \\
\hline 52 & 6 & 0 & -2.900949 & 0.769568 & -0.098705 \\
\hline 53 & 6 & 0 & -3.638936 & -0.372453 & -0.395245 \\
\hline 54 & 6 & 0 & -4.943855 & -0.297973 & -0.873310 \\
\hline 55 & 1 & 0 & -6.541003 & 1. 013101 & -1.4419 \\
\hline
\end{tabular}




\begin{tabular}{rrrrrr}
56 & 1 & 0 & -5.254245 & 3.083211 & -0.942929 \\
57 & 1 & 0 & -2.942282 & 2.912797 & -0.071076 \\
58 & 1 & 0 & -5.479743 & -1.218446 & -1.079341 \\
59 & 8 & 0 & -3.125712 & -1.640294 & -0.200392 \\
60 & 6 & 0 & -1.496632 & 0.655679 & 0.435290 \\
61 & 1 & 0 & -0.792495 & 0.940834 & -0.359820 \\
62 & 1 & 0 & -1.354264 & 1.375363 & 1.248197 \\
63 & 6 & 0 & -1.511982 & -3.254294 & 0.152860 \\
64 & 1 & 0 & -2.123583 & -3.805251 & -0.566833 \\
65 & 1 & 0 & -0.468512 & -3.537253 & -0.009881 \\
66 & 1 & 0 & -1.818844 & -3.558312 & 1.154960 \\
67 & 8 & 0 & 0.793237 & 0.290466 & 1.918510 \\
68 & 1 & 0 & 1.070473 & -1.960861 & -0.339381 \\
69 & 1 & 0 & 0.898020 & 1.024576 & 1.242369 \\
\hline
\end{tabular}

(33) $\mathrm{TS}_{12}$
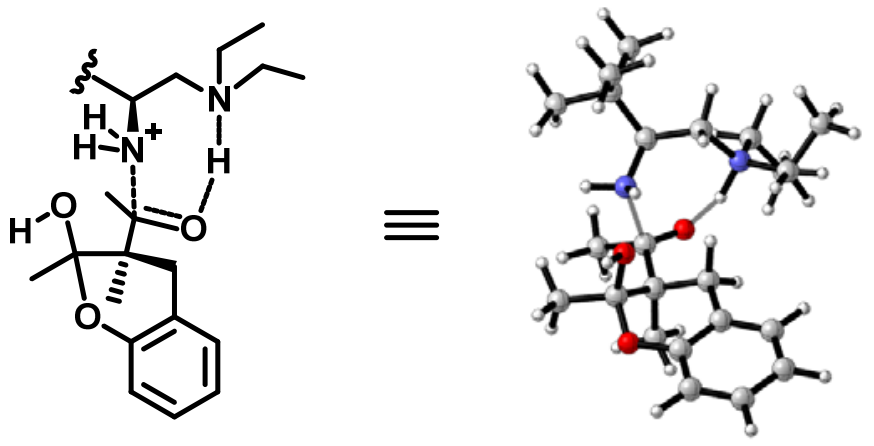

$\mathrm{E}_{\text {sol }}=-1236.013568$

Zero-point correction=

Thermal correction to Energy=

0.628998 (Hartree/Particle)

Thermal correction to Enthalpy=

0.658384

Thermal correction to Gibbs Free Energy=

Sum of electronic and zero-point Energies=

0.659328

0. 572211

Sum of electronic and thermal Energies=

$-1234.932673$

$-1234.903287$

Sum of electronic and thermal Enthalpies=

$-1234.902343$

Sum of electronic and thermal Free Energies=

$-1234.989461$

$\begin{array}{lccc} & \text { E (Thermal) } & \text { CV } & \text { S } \\ & \text { KCal/Mol } & \text { Cal/Mol-Kelvin } & \text { Cal/Mo1-Kelvin } \\ \text { Total } & 413.142 & 118.784 & 183.354 \\ \text { Electronic } & 0.000 & 0.000 & 0.000 \\ \text { Translational } & 0.889 & 2.981 & 43.800 \\ \text { Rotational } & 0.889 & 2.981 & 35.755 \\ \text { Vibrational } & 411.365 & 112.823 & 103.799\end{array}$

Standard orientation: 


\begin{tabular}{|c|c|c|c|c|c|}
\hline \multirow{2}{*}{$\begin{array}{l}\text { Center } \\
\text { Number }\end{array}$} & \multirow{2}{*}{$\begin{array}{l}\text { Atomic } \\
\text { Number }\end{array}$} & \multirow{2}{*}{$\begin{array}{l}\text { Atomic } \\
\text { Type }\end{array}$} & \multicolumn{3}{|c|}{ Coordinates (Angstroms) } \\
\hline & & & X & Y & Z \\
\hline 1 & 6 & 0 & 3.530820 & -1.412602 & -1.039651 \\
\hline 2 & 6 & 0 & 4. 916136 & -0.791243 & -0.809307 \\
\hline 3 & 1 & 0 & 5. 164185 & -0.764285 & 0.257984 \\
\hline 4 & 1 & 0 & 5.678479 & -1.394215 & -1.311004 \\
\hline 5 & 1 & 0 & 5. 003806 & 0.224100 & -1.208279 \\
\hline 6 & 6 & 0 & 3. 571396 & -2.846573 & -0.487371 \\
\hline 7 & 1 & 0 & 3. 651893 & -2.856463 & 0.606462 \\
\hline 8 & 1 & 0 & 2.700677 & -3.443798 & -0.782494 \\
\hline 9 & 1 & 0 & 4. 445485 & -3.368753 & -0.886232 \\
\hline 10 & 6 & 0 & 3. 208521 & -1.453198 & -2.538020 \\
\hline 11 & 1 & 0 & 2. 204758 & -1.853538 & -2.725305 \\
\hline 12 & 1 & 0 & 3.275823 & -0.463345 & -3.002854 \\
\hline 13 & 1 & 0 & 3. 920298 & -2.102262 & -3.056925 \\
\hline 14 & 6 & 0 & 2. 471470 & -0.584042 & -0.257420 \\
\hline 15 & 1 & 0 & 2. 822352 & -0.499828 & 0.779222 \\
\hline 16 & 6 & 0 & 2. 281668 & 0.825568 & -0.844657 \\
\hline 17 & 1 & 0 & 1. 546664 & 0.792271 & -1.657318 \\
\hline 18 & 1 & 0 & 3. 217966 & 1. 198443 & -1.264626 \\
\hline 19 & 7 & 0 & 1. 156807 & -1.254140 & -0.172533 \\
\hline 20 & 1 & 0 & -1.804660 & -2.016480 & -1.923292 \\
\hline 21 & 7 & 0 & 1. 799739 & 1. 821626 & 0.159136 \\
\hline 22 & 6 & 0 & 1. 074135 & 2. 972069 & -0.467354 \\
\hline 23 & 1 & 0 & 0.303378 & 2. 539340 & -1.109692 \\
\hline 24 & 1 & 0 & 0.563241 & 3. 496680 & 0.343168 \\
\hline 25 & 6 & 0 & 2. 908392 & 2. 251098 & 1. 073167 \\
\hline 26 & 1 & 0 & 3. 333998 & 1. 334530 & 1. 485704 \\
\hline 27 & 1 & 0 & 3. 676337 & 2. 742903 & 0.468806 \\
\hline 28 & 6 & 0 & 2. 402543 & 3. 132499 & 2. 202617 \\
\hline 29 & 1 & 0 & 2. 079075 & 4. 116214 & 1. 852567 \\
\hline 30 & 1 & 0 & 1. 576308 & 2. 640661 & 2. 724620 \\
\hline 31 & 1 & 0 & 3.210721 & 3. 287298 & 2. 920794 \\
\hline 32 & 6 & 0 & 1.970444 & 3. 913215 & -1.256213 \\
\hline 33 & 1 & 0 & 2.521380 & 3. 391436 & -2.044292 \\
\hline 34 & 1 & 0 & 1. 346528 & 4. 669330 & -1.738278 \\
\hline 35 & 1 & 0 & 2. 683830 & 4. 436846 & -0.614917 \\
\hline 36 & 1 & 0 & 0.547543 & -1.076666 & -0.976248 \\
\hline 37 & 6 & 0 & 0.242705 & -0.824394 & 1. 434338 \\
\hline 38 & 6 & 0 & -1.253005 & -0.725417 & 0.994592 \\
\hline 39 & 6 & 0 & 0.668643 & -2.019172 & 2. 278981 \\
\hline 40 & 1 & 0 & 1. 761589 & -2.042519 & 2. 326708 \\
\hline 41 & 1 & 0 & 0.310232 & -1.846023 & 3. 297070 \\
\hline
\end{tabular}




\begin{tabular}{|c|c|c|c|c|c|}
\hline 42 & 1 & 0 & 0.293722 & -2.984173 & 1. 938910 \\
\hline 43 & 6 & 0 & -2.049869 & -0.774532 & 2. 315278 \\
\hline 44 & 1 & 0 & -2.048436 & -1.771801 & 2. 762853 \\
\hline 45 & 1 & 0 & -1.603323 & -0.076836 & 3. 029085 \\
\hline 46 & 1 & 0 & -3.088198 & -0.480935 & 2. 149006 \\
\hline 47 & 6 & 0 & -1.838776 & -1.782749 & 0.006759 \\
\hline 48 & 8 & 0 & -1.270168 & -1.530882 & -1.274060 \\
\hline 49 & 6 & 0 & -5.504050 & 1. 036570 & -1.151748 \\
\hline 50 & 6 & 0 & -4.719378 & 2. 176104 & -0.974843 \\
\hline 51 & 6 & 0 & -3.421212 & 2. 047164 & -0.497267 \\
\hline 52 & 6 & 0 & -2.878836 & 0.795969 & -0.198670 \\
\hline 53 & 6 & 0 & -3.680901 & -0.326925 & -0.391869 \\
\hline 54 & 6 & 0 & -4.987260 & -0.218596 & -0.860240 \\
\hline 55 & 1 & 0 & -6.522100 & 1. 125904 & -1.516184 \\
\hline 56 & 1 & 0 & -5.123067 & 3. 158054 & -1.197078 \\
\hline 57 & 1 & 0 & -2.810963 & 2. 933910 & -0.336840 \\
\hline 58 & 1 & 0 & -5.574508 & -1.122486 & -0.983875 \\
\hline 59 & 8 & 0 & -3.235192 & -1.598099 & -0.096644 \\
\hline 60 & 6 & 0 & -1.476371 & 0.642757 & 0.329922 \\
\hline 61 & 1 & 0 & -0.763960 & 0.756836 & -0.501116 \\
\hline 62 & 1 & 0 & -1.260214 & 1. 430069 & 1. 059147 \\
\hline 63 & 6 & 0 & -1.682479 & -3.256044 & 0.352732 \\
\hline 64 & 1 & 0 & -2.338196 & -3.828566 & -0.309381 \\
\hline 65 & 1 & 0 & -0.655956 & -3.587528 & 0.184593 \\
\hline 66 & 1 & 0 & -1.973561 & -3.473914 & 1. 381609 \\
\hline 67 & 8 & 0 & 0.767284 & 0.273618 & 1. 831905 \\
\hline 68 & 1 & 0 & 1. 280016 & -2.266114 & -0.140638 \\
\hline 69 & 1 & 0 & 1. 127229 & 1. 251059 & 0.840191 \\
\hline
\end{tabular}

\section{(34) Int11}
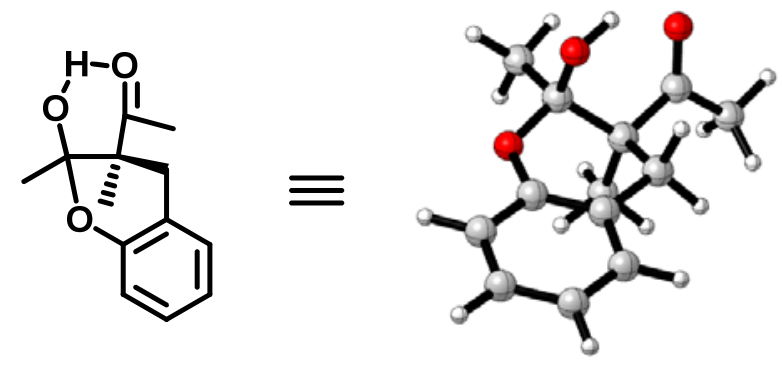

$\mathrm{E}_{\mathrm{sol}}=-730.6278829$

Zero-point correction=

0.270924 (Hartree/Particle)

Thermal correction to Energy=

0.285419

Thermal correction to Enthalpy=

0. 286364

Thermal correction to Gibbs Free Energy=

Sum of electronic and zero-point Energies=

0.230689

$-730.123473$ 
Sum of electronic and thermal Energies=

Sum of electronic and thermal Enthalpies=

Sum of electronic and thermal Free Energies=
$-730.108978$

$-730.108034$

$-730.163709$

E (Therma1)

CV

$\mathrm{S}$

Total

$\mathrm{KCal} / \mathrm{Mol}$

Electronic

179. 103

Cal/Mo1-Kelvin

Cal/Mol-Kelvin

58. 369

117. 177

0.000

0.000

0.000

Translational

0.889

2. 981

42. 070

Rotational

0.889

2. 981

32. 020

Vibrational

177.326

52. 407

43. 088

Standard orientation:

\begin{tabular}{llccc} 
Center & Atomic & Atomic & \multicolumn{2}{c}{ Coordinates (Angstroms) } \\
Number & Number & Type & X & Y
\end{tabular}

\begin{tabular}{|c|c|c|c|c|c|}
\hline 1 & 1 & 0 & -1.572373 & 1. 040709 & -1.828085 \\
\hline 2 & 6 & 0 & -2.512215 & -0.659312 & -0.219352 \\
\hline 3 & 6 & 0 & -1.119084 & -0.288885 & 0.292402 \\
\hline 4 & 6 & 0 & -3.235757 & -1.807411 & 0.447293 \\
\hline 5 & 1 & 0 & -4.113636 & -2.065409 & -0.144938 \\
\hline 6 & 1 & 0 & -2.579422 & -2.677845 & 0.547585 \\
\hline 7 & 1 & 0 & -3.547229 & -1.520282 & 1. 456977 \\
\hline 8 & 6 & 0 & -1.004326 & -0.485715 & 1. 809444 \\
\hline 9 & 1 & 0 & -1.785701 & 0.058831 & 2.348608 \\
\hline 10 & 1 & 0 & -1.084019 & -1.543928 & 2. 075522 \\
\hline 11 & 1 & 0 & -0.036239 & -0.124791 & 2. 164129 \\
\hline 12 & 6 & 0 & -0.748127 & 1. 173592 & -0.106943 \\
\hline 13 & 8 & 0 & -0.691564 & 1. 299217 & -1.502096 \\
\hline 14 & 6 & 0 & 3. 915756 & 0.158790 & 0.131132 \\
\hline 15 & 6 & 0 & 3. 683916 & -1.167301 & -0.232878 \\
\hline 16 & 6 & 0 & 2. 377935 & -1.606833 & -0.411209 \\
\hline 17 & 6 & 0 & 1. 291440 & -0.747900 & -0.241658 \\
\hline 18 & 6 & 0 & 1.545602 & 0.577031 & 0.119468 \\
\hline 19 & 6 & 0 & 2.850307 & 1. 030056 & 0.309312 \\
\hline 20 & 1 & 0 & 4. 930357 & 0.516502 & 0.276607 \\
\hline 21 & 1 & 0 & 4. 513045 & -1.853103 & -0.372371 \\
\hline 22 & 1 & 0 & 2. 184641 & -2.641023 & -0.687838 \\
\hline 23 & 1 & 0 & 2. 999484 & 2. 066492 & 0.593085 \\
\hline 24 & 8 & 0 & 0.545959 & 1. 477649 & 0.369827 \\
\hline 25 & 6 & 0 & -0.126277 & -1.211993 & -0.453552 \\
\hline 26 & 1 & 0 & -0.358365 & -1.192394 & -1.524434 \\
\hline 27 & 1 & 0 & -0.238989 & -2.246074 & -0.105769 \\
\hline 28 & 6 & 0 & -1.652722 & 2. 233572 & 0.504685 \\
\hline
\end{tabular}




\begin{tabular}{rrrrrr}
29 & 1 & 0 & -1.303619 & 3.207597 & 0.155877 \\
30 & 1 & 0 & -2.685133 & 2.088550 & 0.177901 \\
31 & 1 & 0 & -1.606449 & 2.213214 & 1.595802 \\
32 & 8 & 0 & -3.018775 & -0.091016 & -1.169921 \\
\hline
\end{tabular}

\section{Reference:}

(1) Perrin, D. D.; Armarego, W. L. F. Purification of Laboratory Chemicals; $3^{\text {rd }}$ ed., Pergamon Press, Oxford, 1988.

(2) Pangborn, A. B.; Giardello, M. A.; Grubbs, R. H.; Rosen, R. K.; Timmers, F. J. Organometallics, 1996, 15, 1518.

(3) Izquierdo, J.; Orue, A.; Scheidt, K. A. J. Am. Chem. Soc. 2013, 135, 10634.

(4) (a) Song, X.-G.; Zhu, S.-F.; Xie, X.-L.; Zhou, Q.-L. Angew. Chem. Int. Ed. 2013, 52, 2555. (b) Carrillo, R.; Hynes, M. J.; Martín, V. S.; Martín, T.; Crióstomo, F. P. Org. Lett. 2015, 17, 2912. (c) Lewis, R. S.; Garza, C. J.; Dang, A. T.; Pedro, T. K. A.; Chain, W. J. Org. Lett. 2015, 17, 2278.

(5) Kalaitzakis, D.; Rozzell, J. D.; Smonou, I.; Kambourakis, S. Adv. Synth. Catal. 2006, 348, 1958.

(6) Sui, X.; Zhu, R.; Li, G.; Ma, G.; Gu, Z. J. Am. Chem. Soc. 2013, 135, 9138.

(7) Moriaty, R. M.; Vaid, R. K.; Ravikumar, V. T.; Hopkins, T. E. Tetrahedron. 1988, 44, 1603.

(8) Ahmed, Z.; Langer, P. Tetrahedron Letters. 2oo6, 47, 417.

(9) Anna K. B.; Soren, B.; Rabinder Kumar, P. UK. Patent 2013067302, May 10, 2013.

(10) Kopp, F.; Stratton, C. F.; Akella, L. B.; Tan, D. S. Nat. Chem. Biol. 2o12, 8, 358.

(11) Brenna, E.; Cosi, S. L.; Ferrandi, E. E.; Gatti, F. G.; Monti, D.; Parmeggiani, F.; Sacchetti, A. Org. Biomol. Chem. 2013, 11, 2988.

(12) Mangas-Sanchez, J.; Busto, E.; Gotor-Fernandez, V.; Gotor, V. Org. Lett. 2010, 12, 3498.

(13) Zhang, L.; Zhang, W.; Liu, J.; Hu, J. J. Org. Chem. 2oo9, 74, 2850. 
(14) Frisch, M. J.; Trucks, G. W.; Schlegel, H. B.; Scuseria, G. E.; Robb, M. A.; Cheeseman, J. R.; Scalmani, G.; Barone, V.; Mennucci, B.; Petersson, G. A.; Nakatsuji, H.; Caricato, M.; Li, X.; Hratchian, H. P.; Izmaylov, A. F.; Bloino, J.; Zheng, G.; Sonnenberg, J. L.; Hada, M.; Ehara, M.; Toyota, K.; Fukuda, R.; Hasegawa, J.; Ishida, M.; Nakajima, T.; Honda, Y.; Kitao, O.; Nakai, H.; Vreven, T.; Montgomery, J. A.; Peralta, Jr., J. E.; Ogliaro, F.; Bearpark, M.; Heyd, J. J.; Brothers, E.; Kudin, K. N.; Staroverov, V. N.; Kobayashi, R.; Normand, J.; Raghavachari, K.; Rendell, A.; Burant, J. C.; Iyengar, S. S.; Tomasi, J.; Cossi, M.; Rega, N.; Millam, J. M.; Klene, M.; Knox, J. E.; Cross, J. B.; Bakken, V.; Adamo, C.; Jaramillo, J.; Gomperts, R.; Stratmann, R. E.; Yazyev, O.; Austin, A. J.; Cammi, R.; Pomelli, C.; Ochterski, J. W.; Martin, R. L.; Morokuma, K.; Zakrzewski, V. G.; Voth, G. A.; Salvador, P.; Dannenberg, J. J.; Dapprich, S.; Daniels, A. D.; Farkas, O.; Foresman, J. B.; Ortiz, J. V.; Cioslowski, J.; Fox, D. J. Gaussian o9, Revision A.o1, Gaussian, Inc., Wallingford CT, 2009.

(15) (a) Zhao, Y.; Truhlar, D. G. Acc. Chem. Res. 2oo8, 41, 157. (b) Zhao, Y.; Truhlar, D. G. Theor. Chem. Acc. 2008, 120, 215.

(16) (a) Marenich, A. V.; Cramer, C. J.; Truhlar, D. G. J. Phys. Chem. B 2oog, 113, 6378. (b) Ribeiro, R. F.; Marenich, A. V.; Cramer, C. J.; Truhlar, D. G. J. Phys. Chem. B 2011, 115, 14556.

(17) (a) Zhang, L.; Fu, N.; Luo, S. Acc. Chem. Res. 2015, 48, 986. (b) Xu, C.; Zhang, L.; Luo, S. Angew. Chem. Int. Ed. 2014, 53, 4149. (c) Zhang, L.; Xu, C.; Mi, X.; Luo, S. Chem. Asian. J. 2014, 9, 3565. (d) Xu, C.; Zhang, L.; Luo, S. J. Org. Chem. 2014, 79, 11517. 
NMR spectral for new products

굴

risisisin

$\stackrel{18}{i}$

ขูल

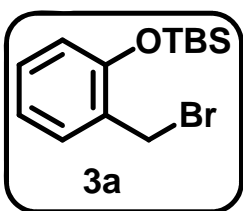

lid du

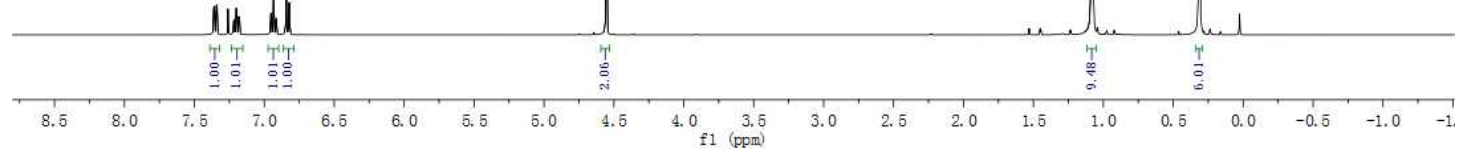

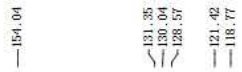

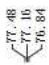
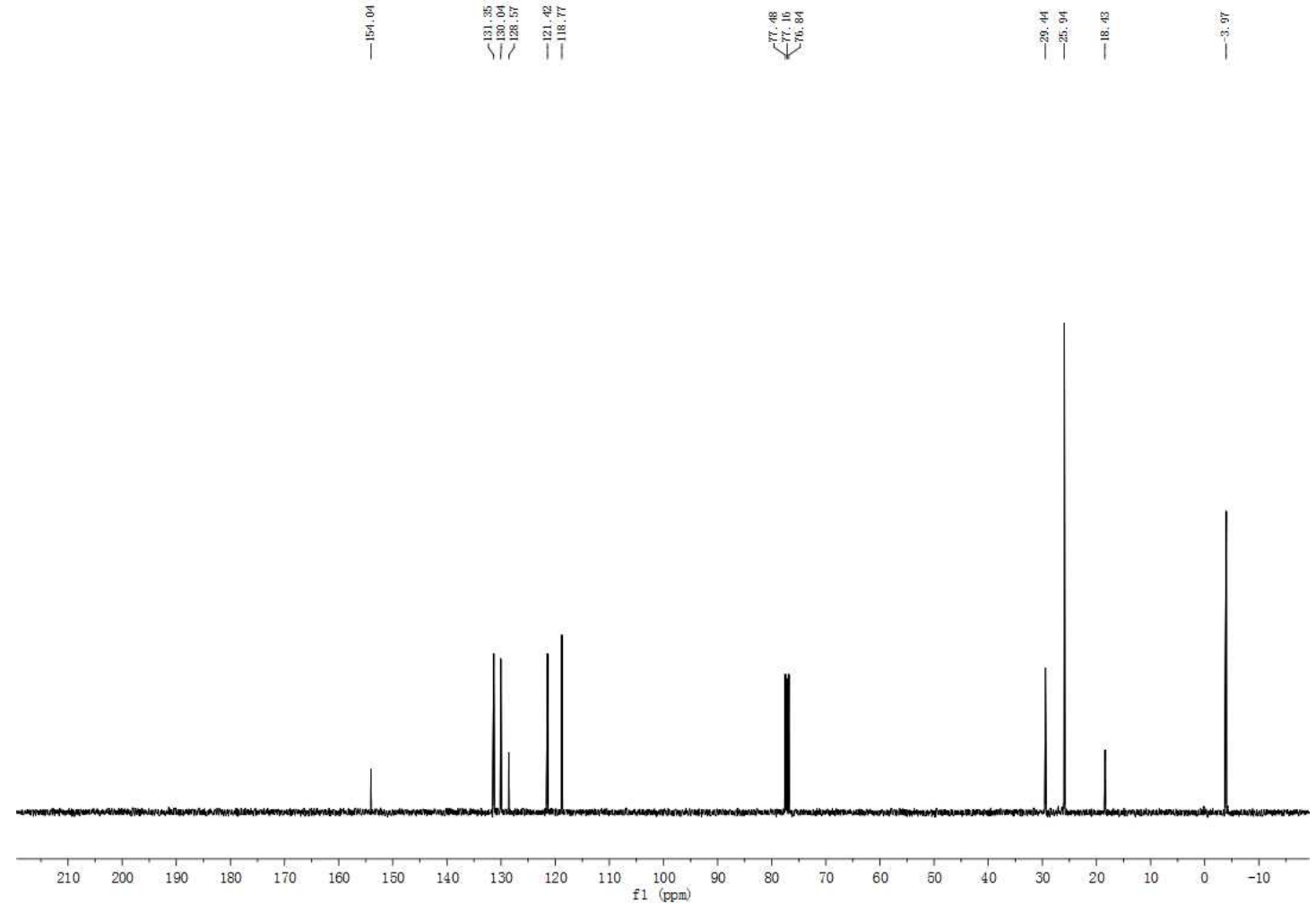

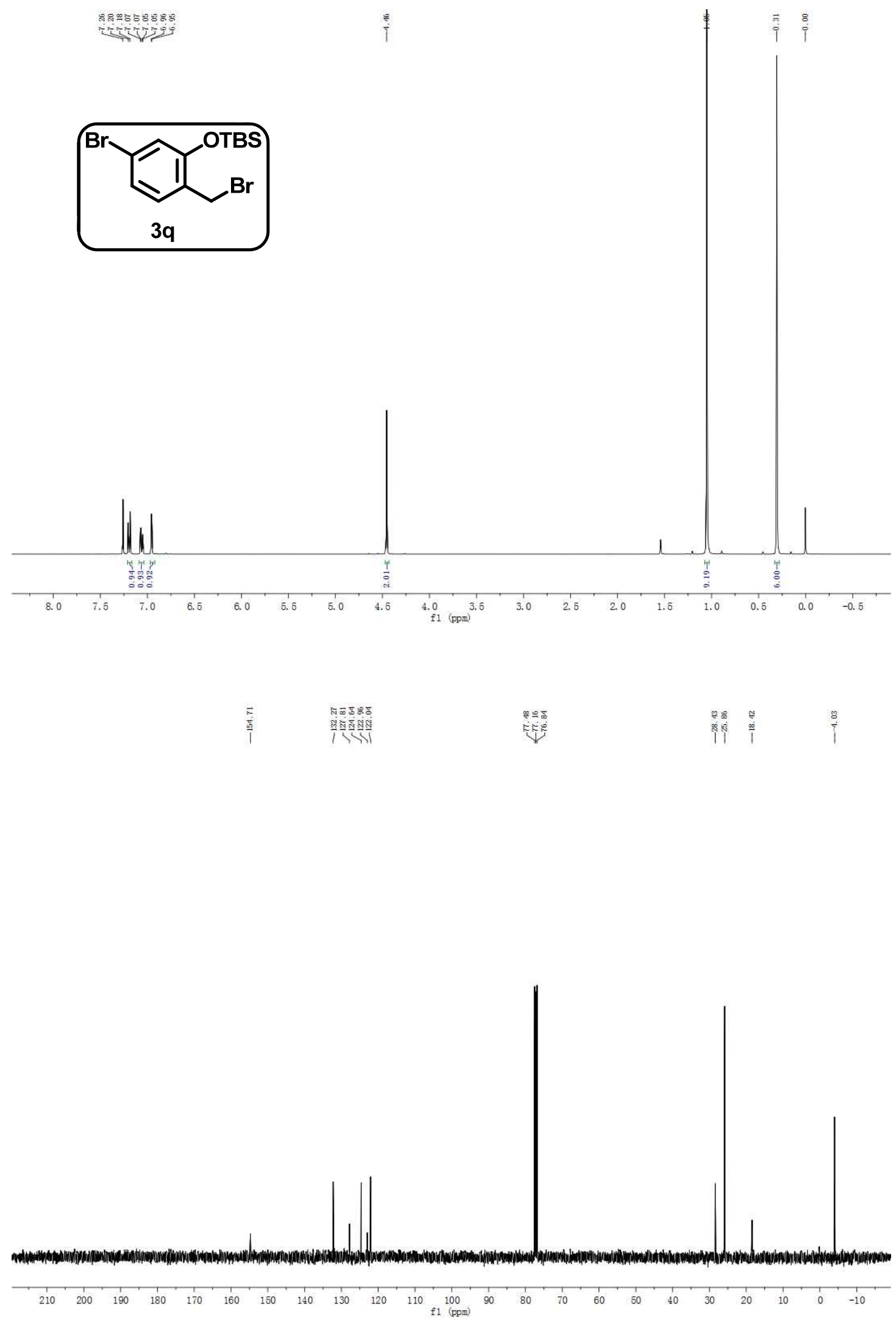

î
i
$\stackrel{8}{i} \frac{8}{p}$
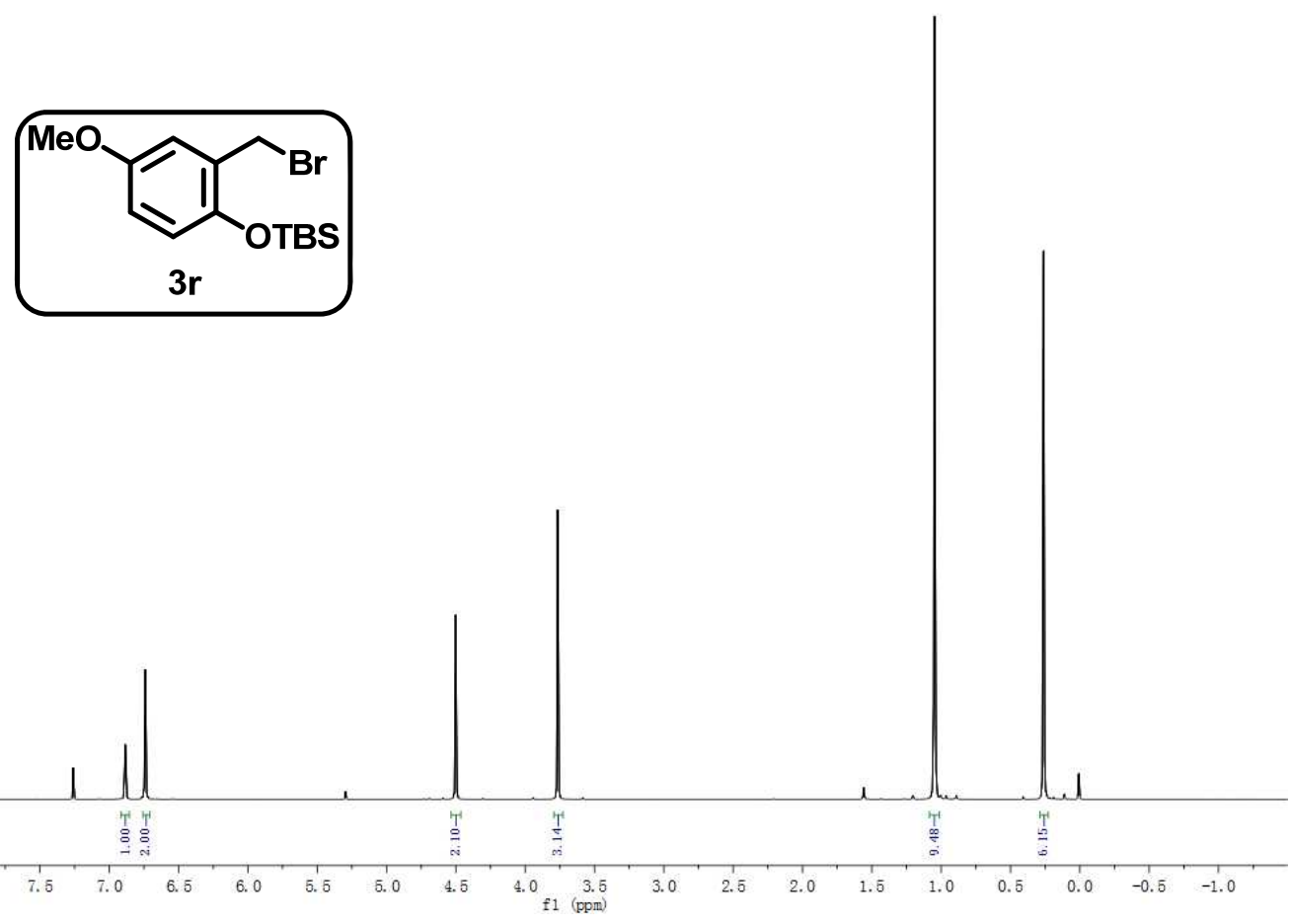

\section{0}

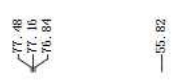

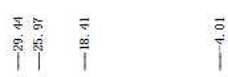

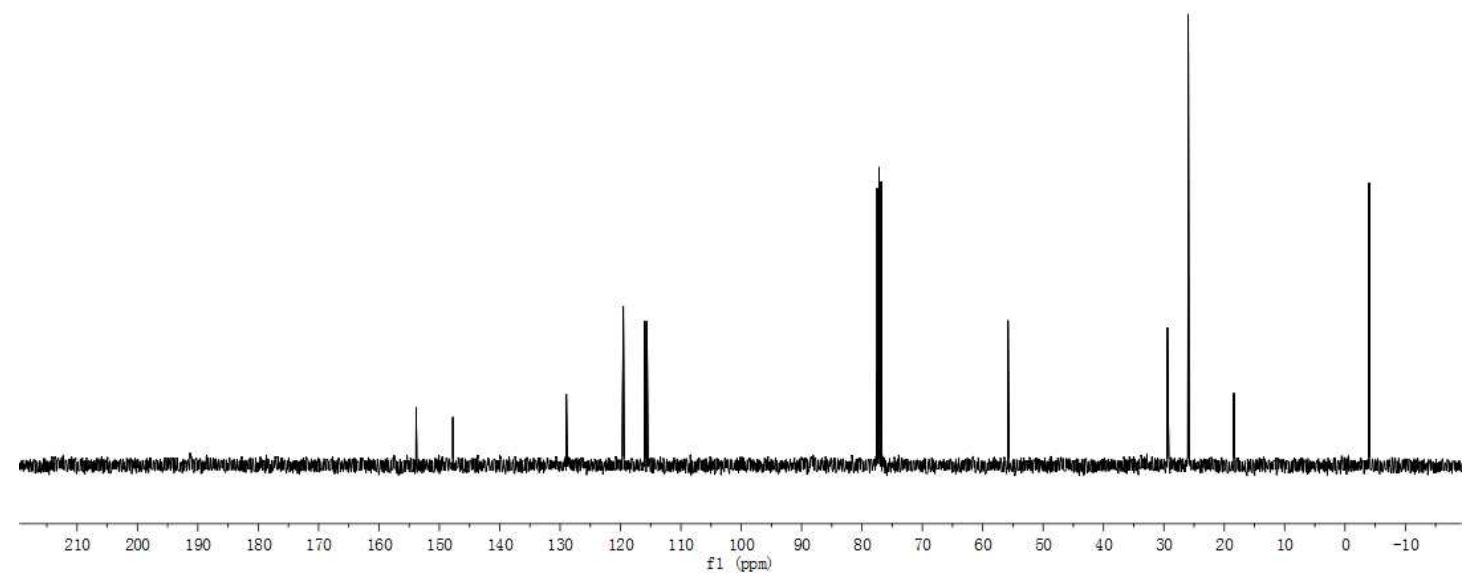



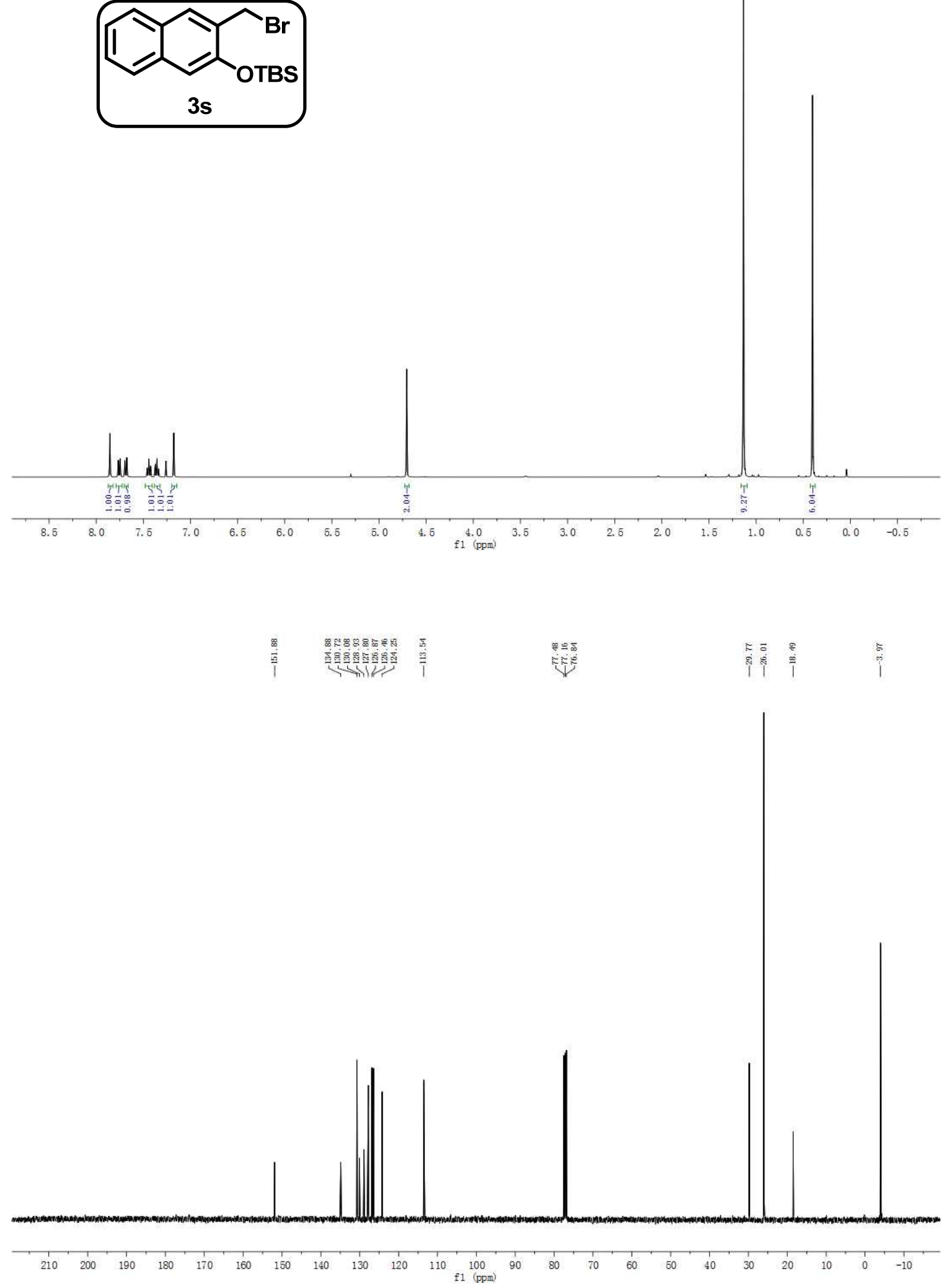


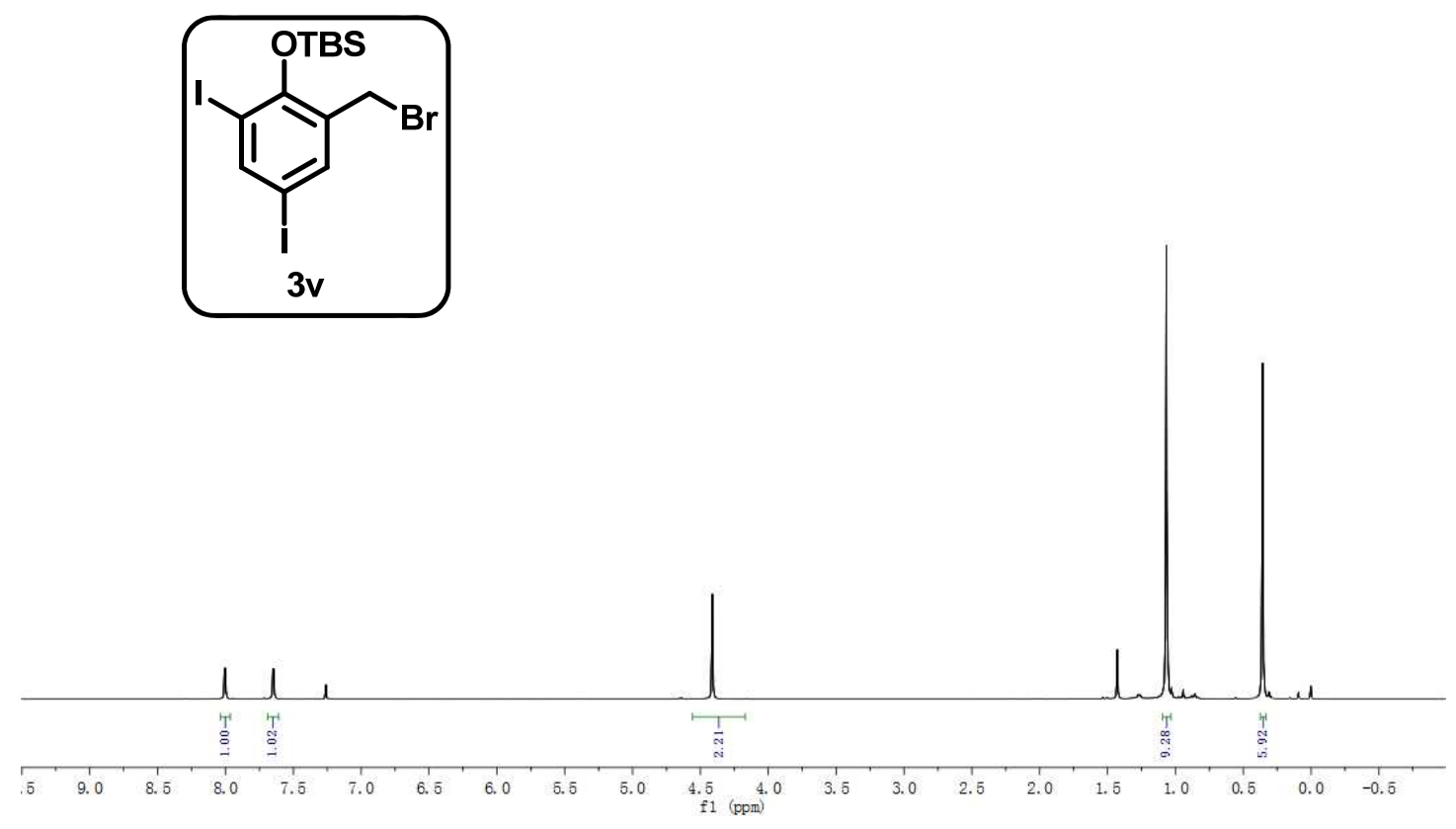

\section{9}

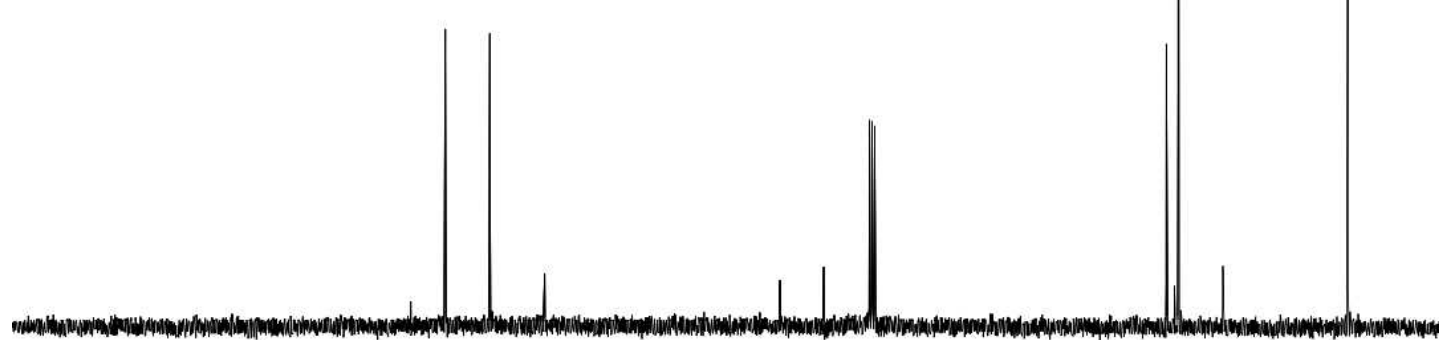

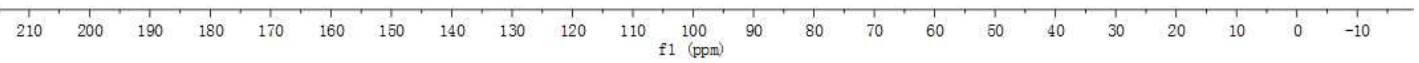




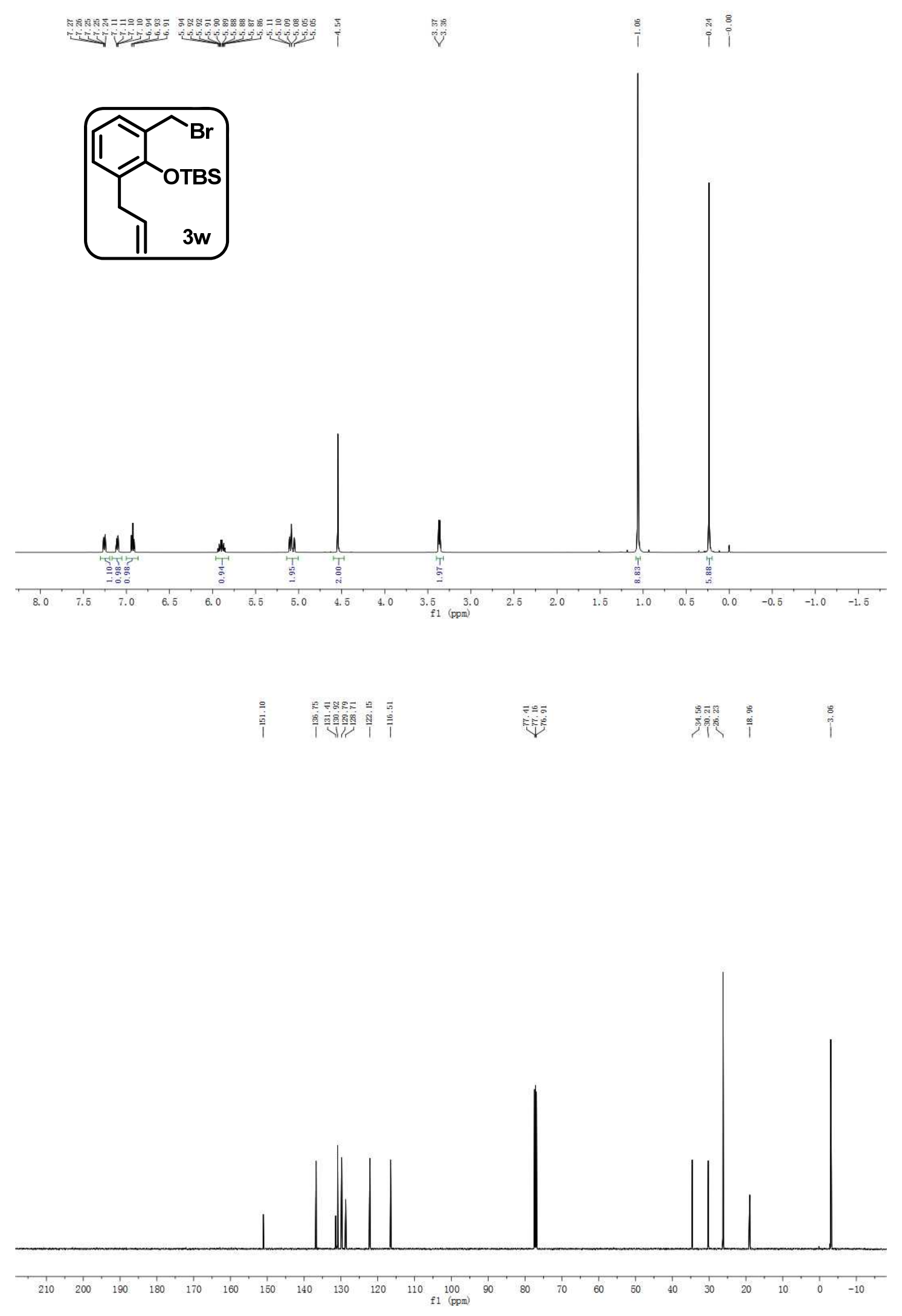



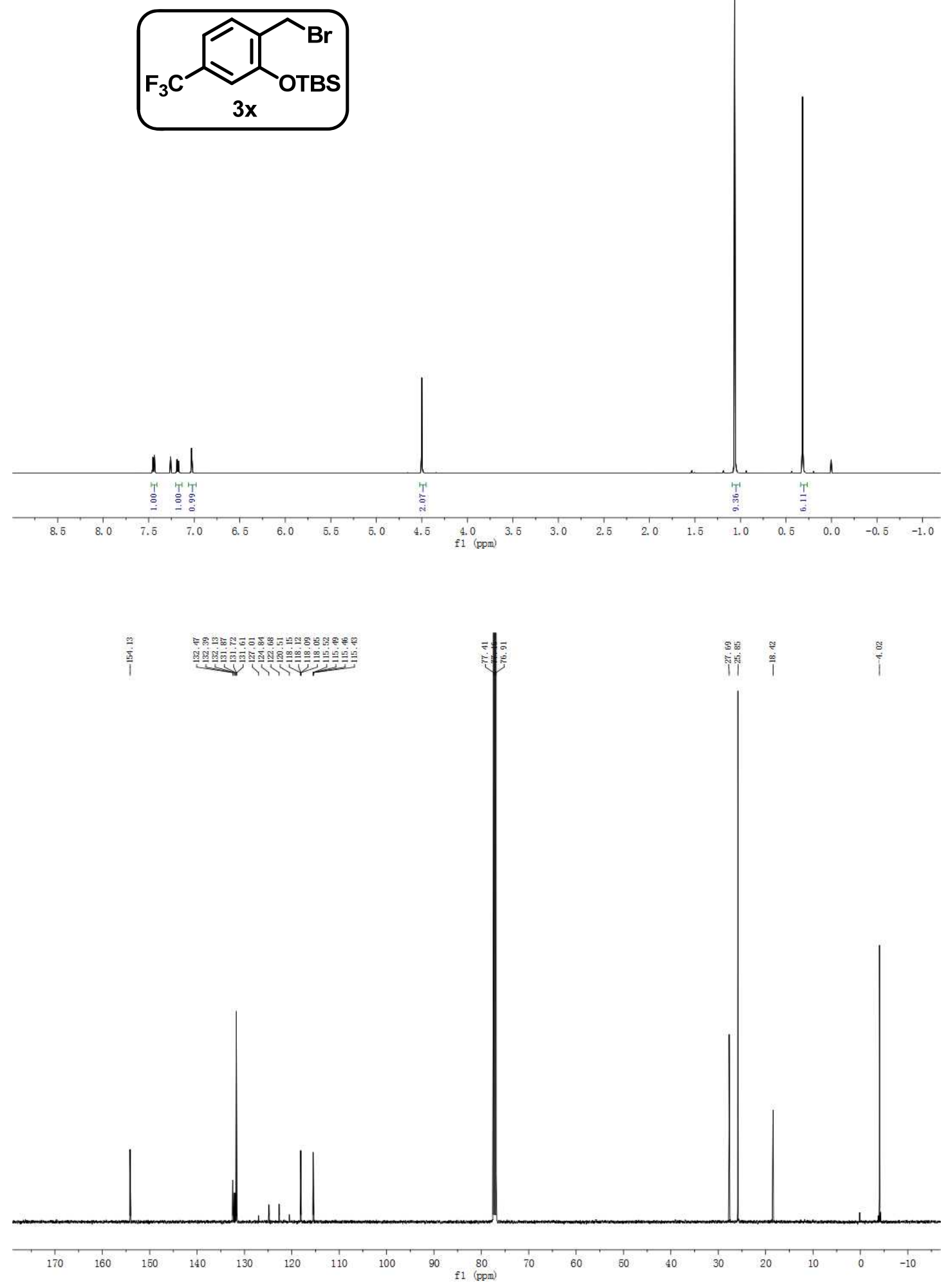


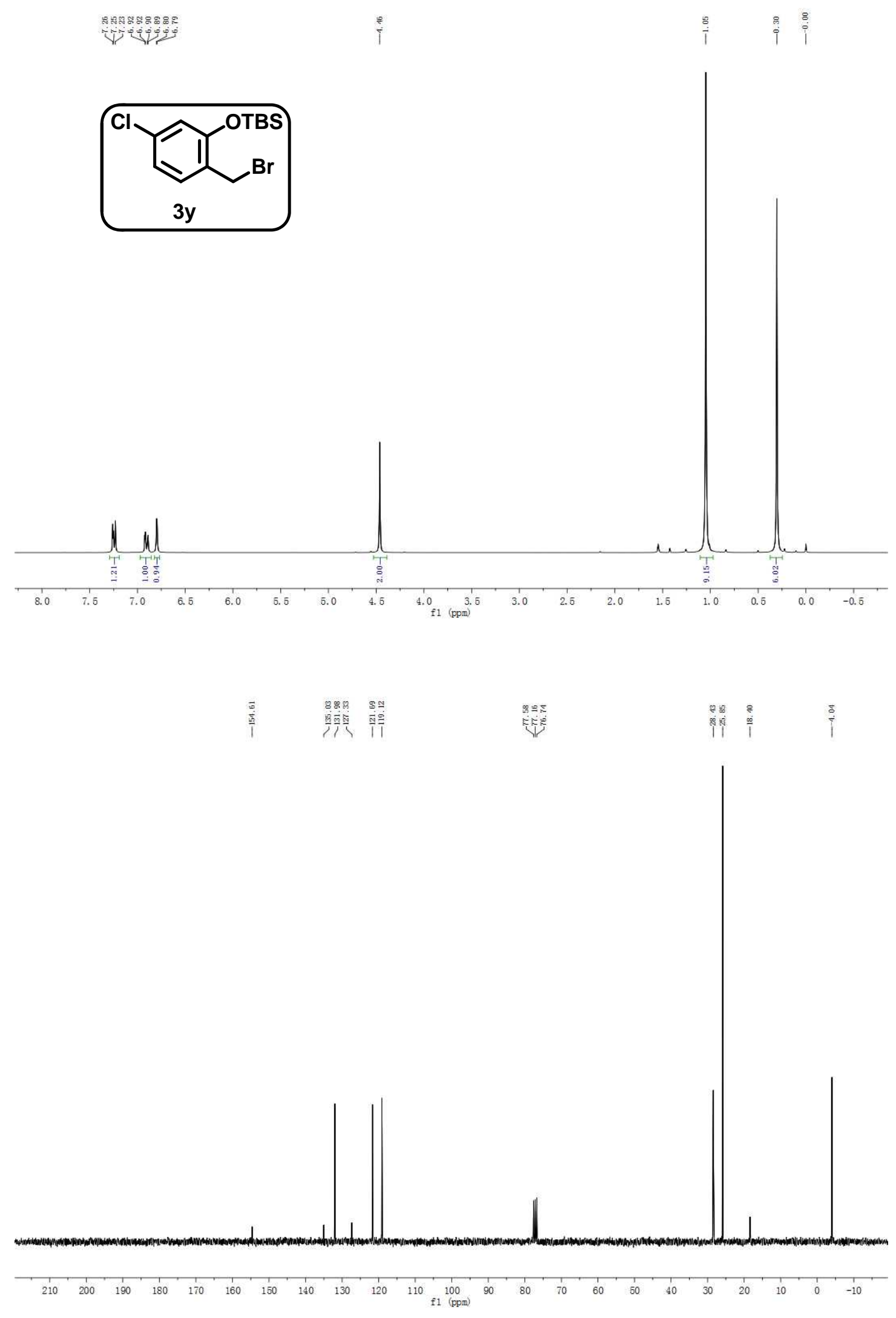



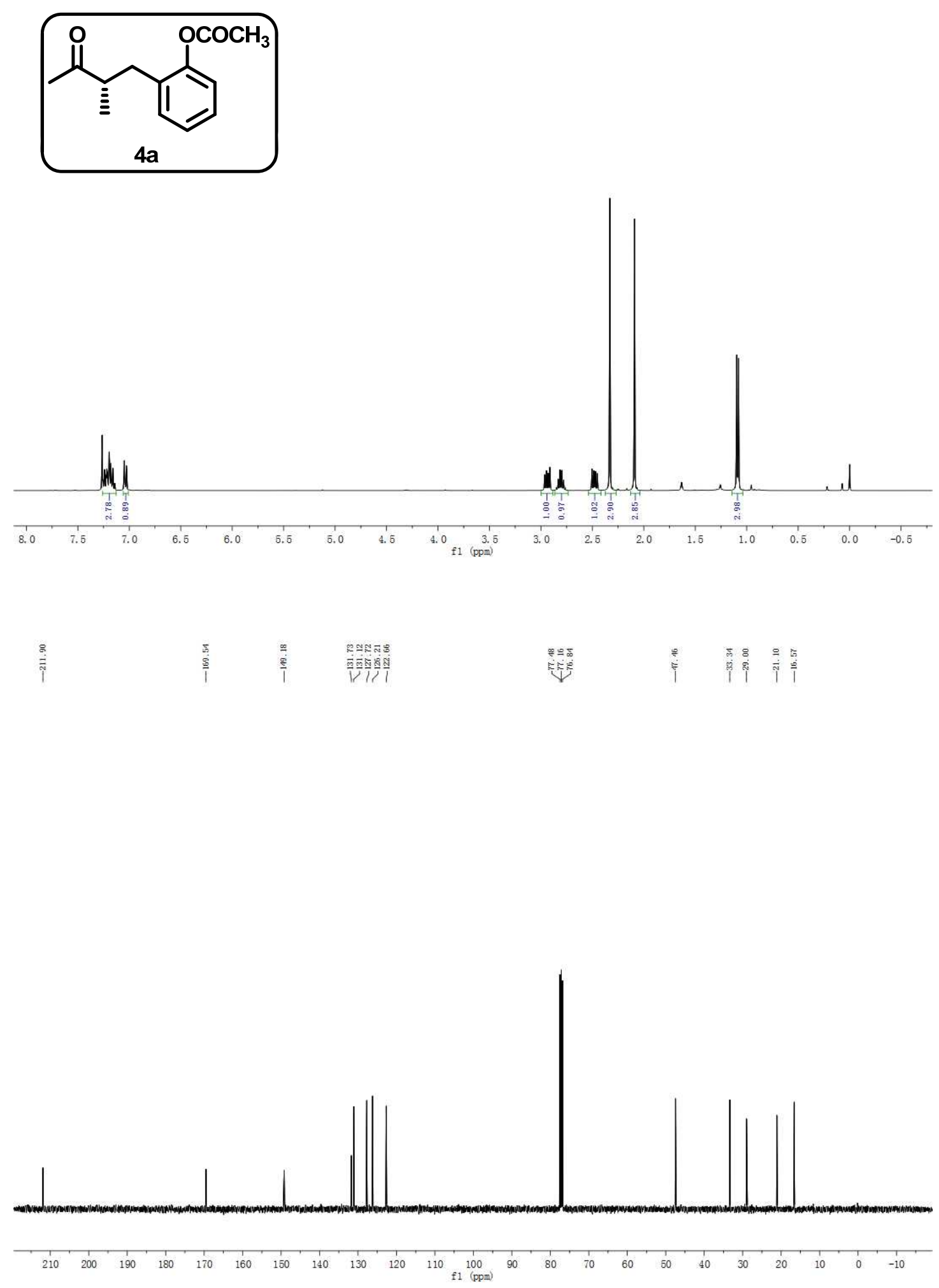

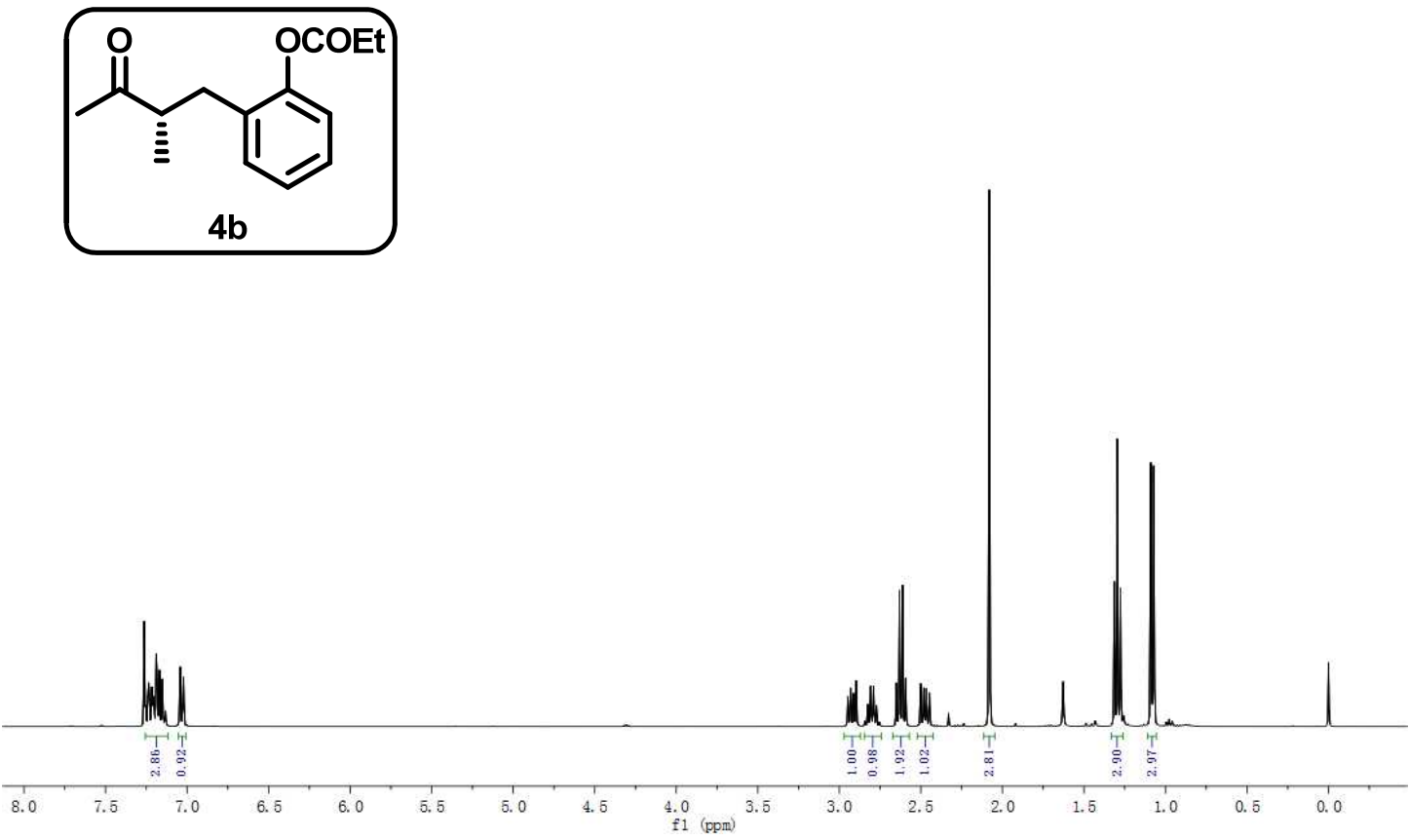

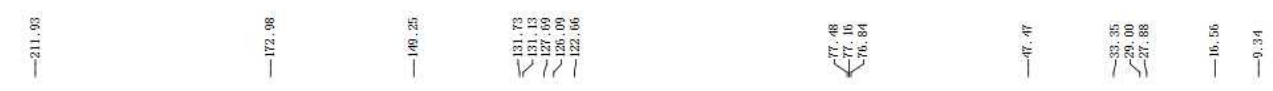

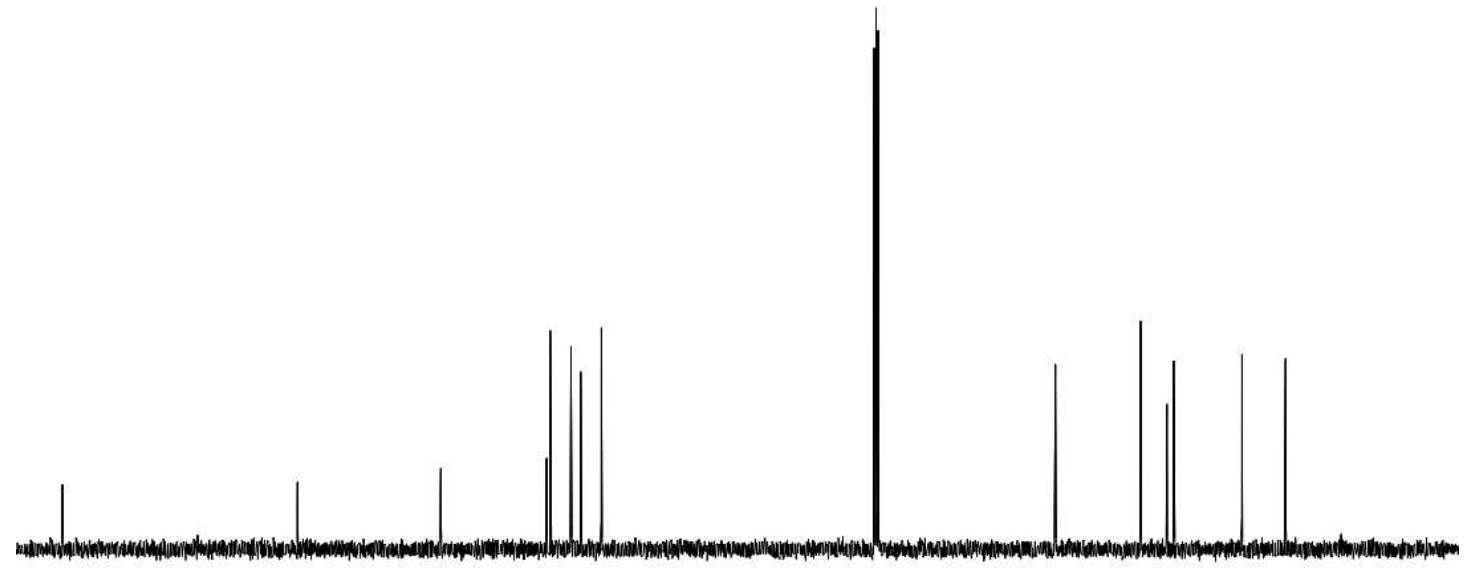

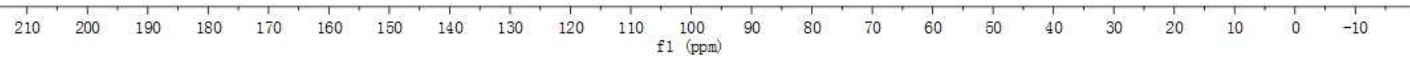



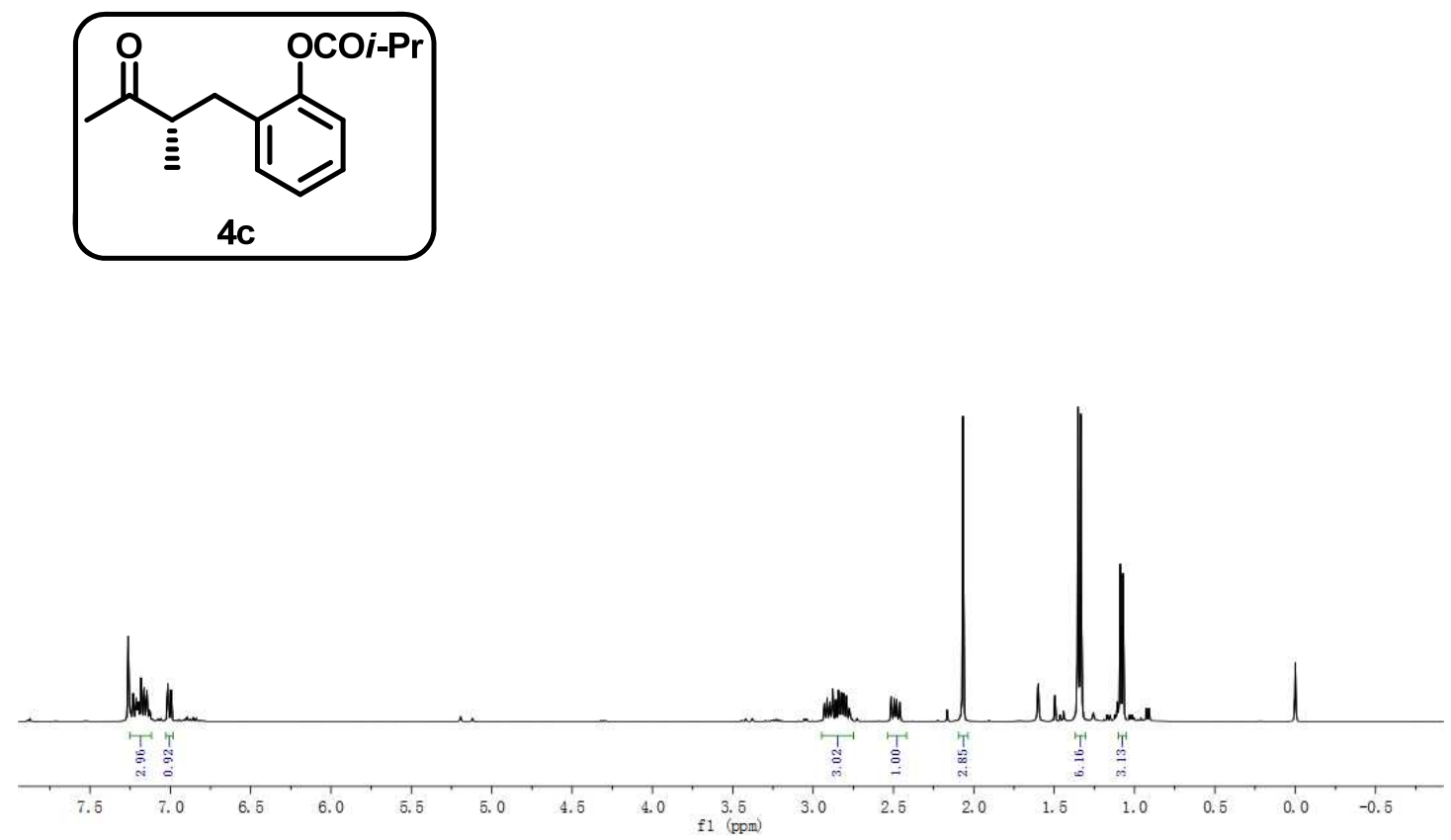

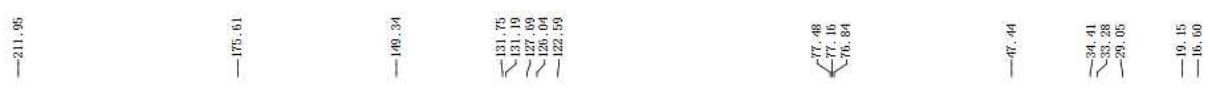

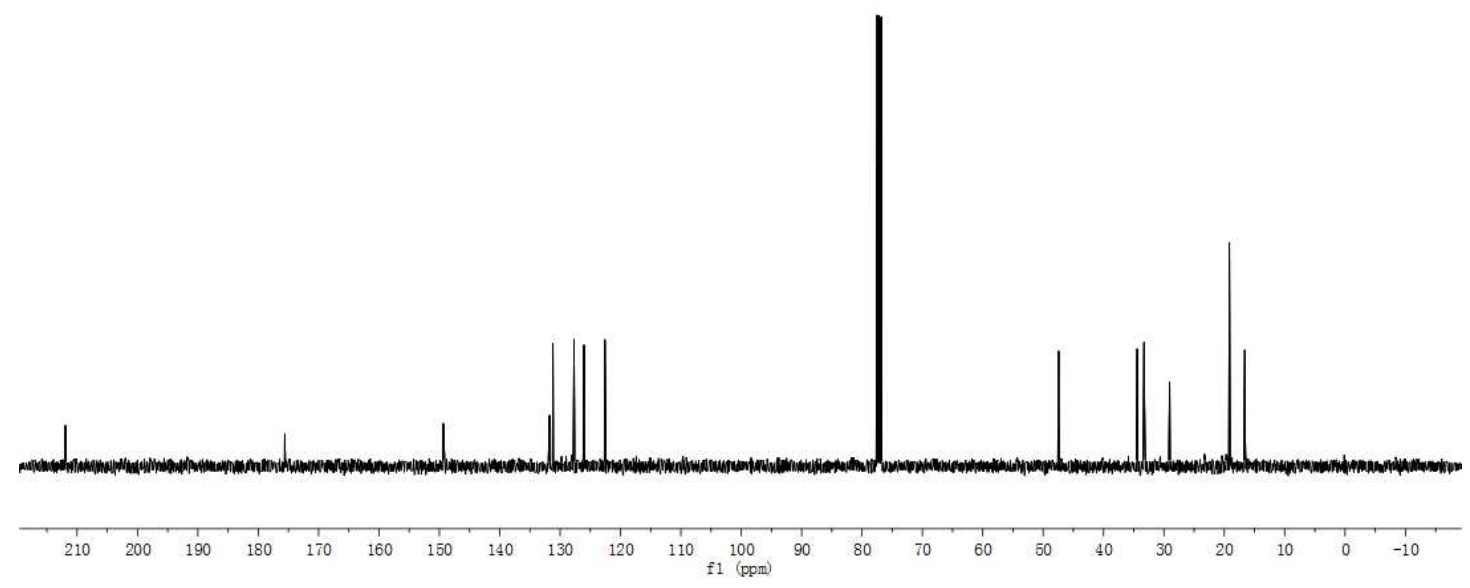



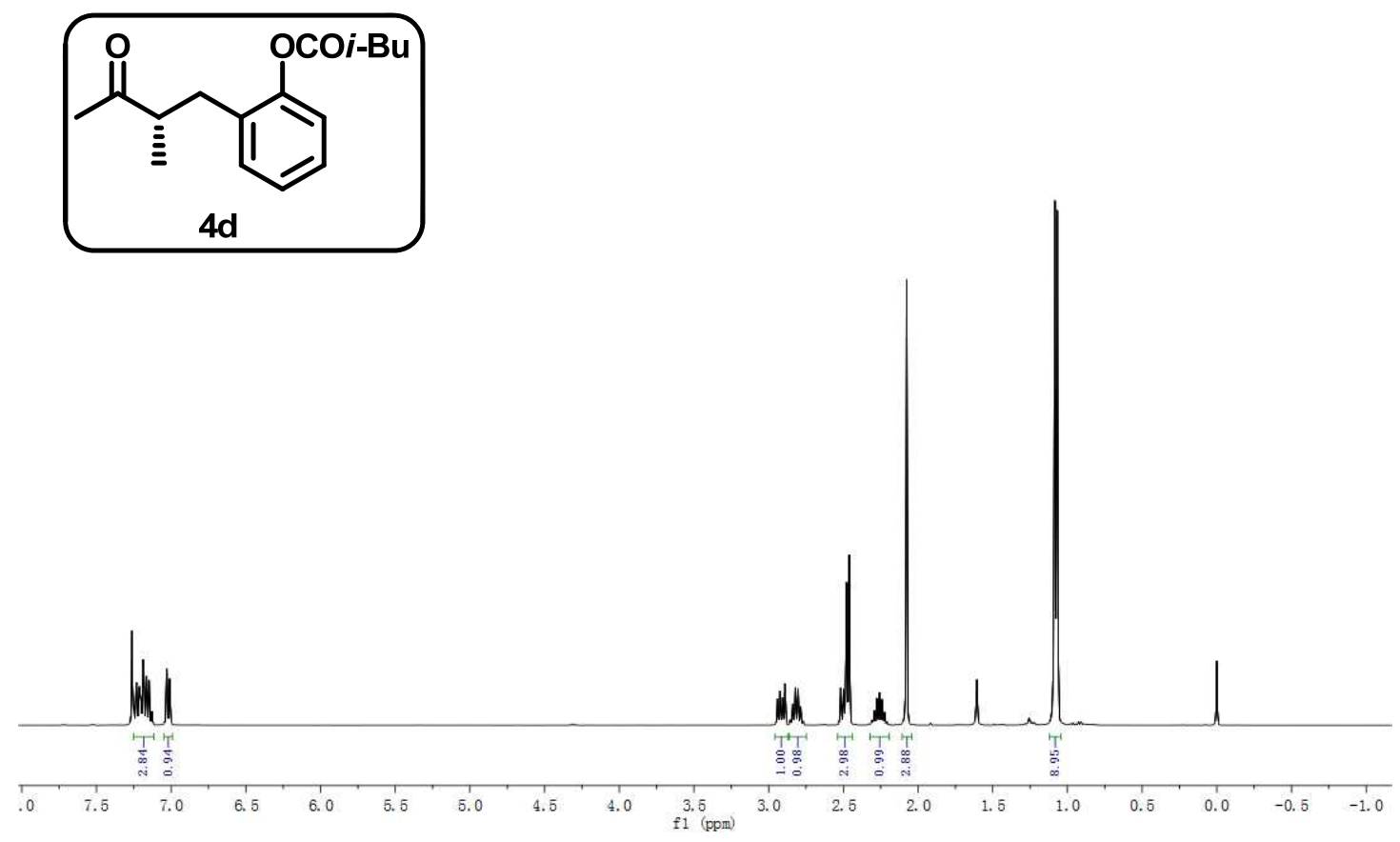

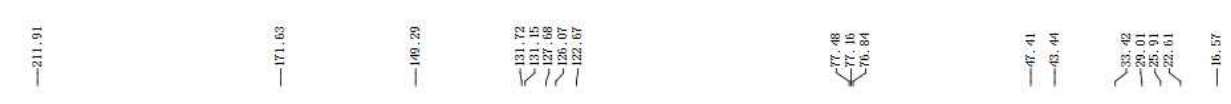

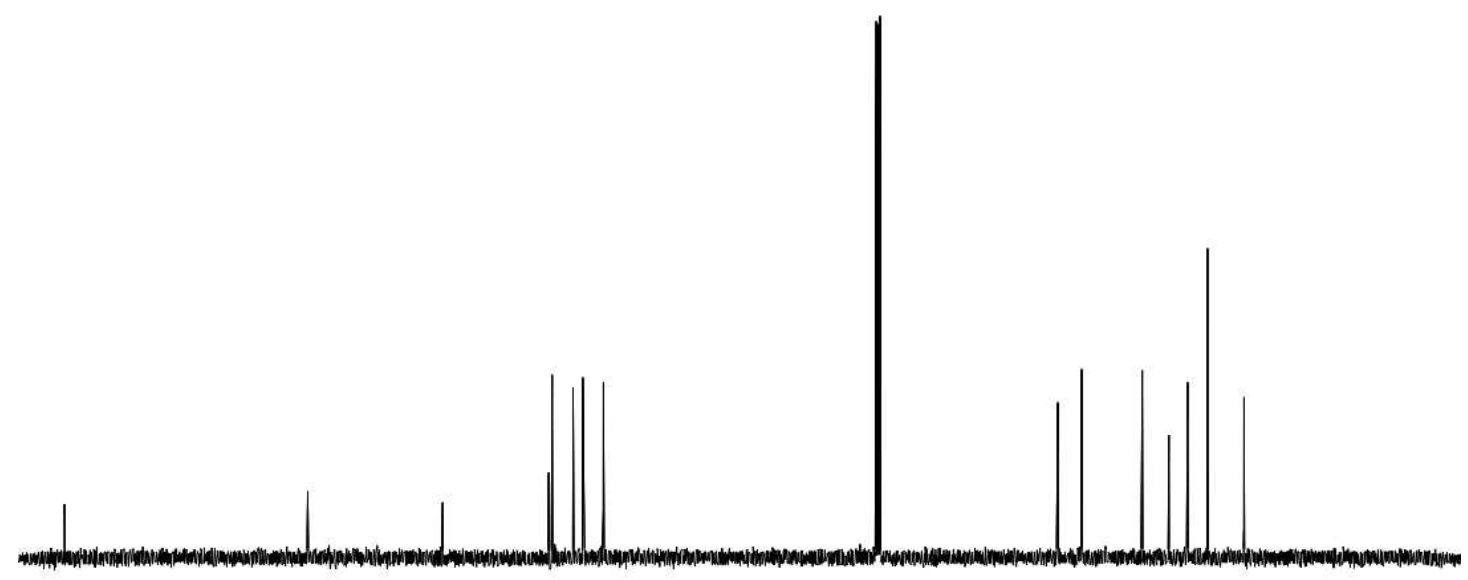

$\begin{array}{llllllllllllllllllllllll}1 & 1 \\ 210 & 200 & 190 & 180 & 170 & 160 & 150 & 140 & 130 & 120 & 110 & 100 & 90 & 80 & 70 & 60 & 50 & 40 & 30 & 20 & 10 & 0 & -10\end{array}$ 

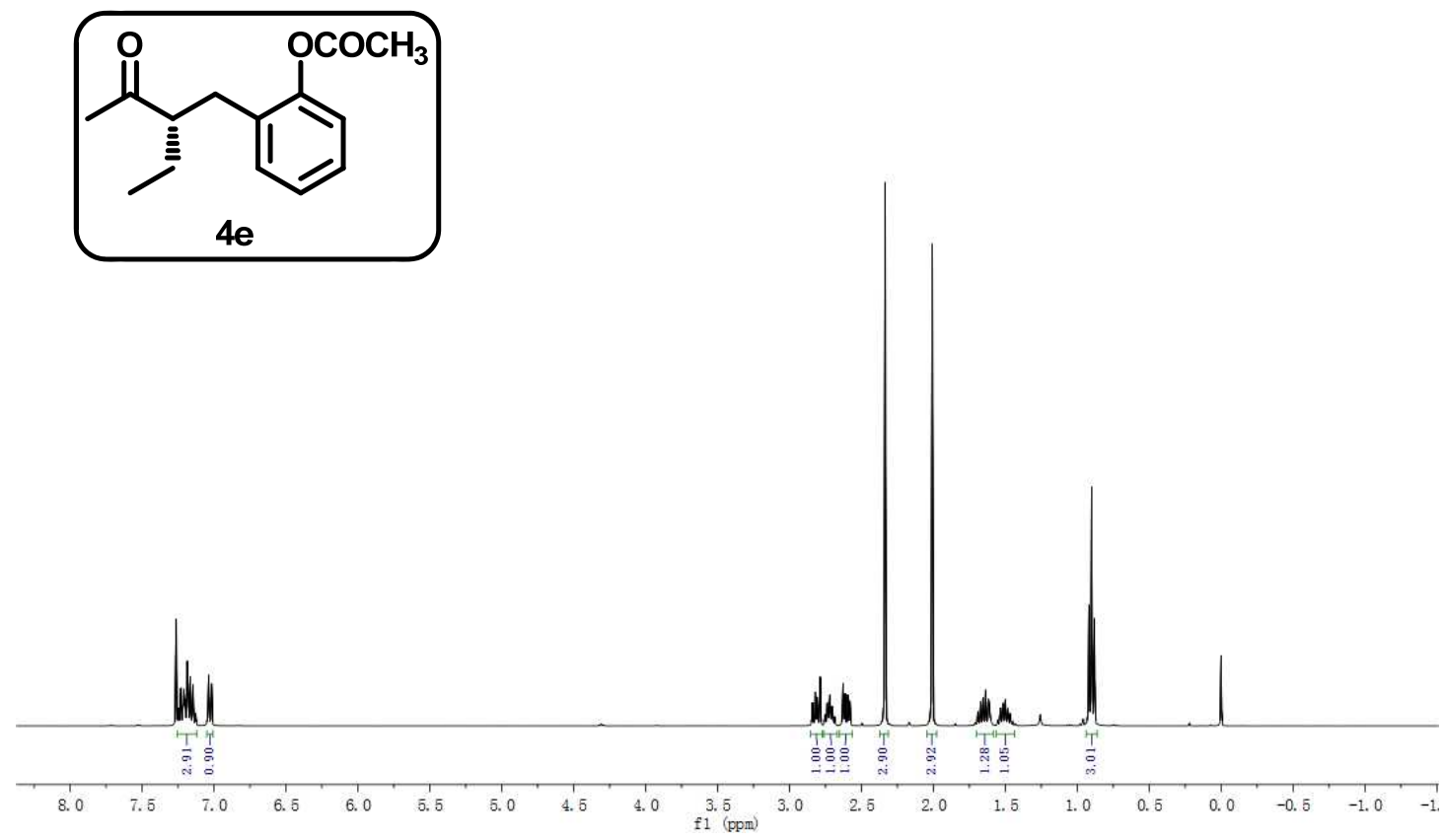

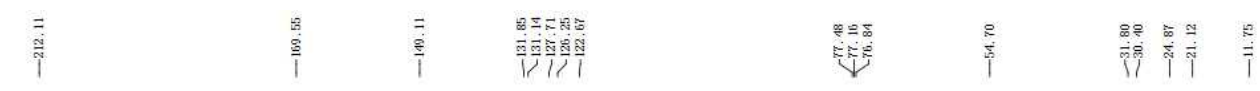

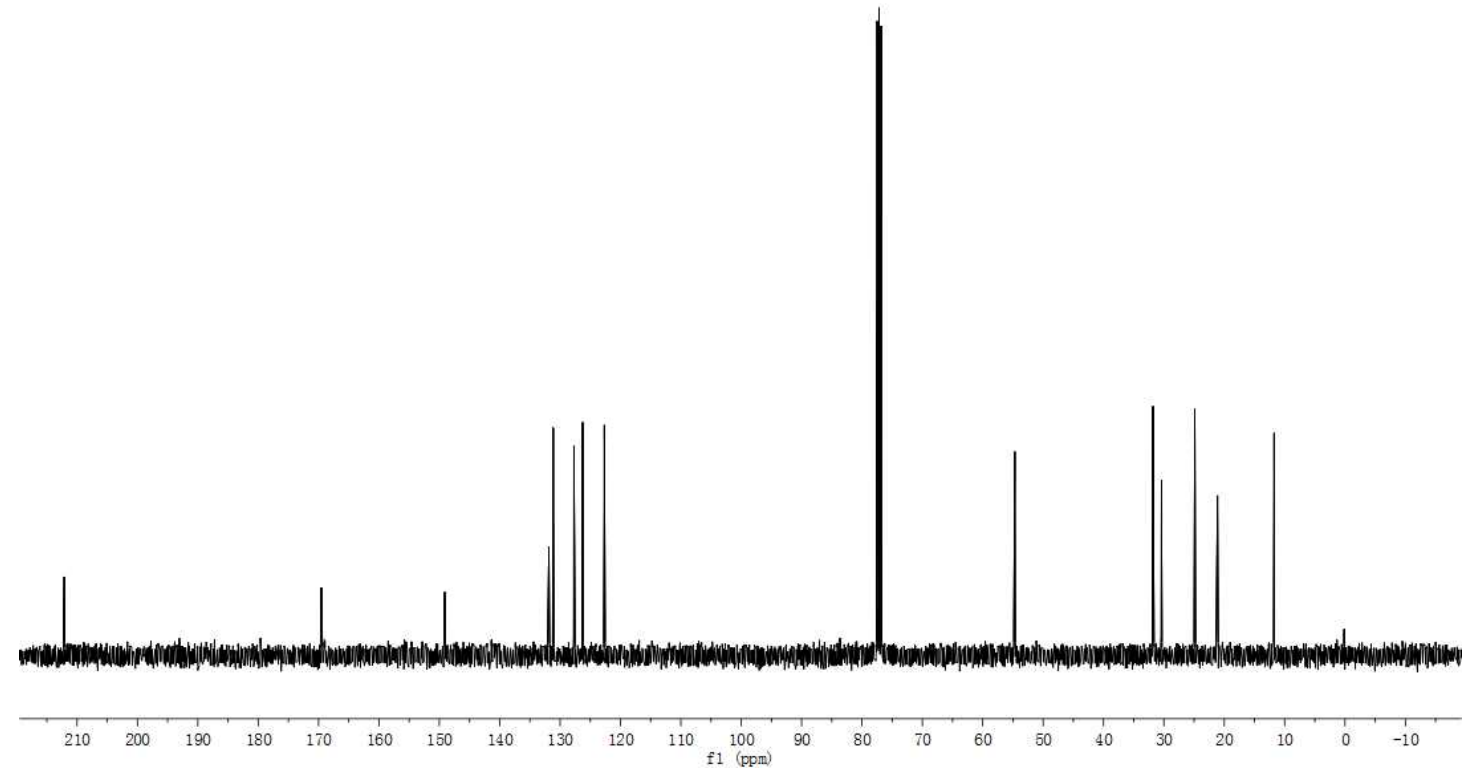




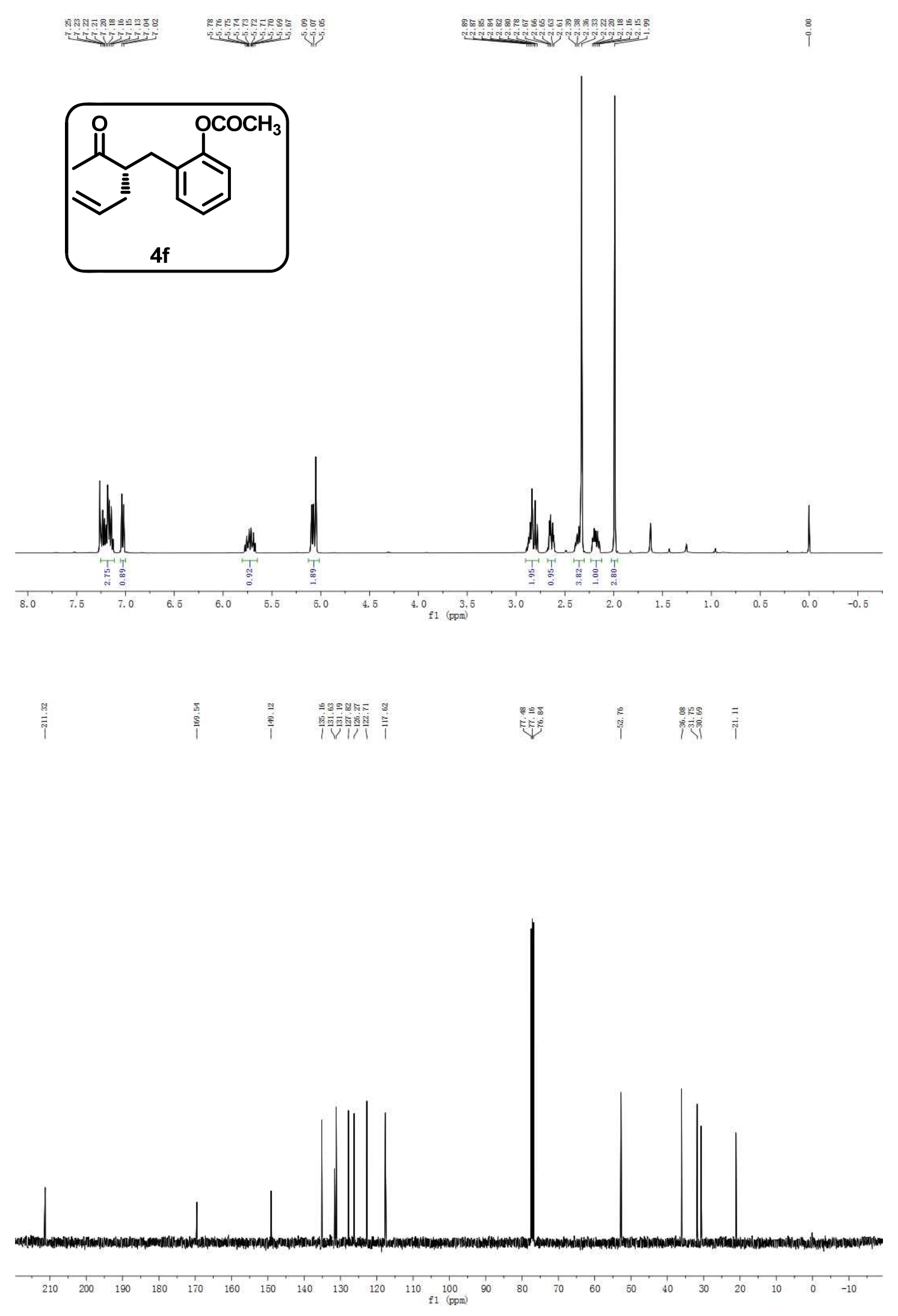



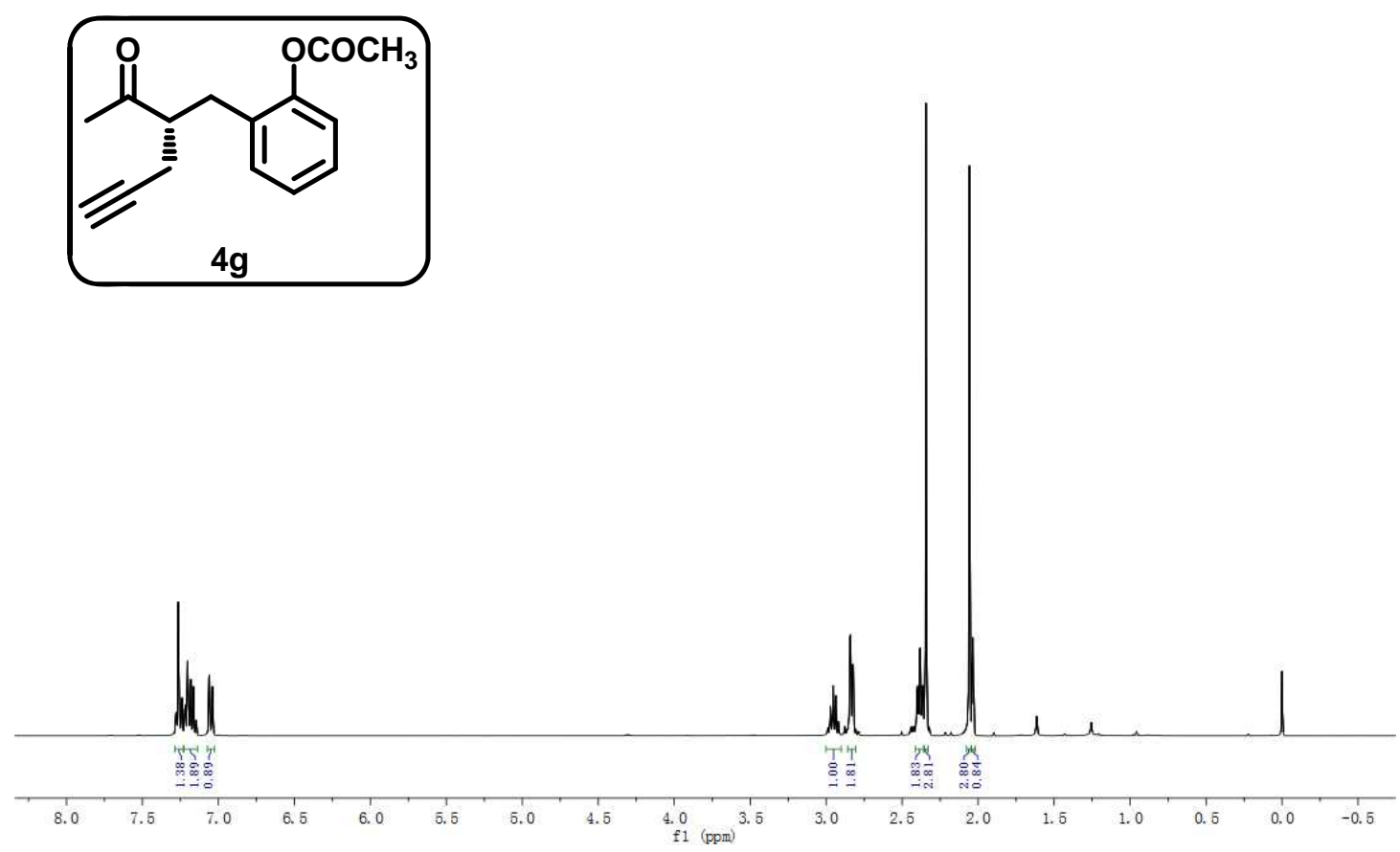

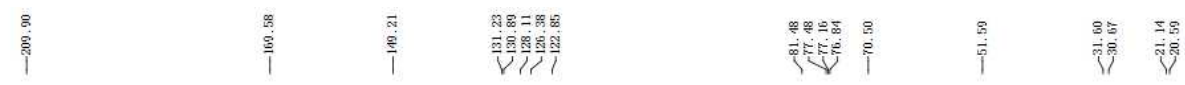

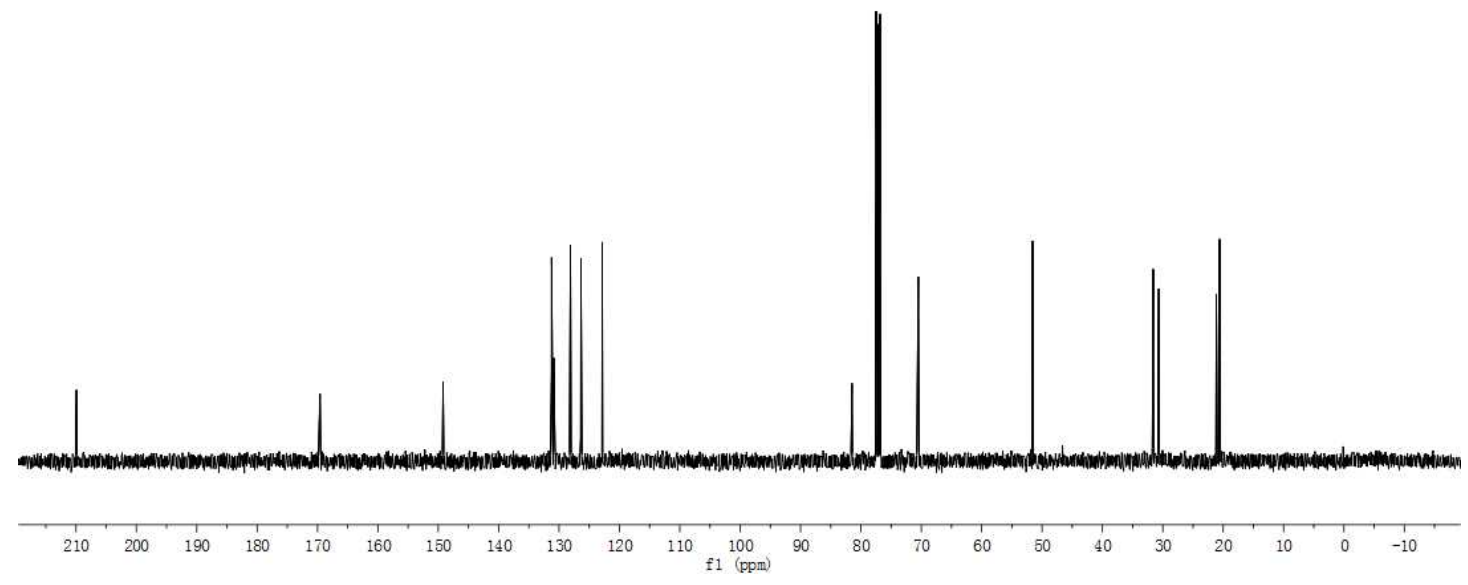



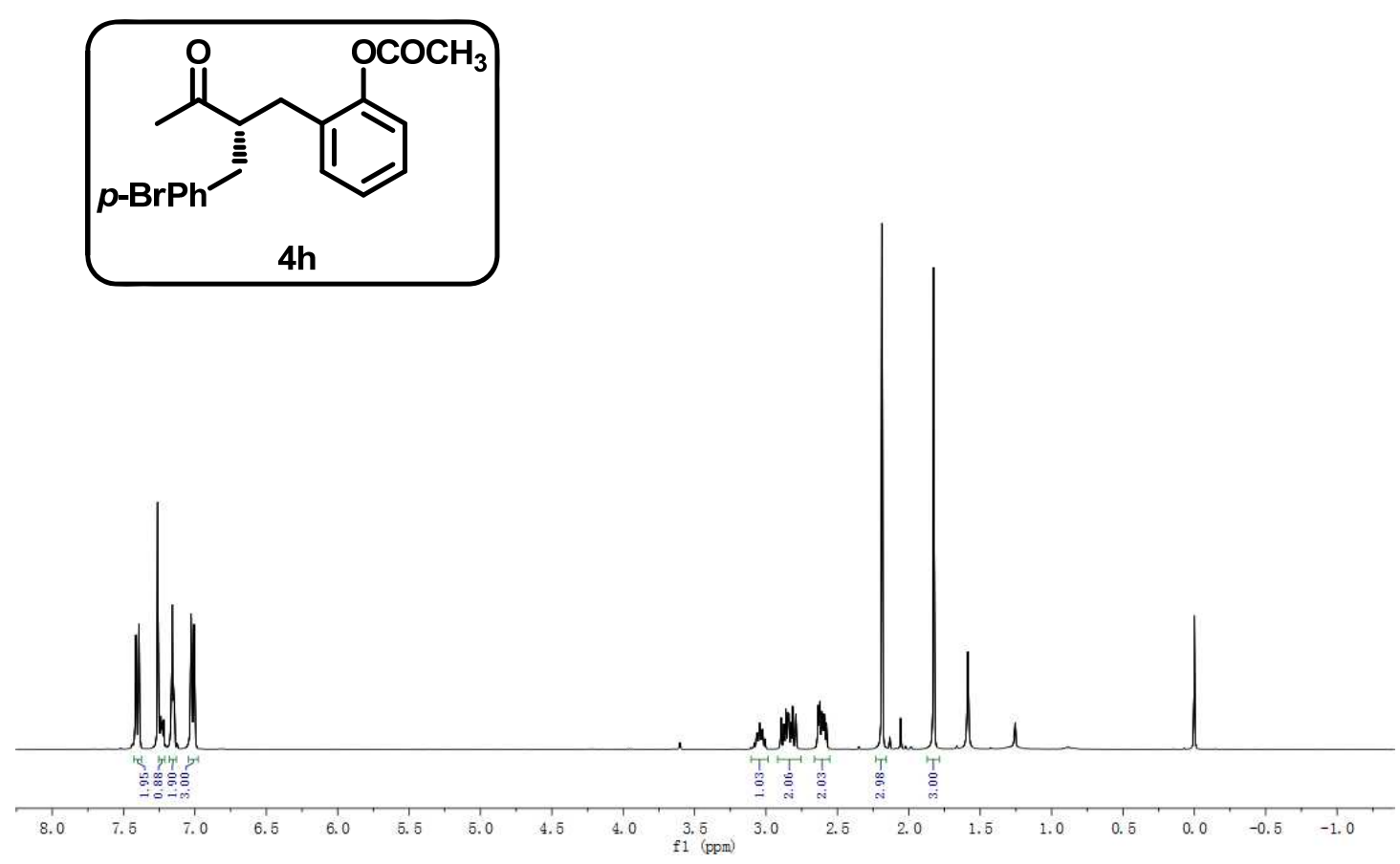

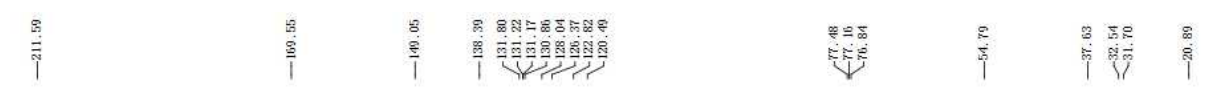

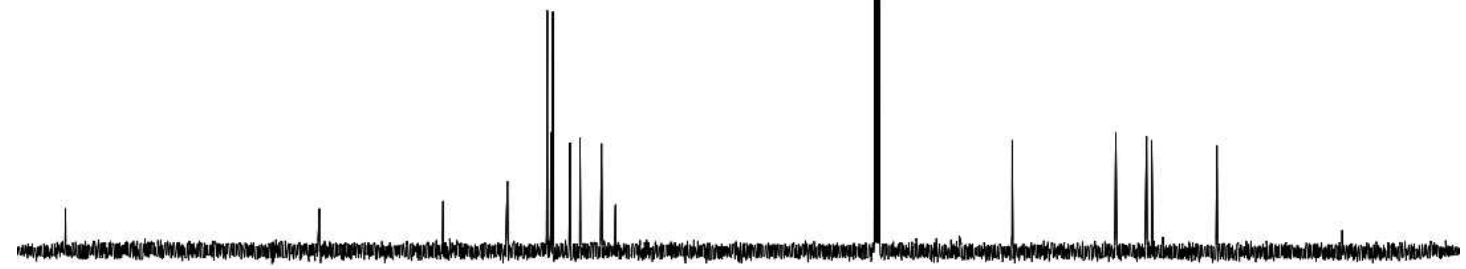

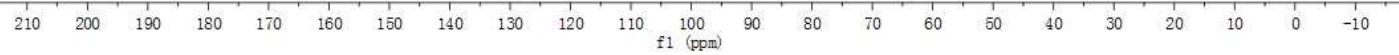



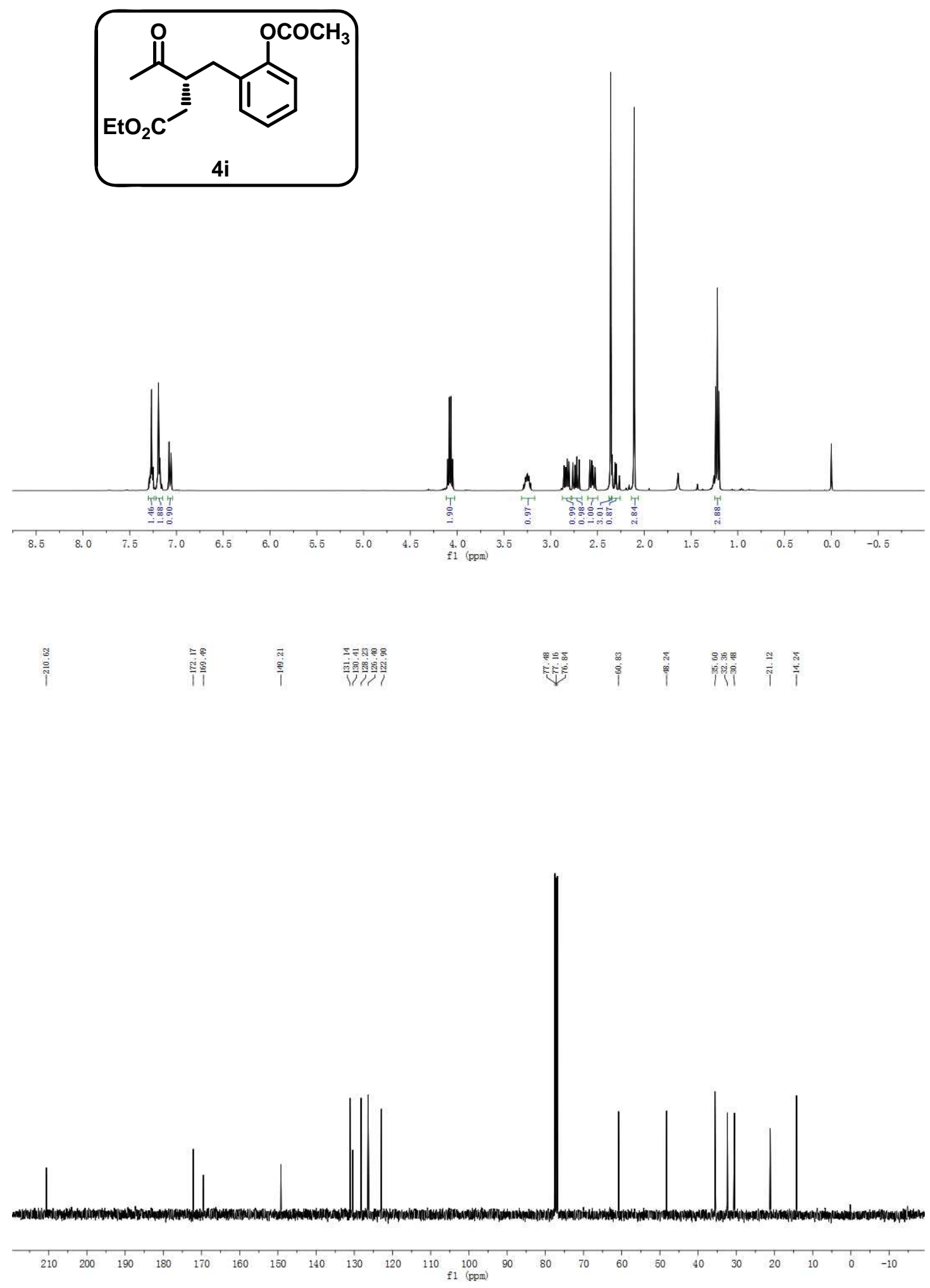

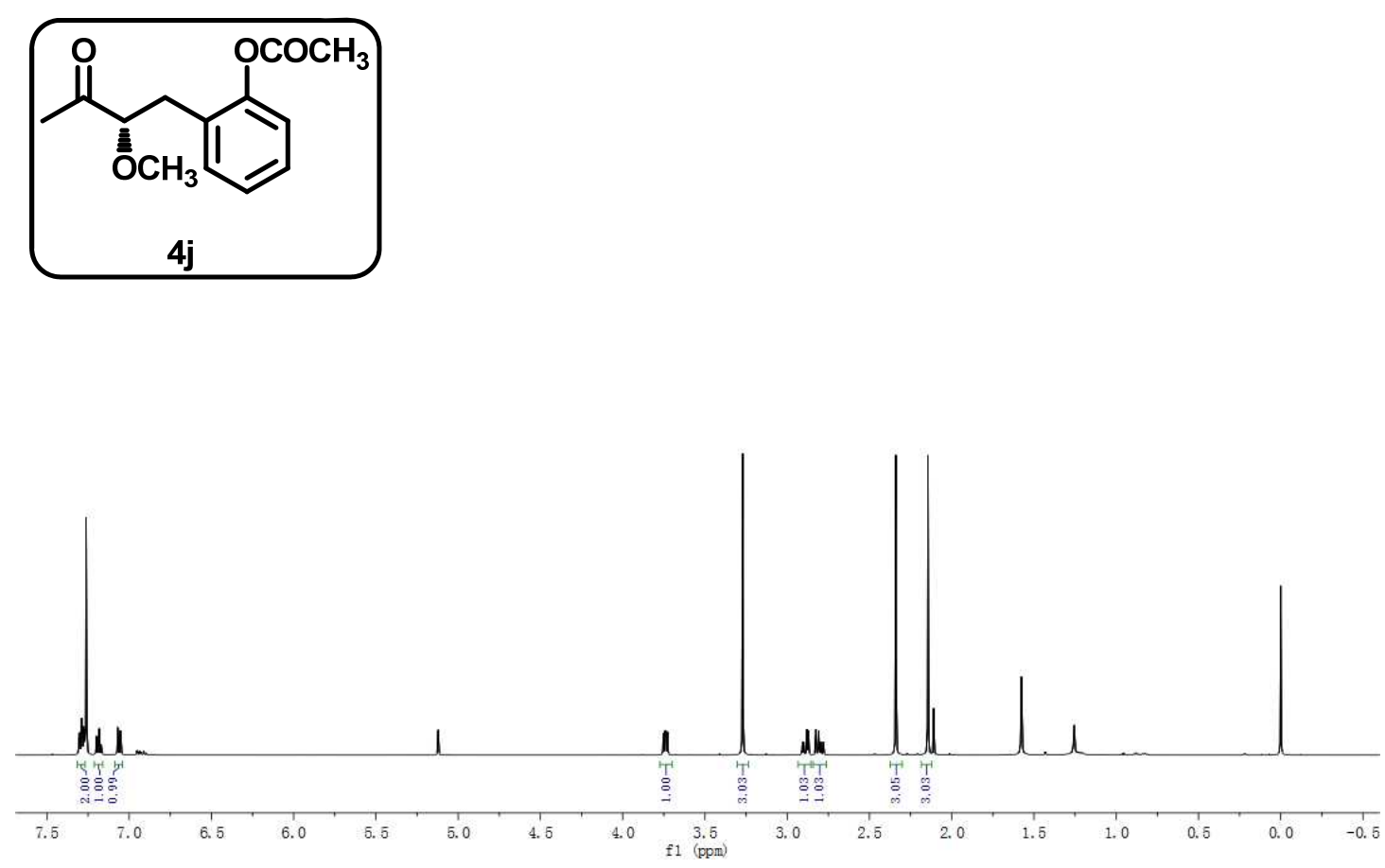

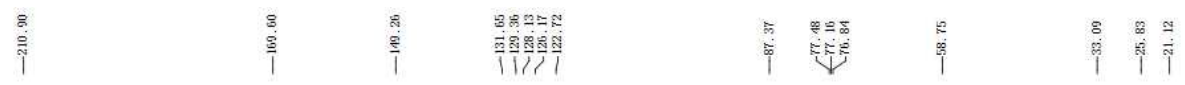

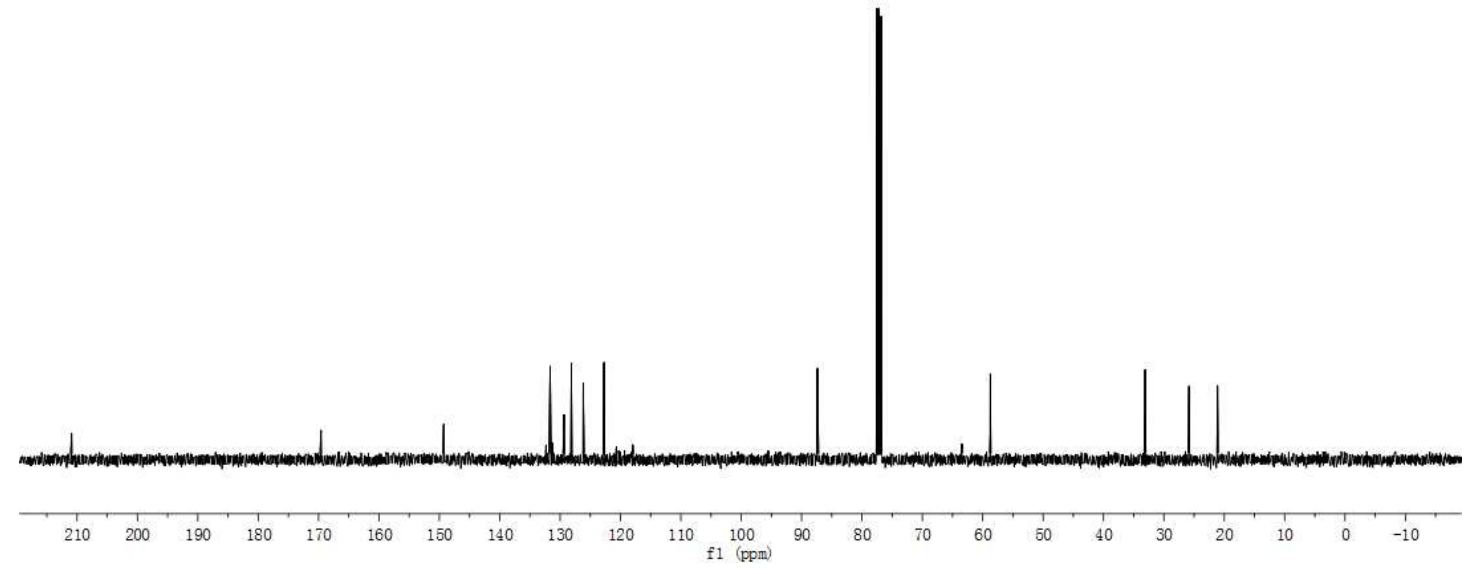



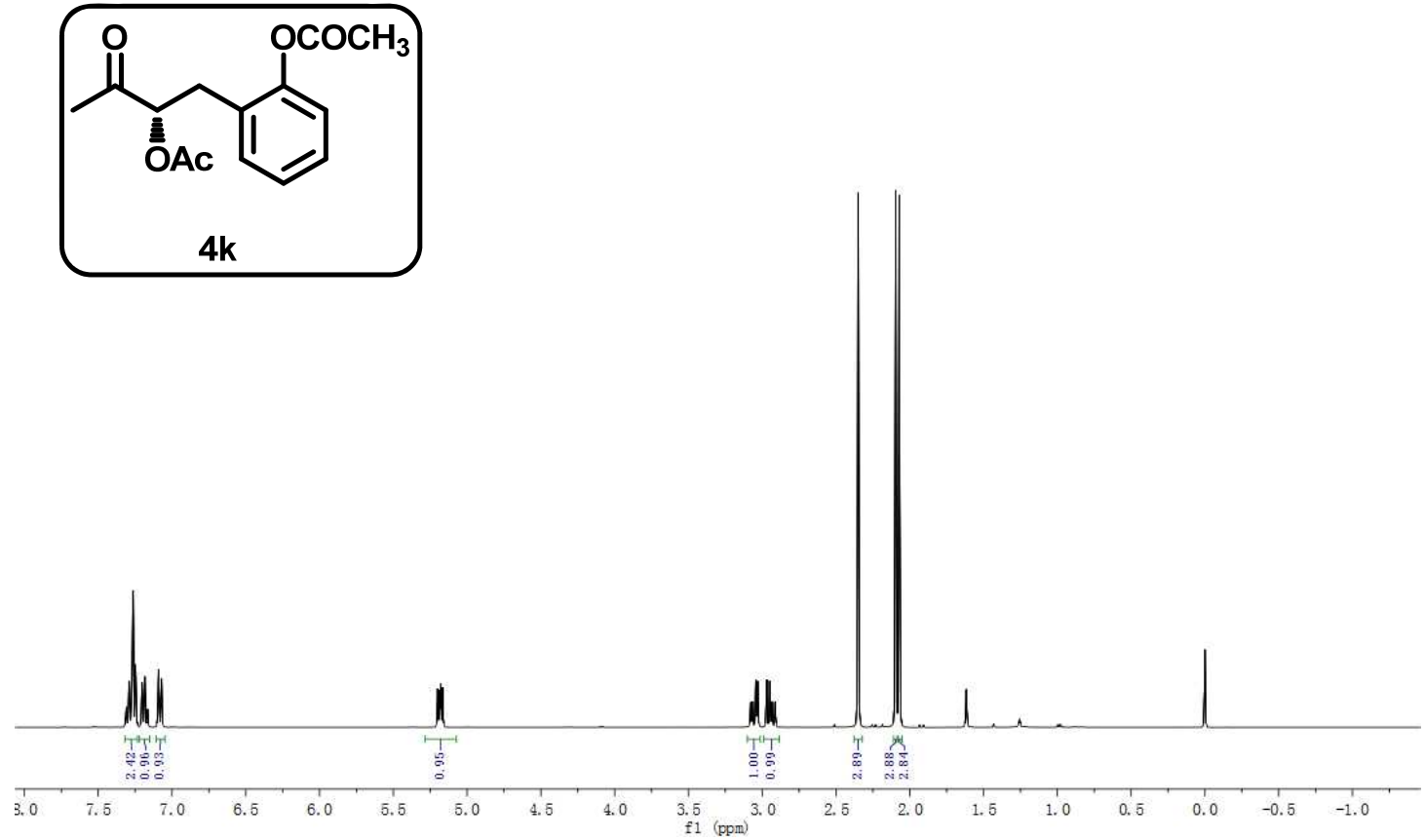

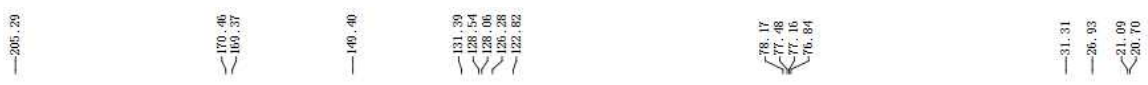

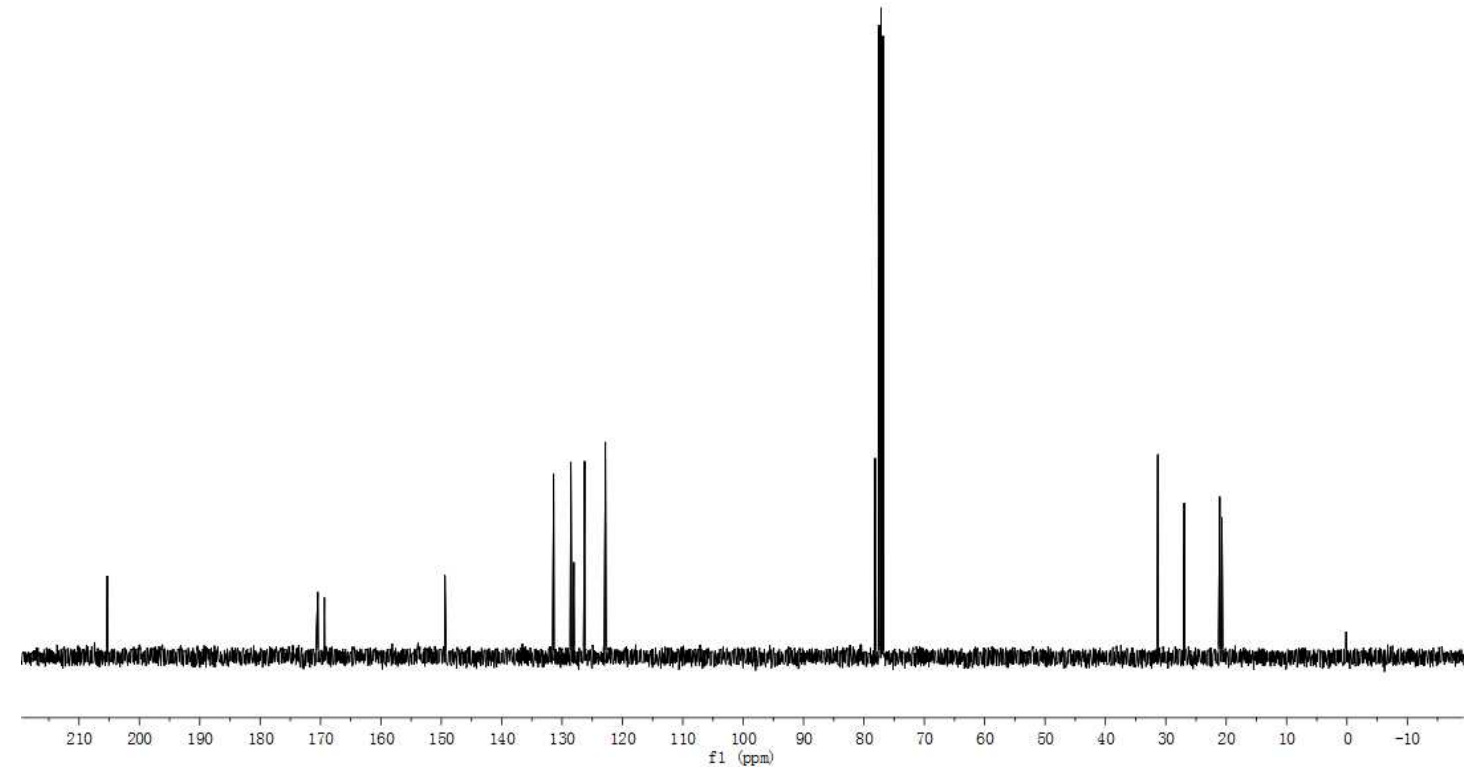




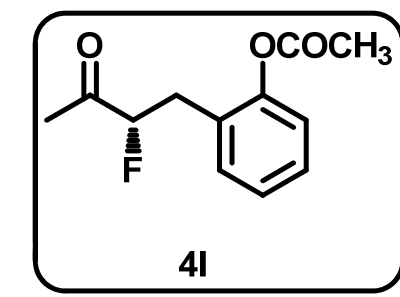

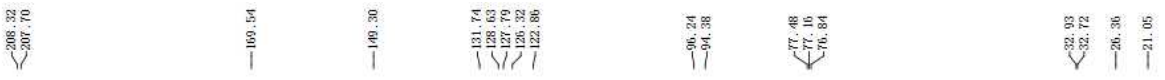
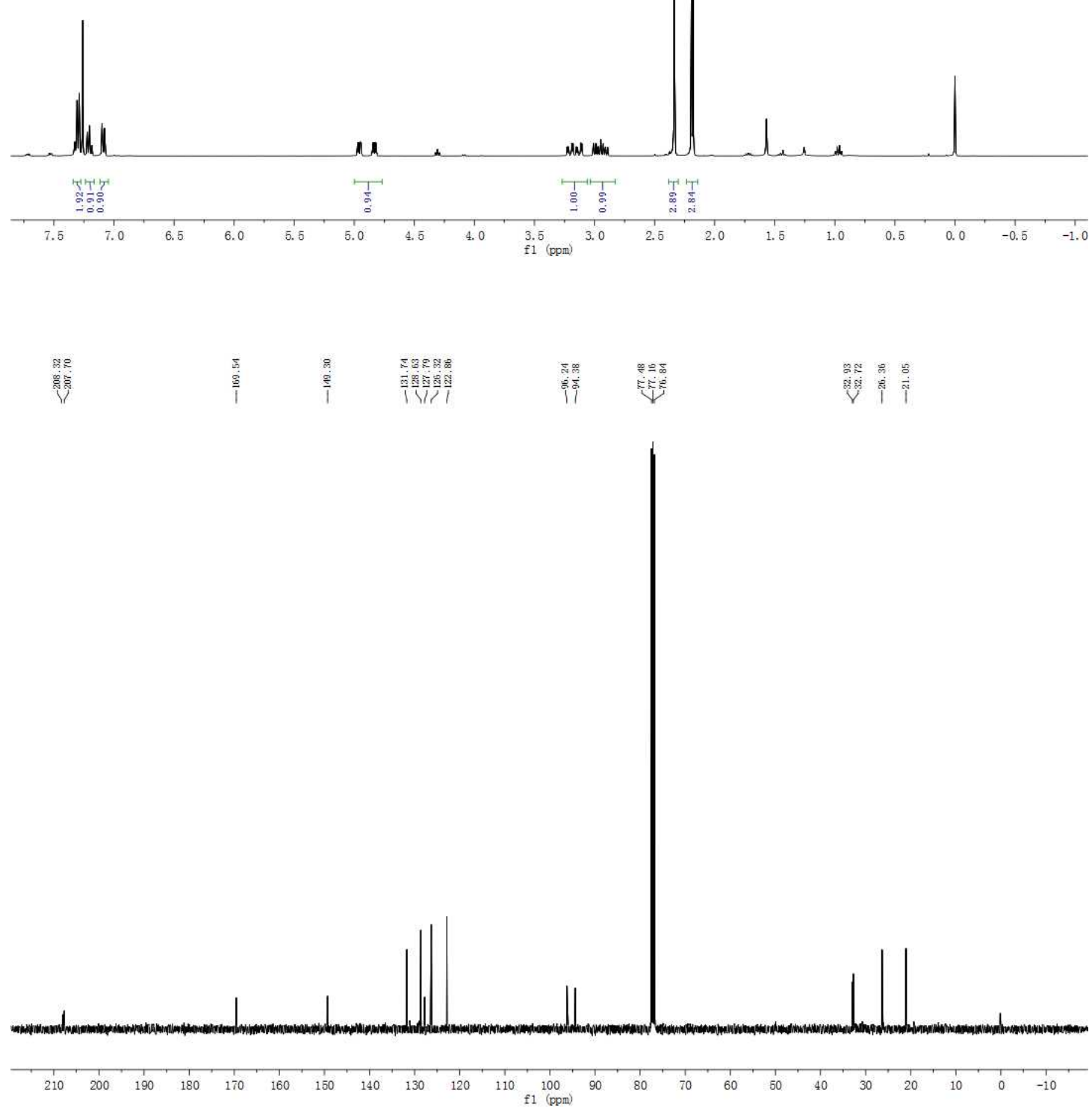

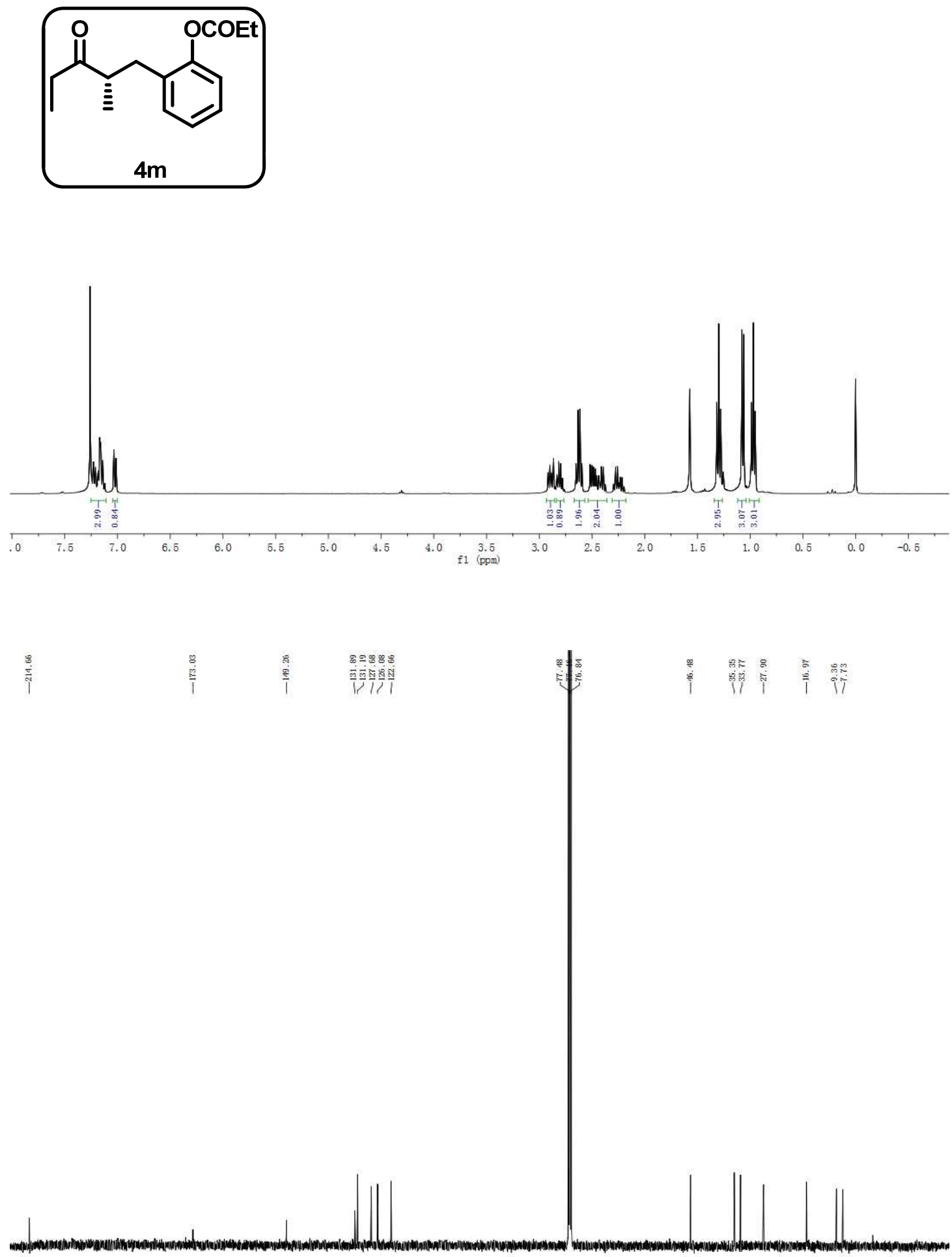

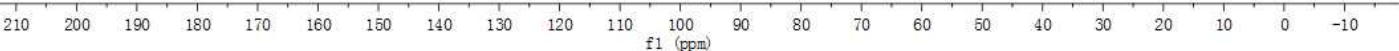



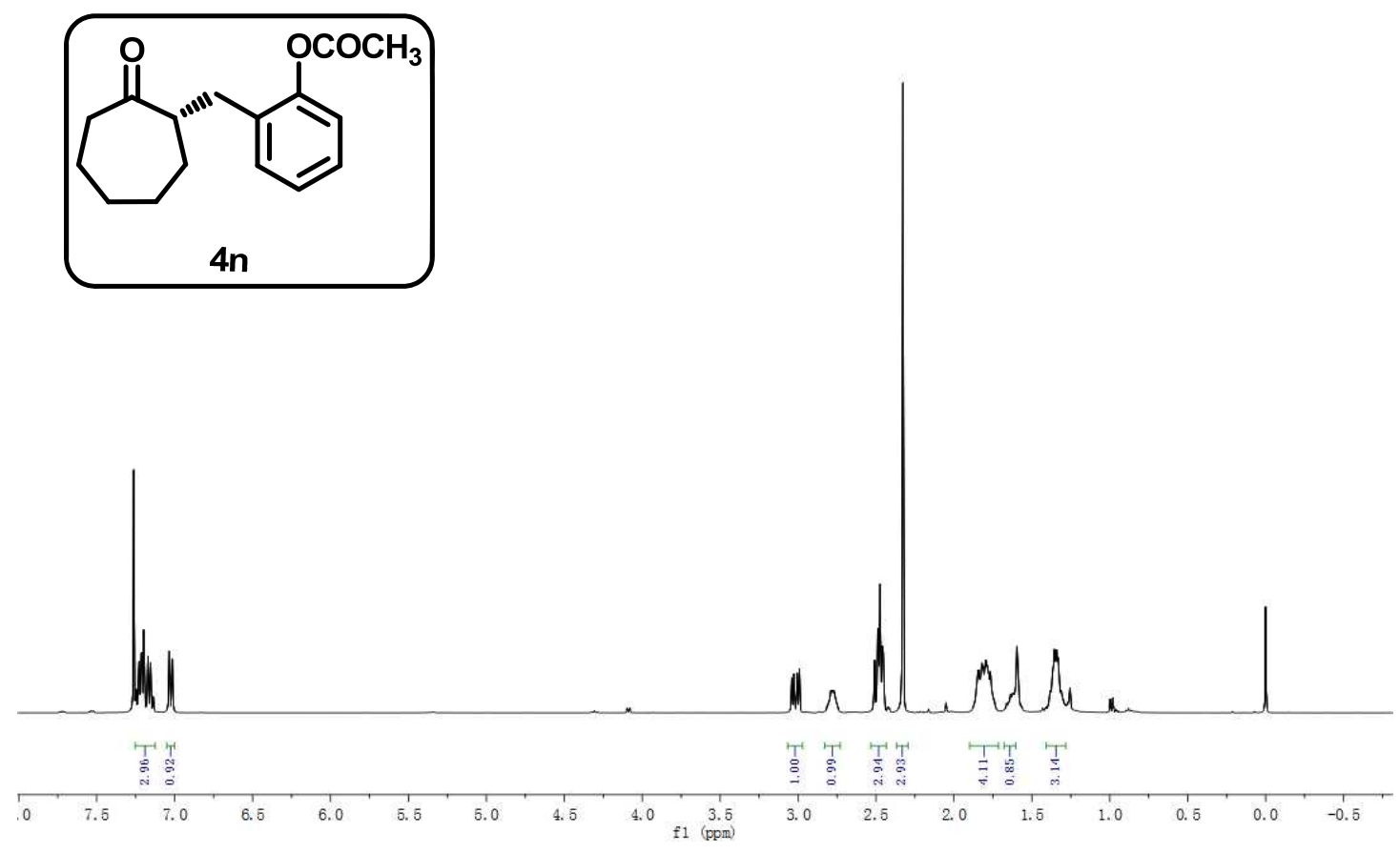

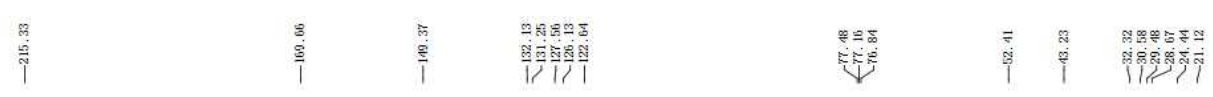

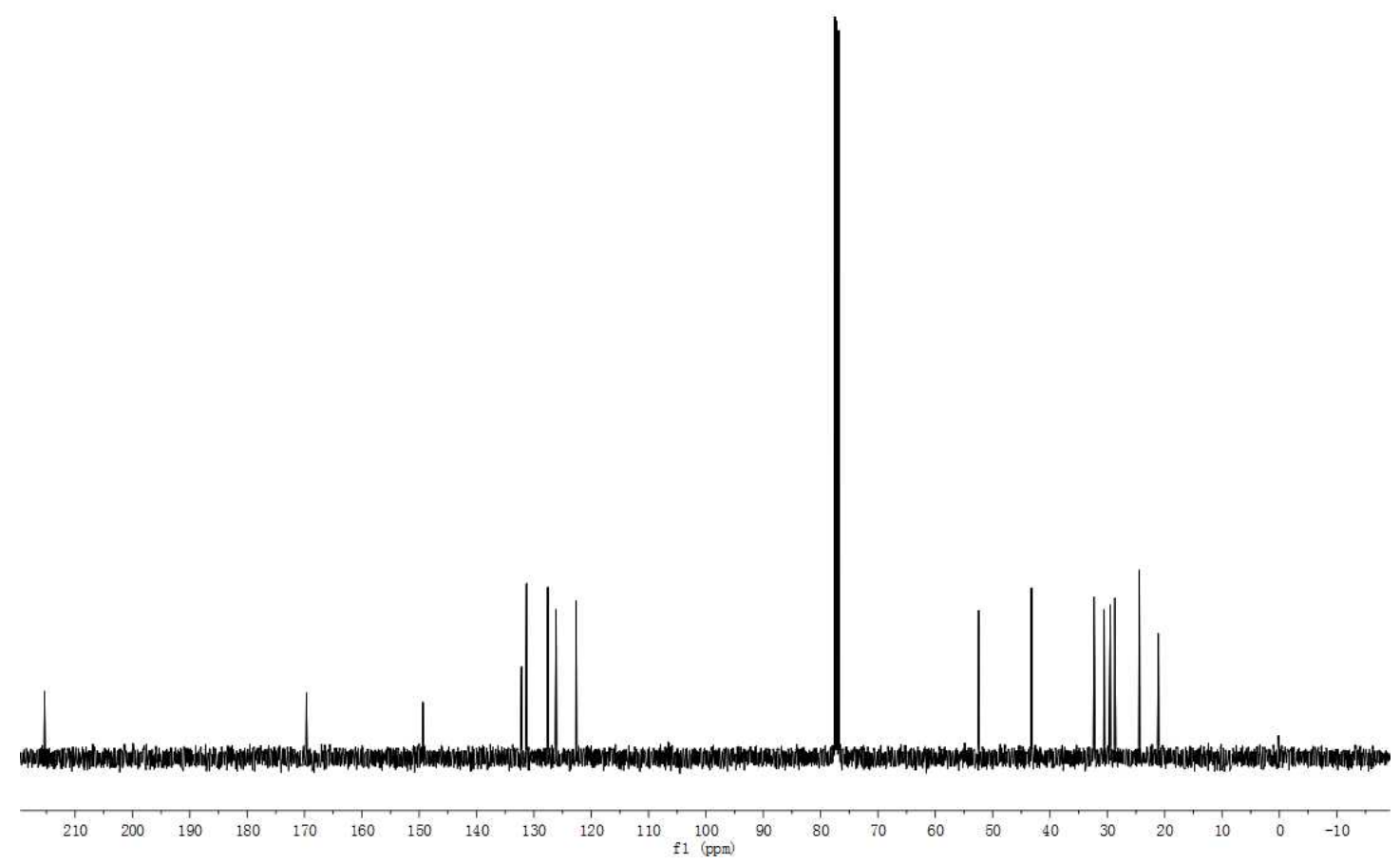



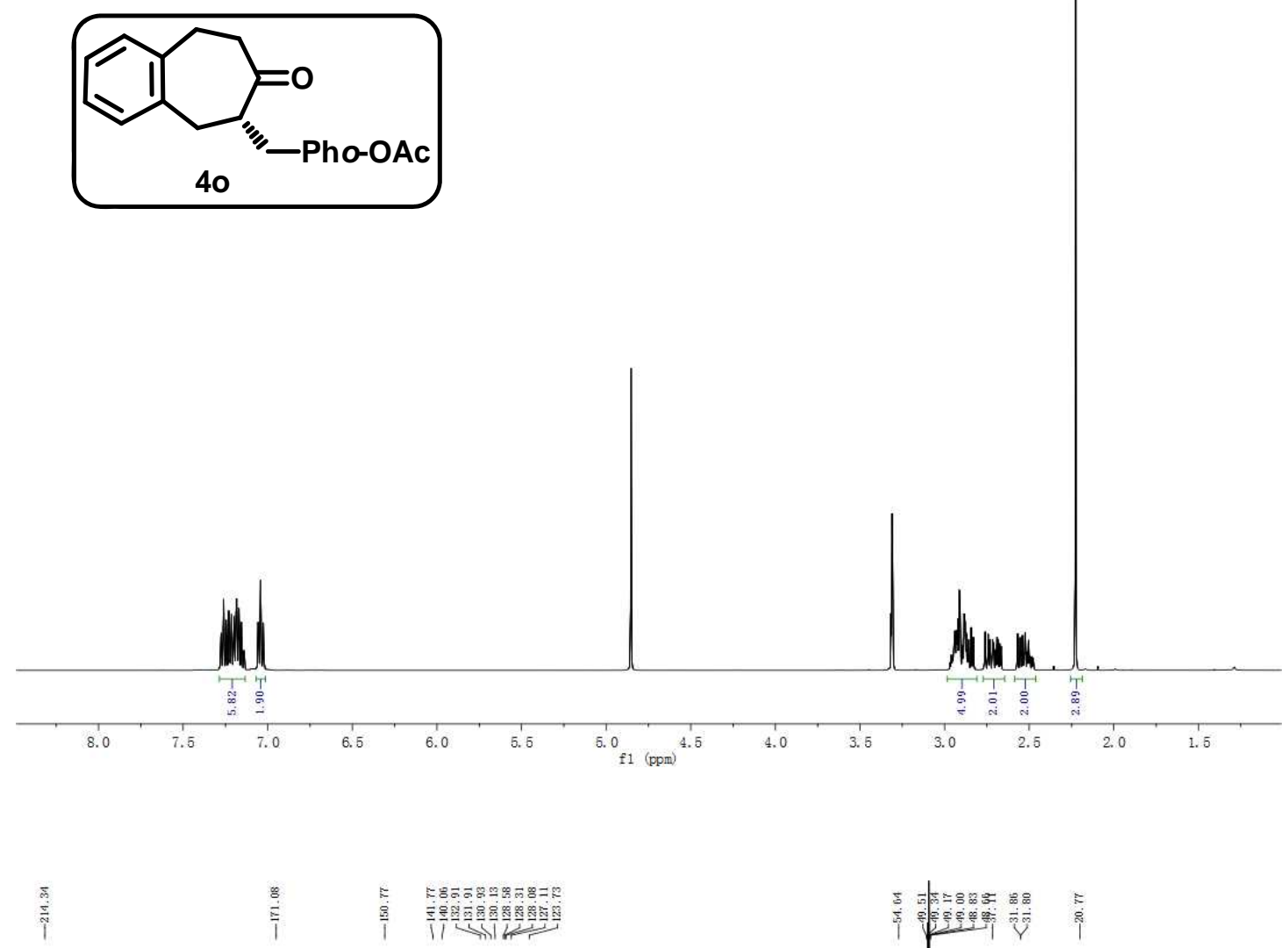

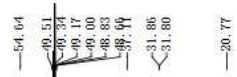

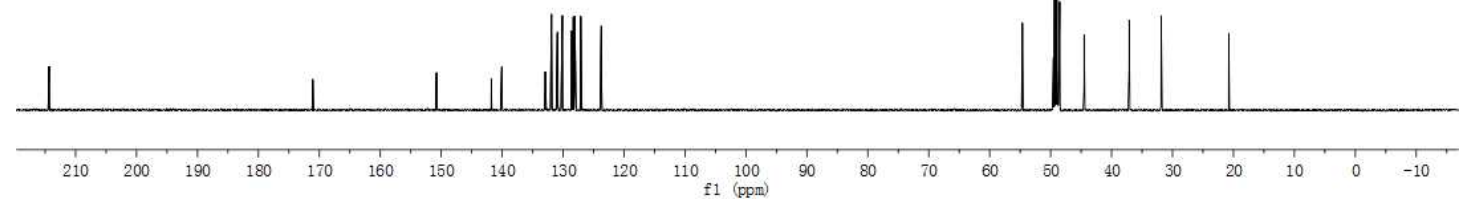




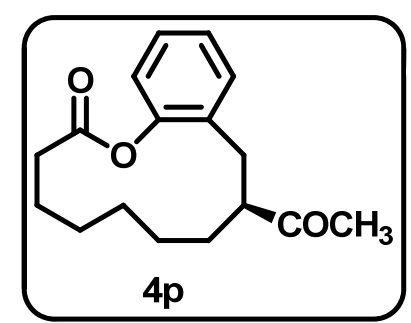

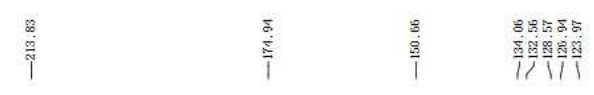
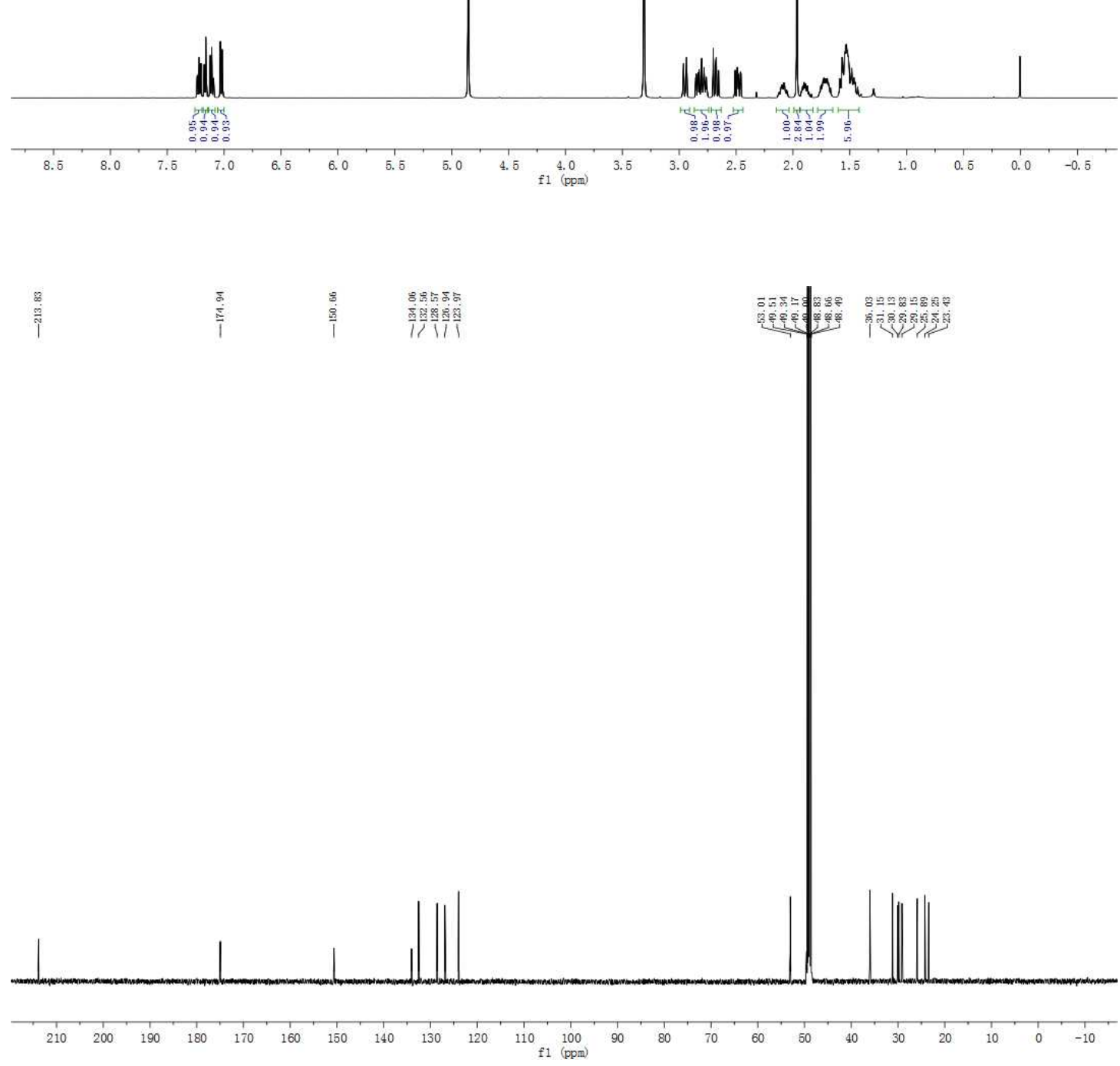

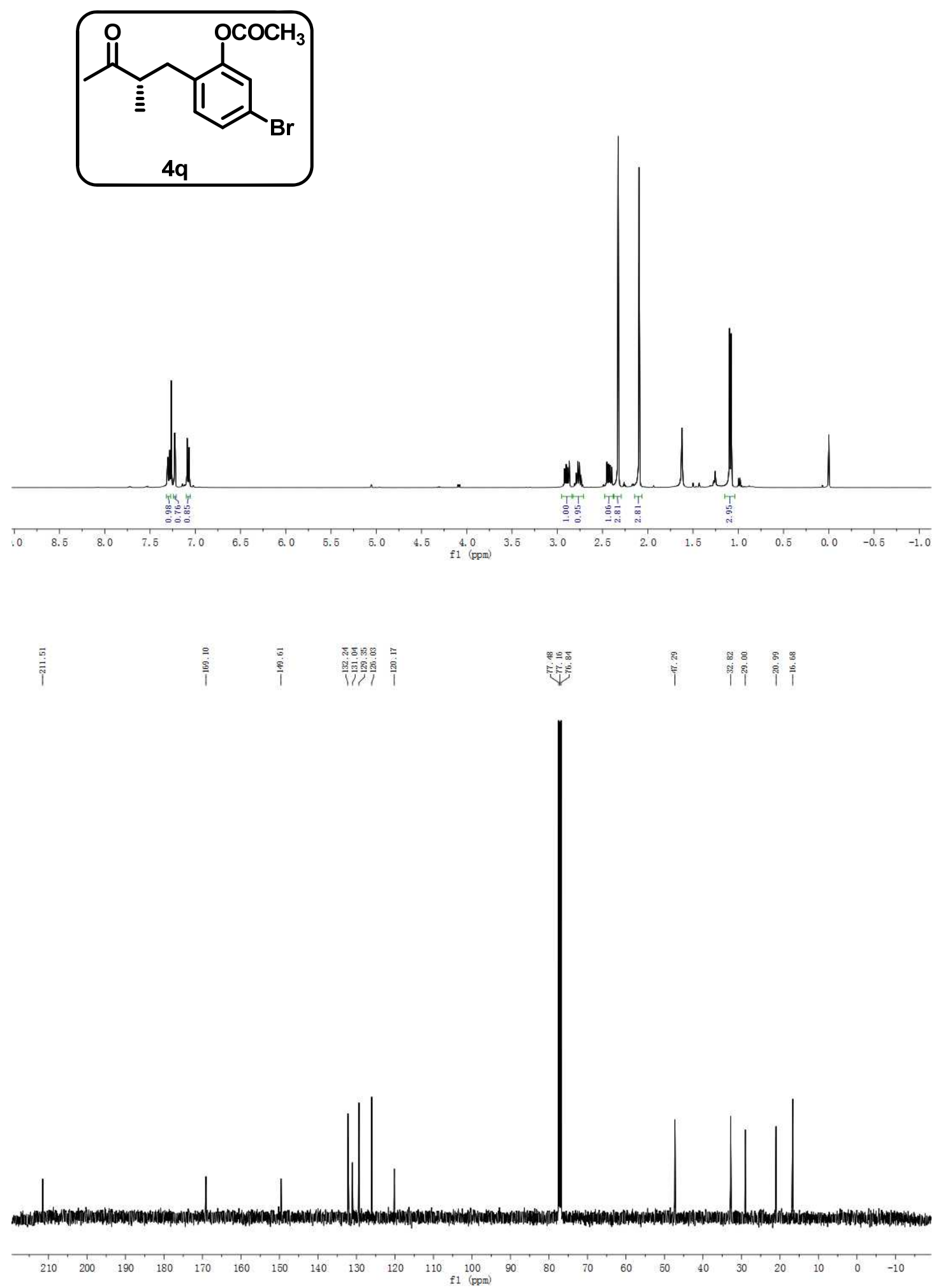

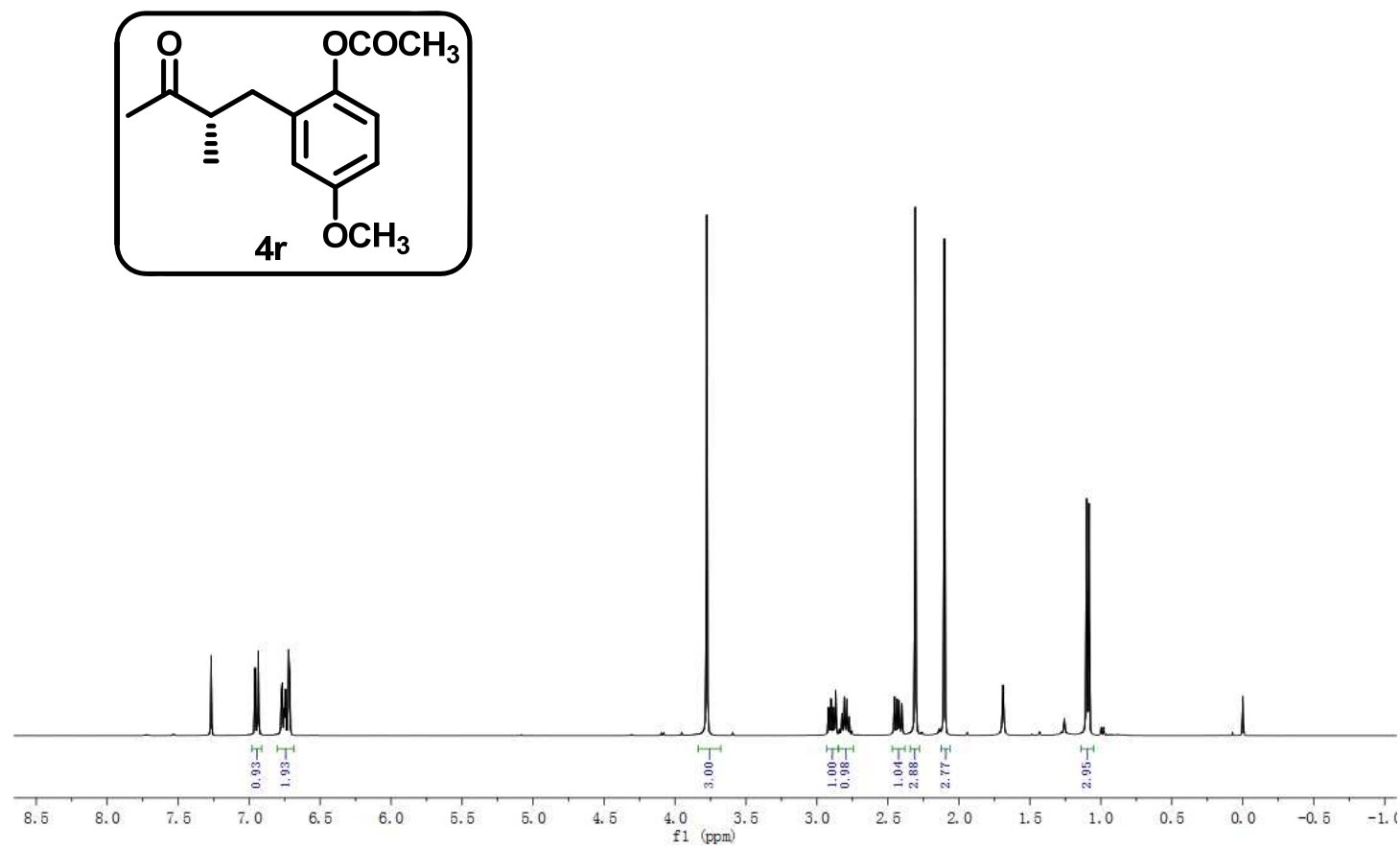

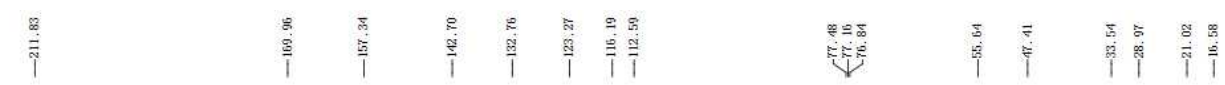

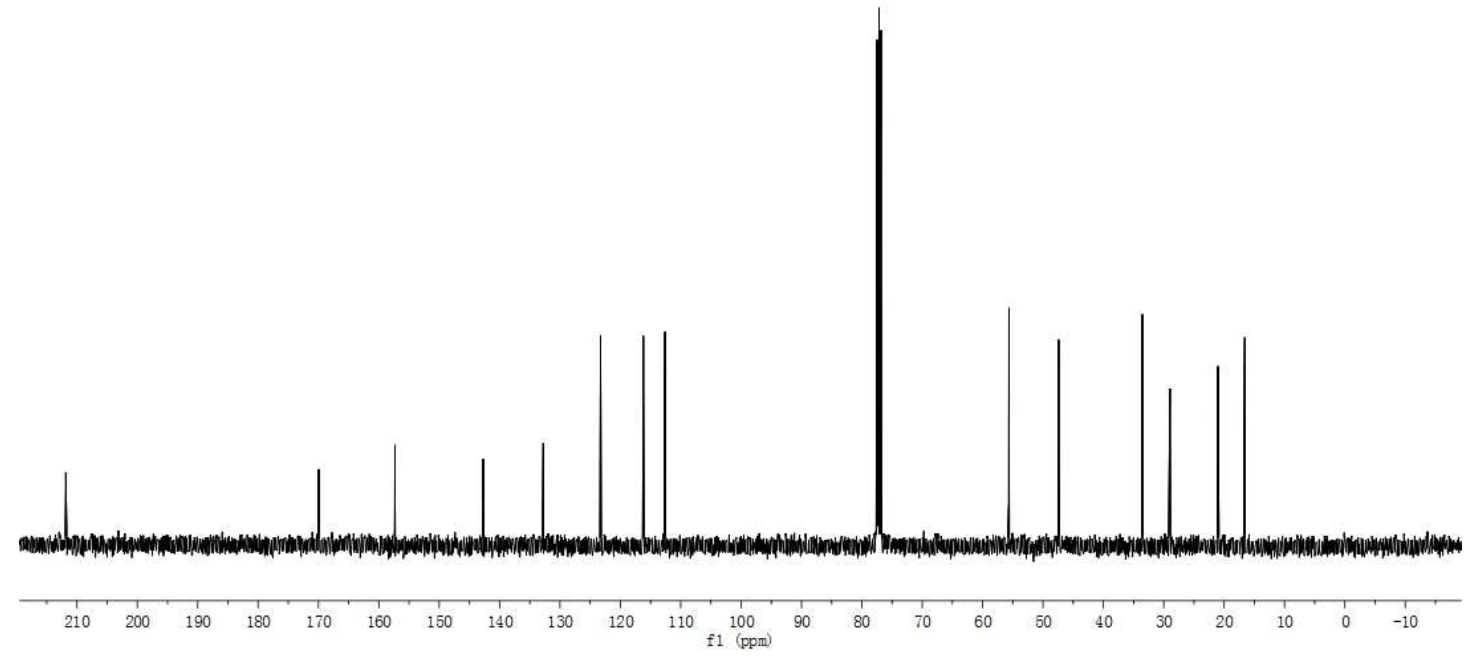



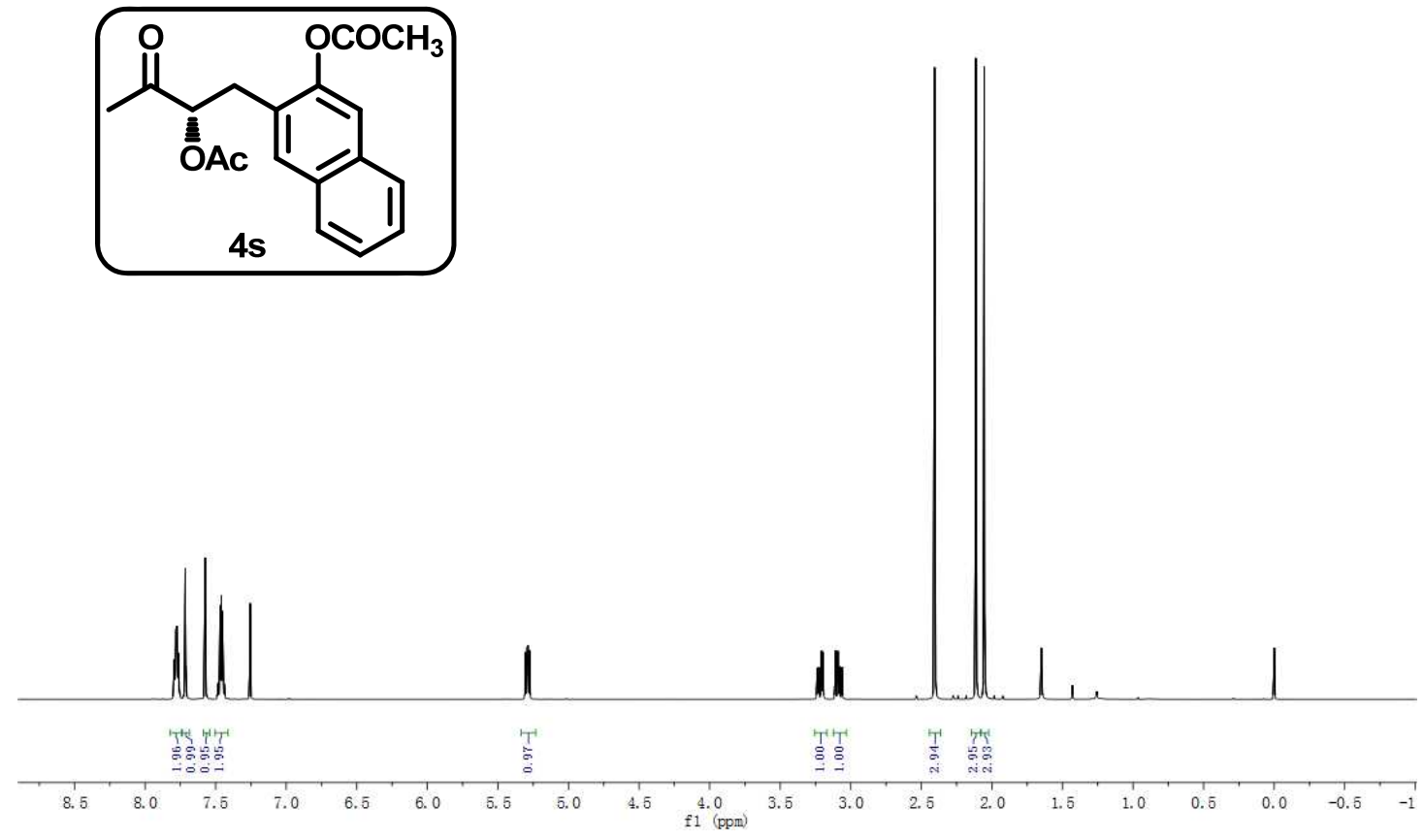

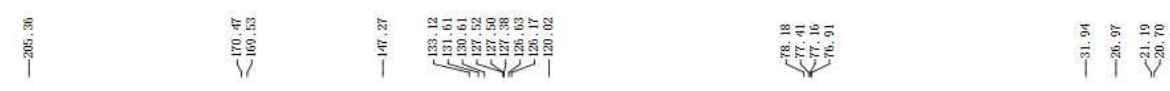

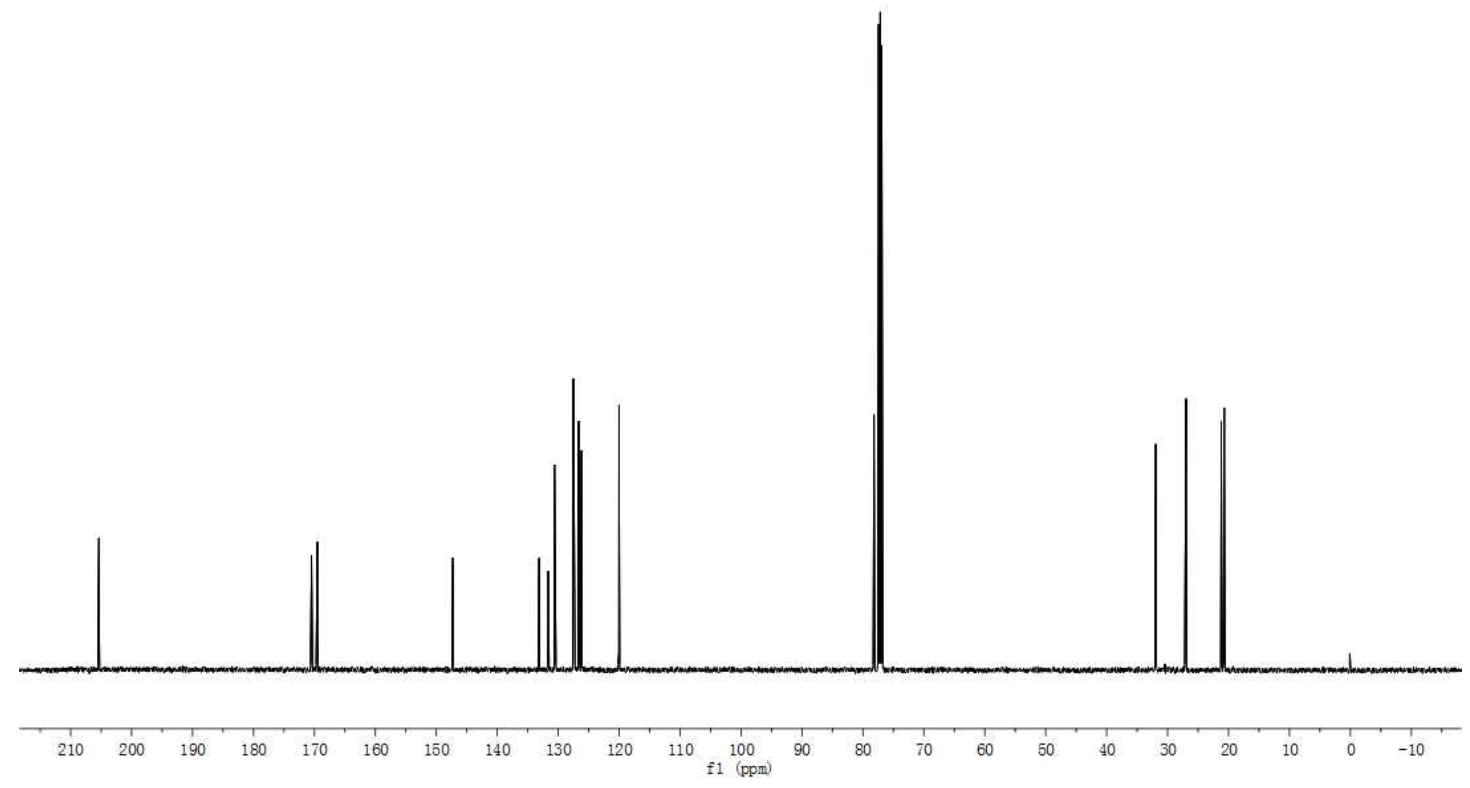




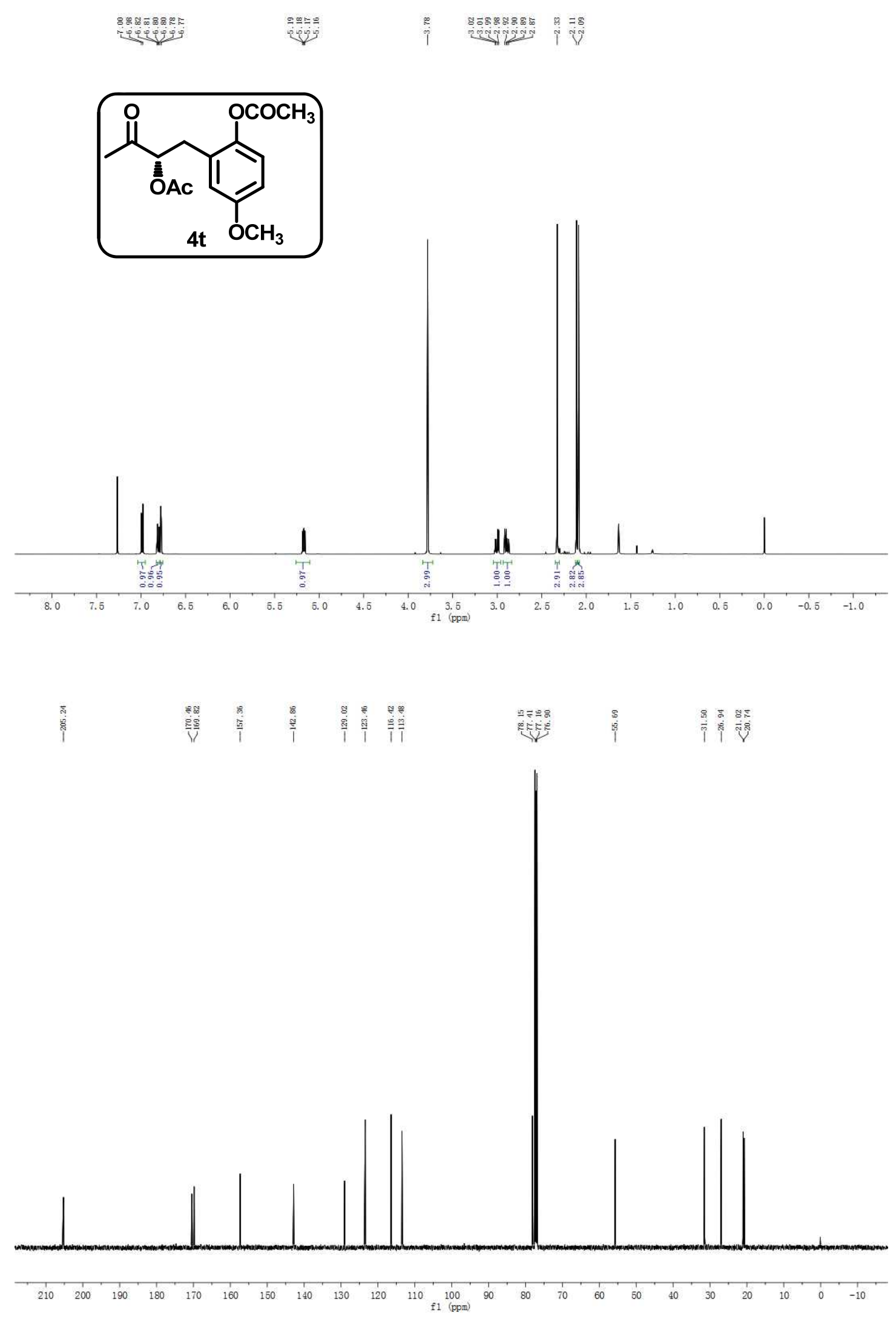




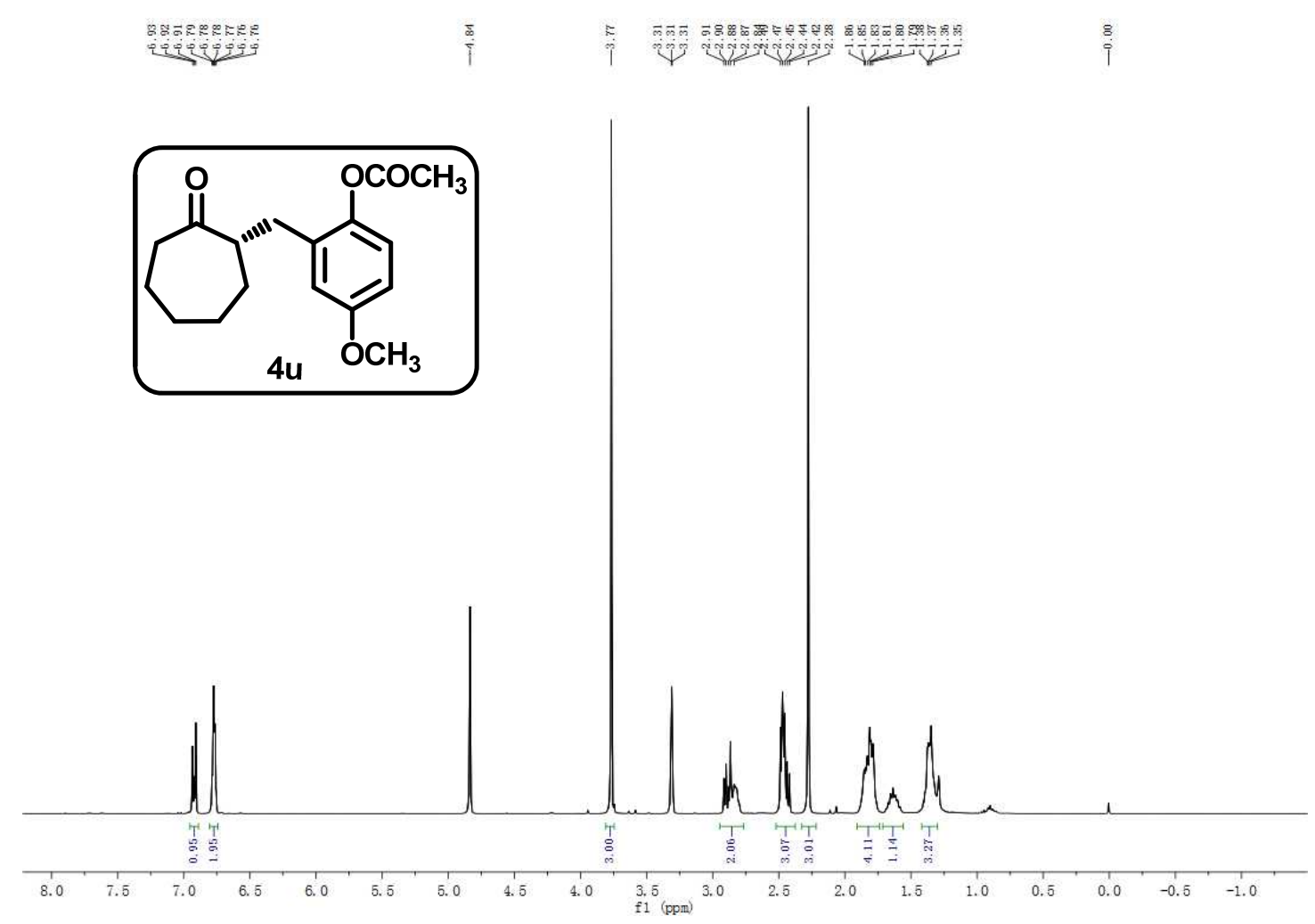

1 $1111 \%$

gas:

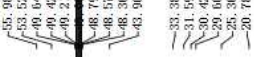

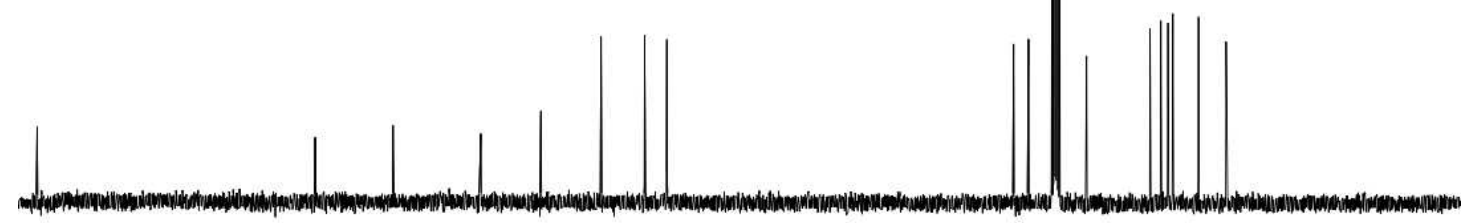

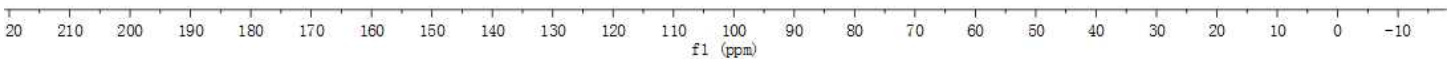




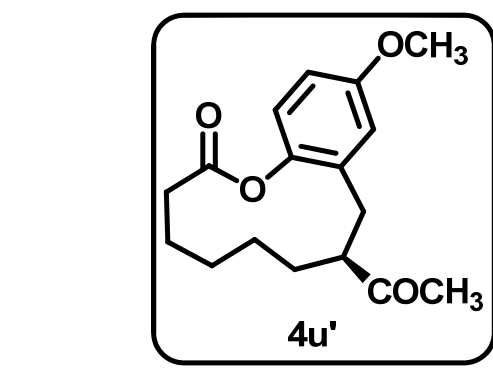

14111

\section{ำร}

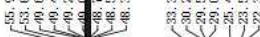

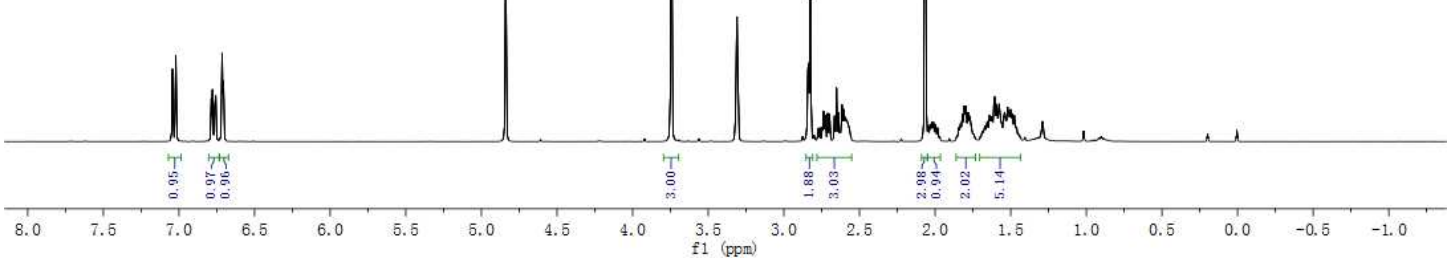



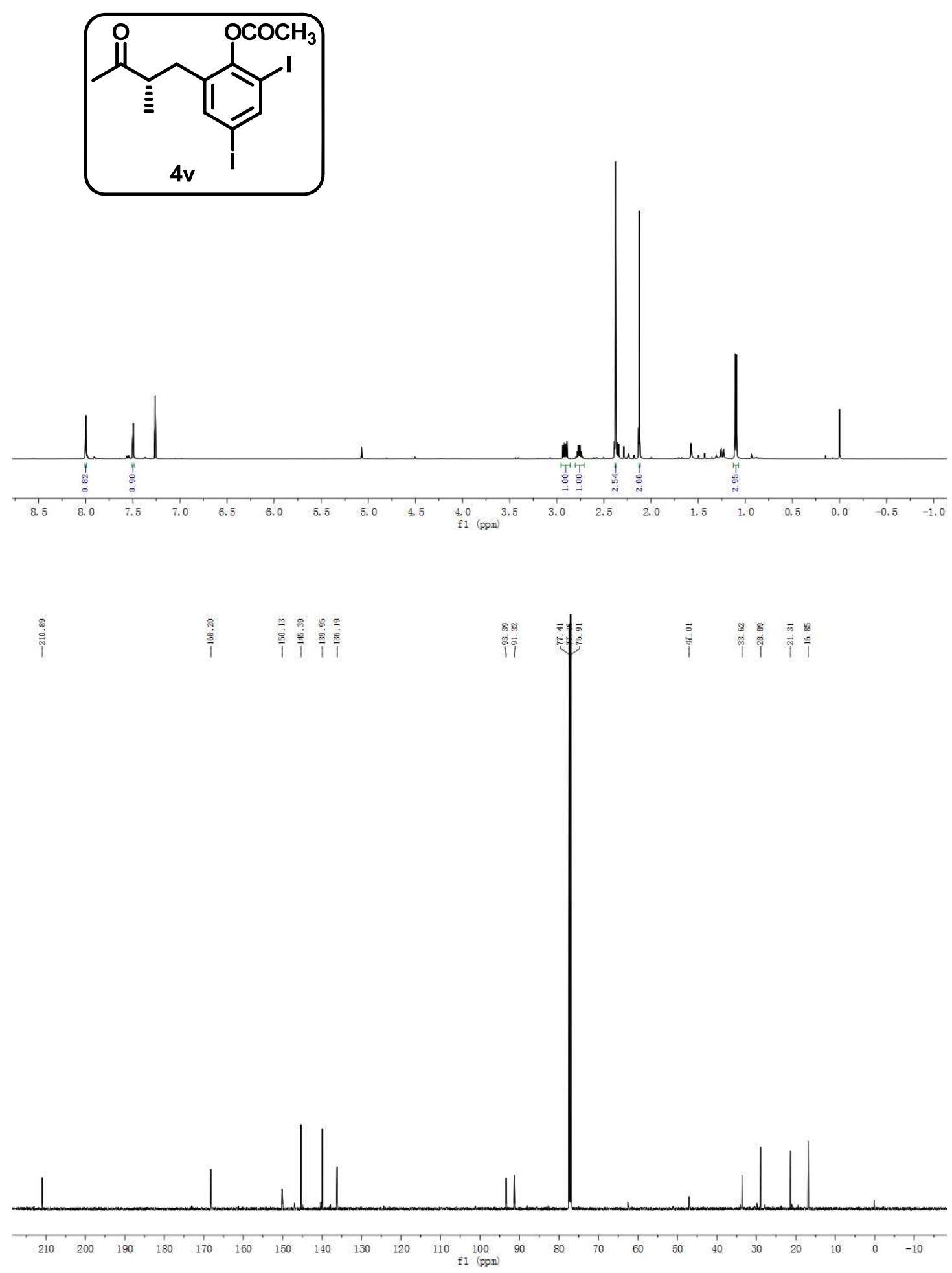

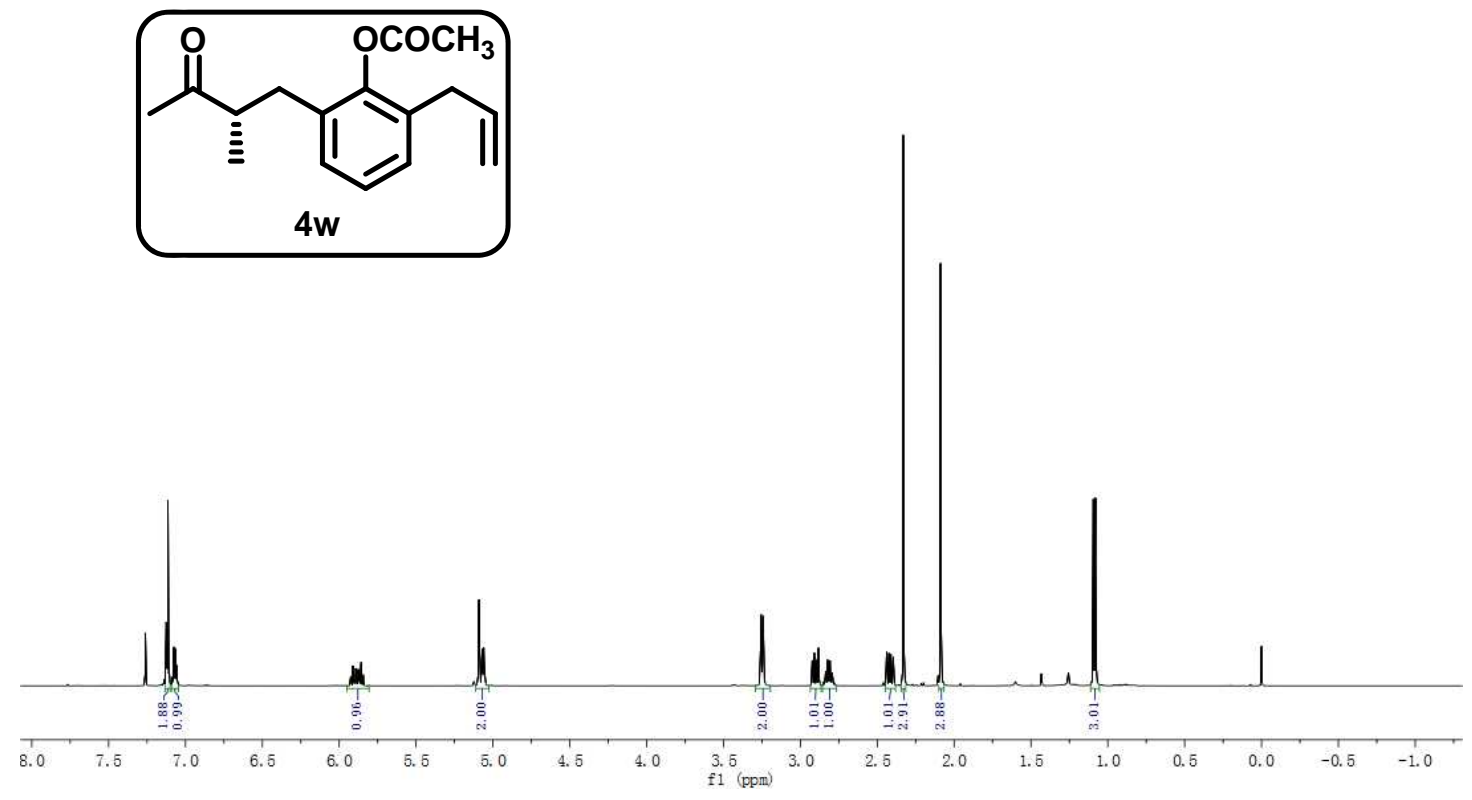

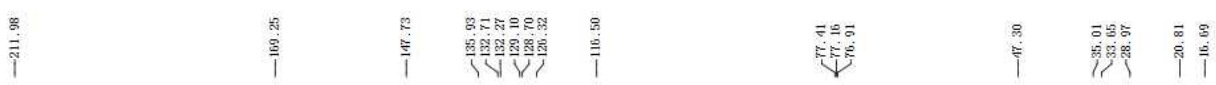

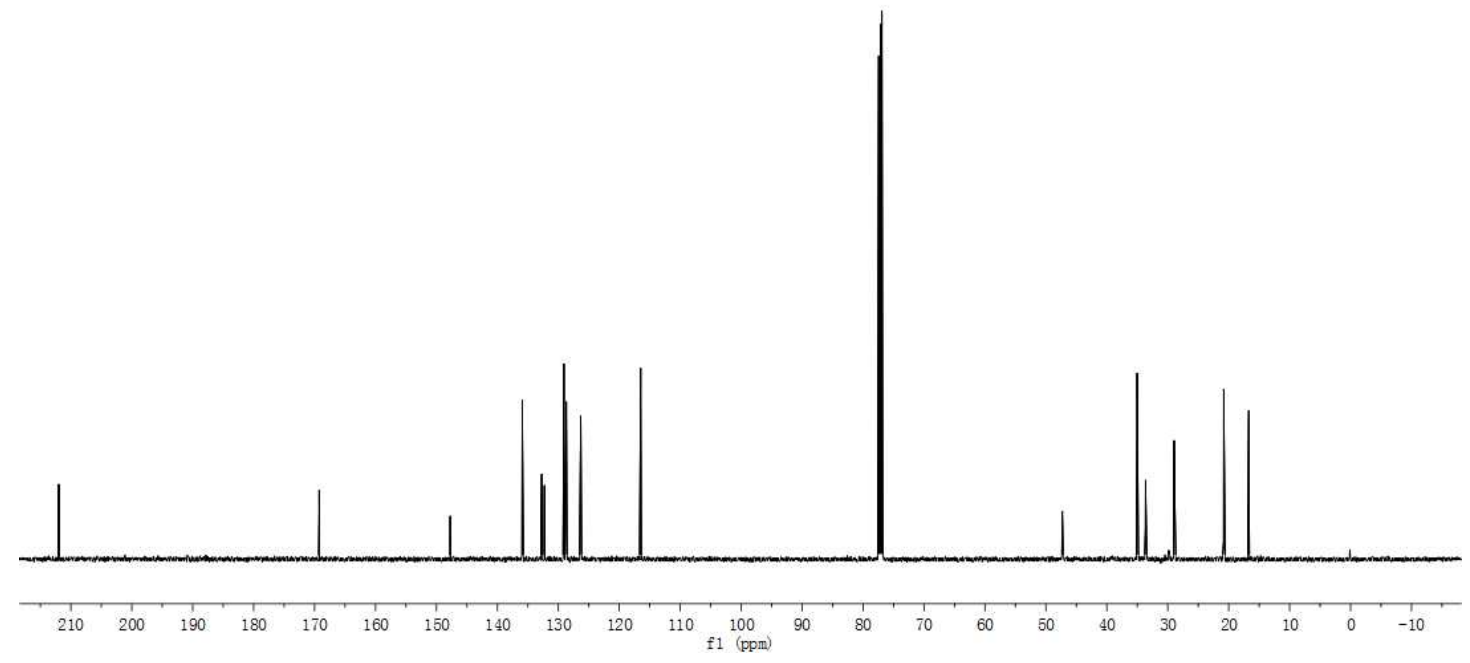



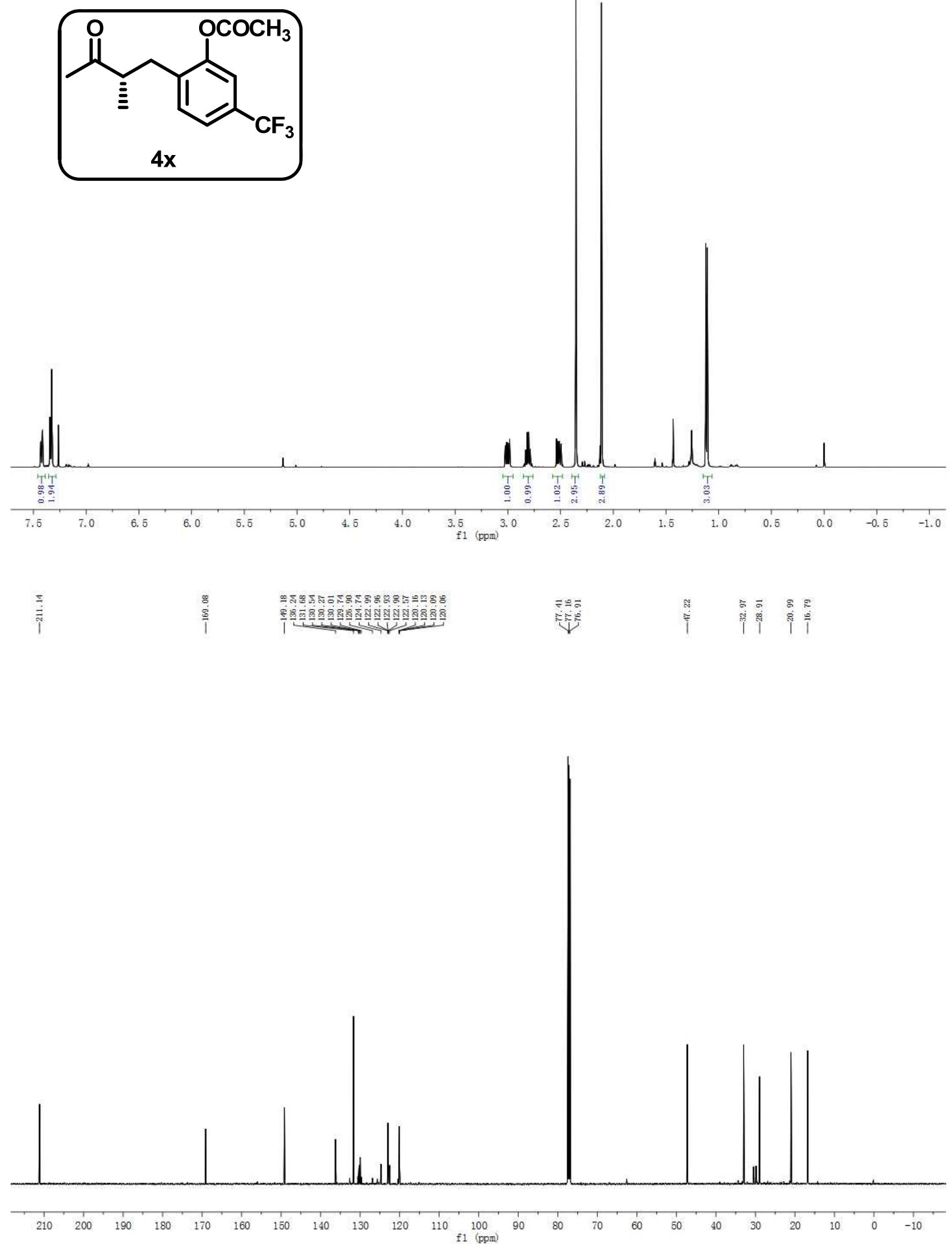

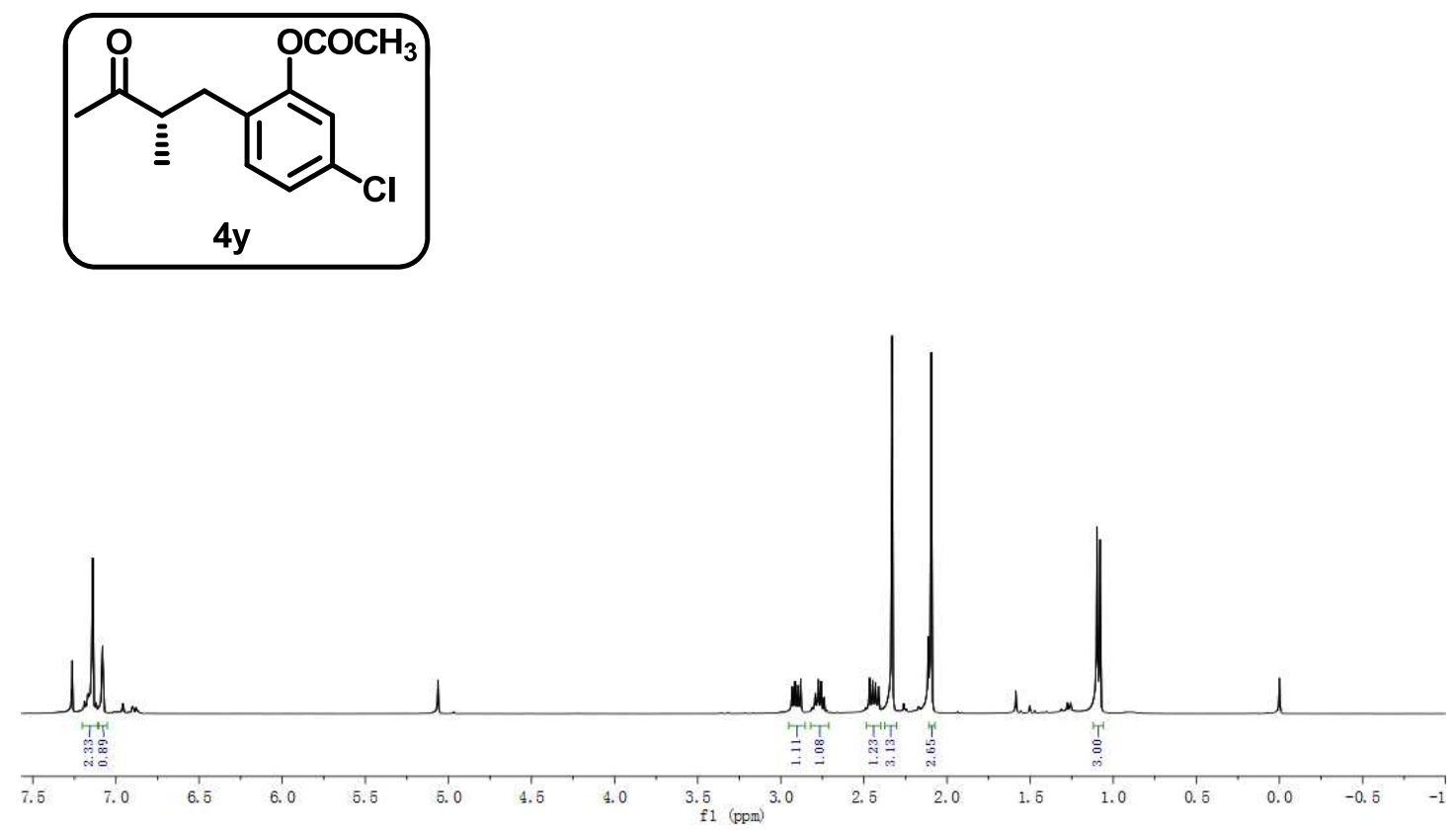

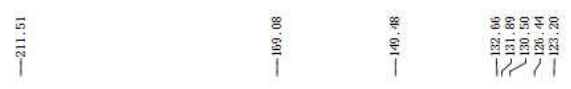

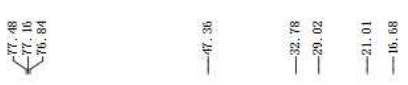

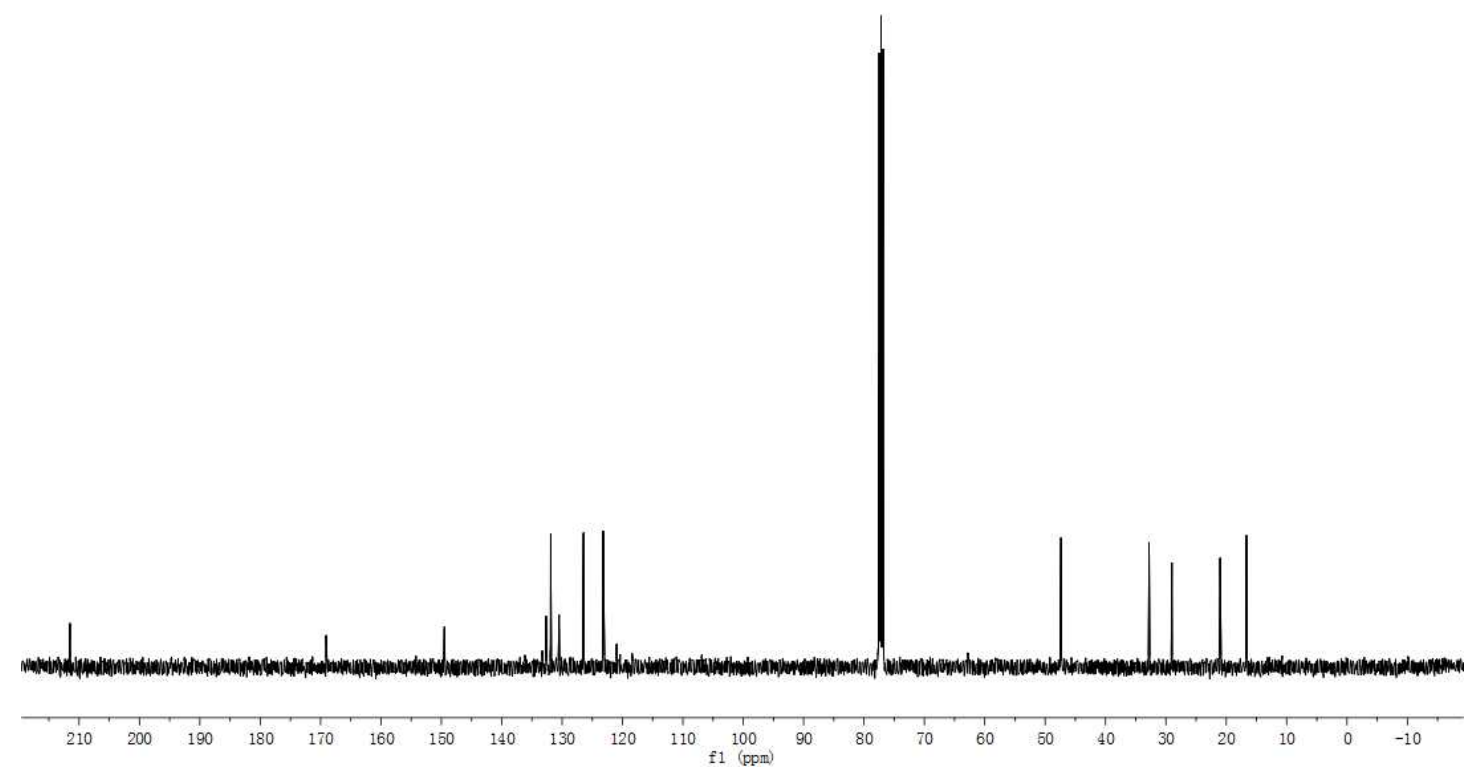




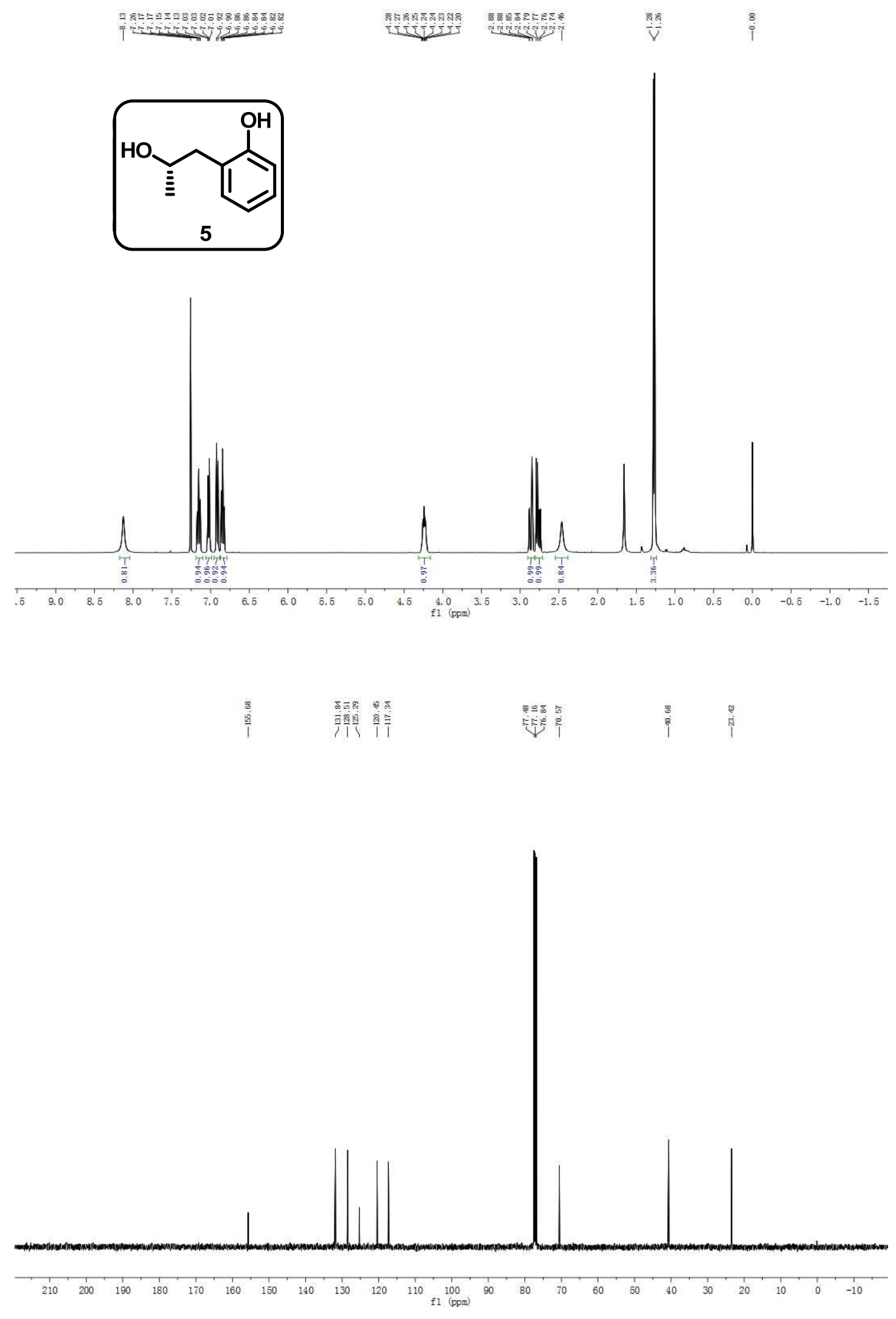



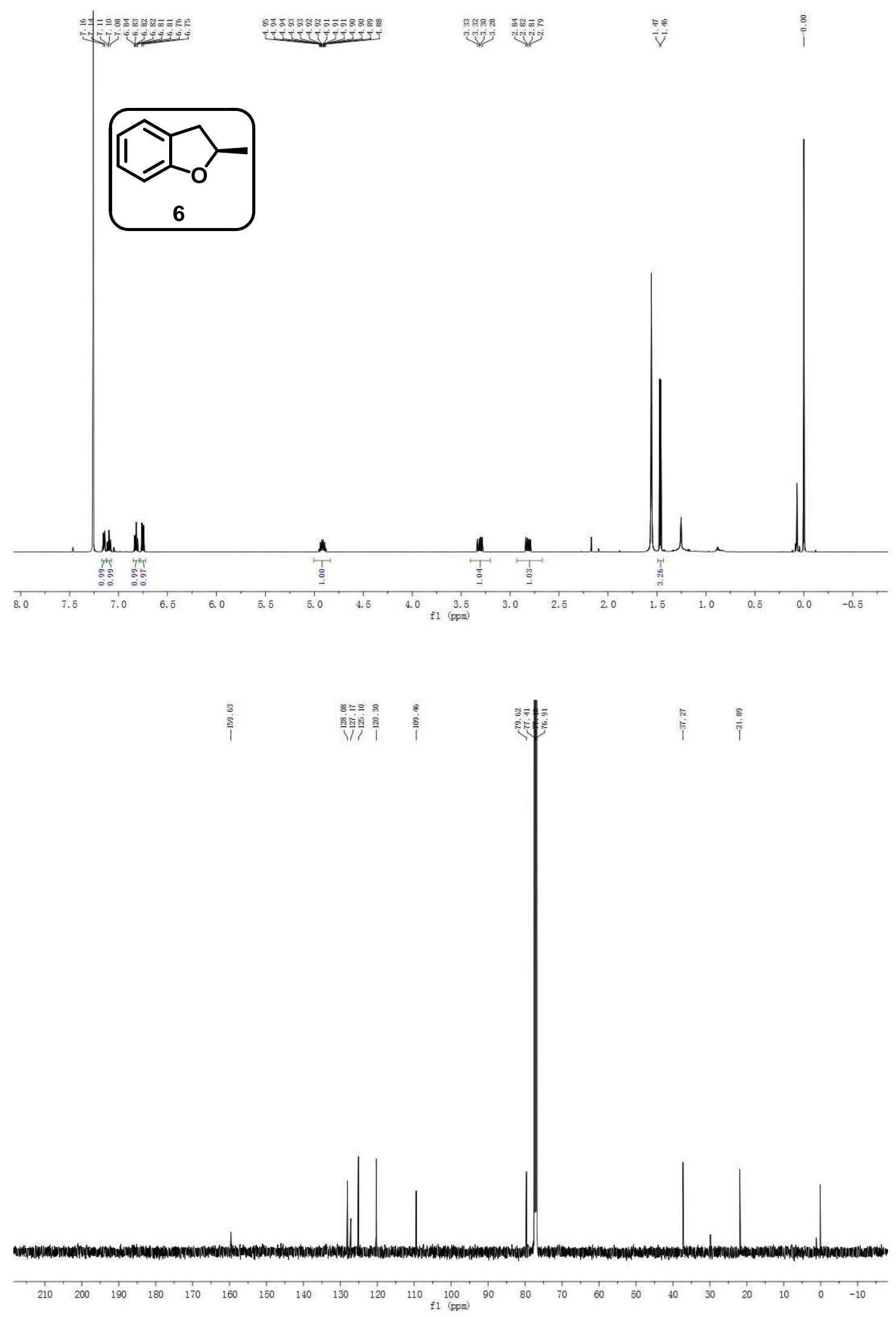

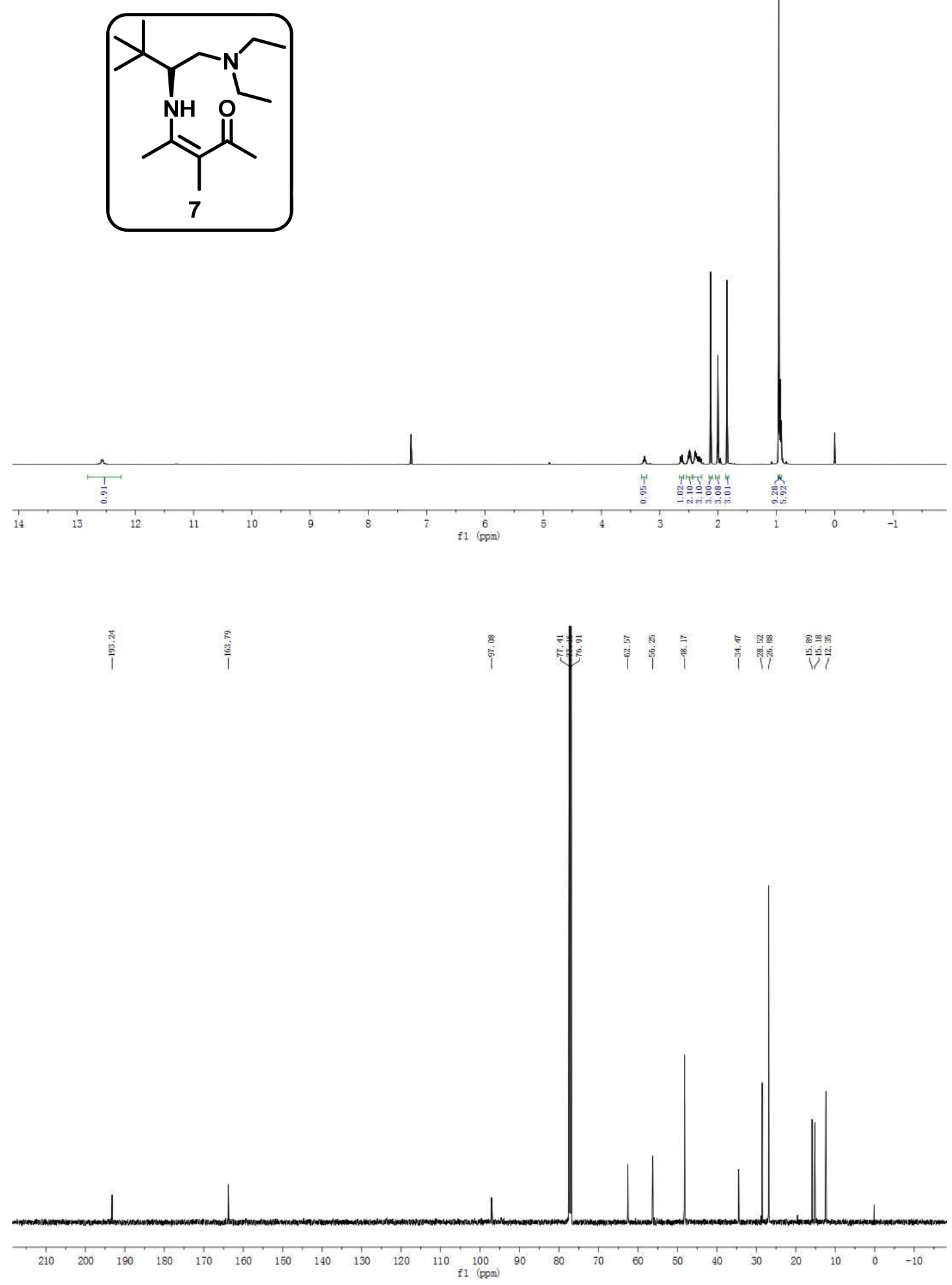

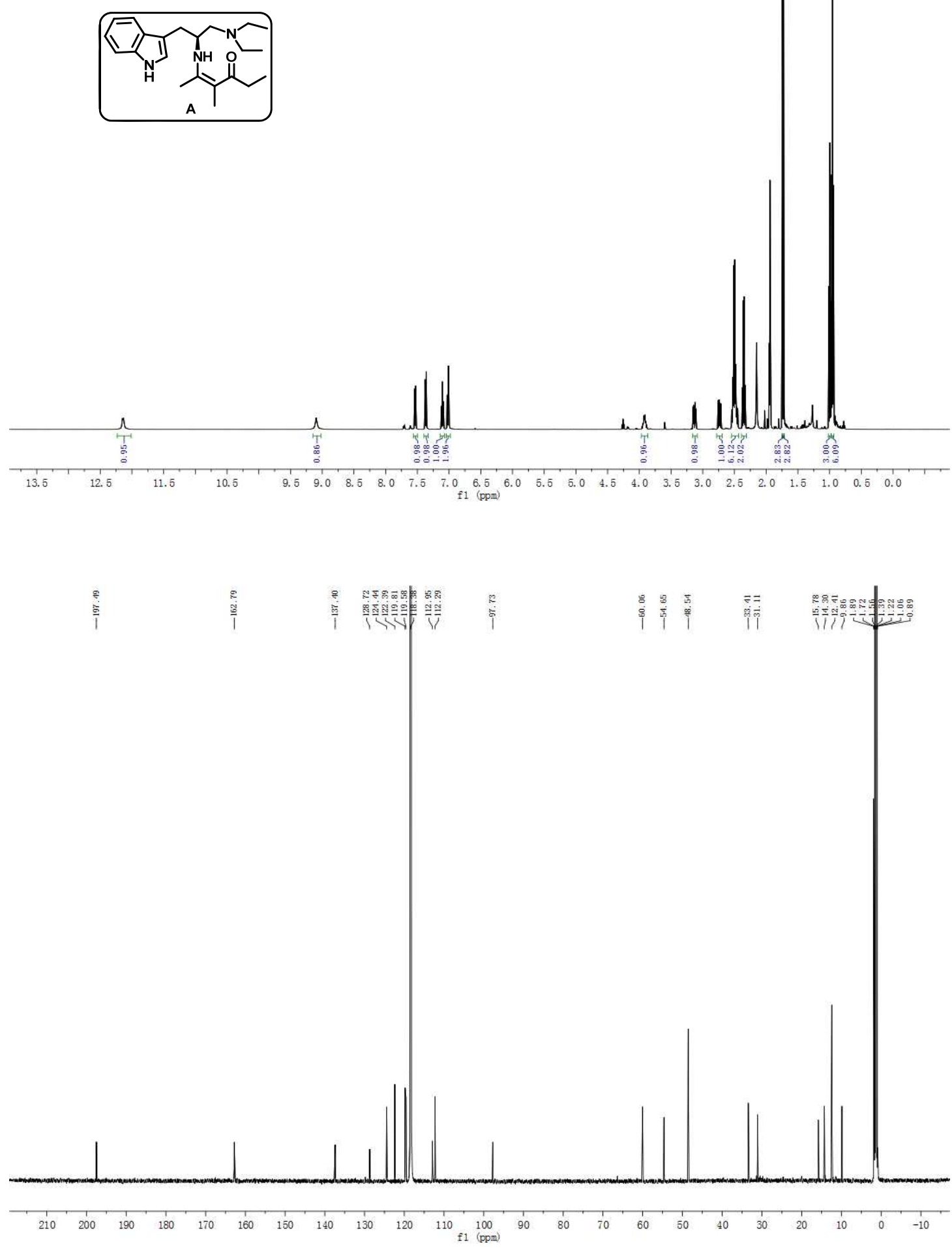


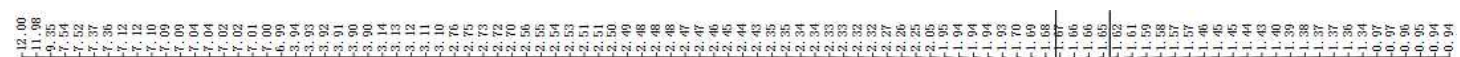
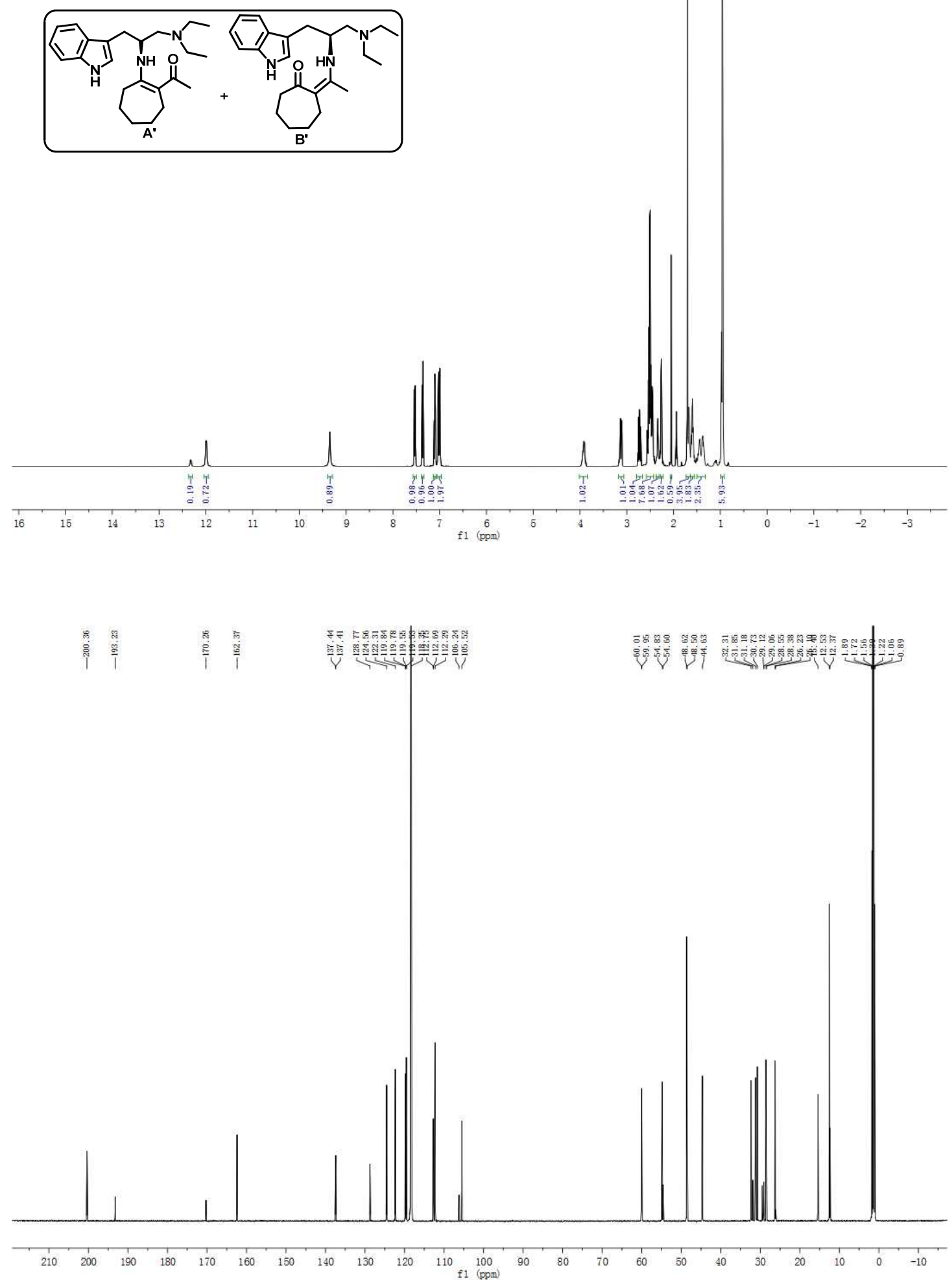


\section{HPLC charts}

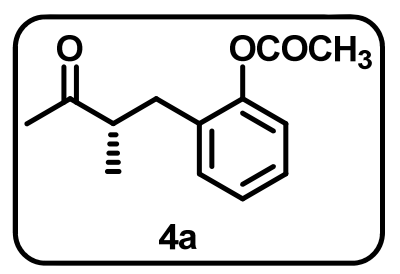

$\langle$ Chromatogram〉

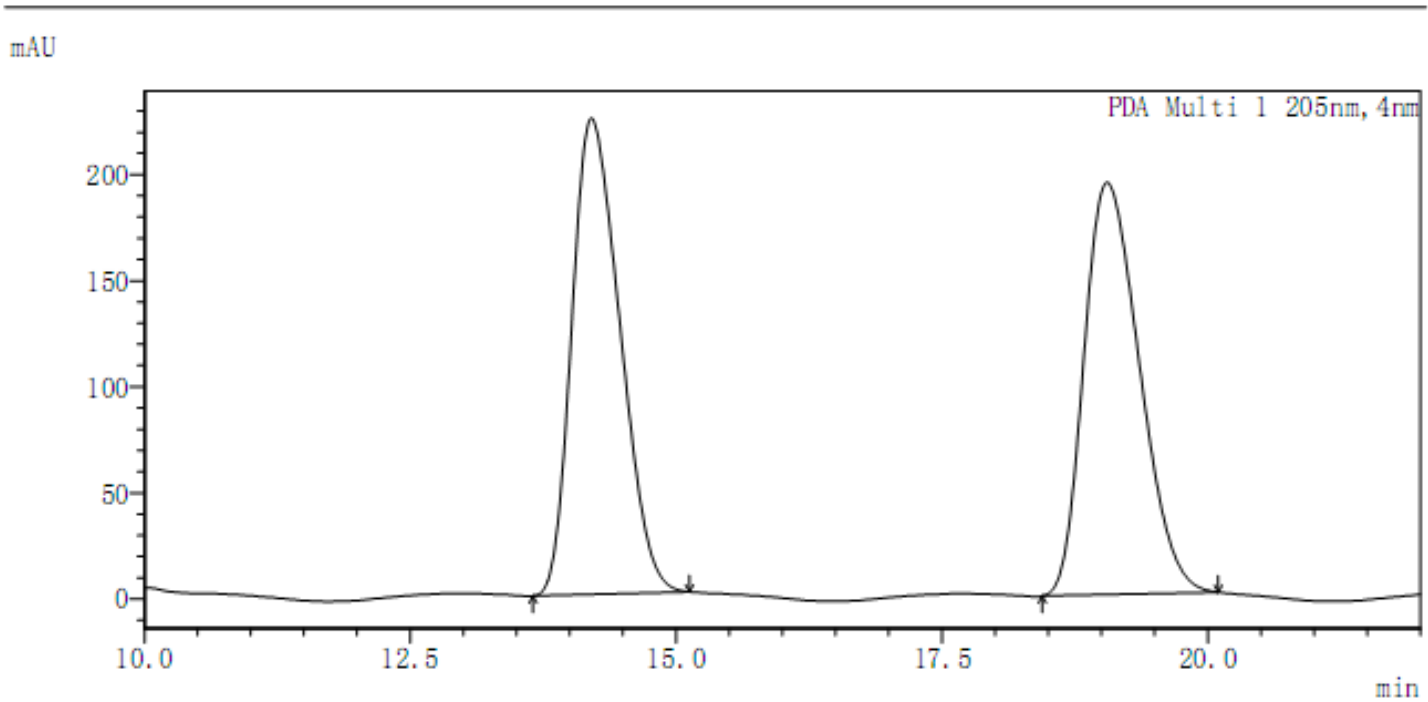

〈Peak Results〉

PDA Chl 205nm

\begin{tabular}{|c|c|c|c|c|}
\hline Index & Time/min & Height/mAU & Quantity/Area & Area \%/\% \\
\hline 1 & 14.204 & 224384 & 7056826 & 50.014 \\
\hline 2 & 19.053 & 194253 & 7052921 & 49.986 \\
\hline
\end{tabular}

$\langle$ Chromatogram〉

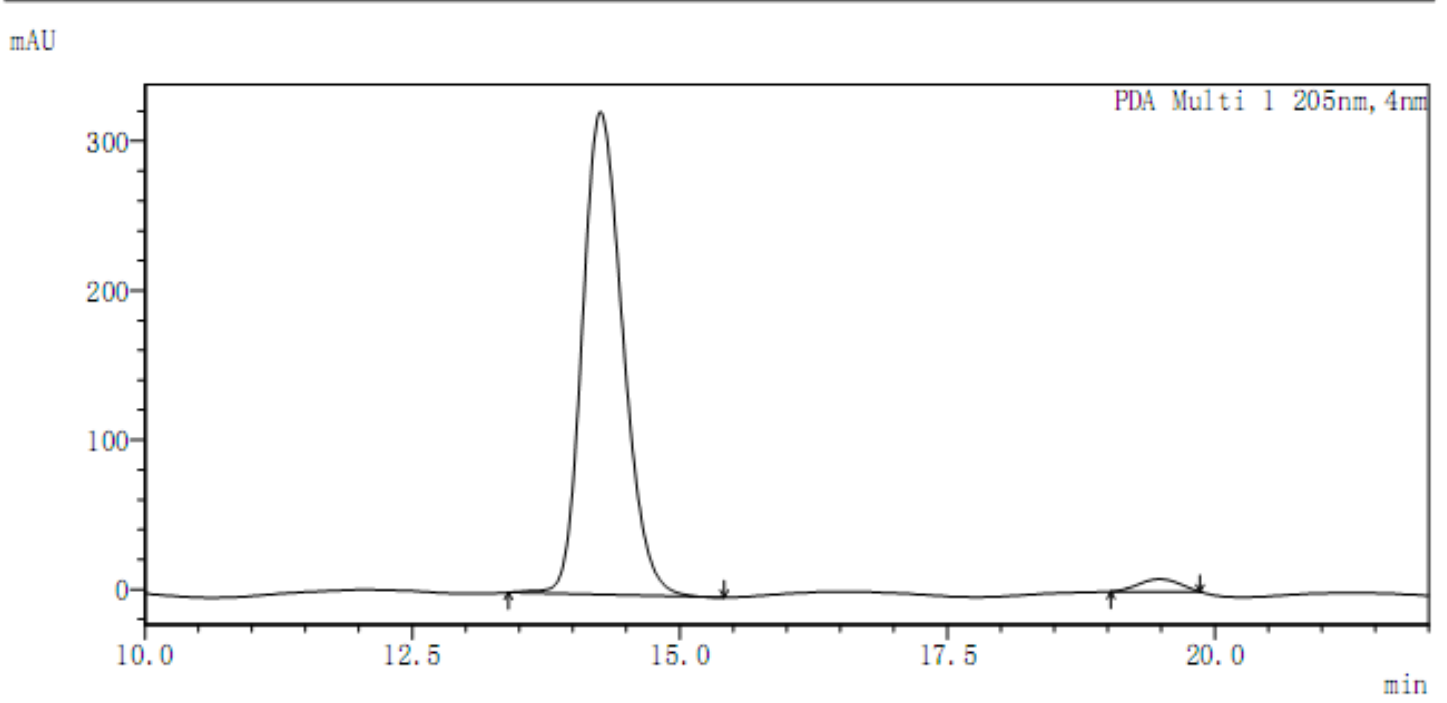

〈Peak Results>

PDA Chl 205nm

\begin{tabular}{|c|c|c|c|c|}
\hline Index & Time/min & Height/mAU & Quantity/Area & Area \%/\% \\
\hline 1 & 14.258 & 322657 & 8498734 & 97.433 \\
\hline 2 & 19.479 & 8419 & 223953 & 2.567 \\
\hline
\end{tabular}




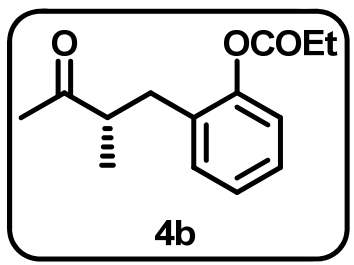

$\langle$ Chromatogram〉

UAU

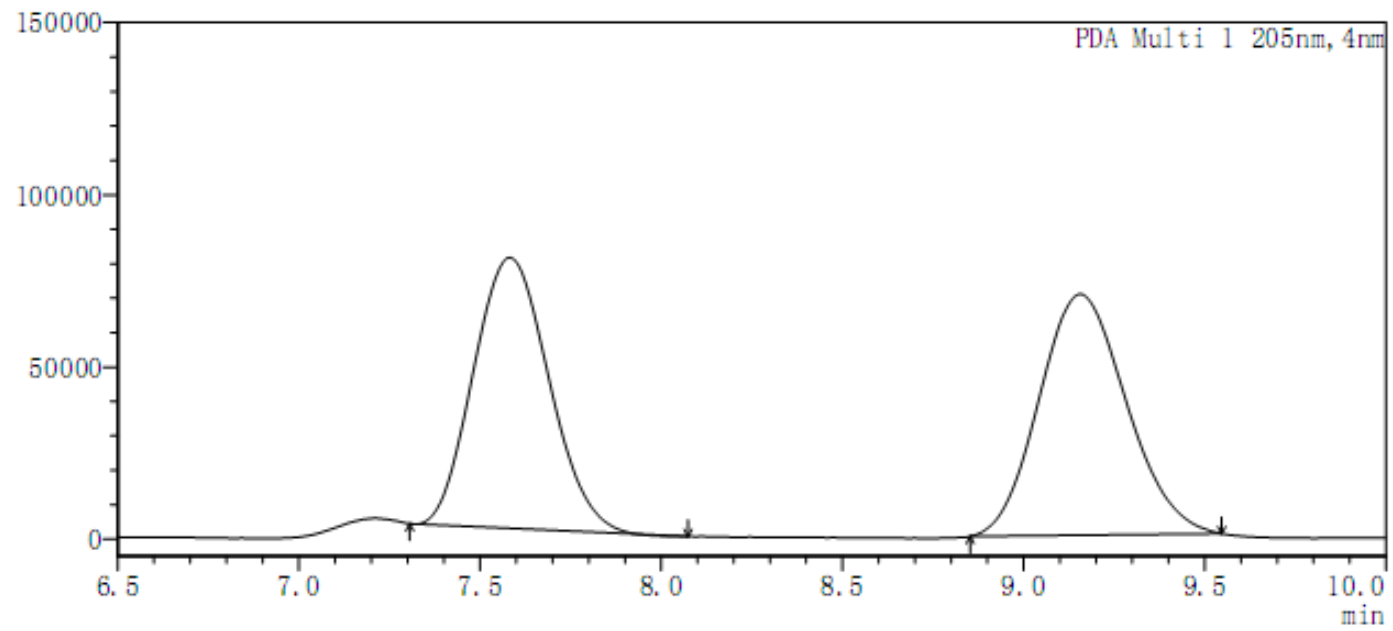

$\langle$ Peak Results>

PDA Chl 205nm

\begin{tabular}{|c|c|c|c|c|}
\hline Index & Time/min & Height/mAU & Quantity/Area & Area \%/\% \\
\hline 1 & 7.582 & 78745 & 1134462 & 49.219 \\
\hline 2 & 9.156 & 70005 & 1170471 & 50.781 \\
\hline
\end{tabular}

$\langle$ Chromatogram〉

mAU

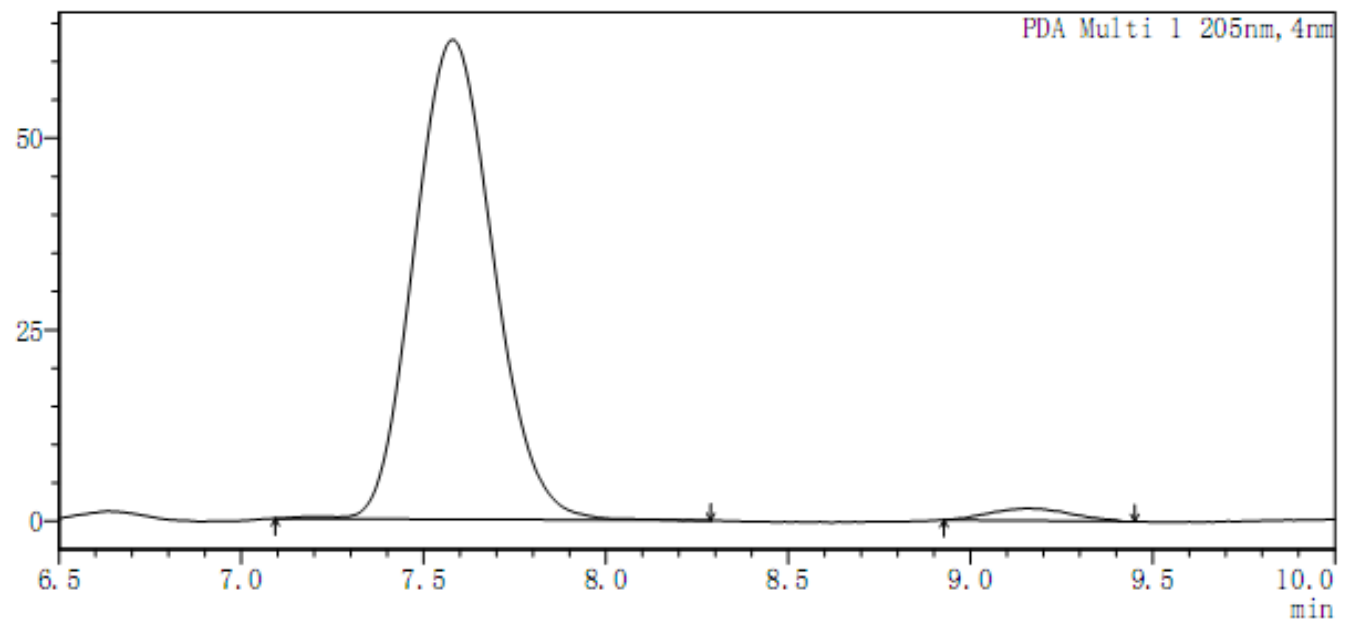

〈Peak Results>

PDA Ch1 205nm

\begin{tabular}{|c|c|c|c|c|}
\hline Index & Time/min & Height/mAU & Quantity/Area & Area \%/\% \\
\hline 1 & 7.580 & 62667 & 945925 & 97.446 \\
\hline 2 & 9.161 & 1624 & 24796 & 2.554 \\
\hline
\end{tabular}




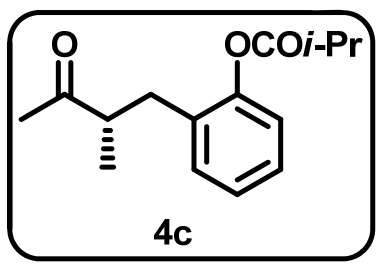

〈Chromatogram〉

mAU

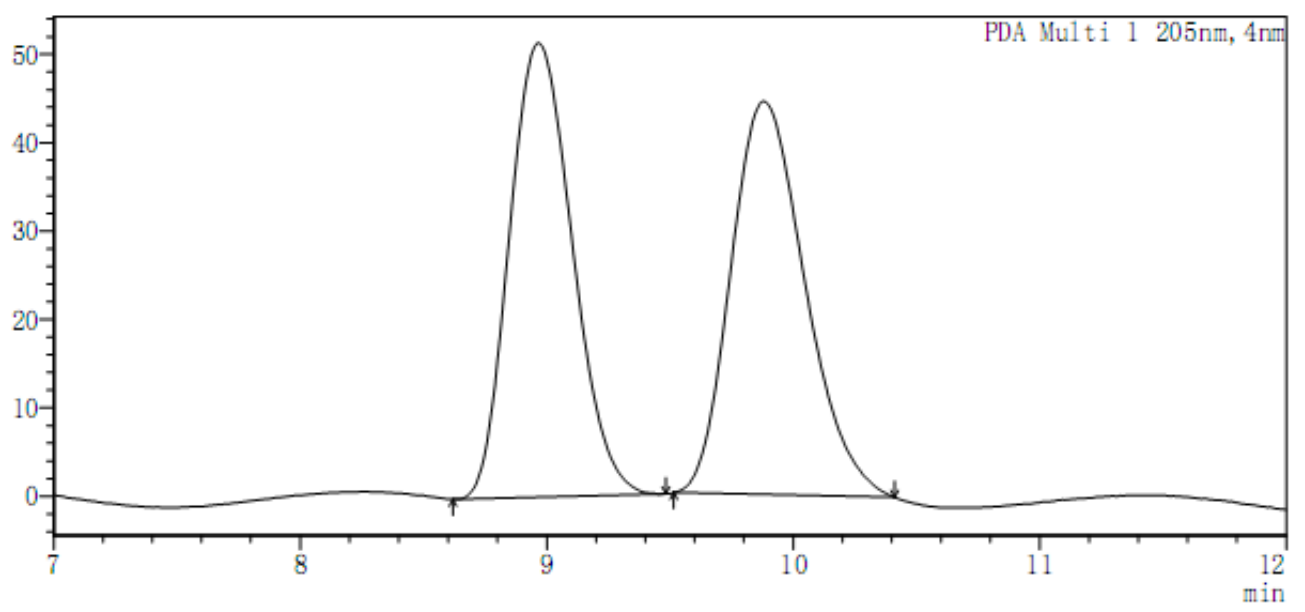

〈Peak Results>

PDA Ch1 205nm.

\begin{tabular}{|c|c|c|c|c|}
\hline Index & Time/min & Height/mAU & Quantity/Area & Area \%/\% \\
\hline 1 & 8.966 & 51368 & 912207 & 49.525 \\
\hline 2 & 9.880 & 44523 & 929724 & 50.475 \\
\hline
\end{tabular}

$\langle$ Chromatogram〉

mAU

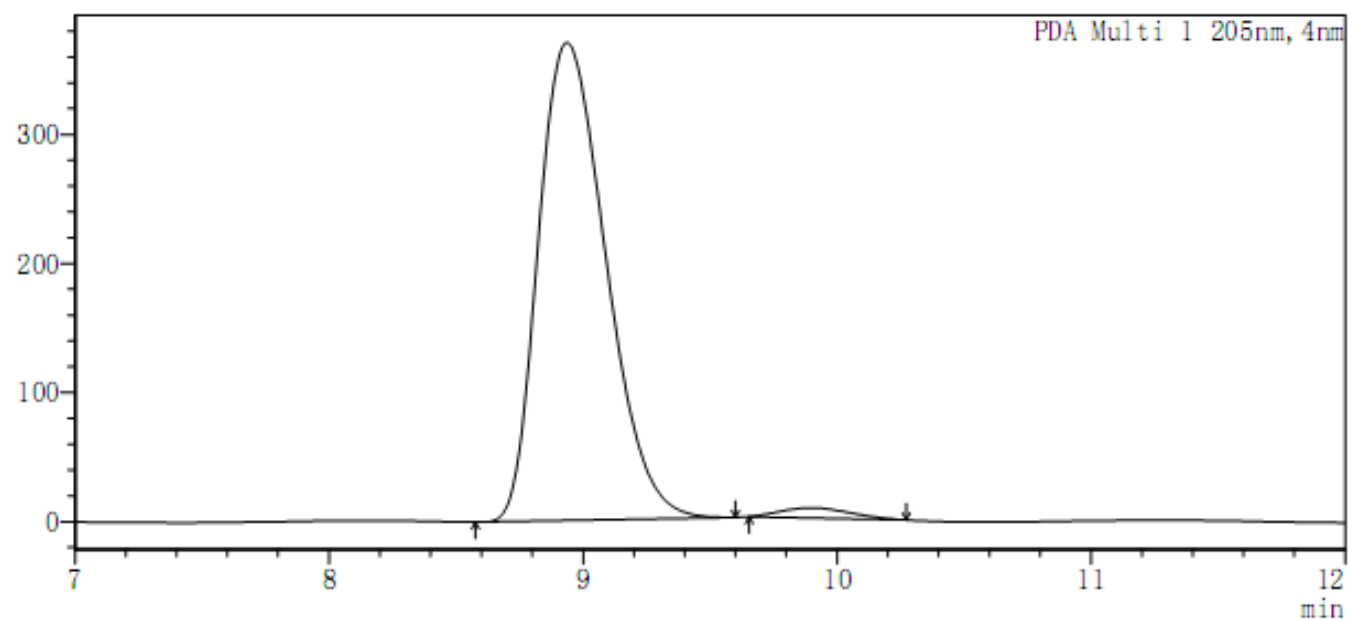

〈Peak Results>

PDA Ch1 205nm

\begin{tabular}{|c|c|c|c|c|}
\hline Index & Time/min & Height/mAU & Quantity/Area & Area \%/\% \\
\hline 1 & 8.936 & 370416 & 6842805 & 97.873 \\
\hline 2 & 9.893 & 7774 & 148679 & 2.127 \\
\hline
\end{tabular}




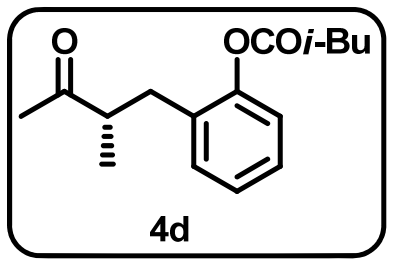

$\langle$ Chromatogram〉

mAU

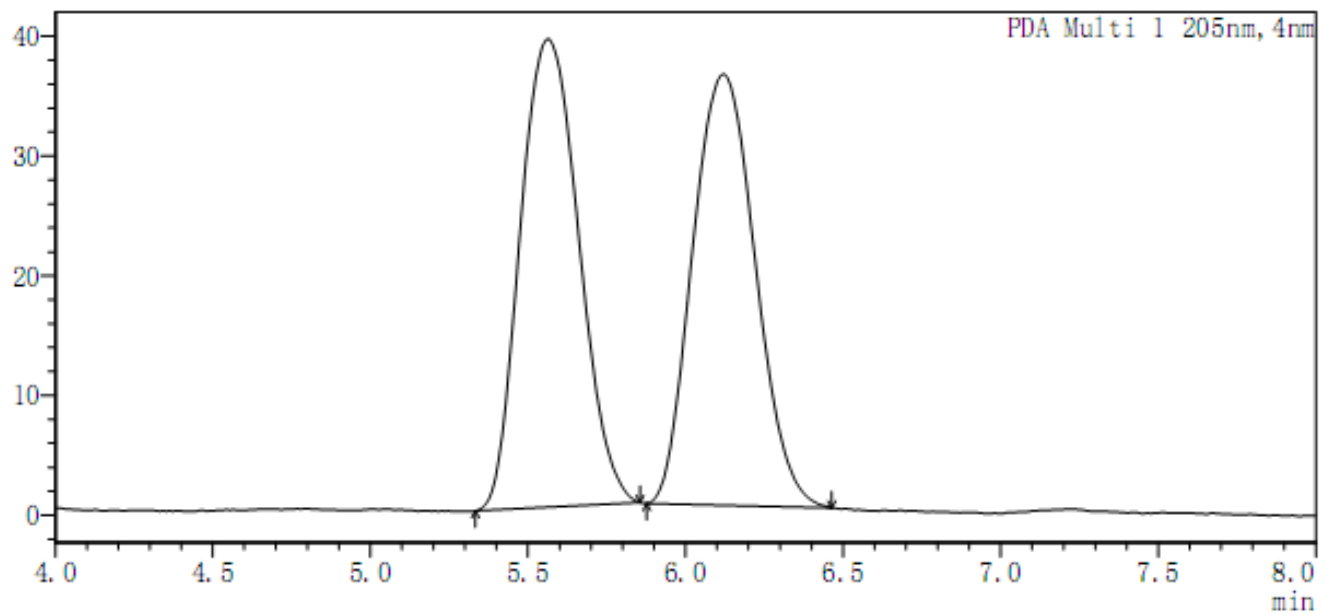

〈Peak Results〉

PDA Ch1 205nm

\begin{tabular}{|c|c|c|c|c|}
\hline Index & Time/min & Height/mAU & Quantity/Area & Area \%/\% \\
\hline 1 & 5.564 & 39084 & 505673 & 50.324 \\
\hline 2 & 6.120 & 36030 & 499160 & 49.676 \\
\hline
\end{tabular}

$\langle$ Chromatogram〉

$\mathrm{mAU}$

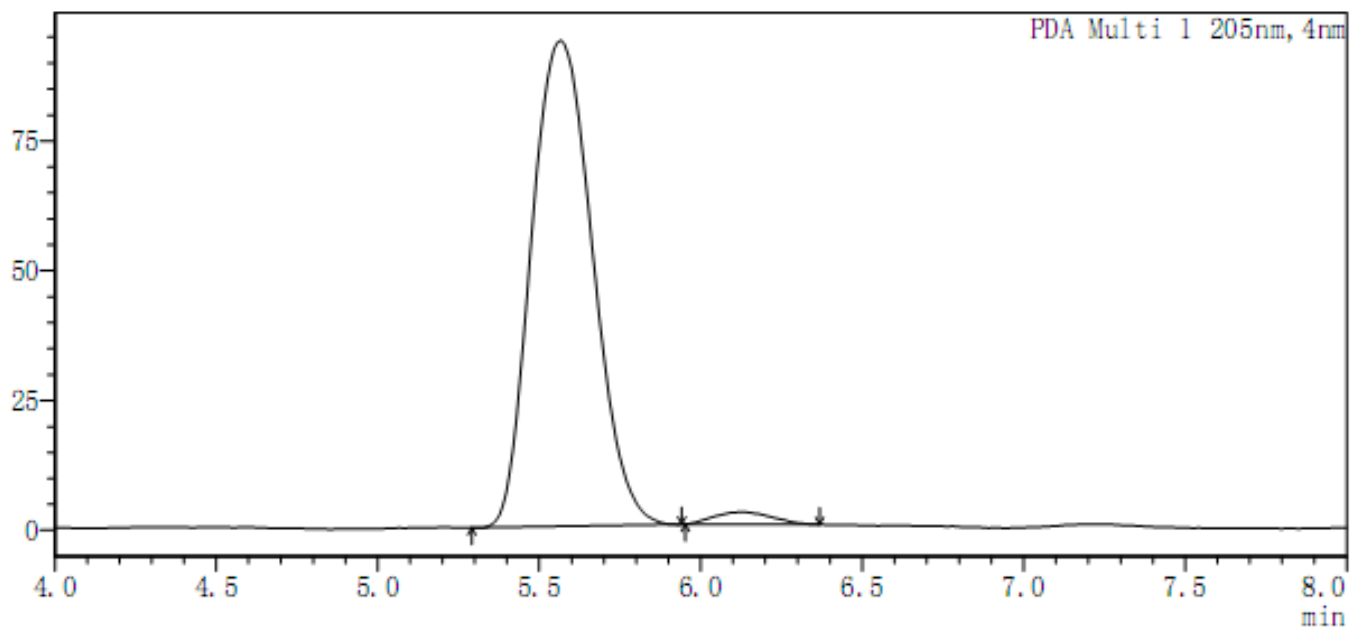

〈Peak Results>

PDA Chl $205 \mathrm{~nm}$

\begin{tabular}{|c|c|c|c|c|}
\hline Index & Time/min & Height/mAU & Quantity/Area & Area \%/\% \\
\hline 1 & 5.566 & 93611 & 1231639 & 97.664 \\
\hline 2 & 6.132 & 2403 & 29455 & 2.336 \\
\hline
\end{tabular}




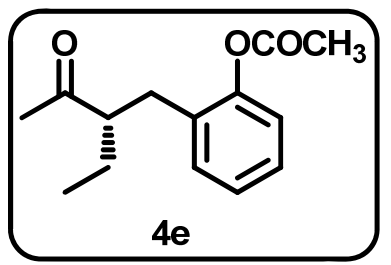

$\langle$ Chromatogram〉

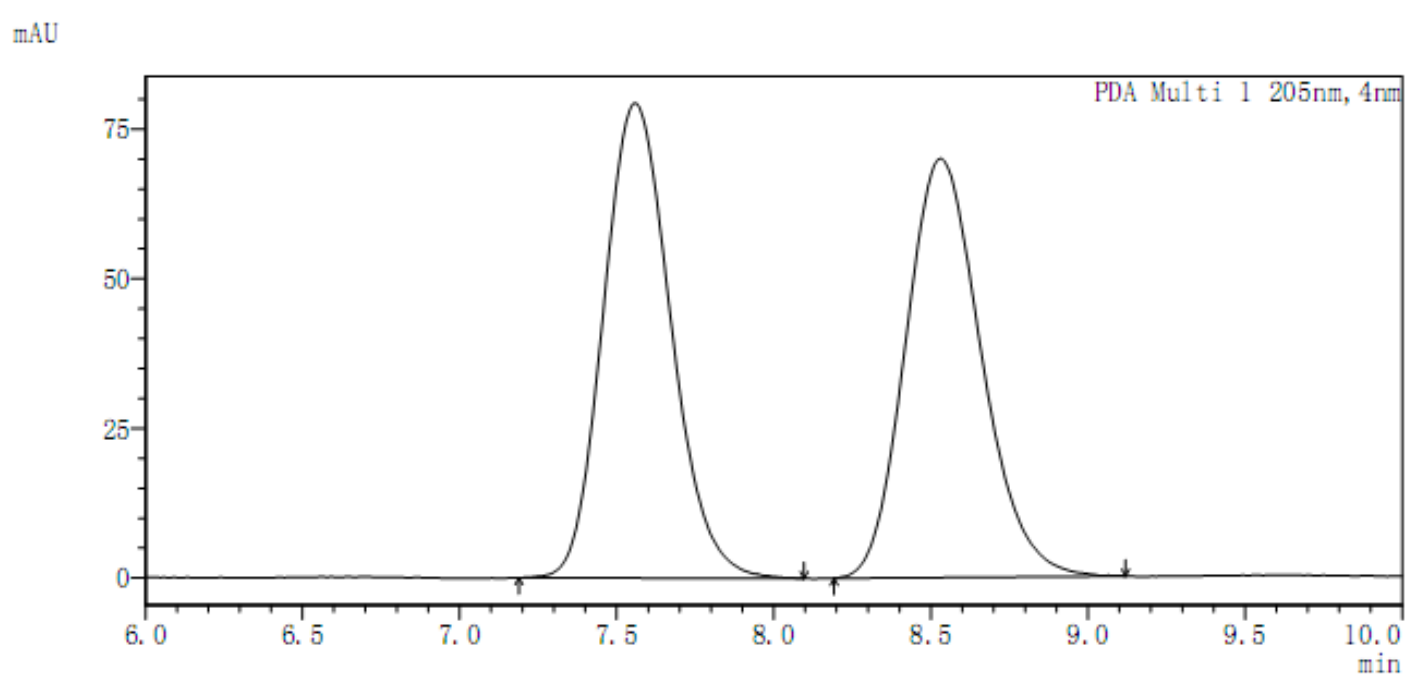

〈Peak Results〉

PDA Ch1 205nm

\begin{tabular}{|c|c|c|c|c|}
\hline Index & Time/min & Height/mAU & Quantity/Area & Area \%/\% \\
\hline 1 & 7.559 & 79378 & 1186739 & 50.283 \\
\hline 2 & 8.531 & 70054 & 1173375 & 49.717 \\
\hline
\end{tabular}

〈Chromatogram〉

$\mathrm{mAU}$

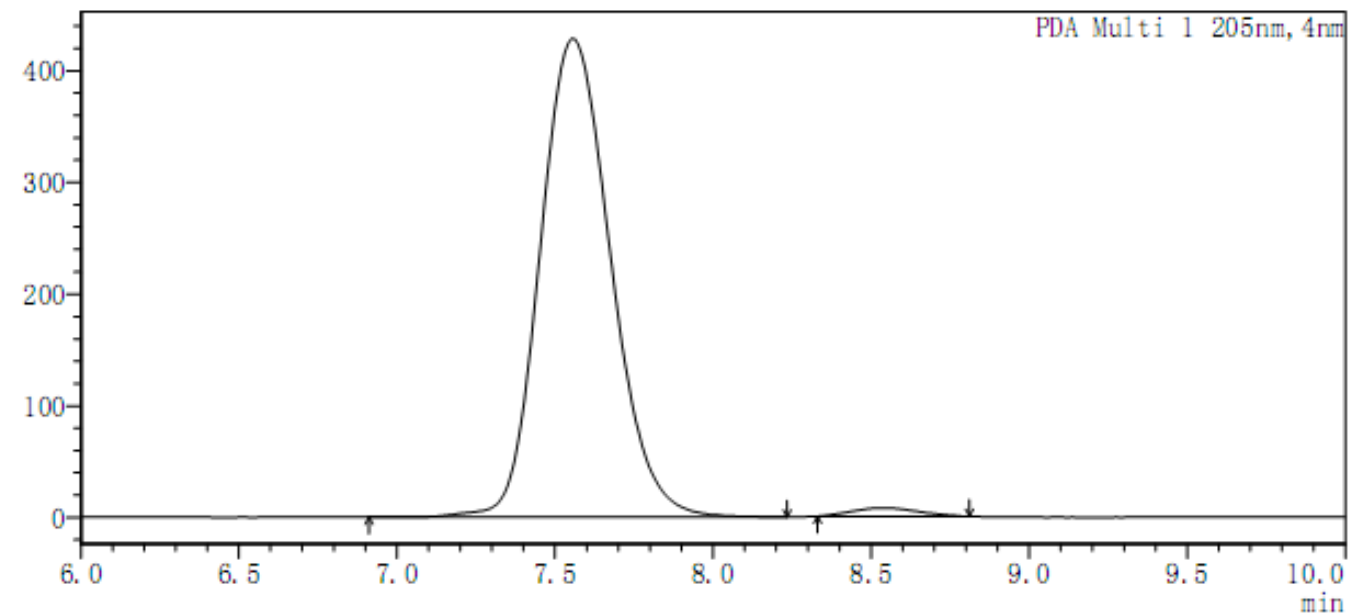

〈Peak Results〉

PDA Chl 205nm

\begin{tabular}{|c|c|c|c|c|}
\hline Index & Time/min & Height/mAU & Quantity/Area & Area \%/\% \\
\hline 1 & 7.557 & 428367 & 6640737 & 98.429 \\
\hline 2 & 8.538 & 7228 & 105985 & 1.571 \\
\hline
\end{tabular}




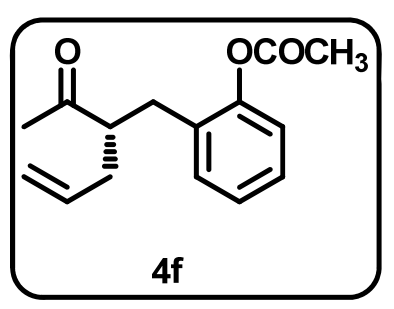

$\langle$ Chromatogram〉

mAU

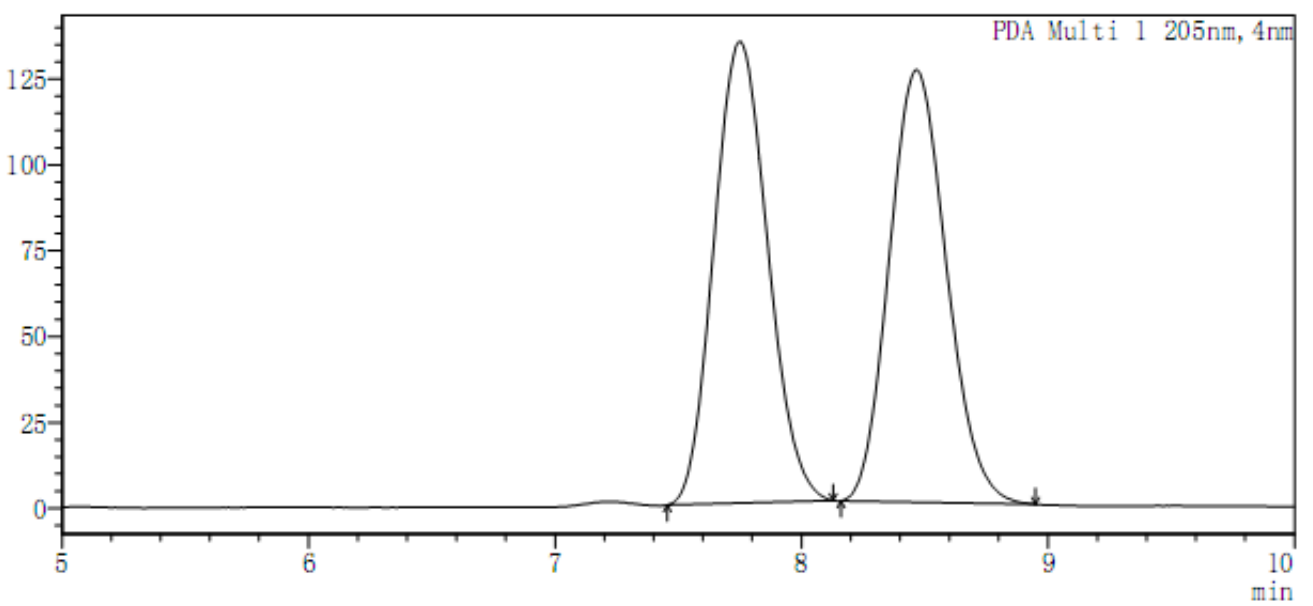

〈Peak Results>

PDA Chl $205 \mathrm{~nm}$

\begin{tabular}{|c|c|c|c|c|}
\hline Index & Time/min & Height/mAU & Quantity/Area & Area \%/\% \\
\hline 1 & 7.750 & 134357 & 2039989 & 50.082 \\
\hline 2 & 8.466 & 125935 & 2033327 & 49.918 \\
\hline
\end{tabular}

$\langle$ Chromatogram〉

mAU

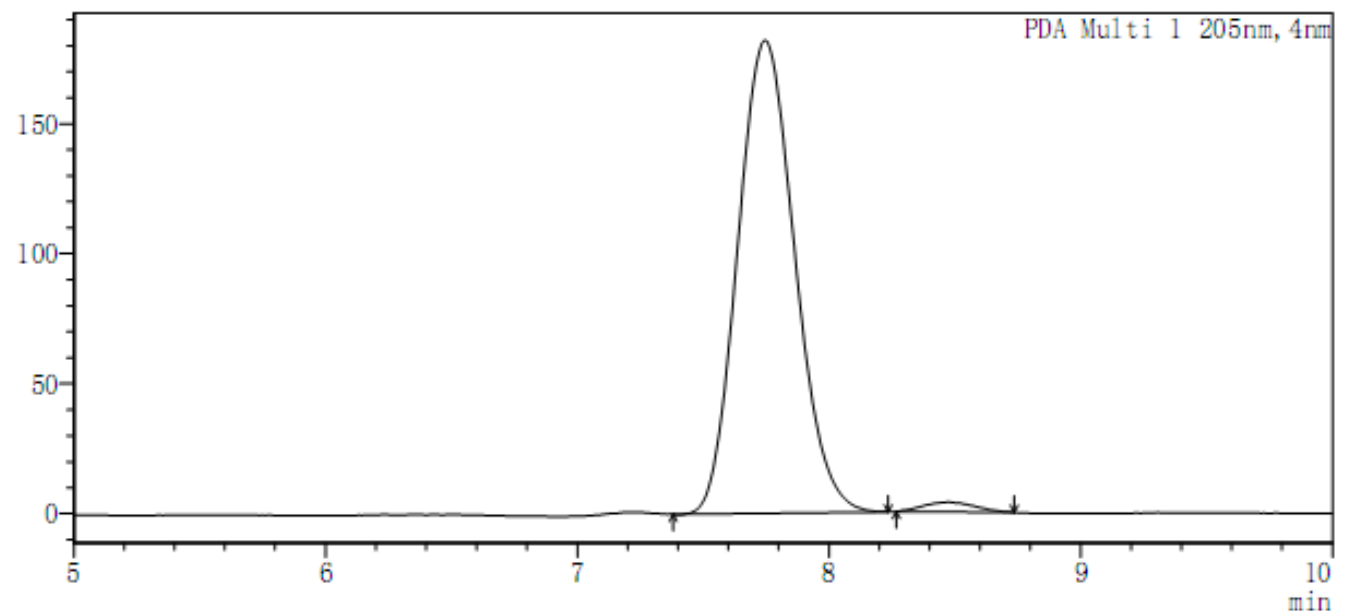

〈Peak Results>

PDA Ch1 $205 \mathrm{~nm}$

\begin{tabular}{|c|c|c|c|c|}
\hline Index & Time/min & Height/mAU & Quantity/Area & Area \%/\% \\
\hline 1 & 7.746 & 181937 & 2863752 & 98.192 \\
\hline 2 & 8.471 & 3704 & 52721 & 1.808 \\
\hline
\end{tabular}




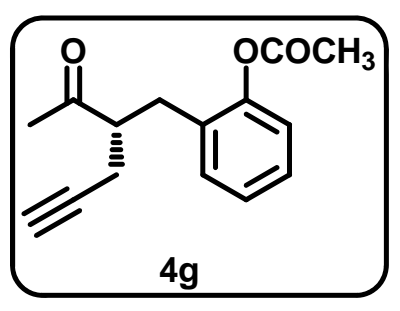

$\langle$ Chromatogram〉

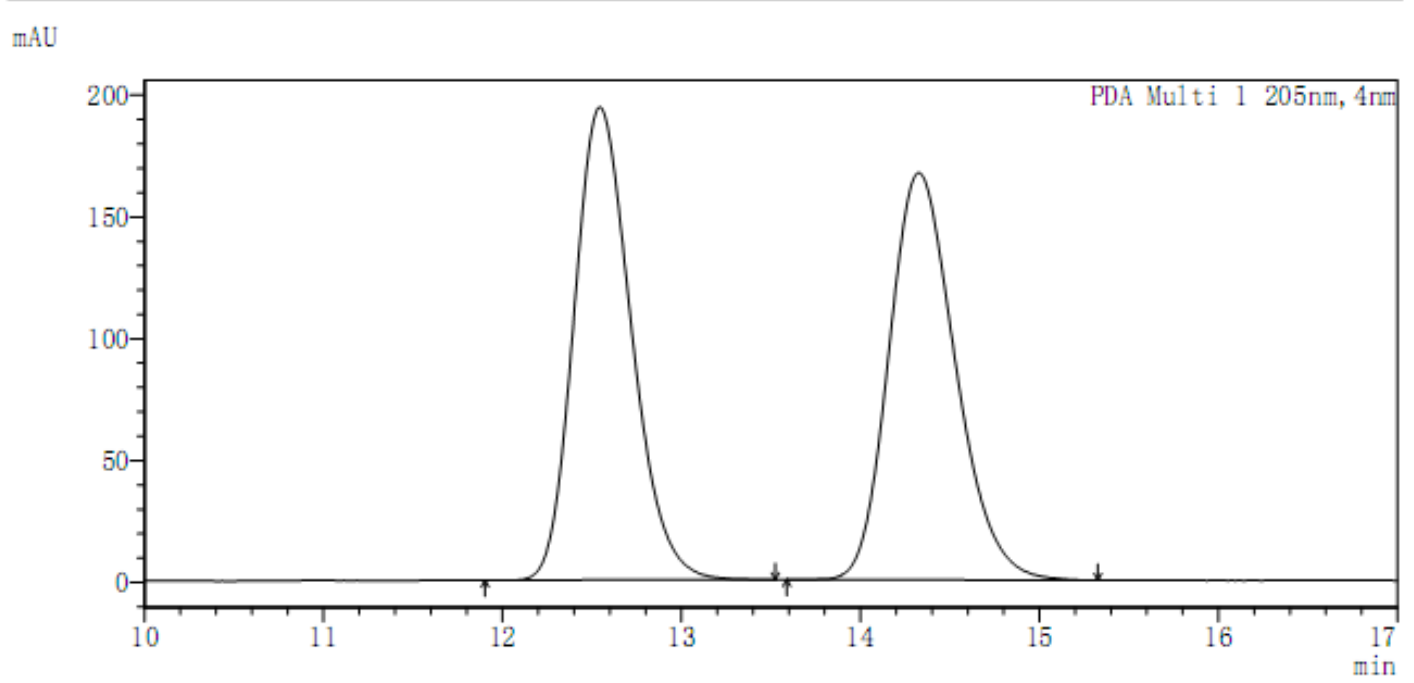

〈Peak Results〉

PDA Ch1 205nm

\begin{tabular}{|c|c|c|c|c|}
\hline Index & Time/min & Height/mAU & Quantity/Area & Area \%/\% \\
\hline 1 & 12.545 & 194123 & 4309413 & 49.994 \\
\hline 2 & 14.326 & 167179 & 4310400 & 50.006 \\
\hline
\end{tabular}

$\langle$ Chromatogram〉

$\mathrm{mAU}$

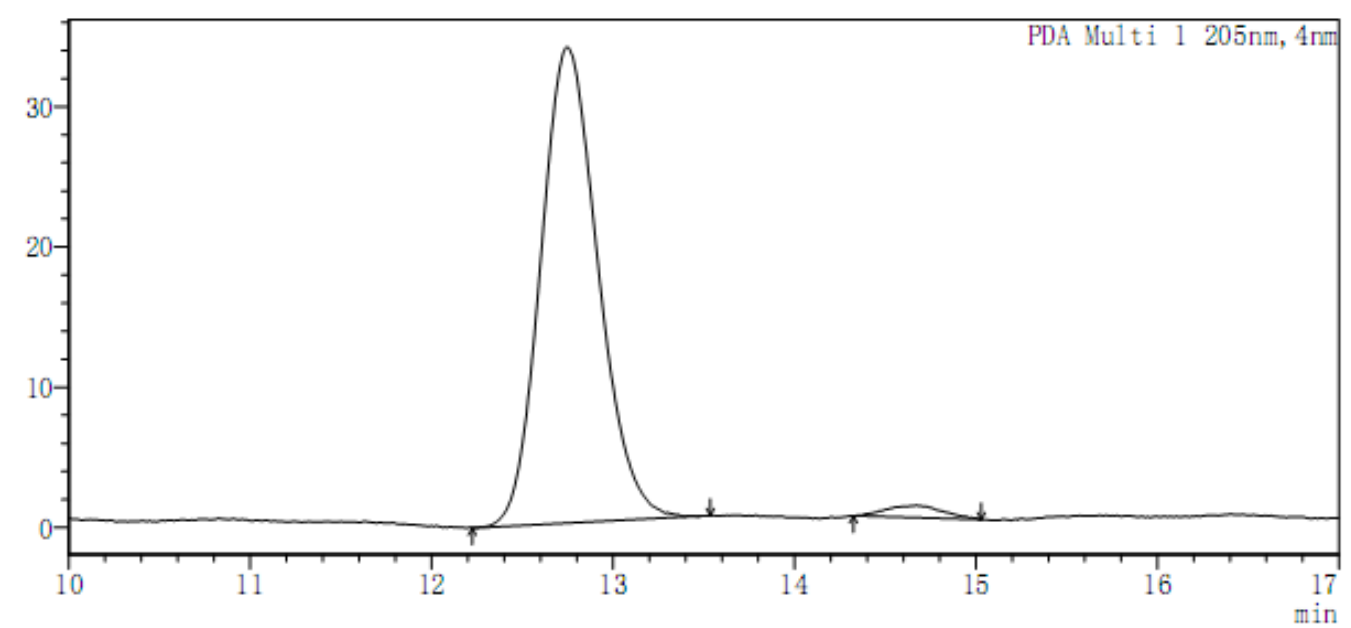

〈Peak Results〉

PDA Ch1 205nm

\begin{tabular}{|c|c|c|c|c|}
\hline Index & Time/min & Height/mAU & Quanti ty/Area & Area \%/\% \\
\hline 1 & 12.748 & 33940 & 746401 & 97.538 \\
\hline 2 & 14.673 & 863 & 18844 & 2.462 \\
\hline
\end{tabular}




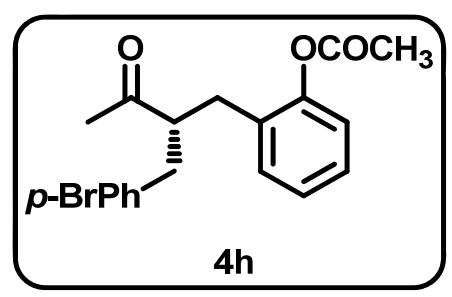

$\langle$ Chromatogram〉

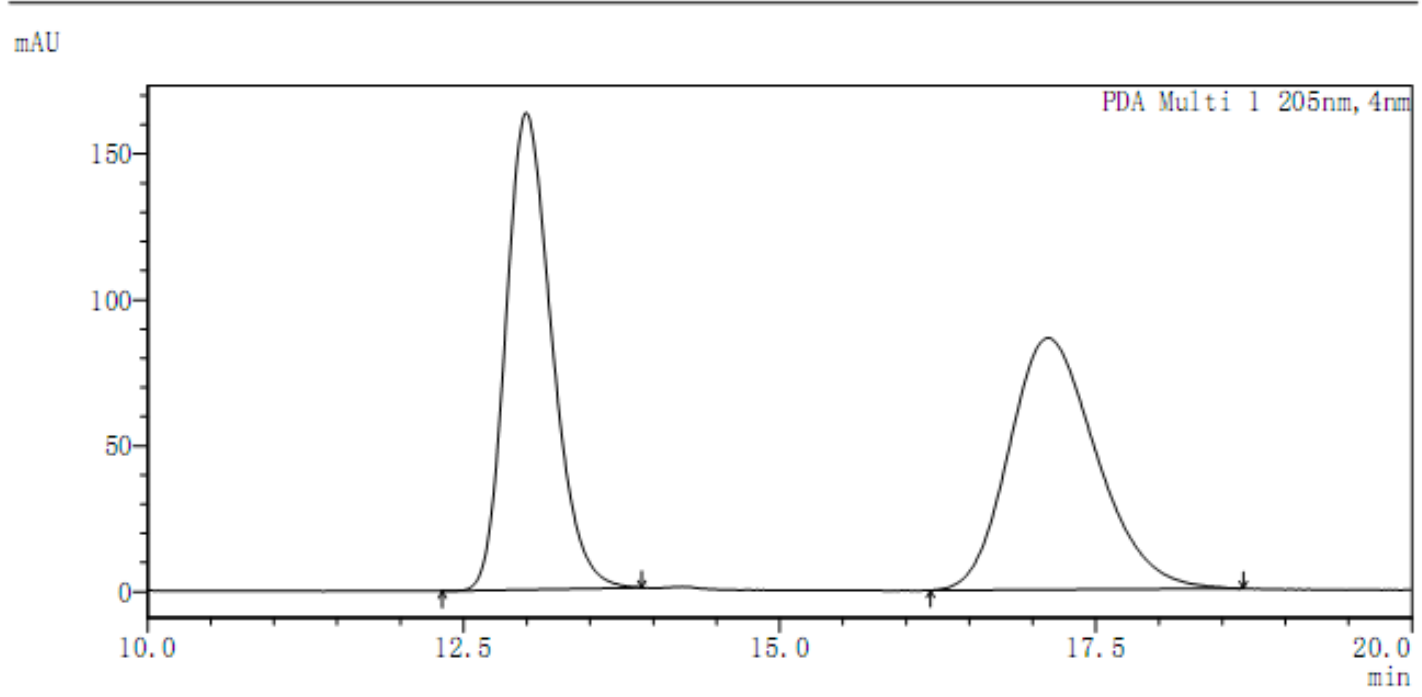

〈Peak Results〉

PDA Chl 205nm

\begin{tabular}{|c|c|c|c|c|}
\hline Index & Time/min & Height/mAU & Quantity/Area & Area \%/\% \\
\hline 1 & 12.994 & 163425 & 4134607 & 49.890 \\
\hline 2 & 17.122 & 86208 & 4152847 & 50.110 \\
\hline
\end{tabular}

$\langle$ Chromatogram〉

mAU

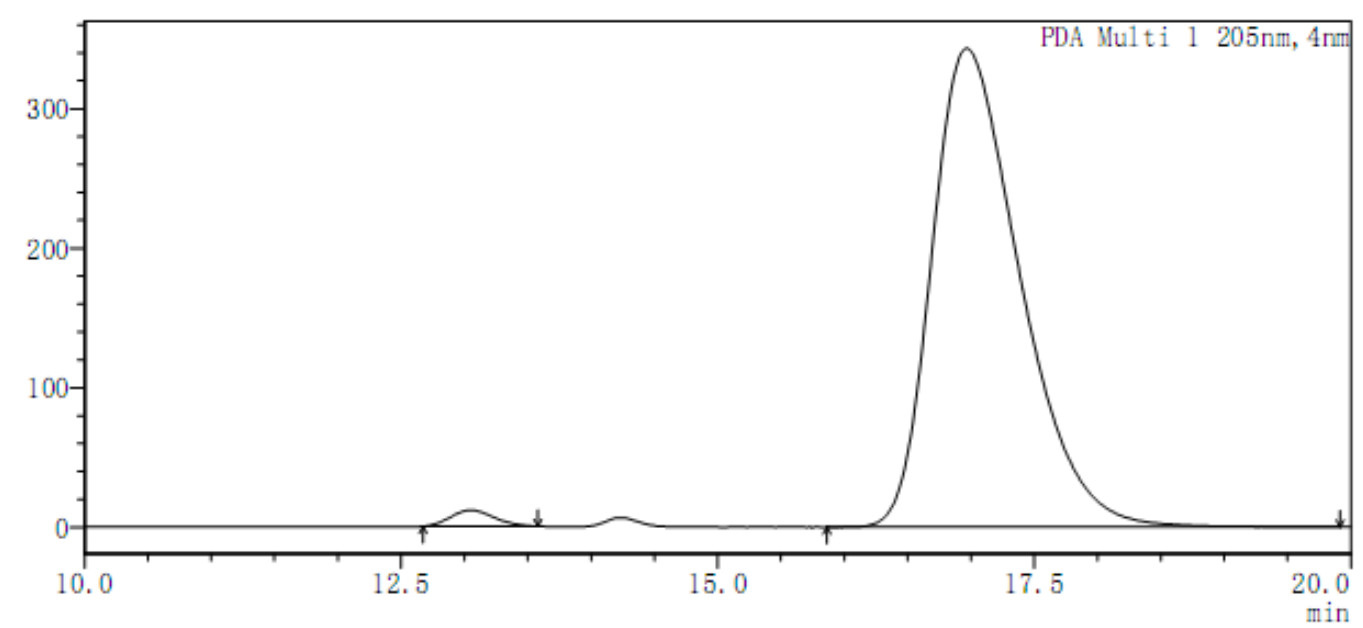

〈Peak Results〉

PDA Chl 205nm

\begin{tabular}{|c|c|c|c|c|}
\hline Index & Time/min & Height/mAU & Quantity/Area & Area \%/\% \\
\hline 1 & 13.047 & 11493 & 274650 & 1.625 \\
\hline 2 & 16.965 & 343231 & 16627564 & 98.375 \\
\hline
\end{tabular}




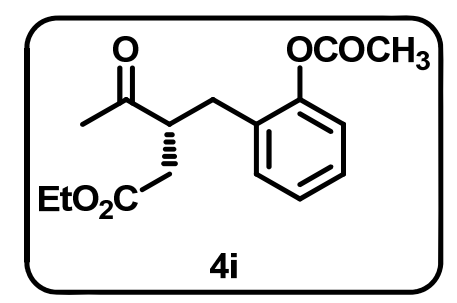

$\langle$ Chromatogram〉

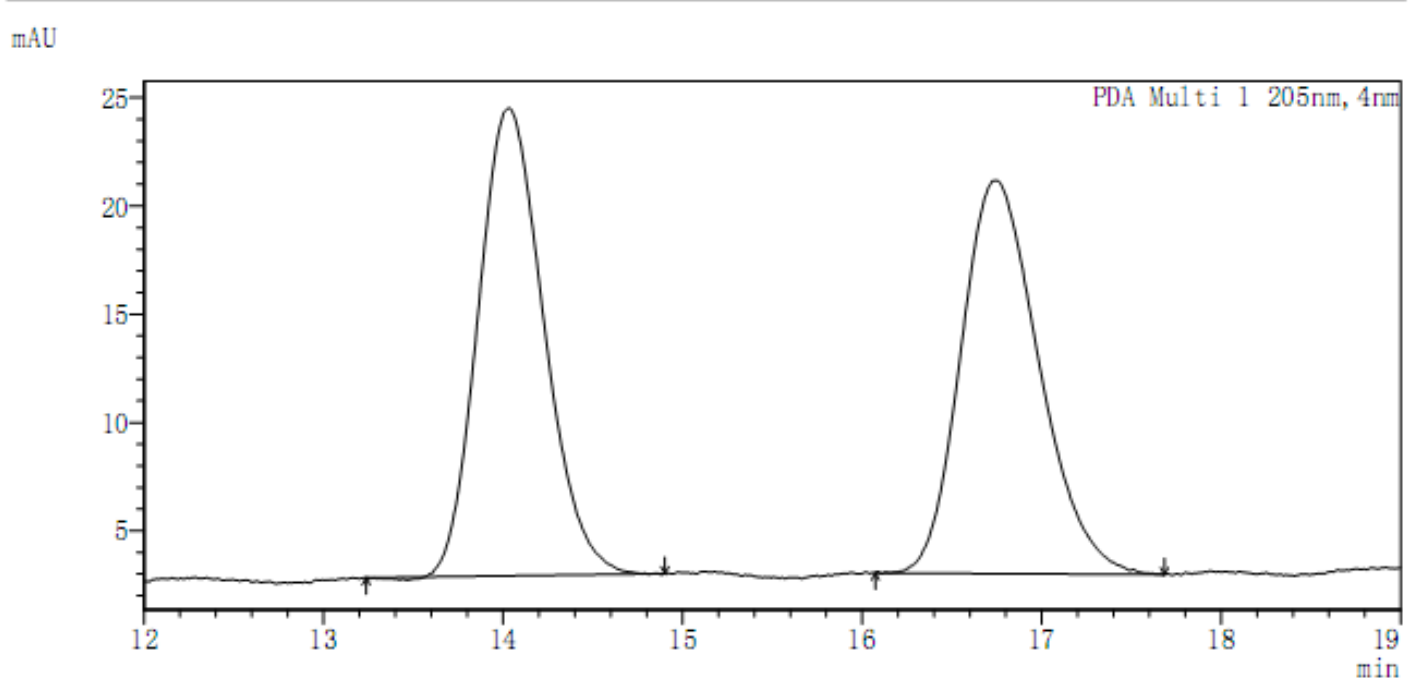

〈Peak Results>

PDA Chl $205 \mathrm{~nm}$

\begin{tabular}{|c|c|c|c|c|}
\hline Index & Time/min & Height/mAU & Quantity/Area & Area \%/\% \\
\hline 1 & 14.034 & 21607 & 547486 & 49.882 \\
\hline 2 & 16.749 & 18161 & 550079 & 50.118 \\
\hline
\end{tabular}

$\langle$ Chromatogram〉

mAU

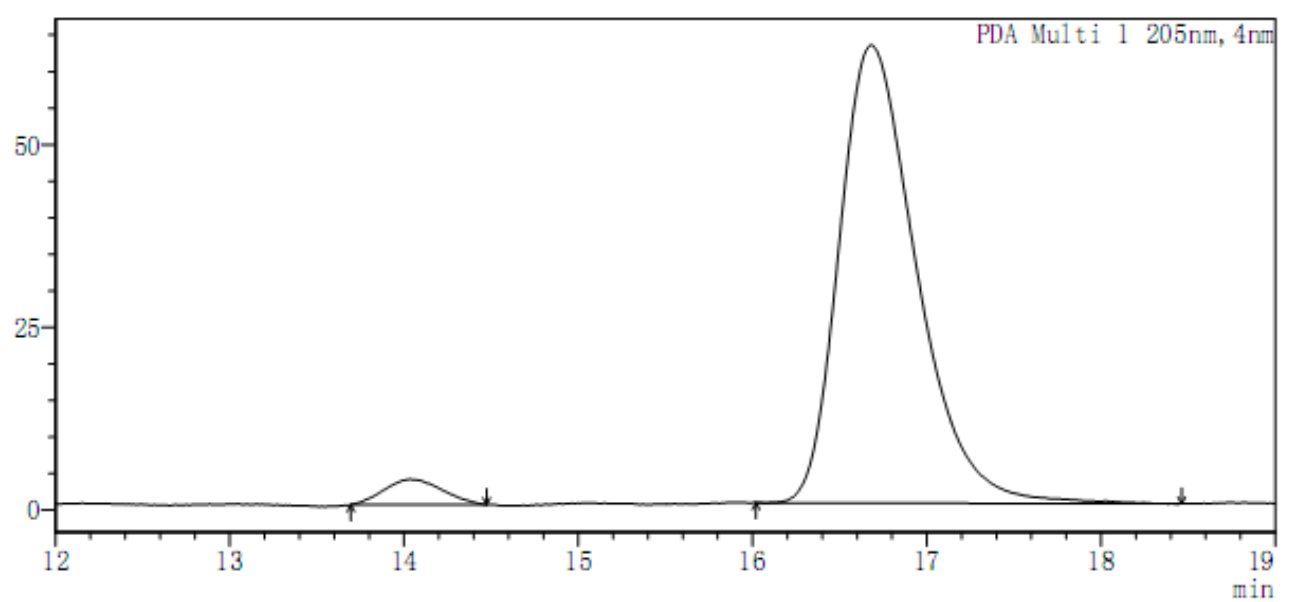

〈Peak Results>

PDA Ch1 205nm

\begin{tabular}{|c|c|c|c|c|}
\hline Index & Time/min & Height/mAU & Quantity/Area & Area \%/\% \\
\hline 1 & 14.037 & 3486 & 80510 & 3.995 \\
\hline 2 & 16.681 & 62659 & 1934668 & 96.005 \\
\hline
\end{tabular}




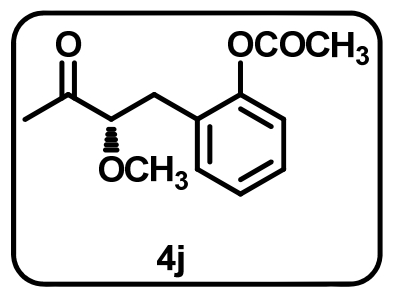

$\langle$ Chromatogram〉

UAL

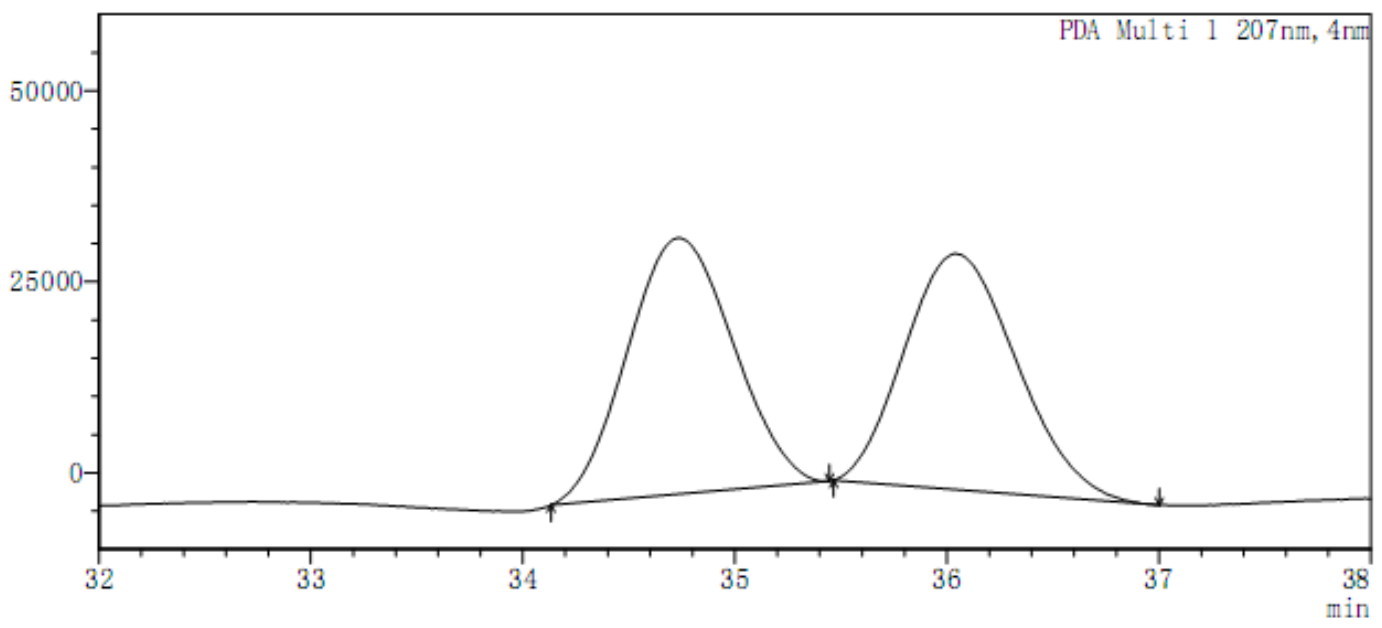

〈Peak Results〉

PDA Ch1 207nm

\begin{tabular}{|c|c|c|c|c|}
\hline Index & Time/min & Height/mAU & Quantity/Area & Area \%/\% \\
\hline 1 & 34.737 & 33517 & 1163174 & 50.656 \\
\hline 2 & 36.041 & 30876 & 1133057 & 49.344 \\
\hline
\end{tabular}

$\langle$ Chromatogram〉

mAU

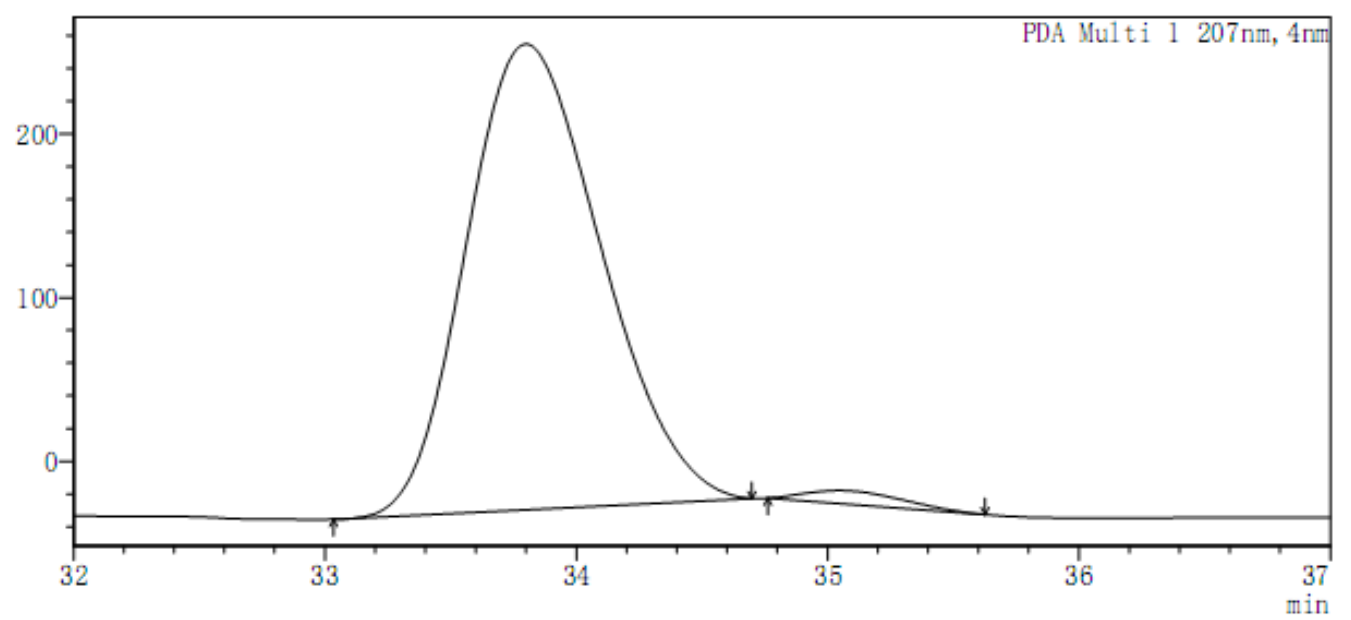

〈Peak Results〉

PDA Chl 207nm

\begin{tabular}{|c|c|c|c|c|}
\hline Index & Time/min & Height/mAU & Quant ity/Area & Area \%/\% \\
\hline 1 & 33.800 & 284357 & 10595699 & 97.985 \\
\hline 2 & 35.044 & 7852 & 217875 & 2.015 \\
\hline
\end{tabular}




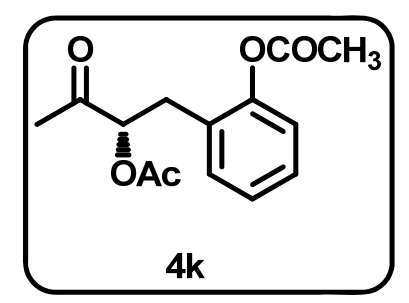

$\langle$ Chromatogram〉

IIAU

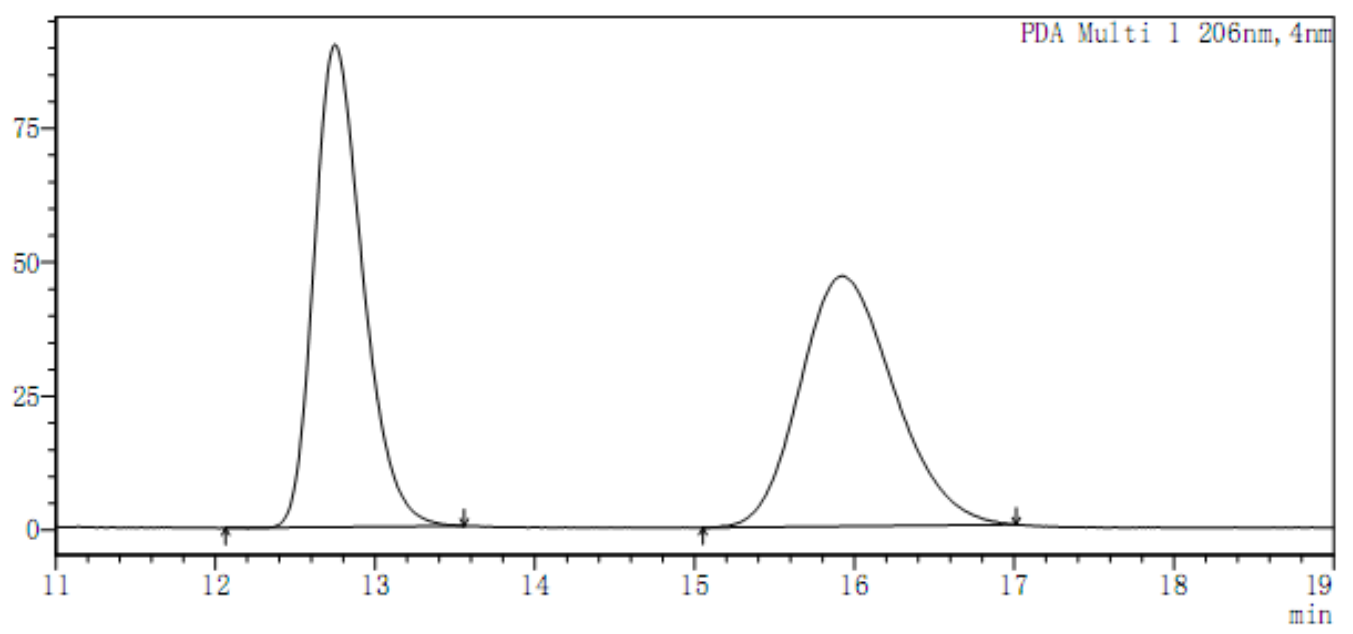

〈Peak Results>

PDA Ch1 206nm

\begin{tabular}{|c|c|c|c|c|}
\hline Index & Time/min & Height/mAU & Quantity/Area & Area \%/\% \\
\hline 1 & 12.748 & 90125 & 1905769 & 49.844 \\
\hline 2 & 15.924 & 46723 & 1917727 & 50.156 \\
\hline
\end{tabular}

〈Chromatogram〉

IIAU

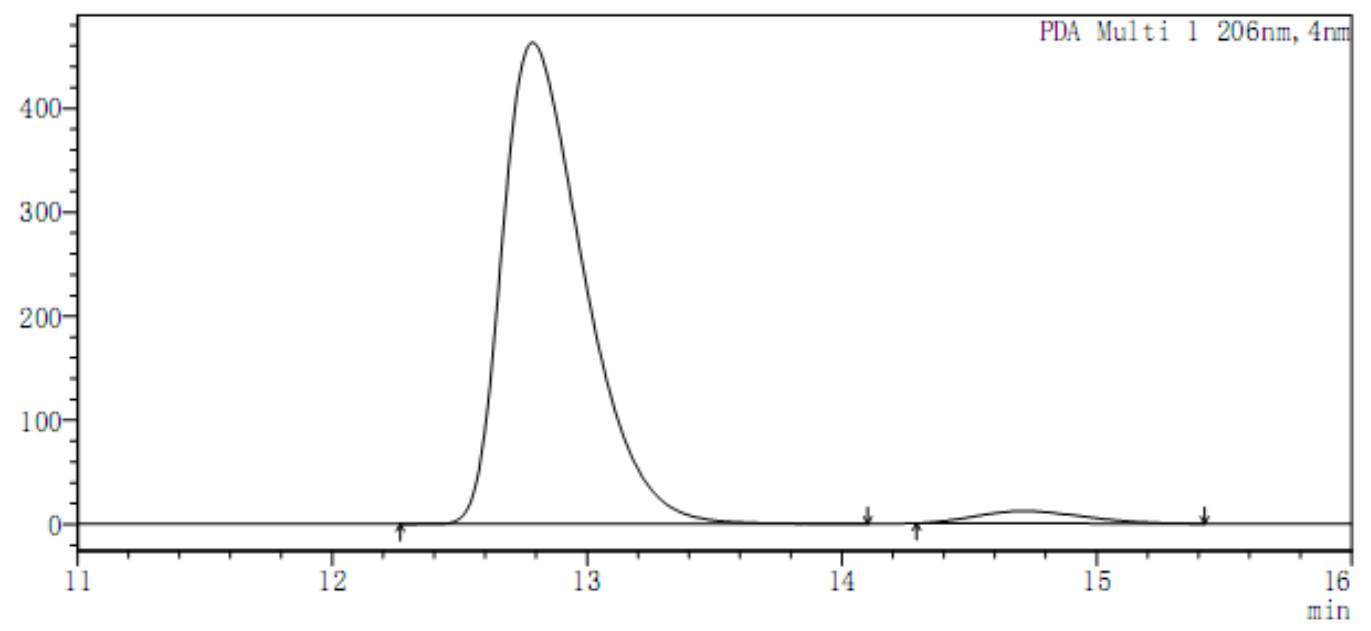

〈Peak Results〉

PDA Ch1 206nm

\begin{tabular}{|c|c|c|c|c|}
\hline Index & Time/min & Height/mAU & Quantity/Area & Area \%/\% \\
\hline 1 & 12.787 & 463150 & 10304310 & 96.743 \\
\hline 2 & 14.713 & 11854 & 346881 & 3.257 \\
\hline
\end{tabular}




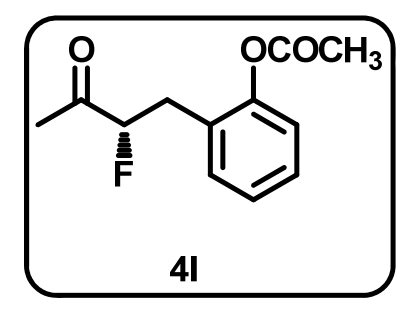

〈Chromatogram〉

mAU

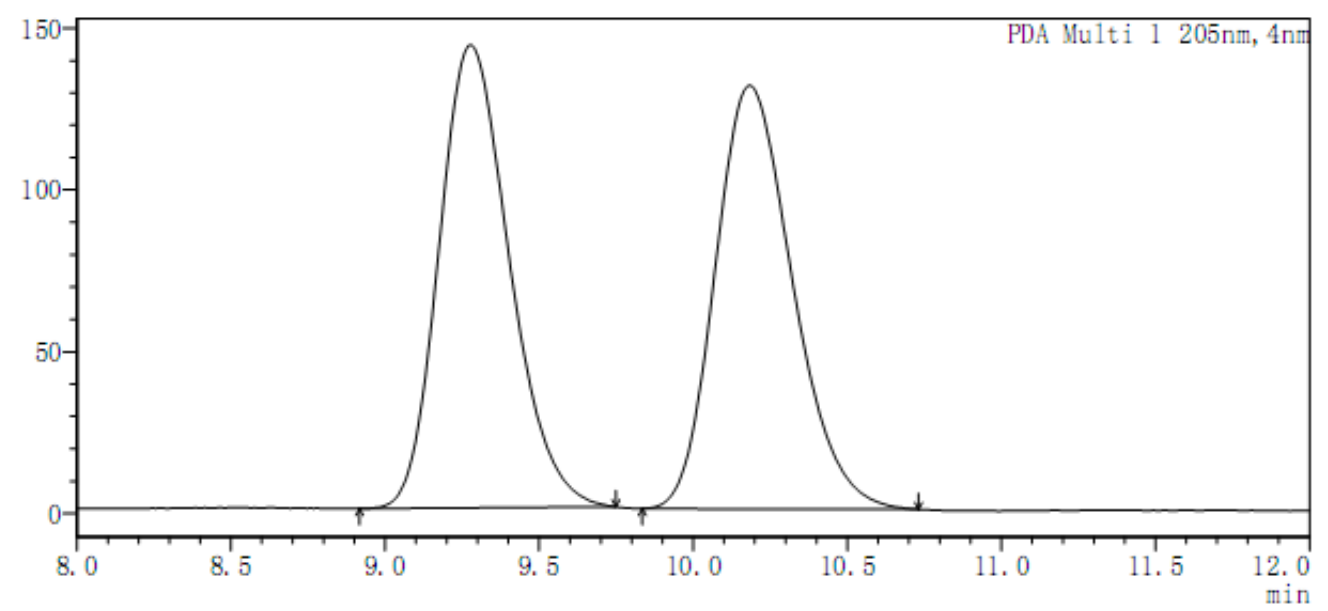

〈Peak Results〉

PDA Chl 205nm

\begin{tabular}{|c|c|c|c|c|}
\hline Index & Time/min & Height/mAU & Quanti ty/Area & Area \%/\% \\
\hline 1 & 9.277 & 143095 & 2272812 & 49.870 \\
\hline 2 & 10.184 & 130868 & 2284683 & 50.130 \\
\hline
\end{tabular}

〈Chromatogram〉

mAU

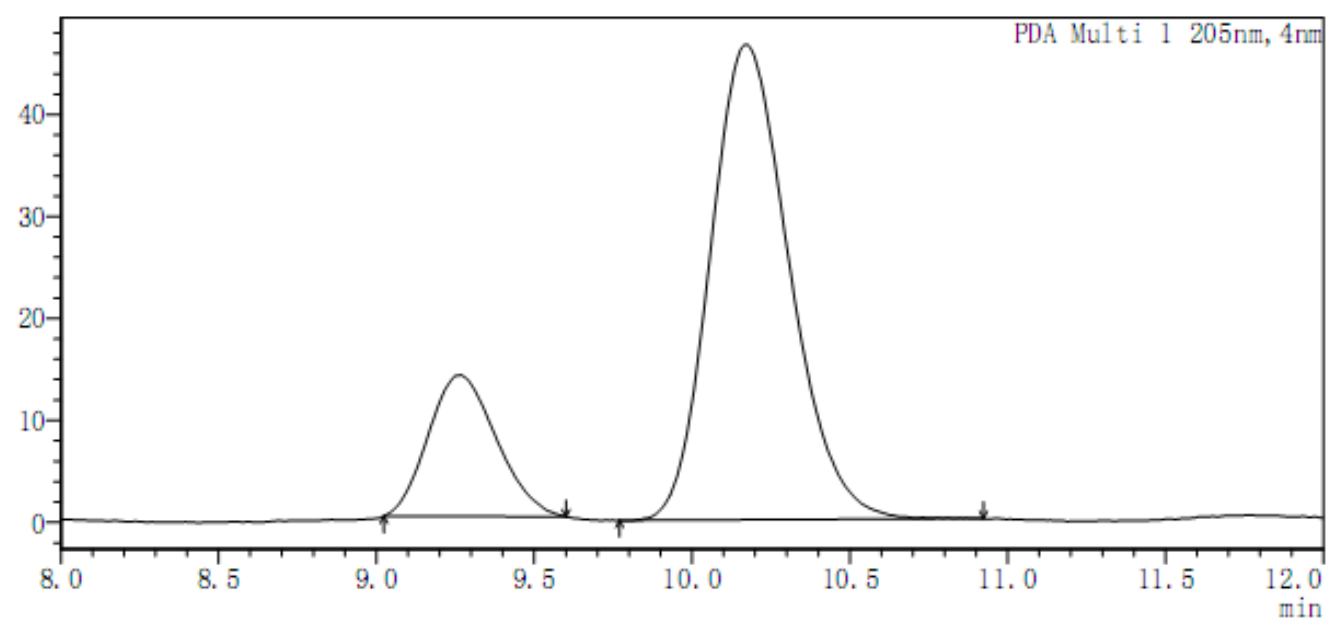

〈Peak Results〉

PDA Chl 205nm

\begin{tabular}{|c|c|c|c|c|}
\hline Index & Time/min & Height/mAU & Quantity/Area & Area \%/\% \\
\hline 1 & 9.265 & 13852 & 207738 & 20.358 \\
\hline 2 & 10.171 & 46601 & 812671 & 79.642 \\
\hline
\end{tabular}




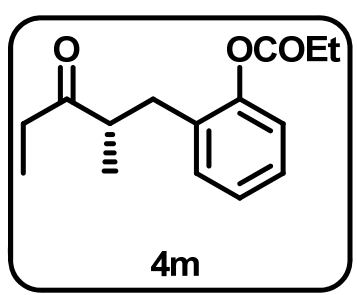

$\langle$ Chromatogram〉

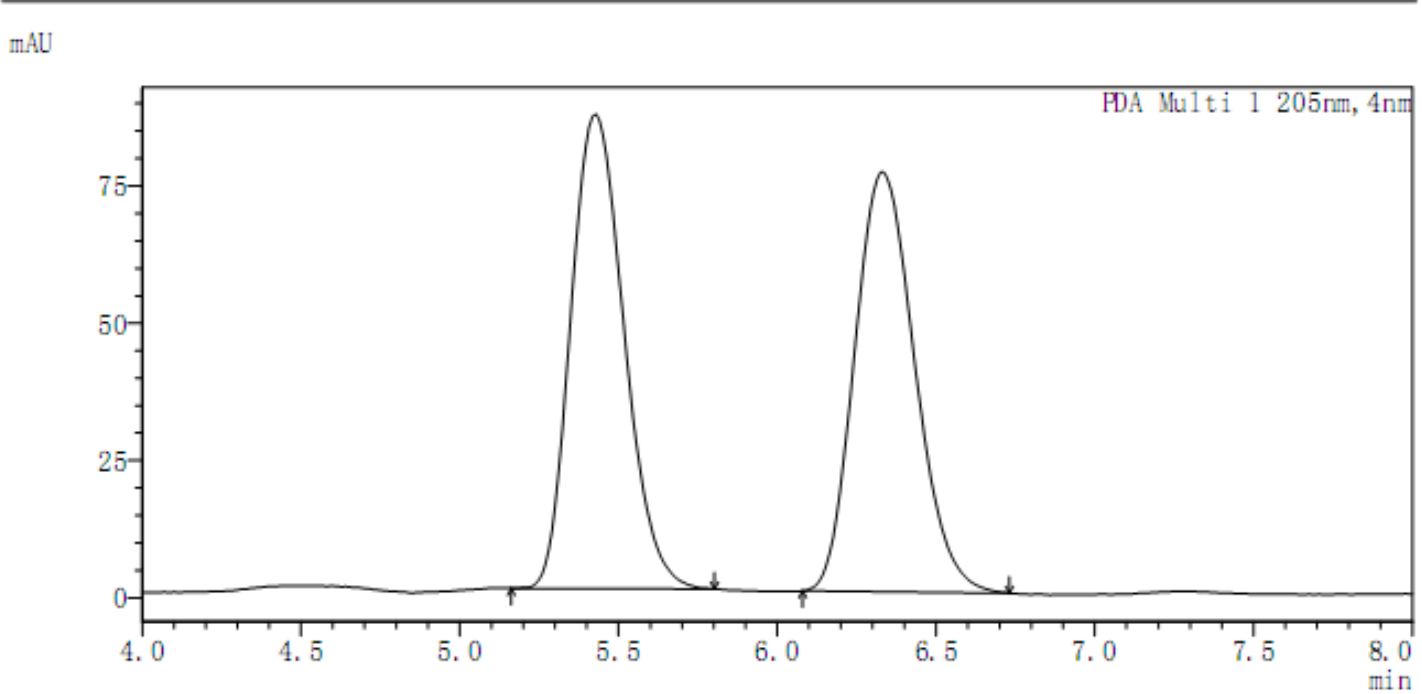

〈Peak Results〉

PDA Chl 205nm

\begin{tabular}{|c|c|c|c|c|}
\hline Index & Time/min & Height/mAU & Quantity/Area & Area \%/\% \\
\hline 1 & 5.427 & 86394 & 1007652 & 50.015 \\
\hline 2 & 6.331 & 76392 & 1007037 & 49.985 \\
\hline
\end{tabular}

$\langle$ Chromatogram〉

$$
\text { mAU }
$$

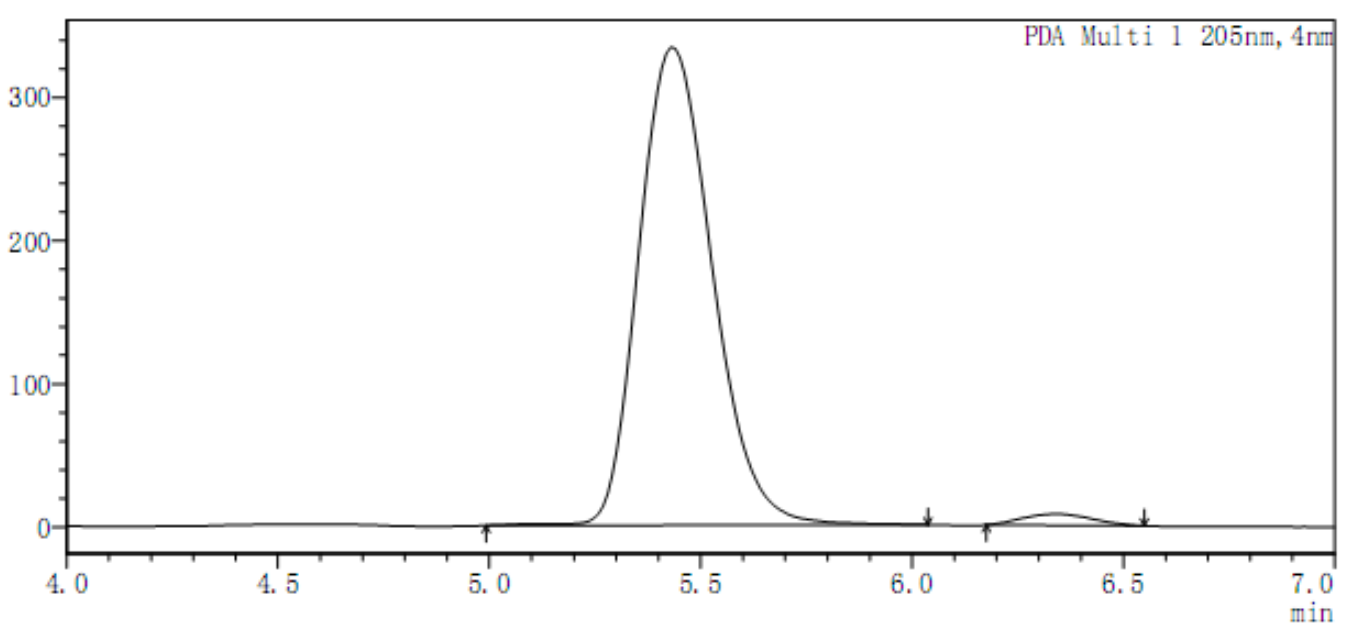

〈Peak Results〉

PDA Chl $205 \mathrm{~nm}$

\begin{tabular}{|c|c|c|c|c|}
\hline Index & Time/min & Height/mAU & Quant ity/Area & Area \%/\% \\
\hline 1 & 5.433 & 333834 & 4029867 & 97.804 \\
\hline 2 & 6.338 & 7864 & 90487 & 2.196 \\
\hline
\end{tabular}




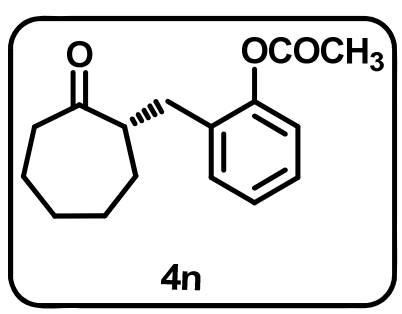

$\langle$ Chromatogram〉

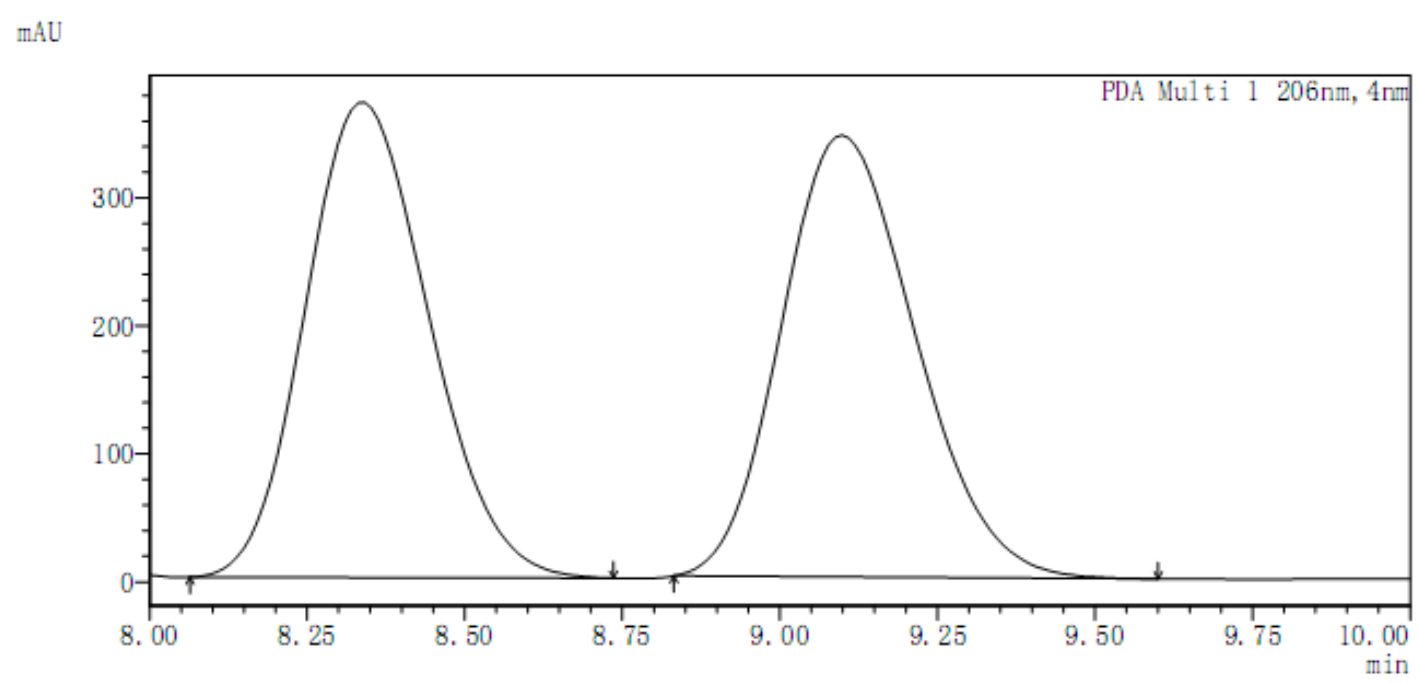

〈Peak Results>

PDA Chl 206nm

\begin{tabular}{|c|c|c|c|c|}
\hline Index & Time/min & Height/mAU & Quantity/Area & Area \%/\% \\
\hline 1 & 8.337 & 371280 & 5064215 & 49.812 \\
\hline 2 & 9.098 & 345160 & 5102395 & 50.188 \\
\hline
\end{tabular}

〈Chromatogram〉

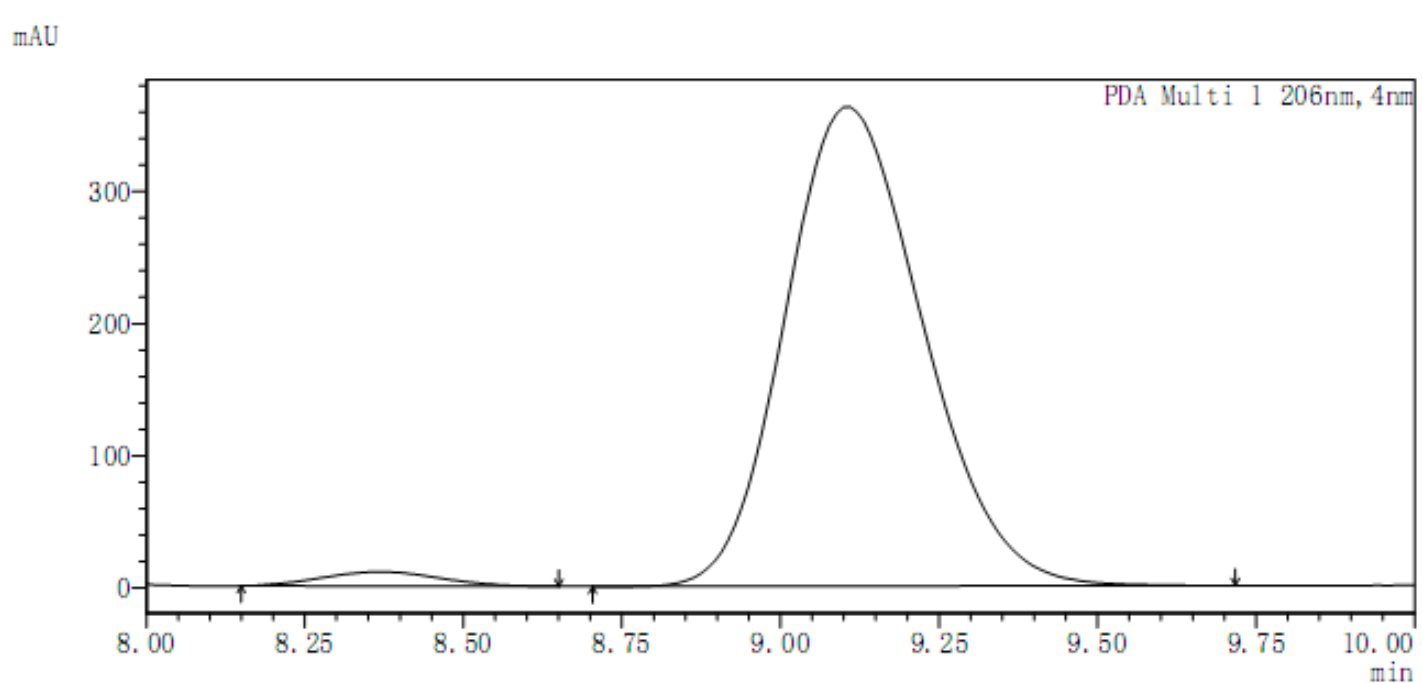

〈Peak Results〉

PDA Ch1 206nm

\begin{tabular}{|c|c|c|c|c|}
\hline Index & Time/min & Height/mAU & Quanti ty/Area & Area \%/\% \\
\hline 1 & 8.369 & 10852 & 140697 & 2.502 \\
\hline 2 & 9.105 & 362639 & 5483515 & 97.498 \\
\hline
\end{tabular}




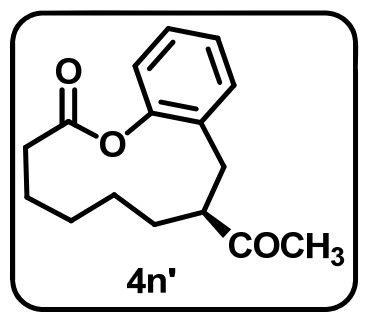

$\langle$ Chromatogram〉

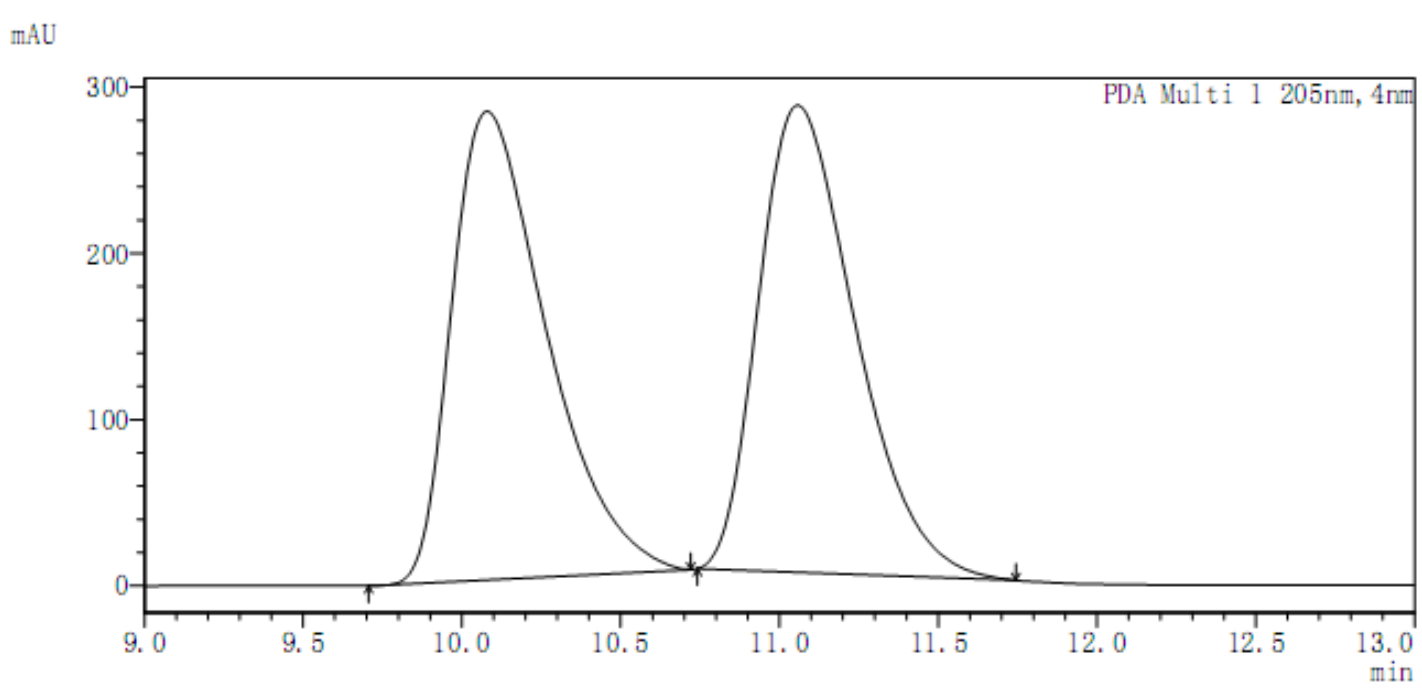

〈Peak Results〉

PDA Ch1 205nm

\begin{tabular}{|c|c|c|c|c|}
\hline Index & Time/min & Height/mAU & Quantity/Area & Area \%/\% \\
\hline 1 & 10.079 & 282079 & 5862855 & 49.634 \\
\hline 2 & 11.057 & 281000 & 5949399 & 50.366 \\
\hline
\end{tabular}

$\langle$ Chromatogram〉

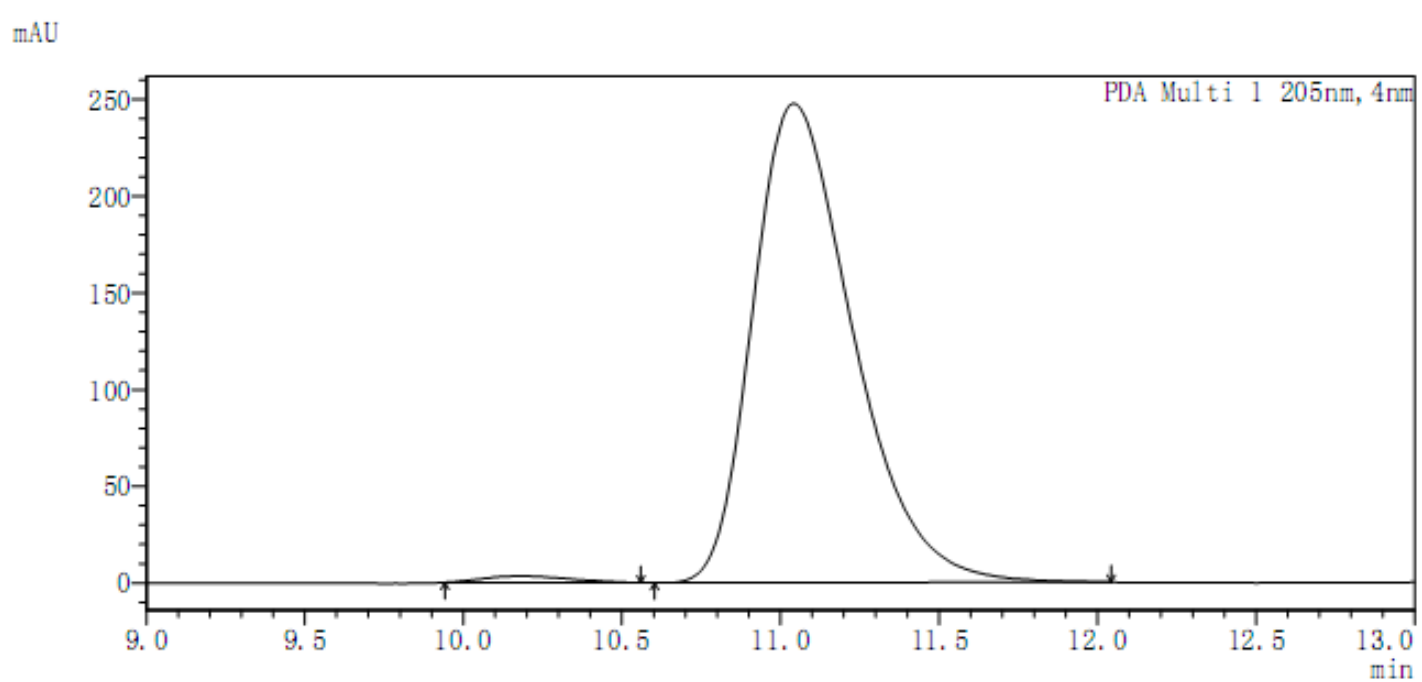

$\langle$ Peak Results〉

PDA Ch1 205nm

\begin{tabular}{|c|c|c|c|c|}
\hline Index & Time/min & Height/mAU & Quant ity/Area & Area \%/\% \\
\hline 1 & 10.184 & 3354 & 63072 & 1.145 \\
\hline 2 & 11.042 & 247692 & 5444367 & 98.855 \\
\hline
\end{tabular}




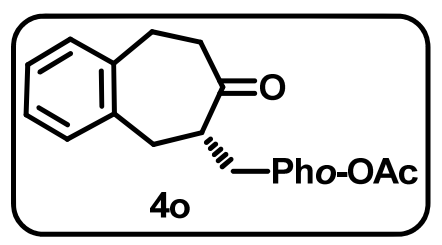

$\langle$ Chromatogram〉

$\mathrm{MAU}$

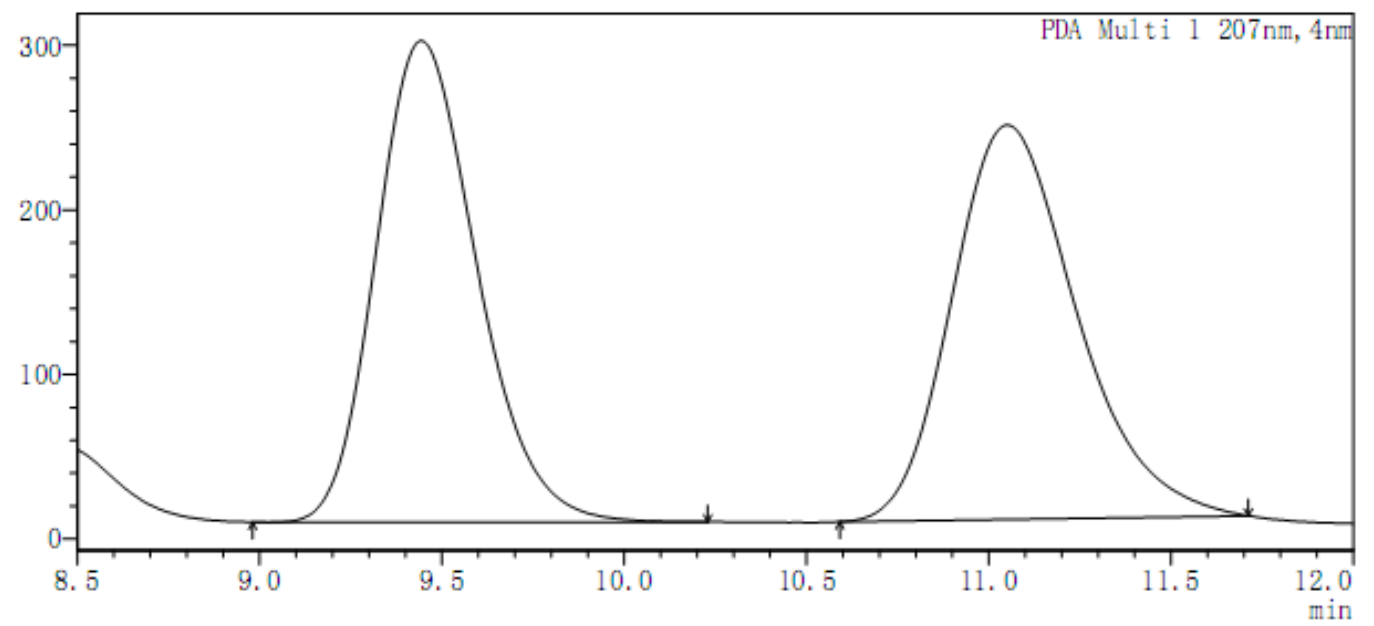

〈Peak Results〉

PDA Chl 207nm

\begin{tabular}{|c|c|c|c|c|}
\hline Index & Time/min & Height/mAU & Quantity/Area & Area \%/\% \\
\hline 1 & 9.444 & 292799 & 5622010 & 49.663 \\
\hline 2 & 11.051 & 239905 & 5698413 & 50.337 \\
\hline
\end{tabular}

〈Chromatogram〉

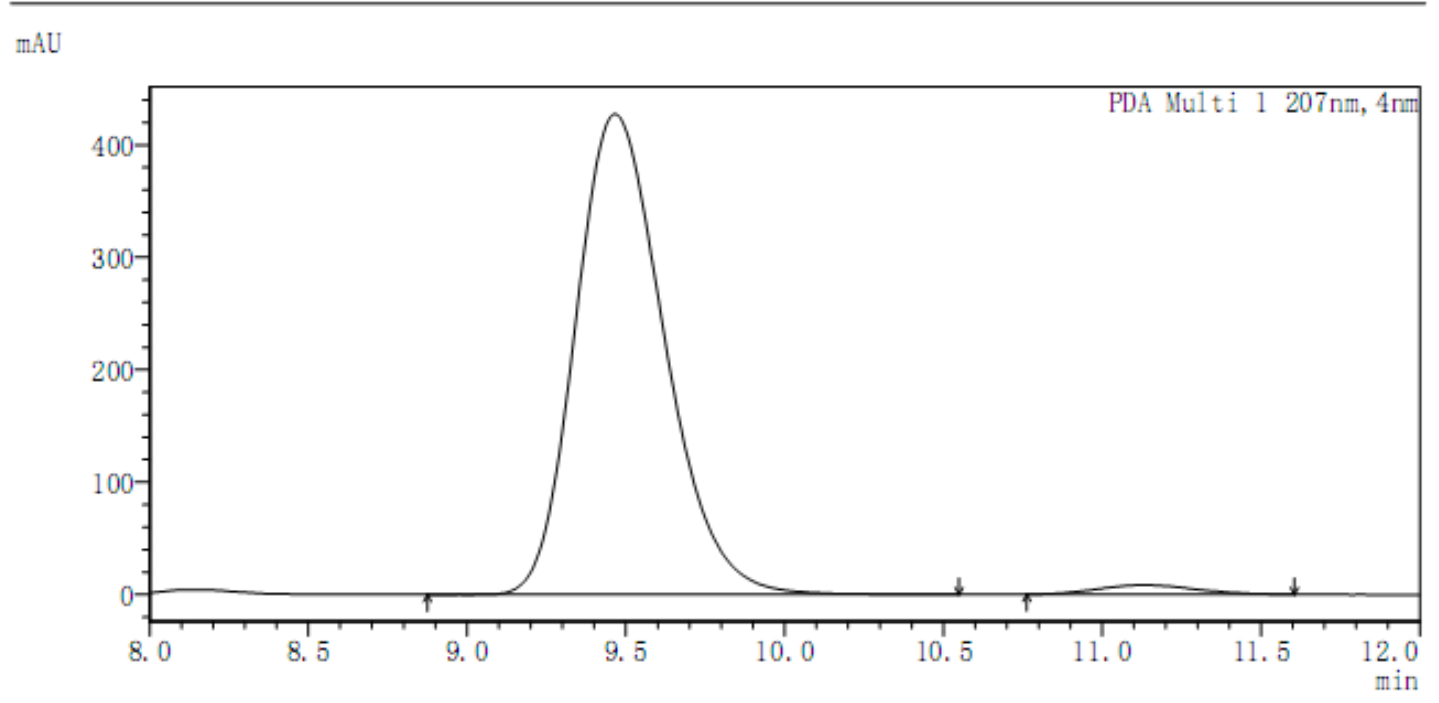

〈Peak Results〉

PDA Chl 207nm

\begin{tabular}{|c|c|c|c|c|}
\hline Index & Time/min & Height/mAU & Quantity/Area & Area \%/\% \\
\hline 1 & 9.465 & 427869 & 8341515 & 97.869 \\
\hline 2 & 11.133 & 8339 & 181647 & 2.131 \\
\hline
\end{tabular}




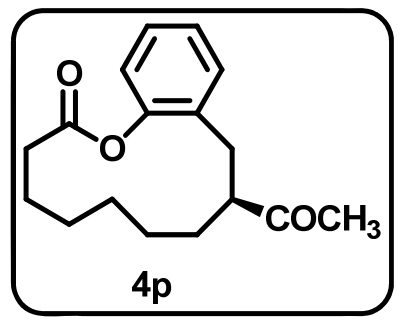

$\langle$ Chromatogram〉

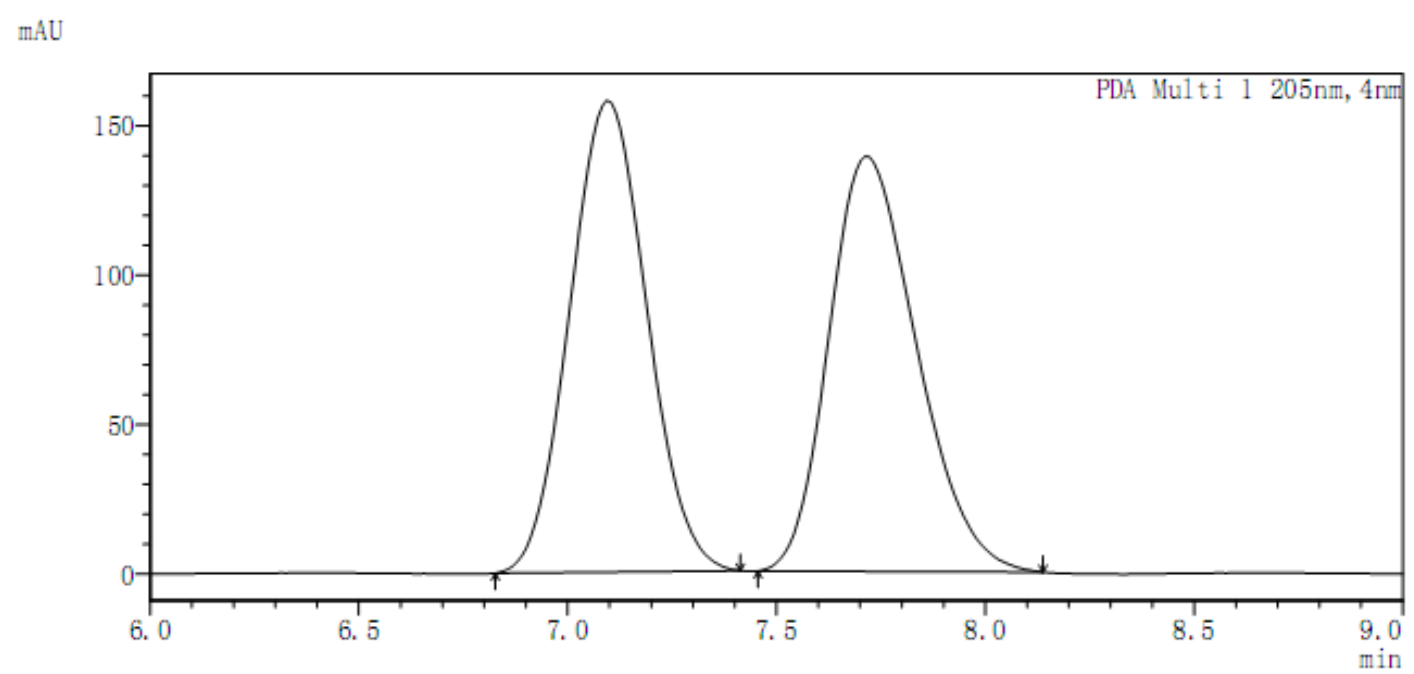

$\langle$ Peak Results〉

PDA Chl 205nm

\begin{tabular}{|c|c|c|c|c|}
\hline Index & Time/min & Height/mAU & Quantity/Area & Area \%/\% \\
\hline 1 & 7.096 & 157921 & 2029266 & 50.003 \\
\hline 2 & 7.716 & 138945 & 2029028 & 49.997 \\
\hline
\end{tabular}

〈Chromatogram〉

mAU

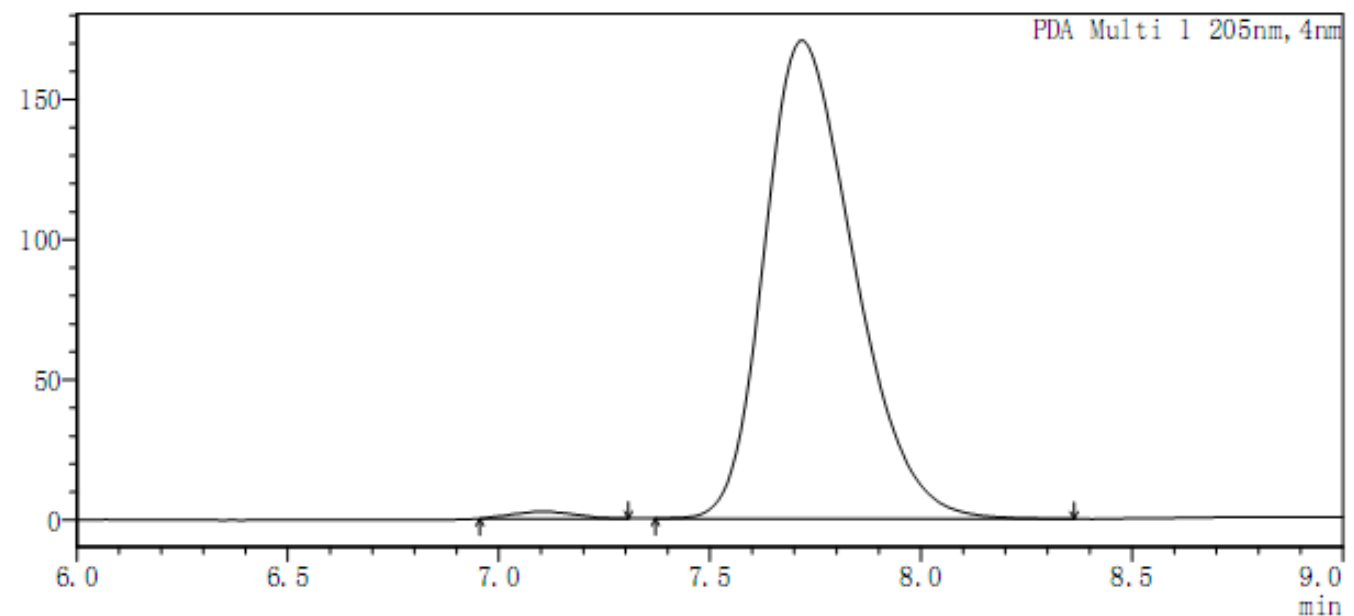

〈Peak Results〉

PDA Chl 205nm

\begin{tabular}{|c|c|c|c|c|}
\hline Index & Time/min & Height/mAU & Quantity/Area & Area \%/\% \\
\hline 1 & 7.104 & 2438 & 25705 & 1.010 \\
\hline 2 & 7.717 & 170343 & 2518049 & 98.990 \\
\hline
\end{tabular}




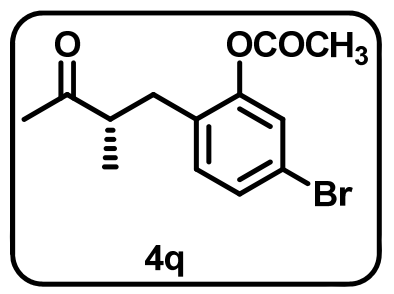

$\langle$ Chromatogram〉

MAU

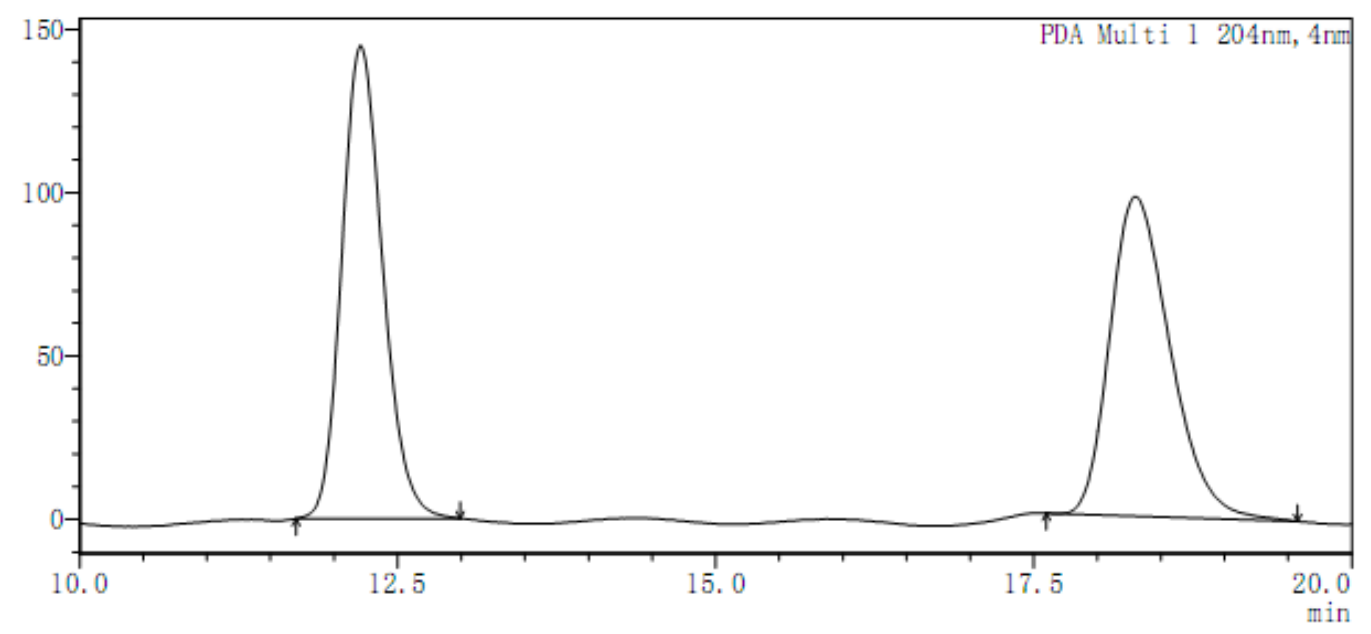

〈Peak Results〉

PDA Ch1 204nm

\begin{tabular}{|c|c|c|c|c|}
\hline Index & Time/min & Height/mAU & Quantity/Area & Area \%/\% \\
\hline 1 & 12.208 & 144729 & 3235759 & 50.099 \\
\hline 2 & 18.296 & 97815 & 3222988 & 49.901 \\
\hline
\end{tabular}

〈Chromatogram〉

mAU

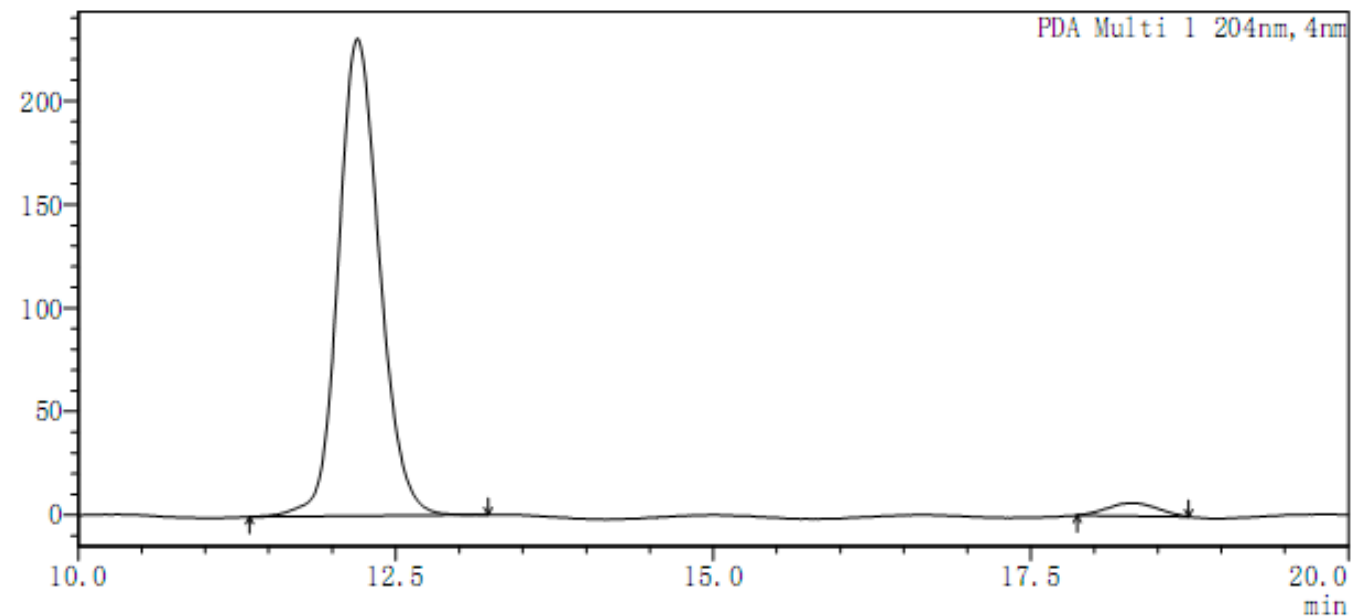

〈Peak Results>

PDA Ch1 204nm

\begin{tabular}{|c|c|c|c|c|}
\hline Index & Time/min & Height/mAU & Quantity/Area & Area \%/\% \\
\hline 1 & 12.199 & 230431 & 5209167 & 96.812 \\
\hline 2 & 18.284 & 6236 & 171511 & 3.188 \\
\hline
\end{tabular}




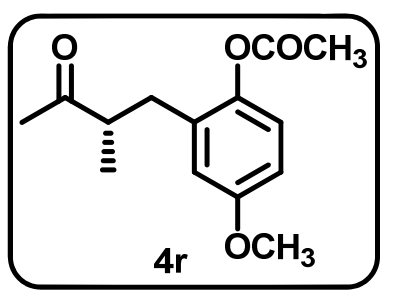

$\langle$ Chromatogram〉

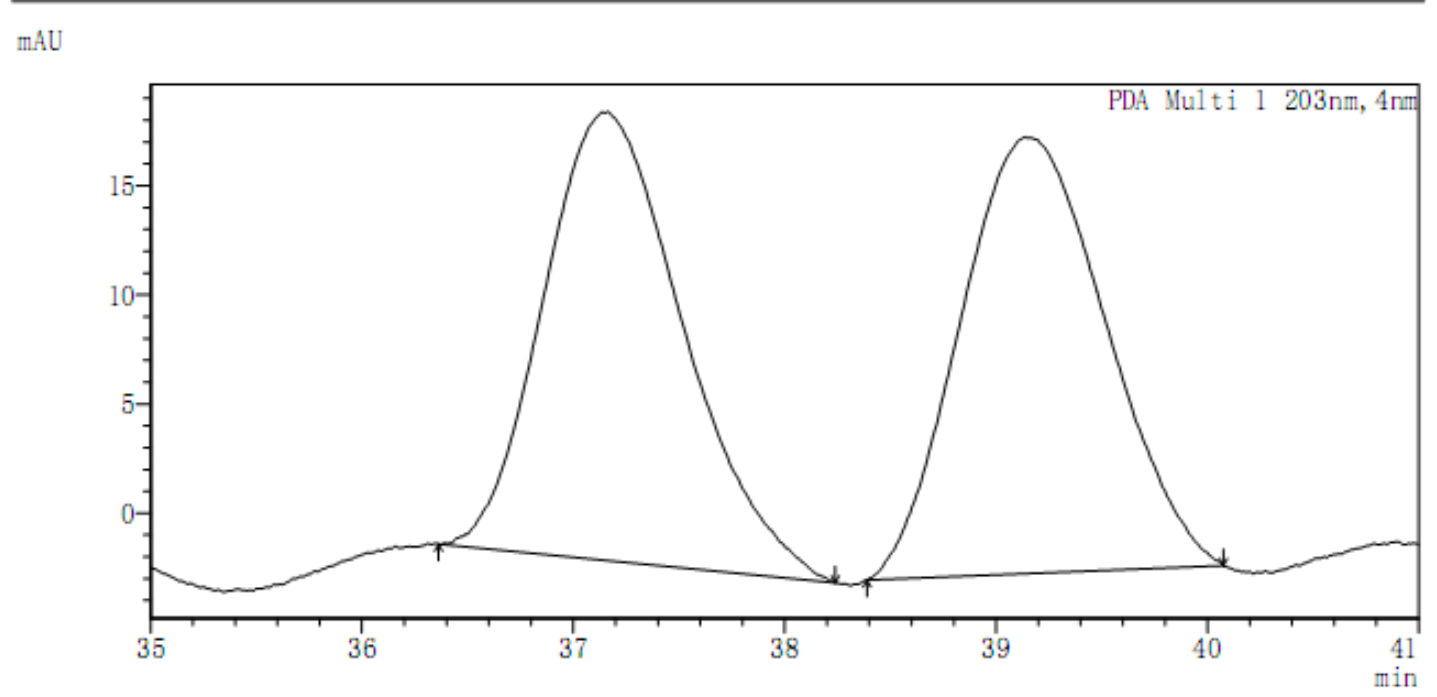

〈Peak Results〉

PDA Chl 203nm

\begin{tabular}{|c|c|c|c|c|}
\hline Index & Time/min & Height/mAU & Quanti ty/Area & Area \%/\% \\
\hline 1 & 37.163 & 20571 & 942363 & 49.683 \\
\hline 2 & 39.136 & 20020 & 954380 & 50.317 \\
\hline
\end{tabular}

〈Chromatogram〉

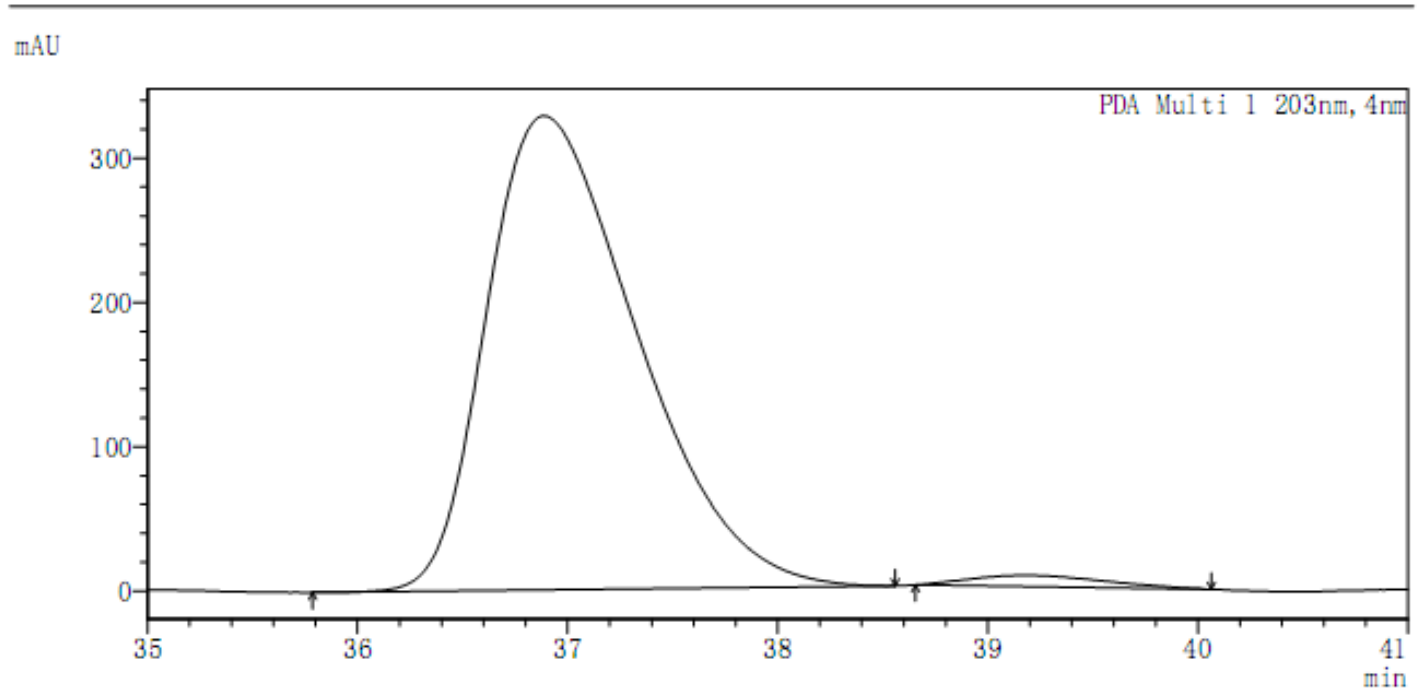

〈Peak Results〉

PDA Ch1 203nm

\begin{tabular}{|c|c|c|c|c|}
\hline Index & Time/min & Height/mAU & Quantity/Area & Area \%/\% \\
\hline 1 & 36.889 & 328545 & 16389651 & 97.915 \\
\hline 2 & 39.204 & 7823 & 348956 & 2.085 \\
\hline
\end{tabular}




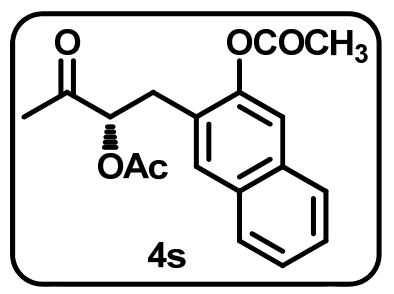

$\langle$ Chromatogram〉

$\mathrm{mAU}$

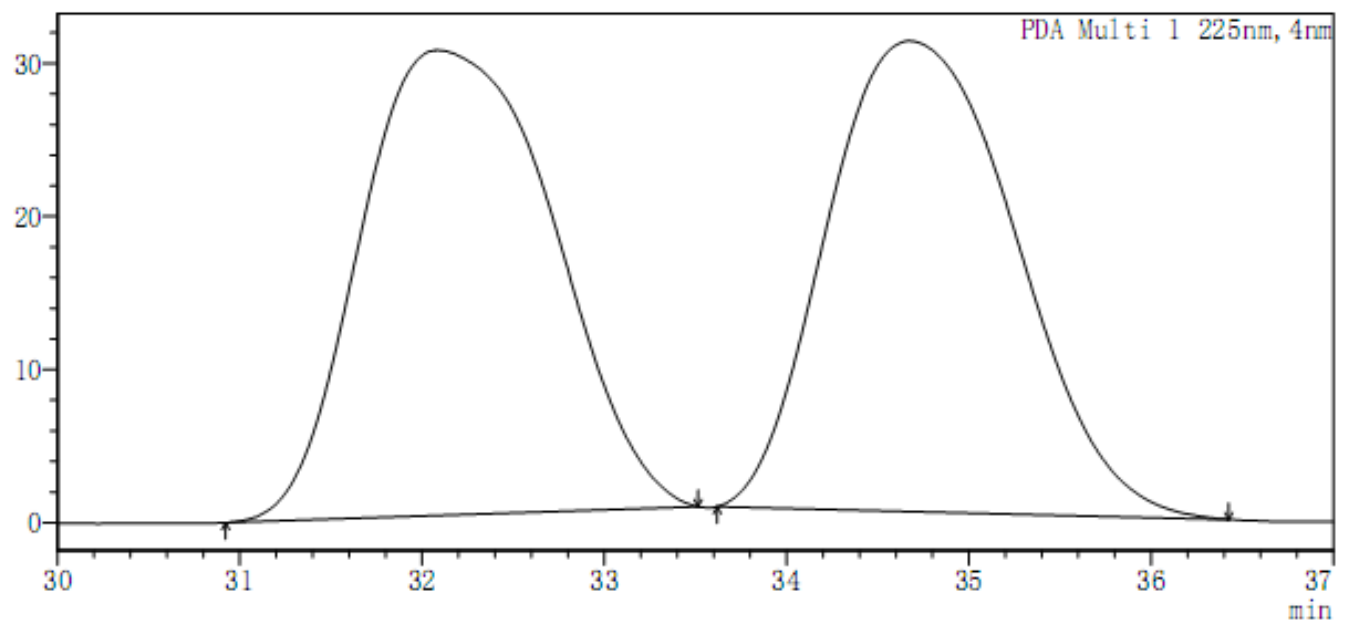

〈Peak Results>

PDA Ch1 225nm

\begin{tabular}{|c|c|c|c|c|}
\hline Index & Time/min & Height/mAU & Quanti ty/Area & Area \%/\% \\
\hline 1 & 32.085 & 30376 & 2232080 & 50.200 \\
\hline 2 & 34.671 & 30730 & 2214260 & 49.800 \\
\hline
\end{tabular}

〈Chromatogram〉

MAU

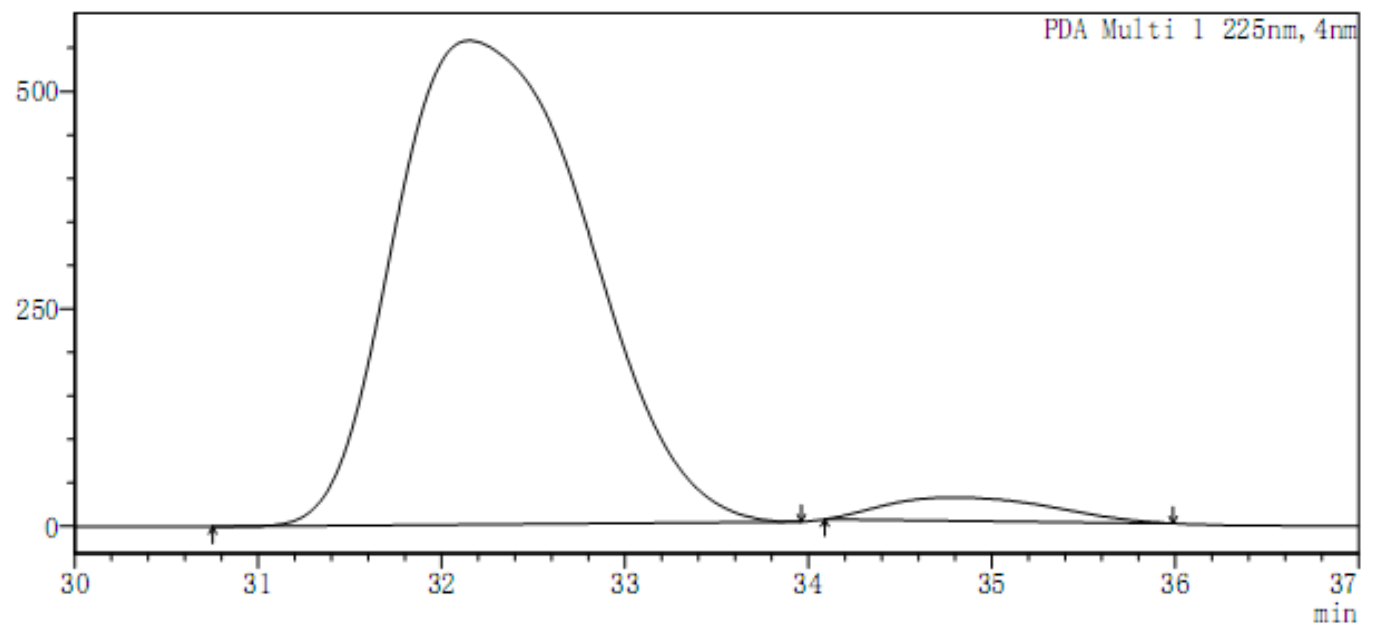

〈Peak Results〉

PDA Chl 225nm.

\begin{tabular}{|c|c|c|c|c|}
\hline Index & Time/min & Height/mAU & Quantity/Area & Area \%/\% \\
\hline 1 & 32.152 & 556225 & 40593863 & 95.952 \\
\hline 2 & 34.792 & 26842 & 1712566 & 4.048 \\
\hline
\end{tabular}




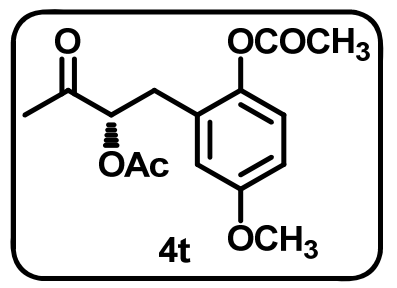

$\langle$ Chromatogram〉

mAU

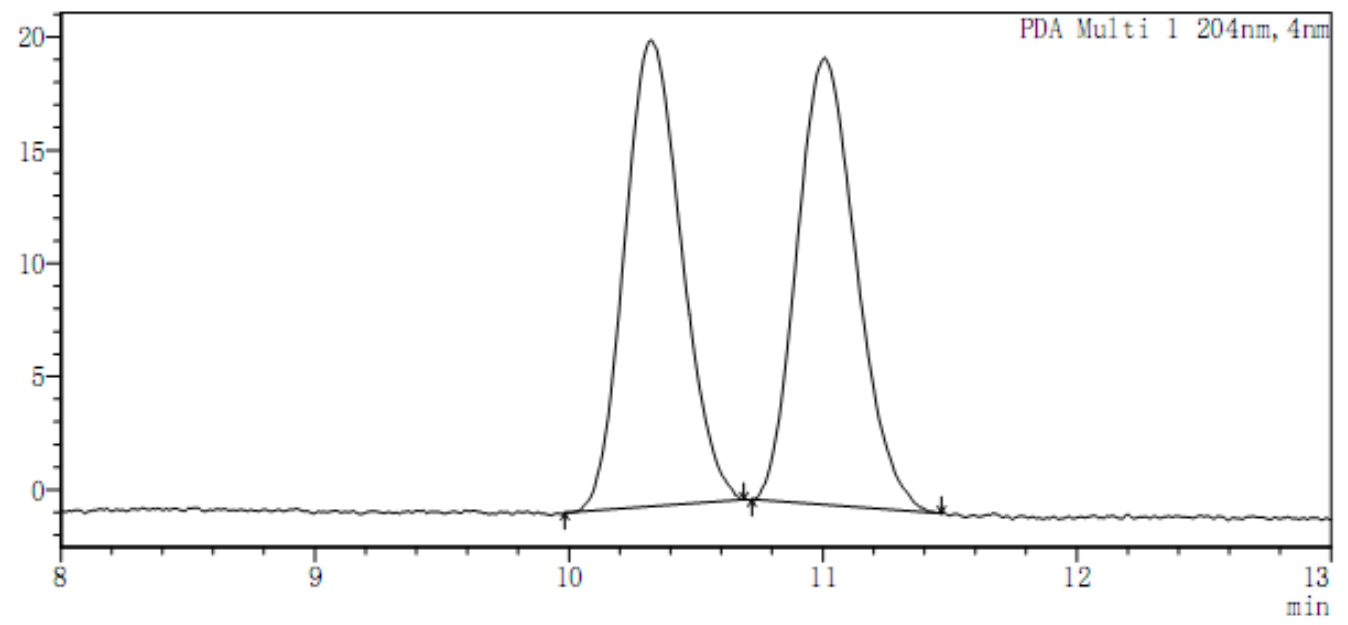

〈Peak Results>

PDA Ch1 204nm

\begin{tabular}{|c|c|c|c|c|}
\hline Index & Time/min & Height/mAU & Quantity/Area & Area \%/\% \\
\hline 1 & 10.323 & 20609 & 321425 & 49.973 \\
\hline 2 & 11.006 & 19754 & 321773 & 50.027 \\
\hline
\end{tabular}

$\langle$ Chromatogram〉

MAU

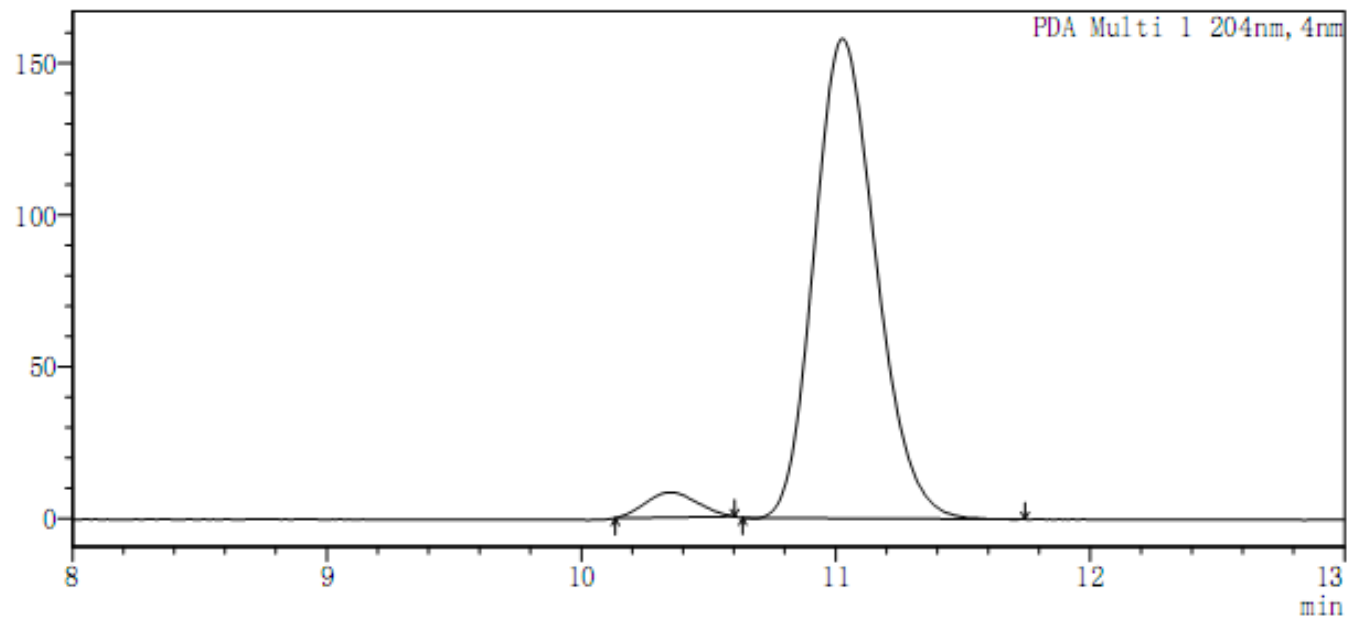

〈Peak Results>

PDA Chl 204nm

\begin{tabular}{|c|c|c|c|c|}
\hline Index & Time/min & Height/mAU & Quant ity/Area & Area \%/\% \\
\hline 1 & 10.349 & 8220 & 116976 & 4.203 \\
\hline 2 & 11.026 & 158150 & 2666327 & 95.797 \\
\hline
\end{tabular}




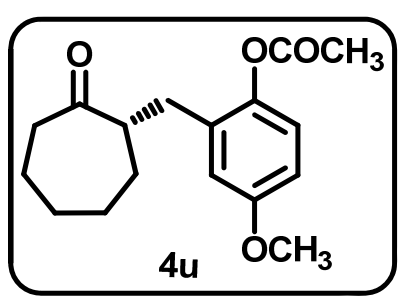

$\langle$ Chromatogram〉

$\mathrm{mAU}$

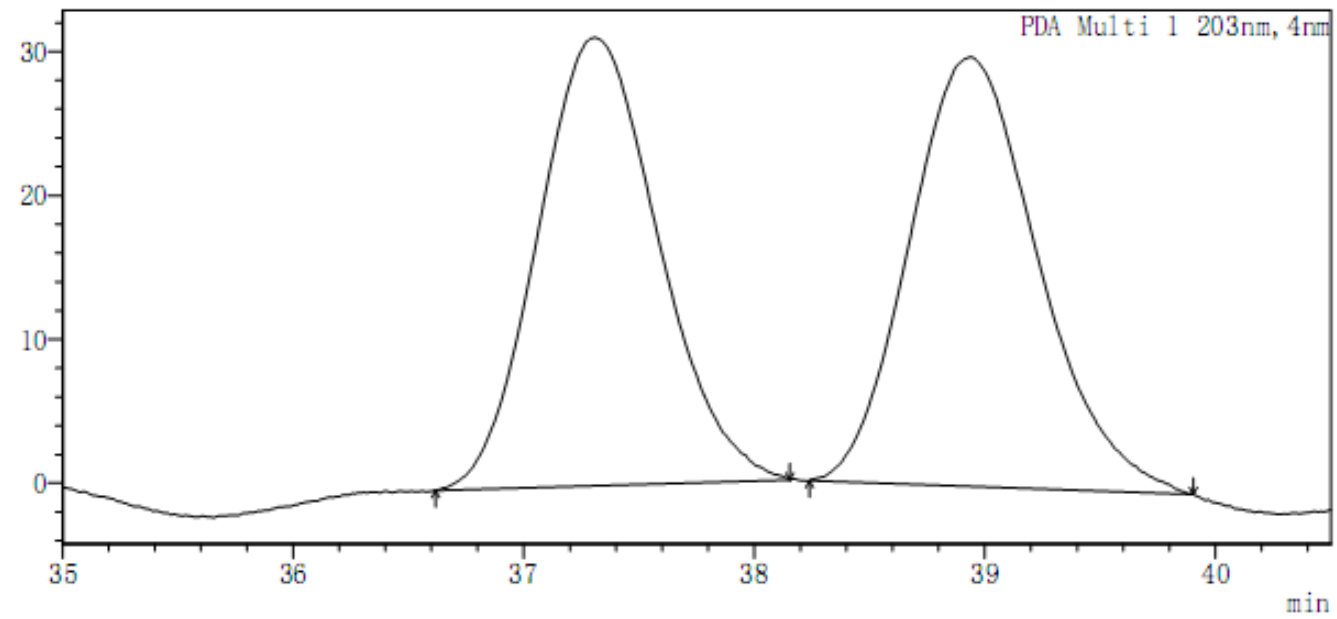

〈Peak Results>

PDA Ch1 203nm

\begin{tabular}{|c|c|c|c|c|}
\hline Index & Time/min & Height/mAU & Quantity/Area & Area \%/\% \\
\hline 1 & 37.310 & 31156 & 1132304 & 49.449 \\
\hline
\end{tabular}

\begin{tabular}{|c|c|c|c|c|}
\hline 1 & 37.310 & 31156 & 1132304 & 49.449 \\
\hline 2 & 38.937 & 29840 & 1157518 & 50.551 \\
\hline
\end{tabular}

$\langle$ Chromatogram〉

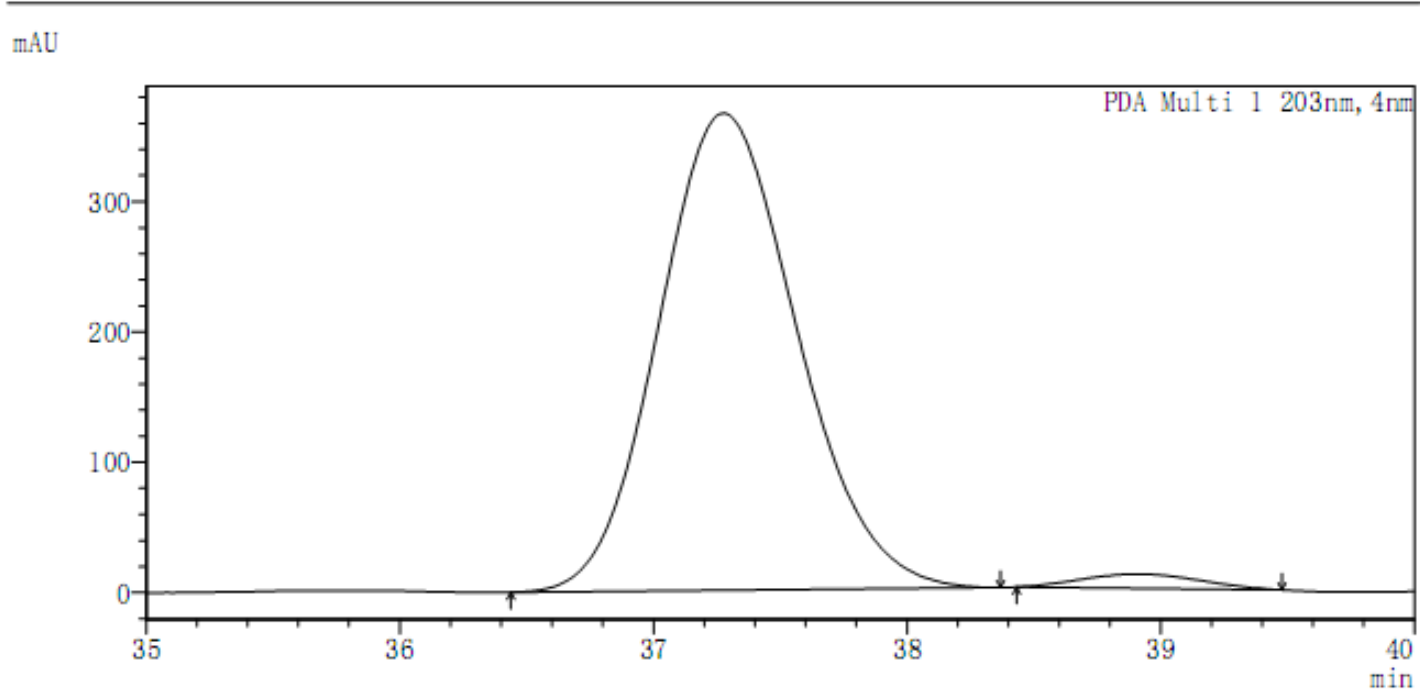

$\langle$ Peak Results>

PDA Ch1 203nm

\begin{tabular}{|c|c|c|c|c|}
\hline Index & Time/min & Height/mAU & Quantity/Area & Area \%/\% \\
\hline 1 & 37.277 & 365926 & 13850964 & 97.492 \\
\hline 2 & 38.907 & 10918 & 356365 & 2.508 \\
\hline
\end{tabular}




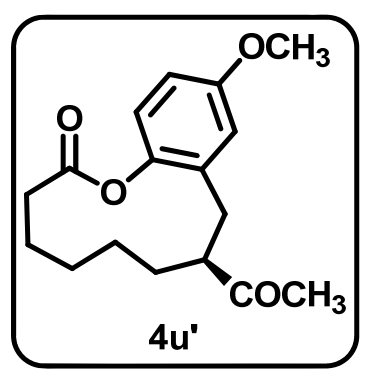

$\langle$ Chromatogram〉

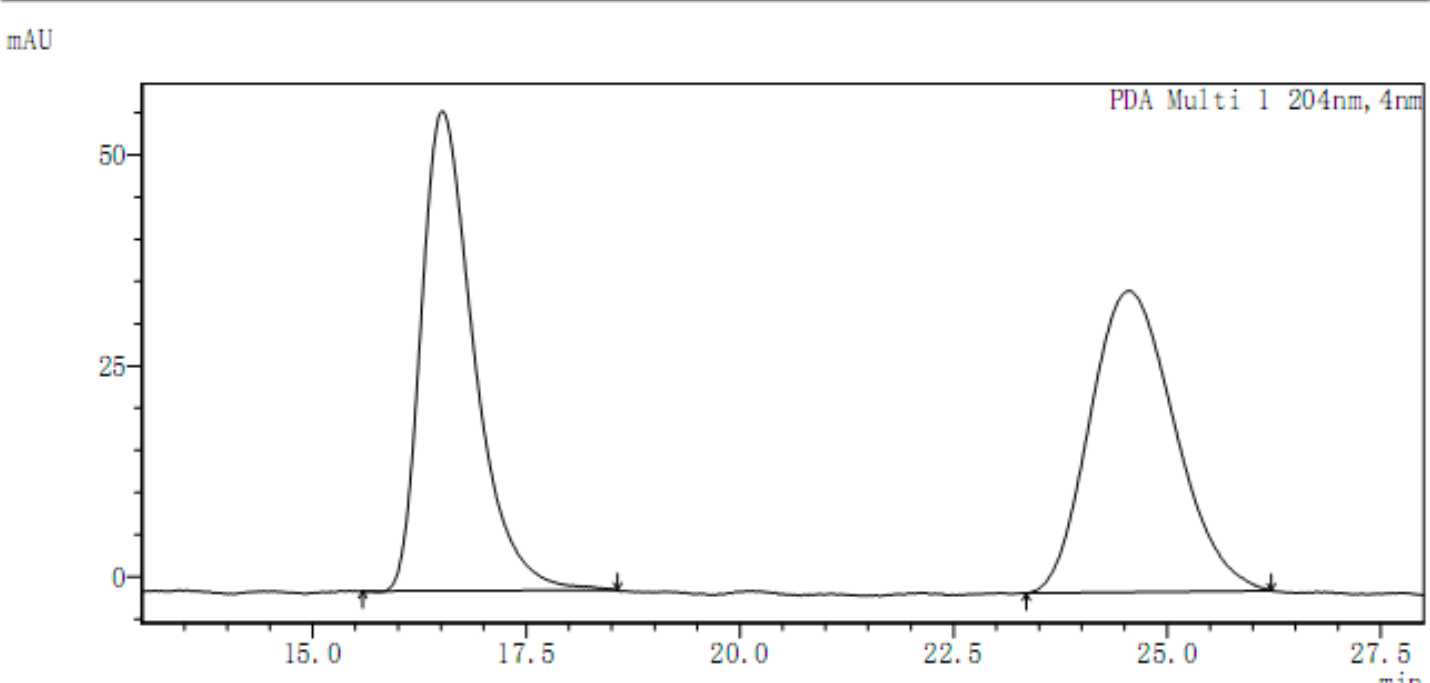

〈Peak Results>

PDA Ch1 204nm

\begin{tabular}{|c|c|c|c|c|}
\hline Index & Time/min & Height/mAU & Quantity/Area & Area \%/\% \\
\hline 1 & 16.518 & 56840 & 2433573 & 49.722 \\
\hline 2 & 24.551 & 35695 & 2460832 & 50.278 \\
\hline
\end{tabular}

〈Chromatogram〉

mAU

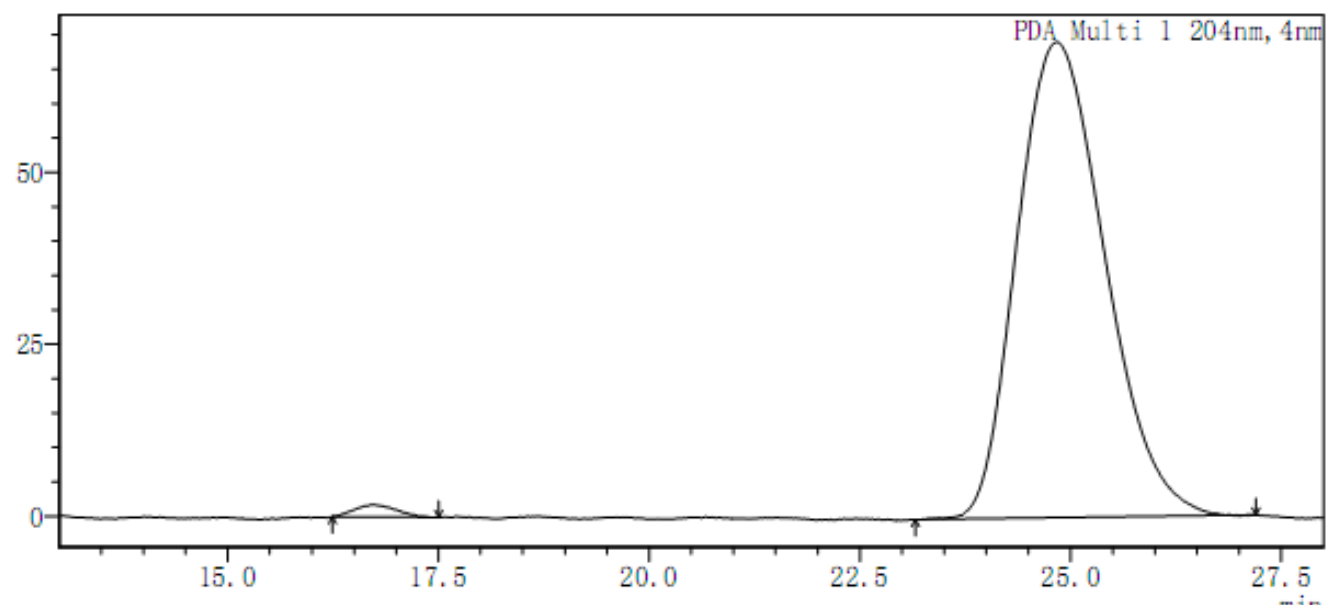

〈Peak Results>

PDA Ch1 204nm

\begin{tabular}{|c|c|c|c|c|}
\hline Index & Time/min & Height/mAU & Quant ity/Area & Area \%/\% \\
\hline 1 & 16.718 & 1791 & 61836 & 1.219 \\
\hline 2 & 24.842 & 69187 & 5009941 & 98.781 \\
\hline
\end{tabular}




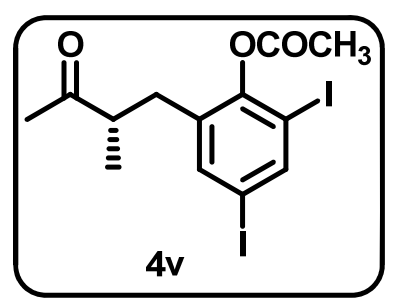

$\langle$ Chromatogram〉

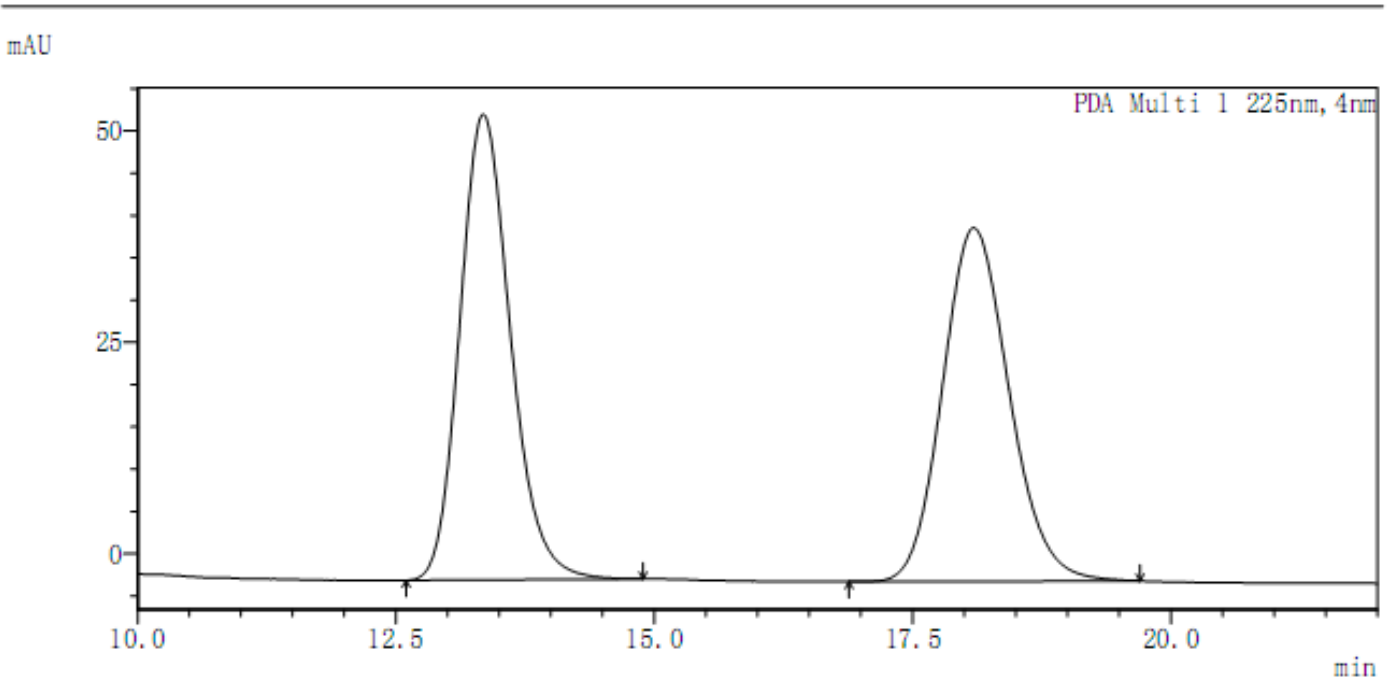

〈Peak Results〉

PDA Chl 225nm

\begin{tabular}{|c|c|c|c|c|}
\hline Index & Time/min & Height/mAU & Quantity/Area & Area \%/\% \\
\hline 1 & 13.345 & 55057 & 1881201 & 50.051 \\
\hline 2 & 18.092 & 41813 & 1877373 & 49.949 \\
\hline
\end{tabular}

$\langle$ Chromatogram〉

mAU

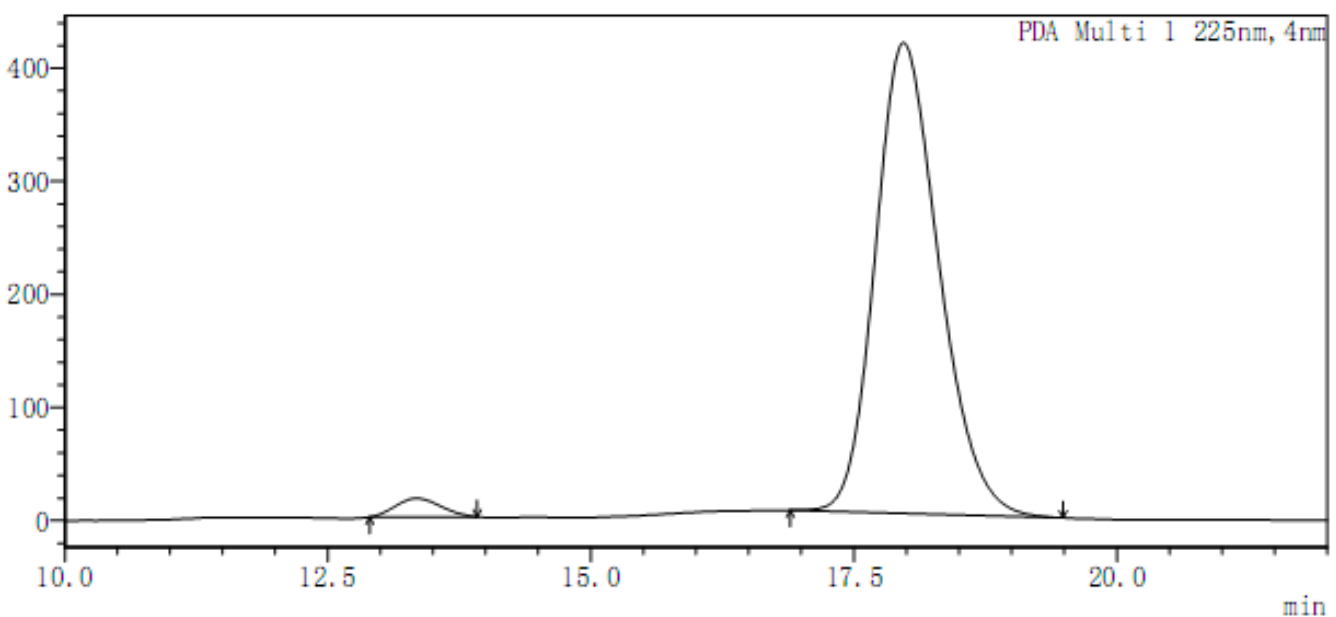

〈Peak Results>

PDA Ch1 225nm

\begin{tabular}{|c|c|c|c|c|}
\hline Index & Time/min & Height/mAU & Quant ity/Area & Area \%/\% \\
\hline 1 & 13.347 & 16349 & 490812 & 2.717 \\
\hline 2 & 17.972 & 416294 & 17570907 & 97.283 \\
\hline
\end{tabular}




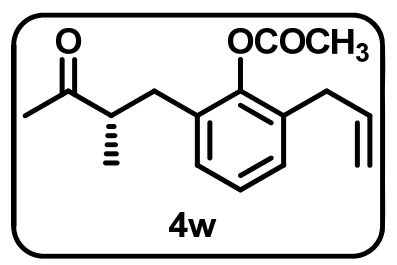

$\langle$ Chromatogram〉

mAU

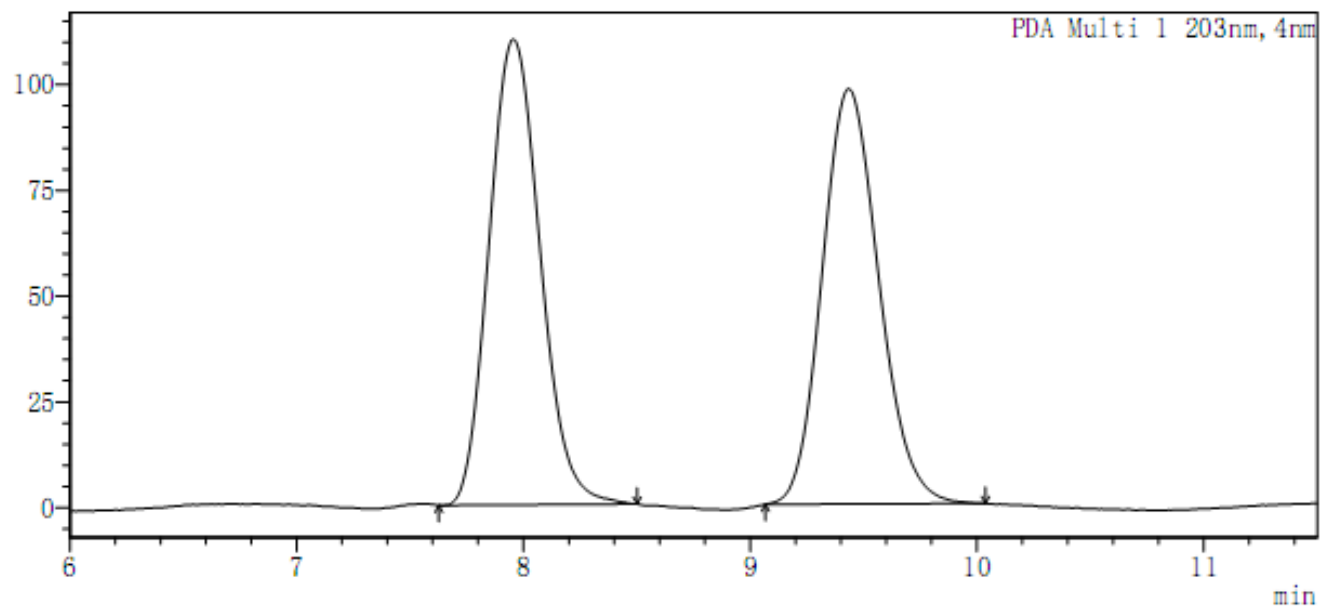

〈Peak Results>

PDA Ch1 203nm

\begin{tabular}{|c|c|c|c|c|}
\hline Index & Time/min & Height/mAU & Quantity/Area & Area \%/\% \\
\hline 1 & 7.956 & 110054 & 1728031 & 50.327 \\
\hline 2 & 9.434 & 98084 & 1705555 & 49.673 \\
\hline
\end{tabular}

〈Chromatogram〉

mAU

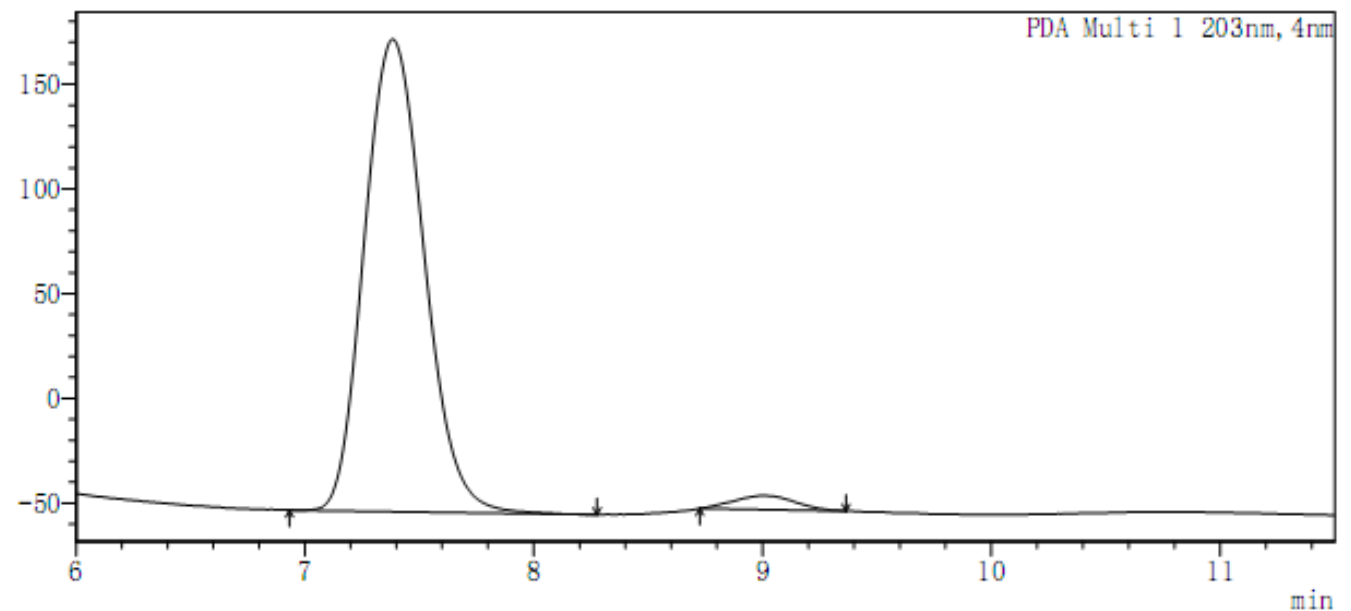

〈Peak Results〉

PDA Ch1 203nm

\begin{tabular}{|c|c|c|c|c|}
\hline Index & Time/min & Height/mAU & Quantity/Area & Area \%/\% \\
\hline 1 & 7.384 & 225772 & 4092905 & 97.051 \\
\hline 2 & 9.004 & 6635 & 124352 & 2.949 \\
\hline
\end{tabular}




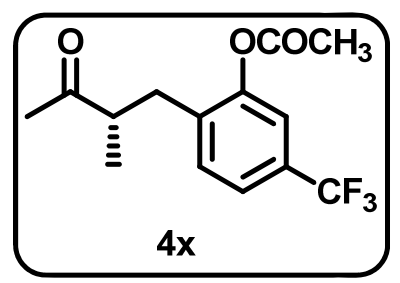

$\langle$ Chromatogram〉

MAL

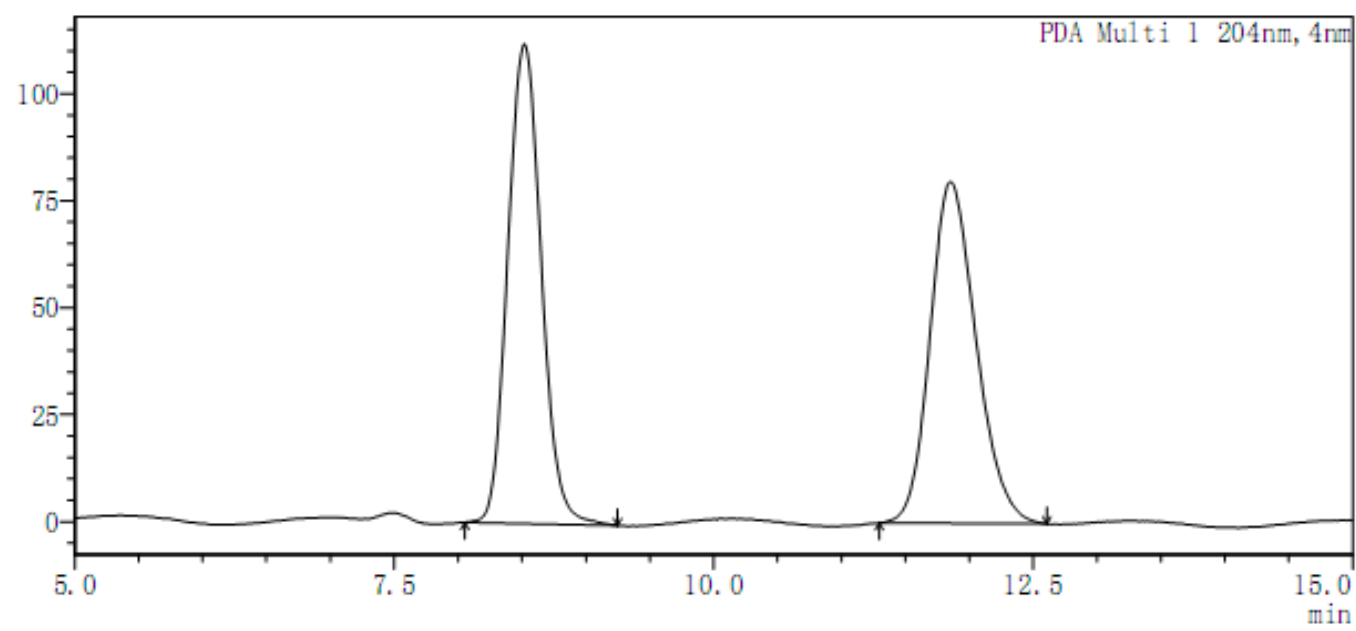

〈Peak Results〉

PDA Chl 204nm

\begin{tabular}{|c|c|c|c|c|}
\hline Index & Time/min & Height/mAU & Quantity/Area & Area \%/\% \\
\hline 1 & 8.518 & 112032 & 2044082 & 50.545 \\
\hline 2 & 11.854 & 79736 & 2000037 & 49.455 \\
\hline
\end{tabular}

〈Chromatogram〉

IIAU

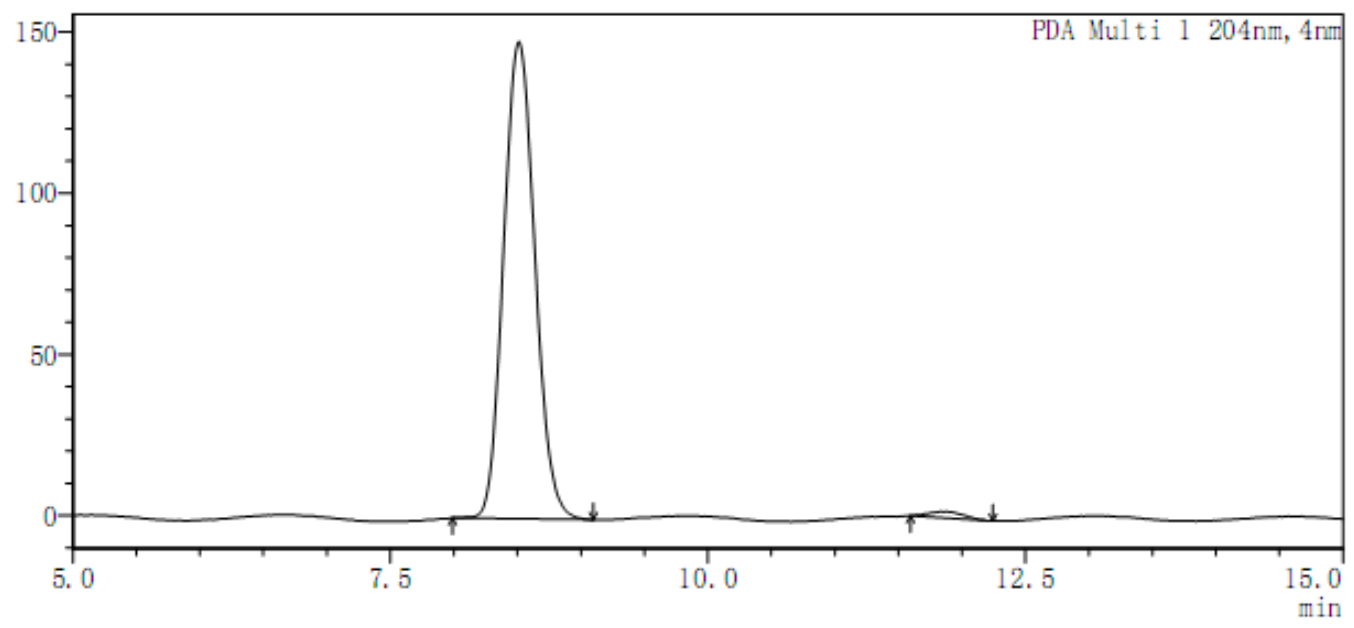

$\langle$ Peak Results〉

PDA Chl 204nm

PDA Chl $204 \mathrm{~nm}$
\begin{tabular}{|c|c|c|c|c|}
\hline Index & Time/min & Height/mAU & Quantity/Area & Area $\% / \%$ \\
\hline 1 & 8.512 & 148097 & 2541464 & 98.582 \\
\hline 2 & 11.833 & 1882 & 36564 & 1.418 \\
\hline
\end{tabular}




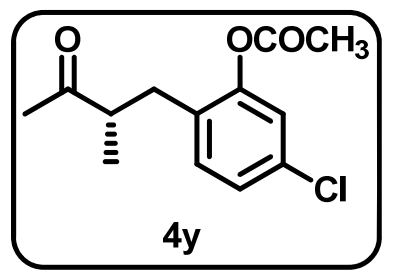

$\langle$ Chromatogram〉

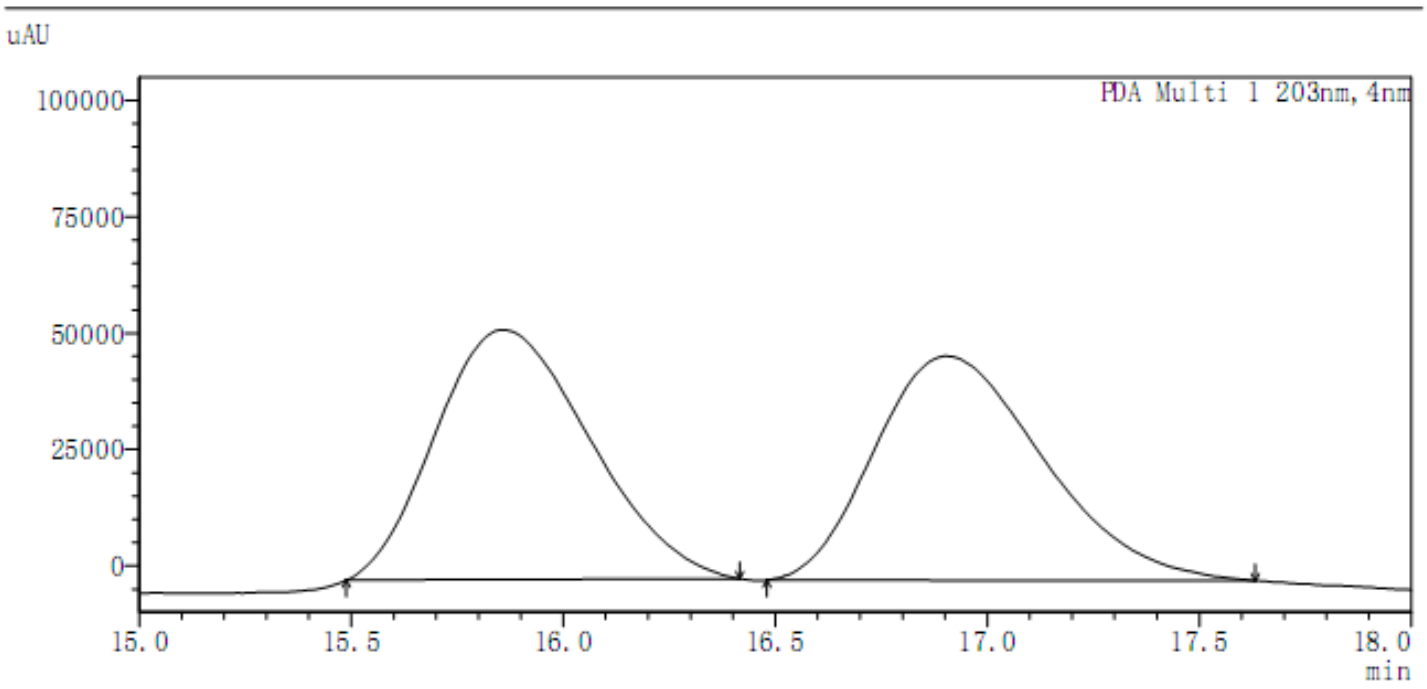

〈Peak Results〉

PDA Chl 203nm

\begin{tabular}{|c|c|c|c|c|}
\hline Index & Time/min & Height/mAU & Quantity/Area & Area \%/\% \\
\hline 1 & 15.859 & 53689 & 1358084 & 50.403 \\
\hline 2 & 16.904 & 48206 & 1336383 & 49.597 \\
\hline
\end{tabular}

$\langle$ Chromatogram〉

mAU

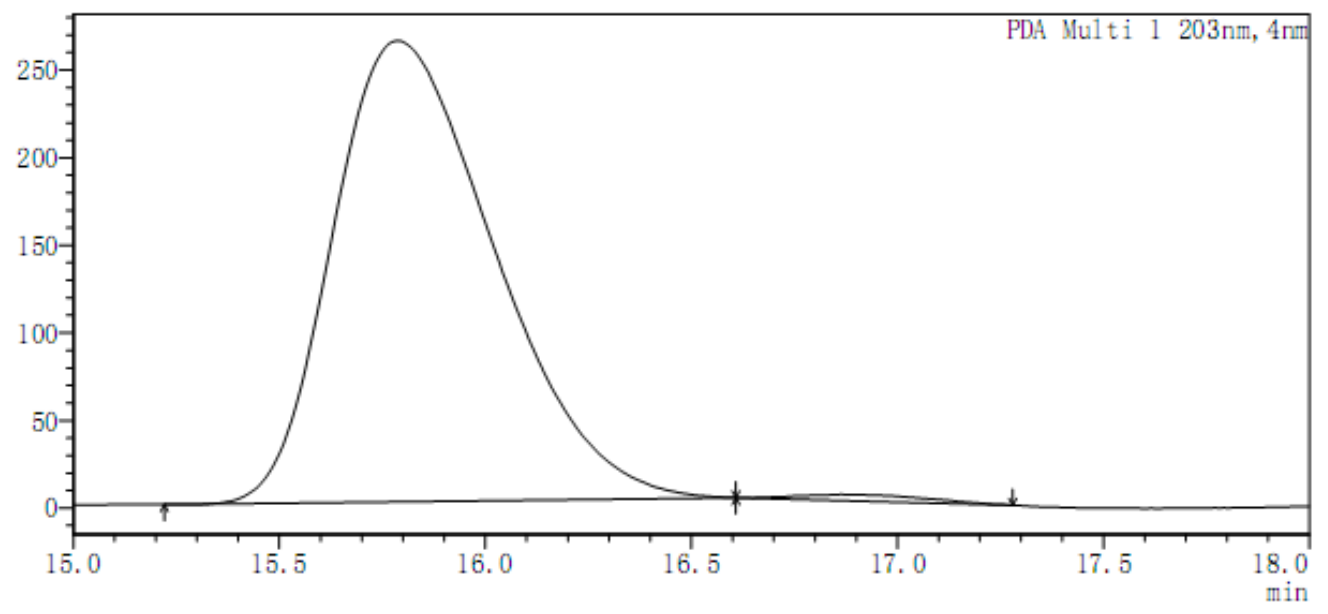

〈Peak Results〉

PDA Ch1 203nm

PDA Chl $203 \mathrm{~nm}$
\begin{tabular}{|c|c|c|c|c|}
\hline Index & Time/min & Height/mAU & Quantity/Area & Area \%/\% \\
\hline 1 & 15.788 & 263220 & 7188828 & 98.913 \\
\hline 2 & 16.865 & 3504 & 79038 & 1.087 \\
\hline
\end{tabular}




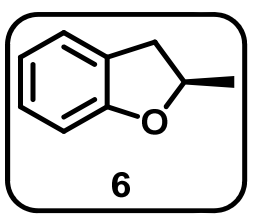

$\langle$ Chromatogram〉

mAU

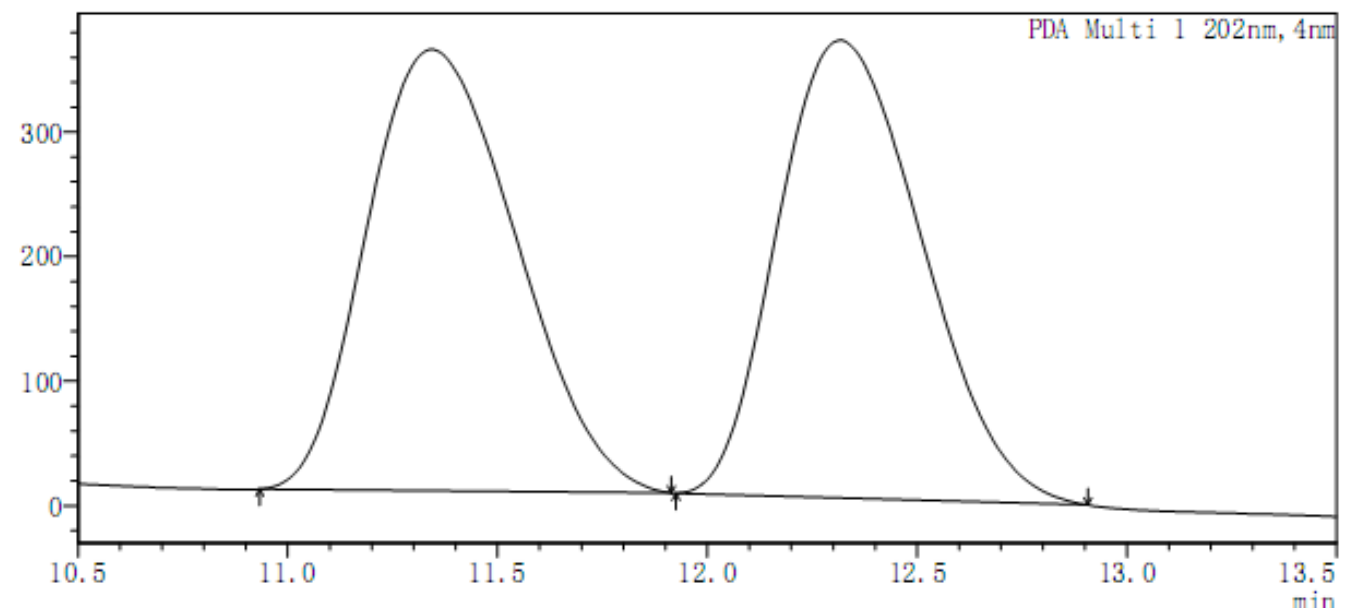

$\langle$ Peak Results>

PDA Ch1 202nm

\begin{tabular}{|c|c|c|c|c|}
\hline Index & Time/min & Height/mAU & Quantity/Area & Area \%/\% \\
\hline 1 & 11.343 & 354305 & 8772573 & 49.939 \\
\hline 2 & 12.317 & 367652 & 8793979 & 50.061 \\
\hline
\end{tabular}

〈Chromatogram〉

$\mathrm{mAU}$

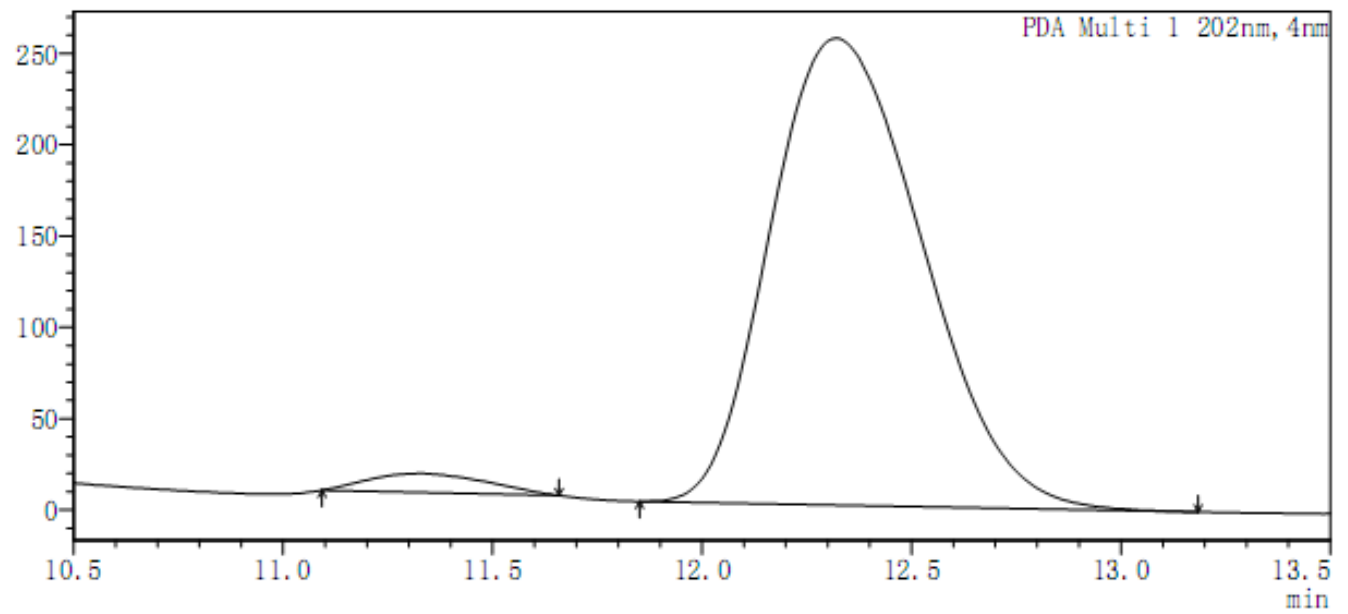

〈Peak Results〉

PDA Chl 202nm

\begin{tabular}{|c|c|c|c|c|}
\hline Index & Time/min & Height/mAU & Quantity/Area & Area \%/\% \\
\hline 1 & 11.325 & 10284 & 203090 & 3.048 \\
\hline 2 & 12.321 & 255812 & 6459836 & 96.952 \\
\hline
\end{tabular}

